\title{
INVESTIGATION OF FACTORS AFFECTING THE ADOPTION OF INFORMATION AND COMMUNICATION TECHNOLOGIES FOR COMMUNICATION OF RESEARCH OUTPUT IN RESEARCH INSTITUTIONS IN KENYA
}

By

Florence Nzisa Nzwilli Muinde

Principal supervisor: Prof. Gary Gorman

Associate supervisor: Dr. Chern Li Liew

A thesis submitted in fulfillment of the requirements for the degree of Doctor of Philosophy in Information Systems

School of Information Management

Victoria University of Wellington

Wellington, New Zealand 2009 


\section{DECLARATION}

I certify to the best of my knowledge and belief that the thesis is my own work and does not incorporate without acknowledgement any material previously submitted for a degree or diploma in any university or published or written by another person.

Wellington, July 2009

Signature:

Name: Florence N. N. Muinde 


\begin{abstract}
Using Rogers' (2003) and Hofstede’s (2001) technology diffusion theories as lenses, this exploratory and interpretive study was an endeavour to contribute to the understanding of ICT-enabled research communication by and for scholars and researchers working in Kenya. The main purpose of the study was to identify factors affecting ICT-enabled research communication by researchers in research institutions in specific fields within the natural and applied sciences in Kenya, which are viewed as key result areas in socioeconomic development.
\end{abstract}

Qualitative techniques were used to collect and analyze the data and present the findings. The researcher sought to identify, understand and explain key factors affecting ICTmediated scientific research communication with a view to coming up with an ICTadoption framework that would assist the Kenyan research community in more effectively adopting ICT-enabled research dissemination practices. This in turn should support Kenya's national development goals and contribute to the existing knowledge base and serve as a useful reference point in research communication debates and policy deliberations.

The findings revealed researchers' priority research communication need was reinforcement of capacity for strategic research through recognising and prioritising research communication in budgetary planning. Thus, the findings call for investment in scientific and technological research and its communication, which includes improving tools and infrastructure, especially ICT-enabled ones like Internet connectivity and other e-resources.

The findings affirmed the literature and extant theories guiding the study but also revealed information unique to the Kenyan context. Among emerging factors affecting adoption of ICT for scientific research communication were socio-cultural factors such as appreciation and perception of ICT; attitude of the scientific research community; demographic issues such as age/level of qualification, gender, poverty and literacy levels; communication networks and traditional cultural values such as orature, communalism and education culture. There were also institutional factors which included issues to do with ICT governance such as political and institutional leadership and culture; institutional framework; policy and strategy and legal and regulatory framework; and control over mass media communication channels. Moreover, 
inadequate institutional capacity for ICT-mediated research communication, lack of demand for MIS for research and teaching, lack of recognition and motivation for researchers were found to hinder ICT-mediated research communication.

Though ICT had the perceived attributes of relative advantages, compatibility, complexity, observability and reliability, there were relative disadvantages that discouraged adoption. These included the need for hard- \& software and virus upgrades; its susceptibility to environmental factors; dependence on other infrastructures that may be unavailable or unreliable; and possibilities for information overload and plagiarism. Other factors affecting ICT adoption that emerged outside the preliminary model included the nature of discipline/type of data; personal/individual institution's initiative; telephone wire thefts and lack of ICT research.

All these contextual perspectives informed the framework for adoption of ICT for scientific research communication by researchers and scholars in research institutions in Kenya.

Keywords: Information and Communication Technology (ICT); research communication in Africa; e-research; research institutions/Kenya; research communication needs/Kenya; technology diffusion; Rogers' Diffusion of Innovation; Hofstede's cultural dimensions perspectives 


\section{ACKNOWLEDGEMENT}

I wish to acknowledge the contribution of many organisations and individuals that made this research a success. First, I thank Commonwealth New Zealand for the scholarship that also took care of my family here. I also express my gratitude to the government of Kenya for study leave and great support during the $\mathrm{PhD}$ study.

I am indebted to the School of Information Management, right from the Head of School, the research coordinator, academic staff, down to the school administrators for support and enabling environment. Special thanks to Jean Grant and her team over the 3 and 1/4 years for their efficient support and being always there for the student.

I acknowledge my research institutions and participants in Kenya for their valuable insights which are the hallmark of the thesis.

I thank my two supervisors, Prof. Gary Gorman and Dr. Chern Li Liew, for their guidance and great enthusiasm in my work. I also acknowledge Dr. Deborah Laurs of Student Learning Support Service for her fine tooth for details in editing the work.

I would like to thank the following organizing committees for accepting papers based on this research for presentation and publishing:

1) The ICT for Africa Doctoral Consortium 18 December 2008, Yaoundé, Cameroon shared my research experience;

2) The ICT for Africa Conference 19-21 December 2008, Yaoundé, Cameroon: Muinde F. \& Gorman, G. (2008). "The Social-Cultural Environment in ICT-Enabled Research

\section{Communication in Kenya;"}

3) The IFLA World Library and Information Congress Milan, Italy, 23-27

August 2009: Muinde, F \& Gorman, G., (2009). "Barriers to Open Access to Scientific Information in Developing Countries: The Example of Kenya."

I owe special thanks to my husband, Dr. David Muinde Martin, for his great understanding and enduring support over the years. Moreover, I specially thank my four children - Maria Mbole, Mercy Ngina, Peter Matini and Onesmus Kaloki - for selflessly walking the journey with me and minding themselves as I indulged in the PhD.

I express my sincere appreciation to my brother, Onesmus Mutuku Nzwili, and his family for giving me a chance to pursue higher education and for his great 
encouragement throughout. I also acknowledge the support and prayers of other family members.

My PhD colleagues also deserve a special mention. Specifically, I would like to thank Lanthom, Vizaad, Silenge and my Commonwealth colleagues - Siamah Kaullychurn (Mauritius) and Abu Conteh (Sierra Leone) - for bracing it along with me. Further, I thank all my friends wherever they are for constant encouragement. I pay special tribute to Constancia Buchike (Mama Luwi) for being a great companion to me and children.

Above all, I owe it really to God's abundant grace and peace, which were indispensable ingredients for my $\mathrm{PhD}$ journey. 


\section{DEDICATION}

To my late mother, Maria Benedetta Mbole Nzwili, for her great belief in the liberating power of education, especially for the girl child:

Mama, you wondered why I couldn’t be the Dr. or Prof. mentioned in the class song in 1973 while in Grade 1. I have finally made it for you. 


\section{TABLE OF CONTENTS}

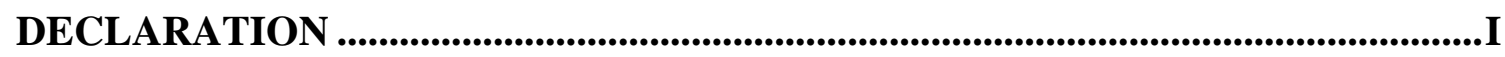

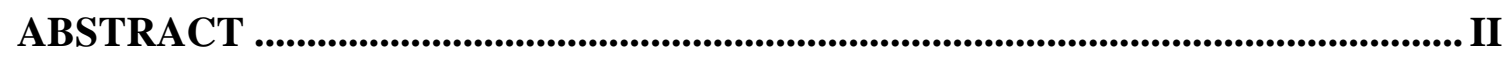

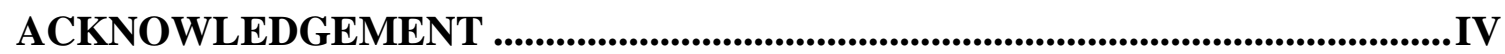

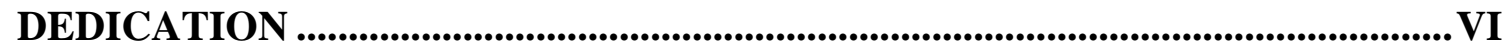

TABLE OF CONTENTS ................................................................................ VII

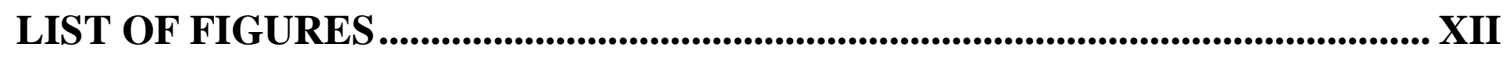

LIST OF TABLES .................................................................................................... XII

CHAPTER ONE:_INTRODUCTION................................................................- 1 -

1.0 BACKGROUND AND CONTEXT OF THE RESEARCH ............................. 1 -

1.1 STATEMENT OF THE PROBLEM ..................................................................... 4 -

1.2 RESEARCH OBJECTIVES..................................................................... 6 -

1.3 RESEARCH QUESTIONS............................................................................... 6 -

1.4 RESEARCH METHODOLOGY ............................................................... 7 -

1.5 SIGNIFICANCE OF THE STUDY ....................................................... 8 -

1.6 LIMITATIONS AND DELIMITATIONS OF THE STUDY .......................... 8 -

1.7 DEFINITION OF KEY TERMS............................................................... 9 -

1.8 ACRONYMS AND ABBREVIATIONS .............................................................- 10 -

1.9 ORGANISATION OF THE THESIS ..................................................... 11 -

CHAPTER TWO:_LITERATURE REVIEW .....................................................- 13 -

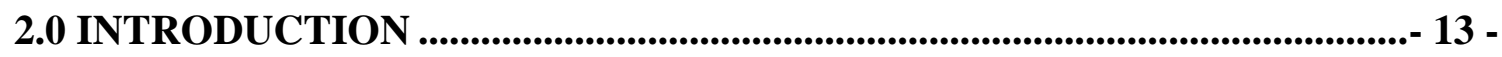

2.1 ICT AND DEVELOPMENT .....................................................................- 14 -

2.2 RESEARCH COMMUNICATION AND ITS IMPORTANCE .....................- 17 -

2.3 TRENDS IN RESEARCH COMMUNICATION ........................................... 21 -

2.3.1 Global trends in research communication ............................................................... 21 -

2.3.2 Research communication in Africa ............................................................................. 27 - 
2.3.3 Research Communication in Kenya ............................................................................. 32 -

2.4 ICT AND SCIENTIFIC RESEARCH COMMUNICATION .......................... 38 -

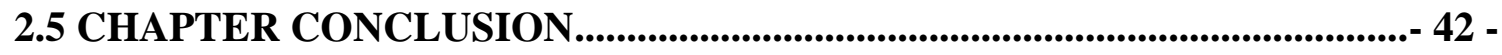

CHAPTER 3:_THEORETICAL FRAMEWORK................................................. 43 -

3.0 CHAPTER OVERVIEW ............................................................................. 43 -

3.1 RELEVANT TECHNOLOGY DIFFUSION \& ACCEPTANCE MODELS - 43 -

3.1.1 Rogers' Diffusion of Innovations theory (DOI) …..................................................... 49 -

3. 1.1.1 Variables determining the rate of adoption of innovations ................................... 51 -

3.1.1.2 Use of DOI in information studies research and justification for use in this study - 55

3.1.2 Hofstede's Cultural Dimensions model............................................................................ 58 -

3.1.2.1 Use of Hofstede's Cultural Dimensions model in the literature ............................. 63 -

3.1.2.2 Use of Hofstede's Cultural Dimensions model in information research ................ 64 -

3.1.2.3 Use of Hofstede's Cultural Dimensions theory in this study .................................. 67 -

3.2 THE RESEARCH FRAMEWORK ............................................................ 72 -

CHAPTER FOUR:_STUDY DESIGN AND METHODOLOGY ........................- 78 -

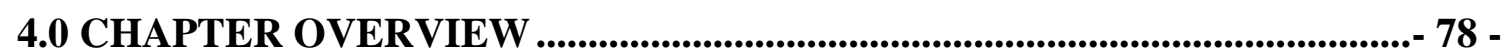

4.1 INTERPRETIVIST PARADIGM.................................................................... 78 -

4.2 QUALITATIVE RESEARCH DESIGN .................................................. 79 -

4.3 THE ROLE OF THE RESEARCHER.......................................................... 83 -

4.4 DATA GATHERING TECHNIQUES ........................................................ 85 -

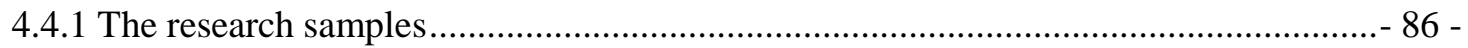

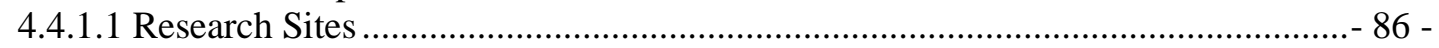

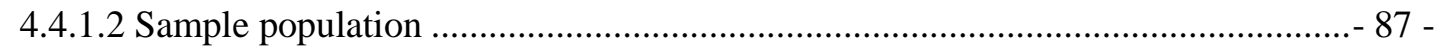

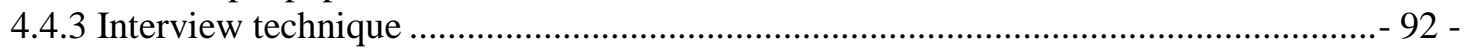

4.4.3.1 Interview procedure ……………………............................................................ 97 -

4.5 DATA ANALYSIS TOOLS AND METHODS.................................................- 98 -

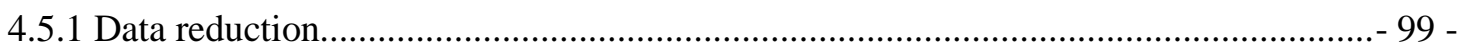

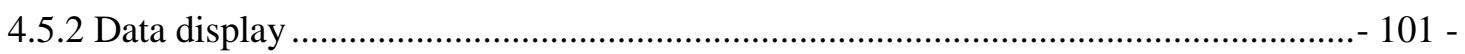

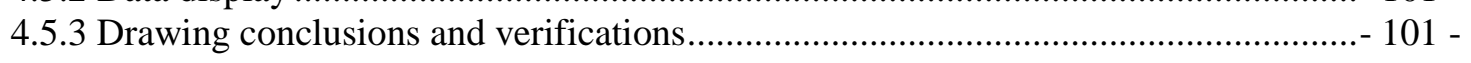

4.6 EVALUATING QUALITATIVE RESEARCH ............................................- 102 -

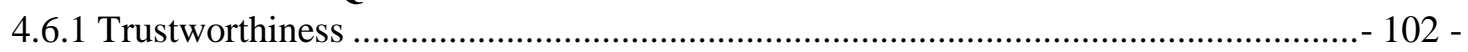

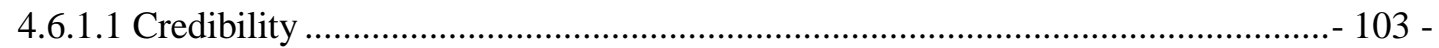

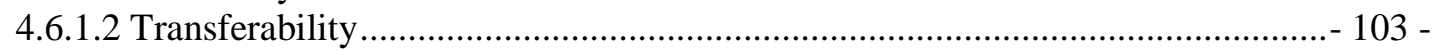

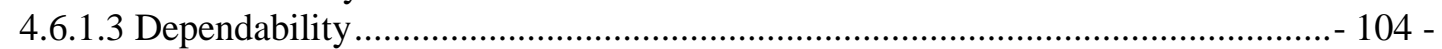

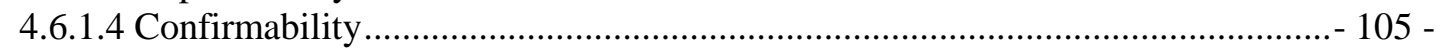

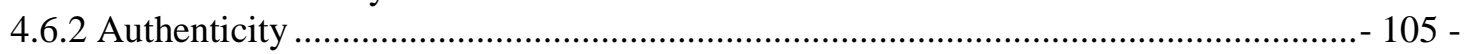

4.6.3 Klein and Myers' seven principles for interpretive research in IS ............................ 106 -

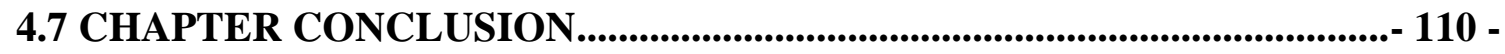


CHAPTER FIVE:REINFORCING CAPACITY FOR STRATEGIC RESEARCH COMMUNICATION

5.0 CHAPTER OVERVIEW

\subsection{PRIORITISING AND INVESTING IN SCIENTIFIC AND}

TECHNOLOGICAL RESEARCH COMMUNICATION

5.2 REINFORCING THE REQUIREMENT TO DO AND COMMUNICATE

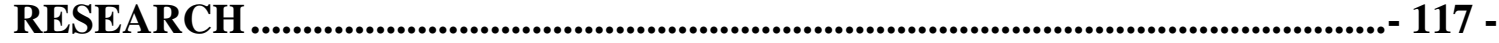

5.2.1 National politics and culture.................................................................................. 117 -

5.3 PROVIDING A MECHANISM FOR RESEARCH COMMUNICATION.- 141 -

5.4 BUILDING SKILLS CAPACITY FOR RESEARCH COMMUNICATION- 152

5.5 RECOGNISING AND MOTIVATING RESEARCHERS.

157

CHAPTER SIX:_THE SOCIO-CULTURAL ENVIRONMENT IN ICTMEDIATED RESEARCH COMMUNICATION IN KENYA ............................. 160 -

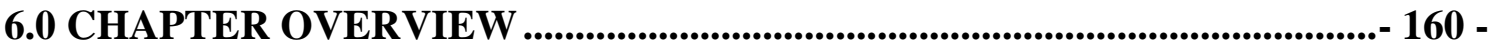

6.1 SOCIO-CULTURAL FACTORS ................................................................. 161 -

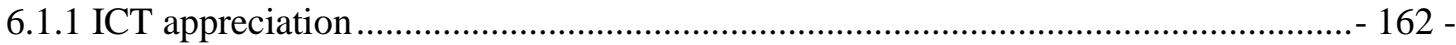

6.1.1.1 Lack of Understanding..................................................................................... 162 -

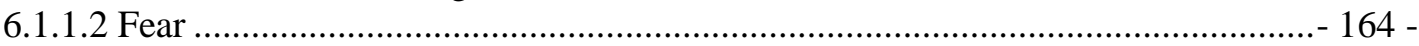

6.1.2 Perceptions about ICT potentiality and use ............................................................... 170 -

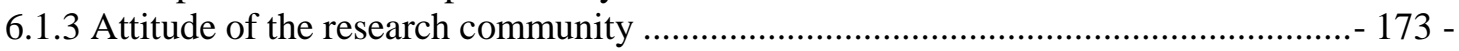

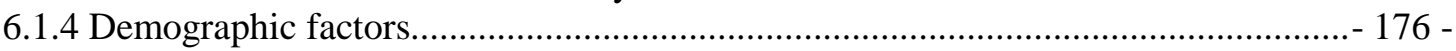

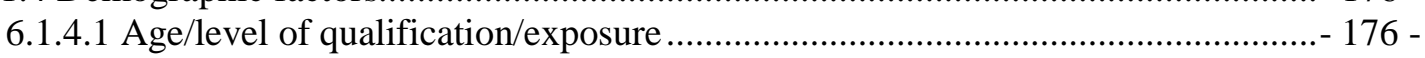

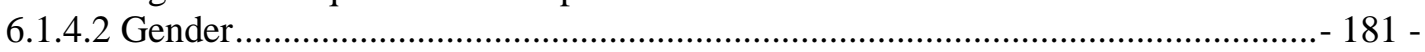

6.1.4.3 Poverty and low literacy levels .................................................................. 187 -

6.1.5 Lack of/need for connections/networks for ICT adoption........................................... 189 -

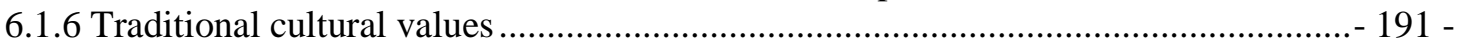

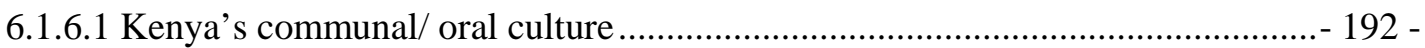

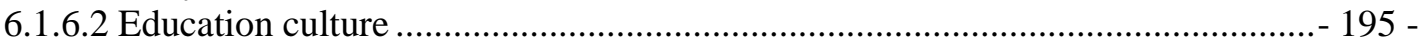

6.2 CHAPTER SUMMARY ................................................................................ 198 -

CHAPTER SEVEN:_THE INSTITUTIONAL ENVIRONMENT.......................- 201 -

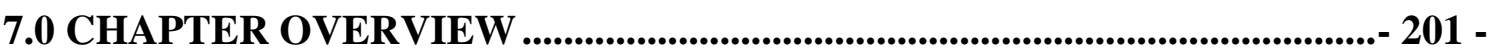

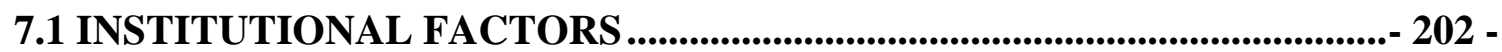

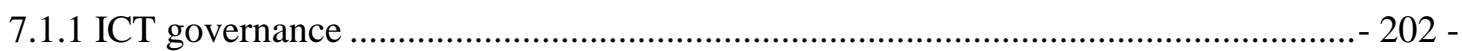

7.1.1.1 Political and institutional leadership and culture ............................................ 203 -

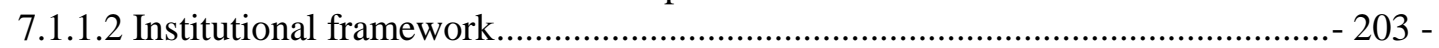

7.1.1.3 Policy, strategy, legislation and regulatory frameworks .................................... 209 -

7.1.1.4 Control over mass communication channels ........................................................ 213 -

7.1.2 Capacity for ICT-mediated research communication............................................... 215 -

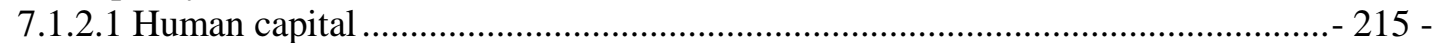

7.1.2.2 Lack of assessment of researchers' needs and institutional e-readiness ..............- 220 -

7.1.2.3 ICT tools and infrastructure ............................................................................ 221 - 
7.1.2.4 Appropriate content ......................................................................................... 226 -

7.1.2.5 Demand for research/teaching management information systems (MIS)............ 229 -

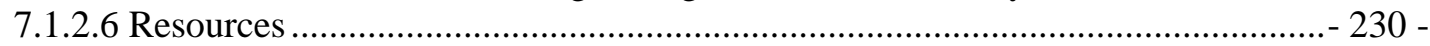

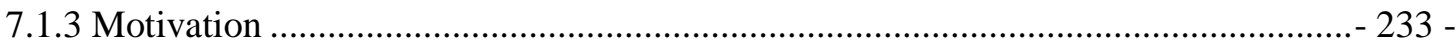

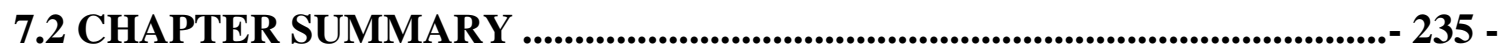

CHAPTER EIGHT:TECHNOLOGICAL AND OTHER FACTORS................- 239 -

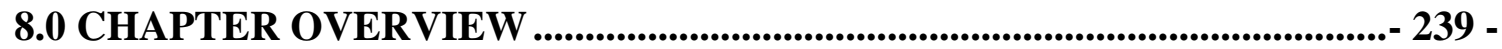

8.1 THE TECHNOLOGICAL DIMENSION ................................................ 239 -

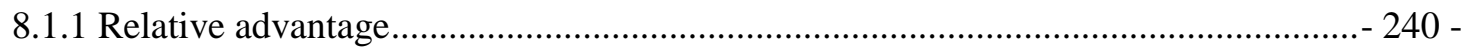

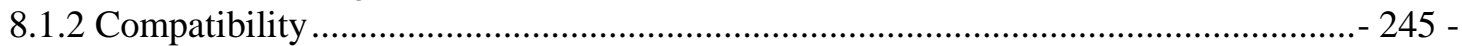

8.1.3 Complexity/ trialability /ease of use ........................................................................ 246 -

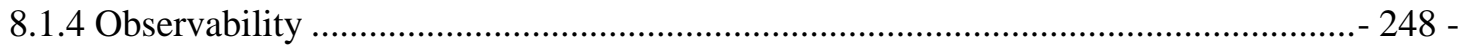

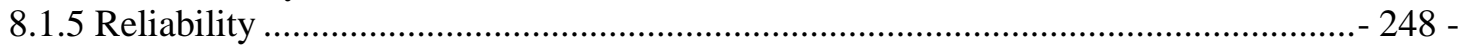

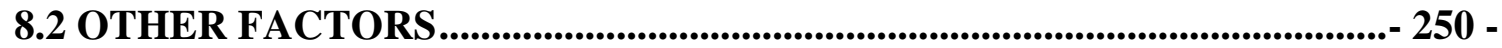

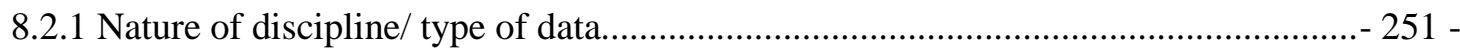

8.2.2 Personal/individual institutions' initiative................................................... 252 -

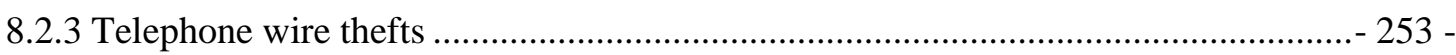

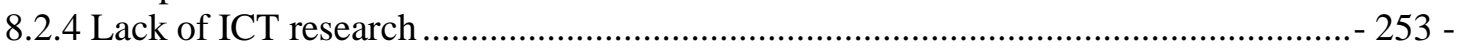

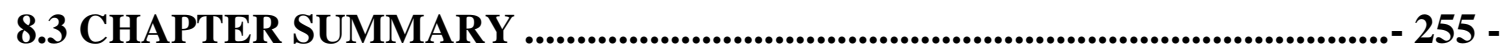

CHAPTER NINE:_A CONTEXTUAL FRAMEWORK, RESEARCH

CONCLUSIONS AND IMPLICATIONS .......................................................... 258 -

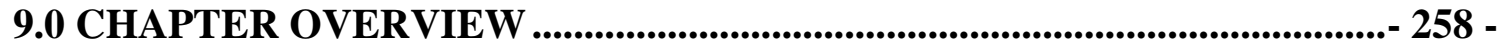

9.1 DATA ANALYSIS AND DISCUSSION OVERVIEW ................................... 258 -

9.2 A CONTEXTUAL FRAMEWORK FOR ICT-MEDIATED RESEARCH

COMMUNICATION ....................................................................................................- 263 -

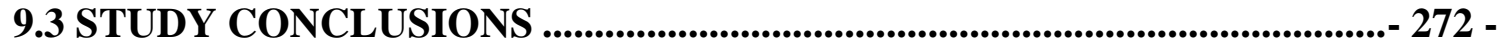

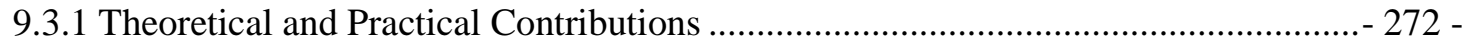

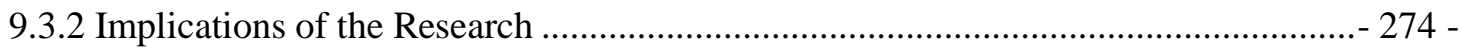

9.3.3 Recommendations for Future Research................................................................... 276 -

9.3.4 Concluding Remarks ................................................................................................ 277 -

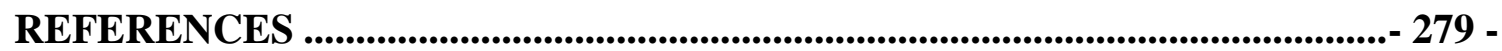

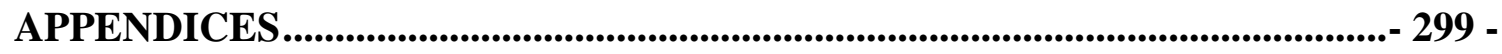

A-1: TIMELINE FOR THE PHD. STUDY …....................................................... 299 -

A-2: LETTER OF INTRODUCTION TO THE HEADS OF THE RESEARCH

INSTITUTIONS ................................................................................... 300 -

A-3: CURRENT SITUATION OF ICT INFRASTRUCTURE IN KENYA......- 301 - 
A-4: SUMMARY OF SELECTED EMPIRICAL STUDIES ON DIFFUSION OF IT-RELATED INNOVATIONS.............................................................................- 304 A-5: SUMMARY OF EMPIRICAL STUDIES ON THE CULTURAL FACTOR IN IT/ICT DIFFUSION .................................................................................................- 306 - 


\section{LIST OF FIGURES}

Figure 2.1: The four functions of scientific communication .................................... 19 -

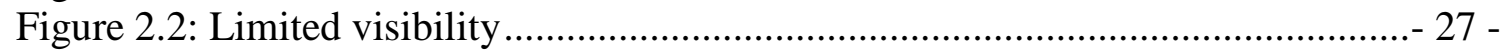

Figure 3.1: Variables determining the rate of adoption of innovations........................- 52 -

Figure 3.2 Proposed framework for investigating factors affecting diffusion and adoption

of ICT for research communication in Kenya ............................................................ 74 -

Figure 4.1: The recursive research process................................................................ 82 -

Figure 4.2: Components of data analysis: Interactive Model ...................................... 99 -

Figure 6.1: Revision of the socio-cultural dimensions .............................................. 199 -

Figure 7.1: Revision of the institutional dimensions .................................................. 237 -

Figure 8.1: Revision of technological dimensions ............................................... 256 -

Figure 8.2: Revision of other dimensions/factors................................................... 257 -

Figure 9.1: A contextual model for ICT-mediated scientific research communication in

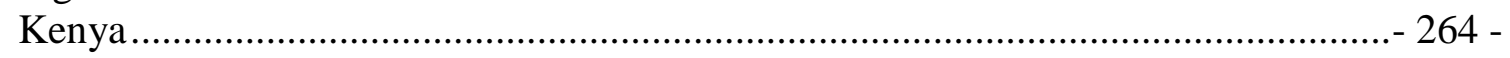

\section{LIST OF TABLES}

Table 2.1: Functions of Research Publication System ..............................................-20 -

Table 2.2: Changes in Academia on the Path to Cyberscience .................................... 26 -

Table 3.1: Models and Theories of Technology Acceptance ...................................... 48 -

Table 3.2: Hofstede's Dimension of Culture Scales.................................................... 61 -

Table 3.2: Hofstede's Dimension of Culture Scales.................................................... 61 -

Table 3.4: Interview Schedule .................................................................................... 77 -

Table 4.1: Sample Population................................................................................. 90 -

Table 4.2: Links between Research Questions, Data Collection Techniques and Data

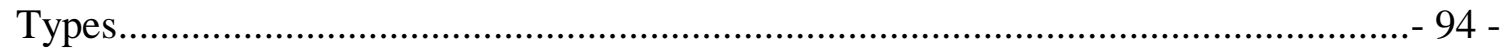

Table 6.1: Summary of Findings on Socio-Cultural Factors ..................................... 198 -

Table 8.1: Summary of Technological Factors.......................................................... 255 -

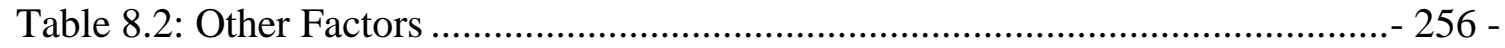

Table 9.1: Summary of Factors to Consider for a Contextual ICT-mediated Research

Communication Framework in Kenya................................................................... 262 -

Table A-3.1: Kenya’s Technology Achievement Index Status, 1995-2000 ...............- 302 -

Table A-3.2: Kenya’s ArCo Technology Index, 2004 ............................................ 303 - 


\section{CHAPTER ONE}

\section{INTRODUCTION}

\subsection{Background and Context of the Research}

In the global move towards knowledge-based economies powered by the ICT revolution, communication of research output to inform public debate and policy deliberations is a prerequisite in empowering communities to participate in the global knowledge economy. Application of Information Communication Technologies (ICT) has been seen as contributing to socio-economic, political, cultural and technological change in the development of the information society.

The ability of researchers in Kenya to adopt and use ICT in communicating research output as a means of contributing to policy formulation and other development-related activities was the motivation for this study. Using variables from Rogers' (1995, 2003) Diffusion of Innovation (DOI) theory and Hofstede’s (2001) Cultural Dimensions theory as initial parameters to guide the study, the ensuing research framework focused on the institutional, social, cultural and technological environment within which ICT adoption for research communication takes place.

Countries are now demanding the publication and communication of research outputs, a public good, as well as greater transparency, accountability and justification of the heavy investment in research projects and institutions. For example, the House of Commons Science and Technology Committee, Britain, in their scientific publishing report of 20 July 2004 recommended research outputs be made free for all (House of Commons Science and Technology Committee 2004; Willinsky 2006). The report required:

- universities should be supported in establishing open archives in which faculty could deposit copies of articles that they otherwise published in journals

- that funding agencies should make depositing articles in such e-print archives a condition of the agency's grant

- the government should look at ways of fostering open access journals, while having the Office of Fair Trading monitor the publishing industry.

Following this commitment by the government, the Research Councils UK (RCUK) issued a position statement regarding research outputs from publicly-funded research: 
- Ideas and knowledge derived from publicly-funded research are made available and accessible for public use, interrogation and scrutiny as widely, rapidly and effectively as practicable

- Effective mechanisms are in place to ensure that published research outputs are subject to rigorous quality assurance, through peer review

- The models and mechanisms for publication and access to research results are both efficient and cost-effective in the use of public funds

- The outputs from current and future research can be preserved and remain accessible not only for the next few years but for future generations.

The RCUK sees the new Internet-based models for the publication of research outputs and the underpinning data as likely to play an increasingly useful role in widening and improving access, which in turn supports the Research Councils' strategies for ensuring that the results of research are exploited more effectively for the benefit of the UK's society and economy.

Another case is the United States' House Appropriations Committee instruction to the National Institutes of Health (NIH) to arrive at a policy that would make NIH-funded research freely available through PubMed Central, a National Library of Medicine repository of open access biomedical research journals, within six months of the work's publication (NIH, 2003). This resulted in a policy release on 3 February 2005 urging NIH-funded investigators to submit an electronic version of the author's final manuscript upon acceptance for publication to PubMed Central, to be released to the public “as soon as possible (and within 12 months of the publisher's official date of final publication)” (NIH, 2003, n.p; Willinsky, 2006, p. 3). The policy has been reviewed twice since 2005 (NIH, 2008; NIH, 2009).

The UK and US cases indicate an acknowledgement by government that it is the public's right to have access to publicly funded research, and, therefore, they are justified to expect the same from the research community and government.

The international concern for increased accessibility to scientific research has also been addressed by international organisations. For example, in December 2003 a meeting of the World Summit of the Information Society (a UN initiative) adopted several recommendations relating to the publishing industry and produced a Declaration of Principles and a Plan of Action where the signatories declared: "We strive to promote universal access with equal opportunities for all to scientific knowledge and the creation 
and dissemination of scientific and technical information, including open access initiatives for scientific publishing” (World Summit on the Information Society 2003).. To ensure access to information and knowledge, their Plan of Action seeks to "encourage initiatives to facilitate access, including free and affordable access to open access journals and books, and open archives for scientific information” (World Summit on the Information Society, 2003). Similarly, the Organization for Economic Cooperation and Development (OECD) Committee for Scientific and Technological Policy agreed that "coordinated efforts at national and international levels are needed to broaden access to data from publicly funded research and contribute to the advancement of scientific research and innovation” (OECD, 2004).

The Association of African Universities (AAU) echoes the same in its strategic plan, which emphasises the central role of knowledge as a critical determinant in national development and international competitiveness, and demands that African higher education institutions play a more direct role in development than in the past. It asks African universities to improve the quality of teaching and research and the effectiveness of their contribution to policy, production, management and social construction, as well as their cost-effectiveness, as matters of increasing public concern (Association of African Universities, 2004). One way to achieve these goals may be by the adoption of ICT to the research communication process.

Kenya also recognises in many recent policy documents (Government of Kenya 2003; Government of Kenya 2003; Government of Kenya 2005; Government of Kenya 2005) and the Economic Recovery Strategy for Wealth and Employment Creation (ERSWEC) 2003-2007 (Government of Kenya 2004) and Vision 2030 (Government of Kenya 2007) that a major contribution from university education to national development is through the use of research outputs. The Government of Kenya sees research as a means of creating wealth and enhancing human development and, therefore, as vital in industrial transformation, economic growth and poverty reduction. However, despite the critical role research plays in national development, the utilisation of research has remained elusive due to:

- inadequate mechanisms for dissemination and utilisation of research findings

- $\quad$ absence of an up to date research databank, inventories and directories of what has been done or is being done

- ineffective coordination between the various actors 
- limited demand-driven and collaborative research between research institutions and the ultimate beneficiaries of research knowledge either in government or private sector/industries (Government of Kenya 2003; 2005).

One policy document that provides a policy framework for the university education investment programme stipulates that university education and training will need to be technologically informed, research supported, democratically managed and globally marketable. It requires each university to maintain a record of academic productivity, including publications, awards, funds from third parties, and patents and royalties, and also to collaborate with partner institutions, establishing internal evaluation mechanisms for academic programmes and management and to ensure publication of the results (Government of Kenya, 2005).

The success of the above policies on technologically-informed communication of research outputs hinges on the availability of ICT, especially the Internet, and its adoption to the research communication process. However, the inaccessibility and invisibility of Kenyan research output nationally and internationally may be an indicator that the adoption of ICT for research communication has been slow or negligible, and that there may be contextual issues underlying this situation. Further, there has never been a framework for adoption of ICT-enabled research communication specific to the Kenyan context, despite the global move towards ICT-enabled research communication. To date there has been no investigation into the barriers and enablers to ICT-mediated research communication in Kenya, or possible model frameworks for this process. A principal purpose of this study was to develop a contextual framework for ICT-enabled research dissemination for Kenya-based researchers in specific fields within the applied sciences. To be able to do this, the study identified the communication needs and priorities of researchers and the socio-cultural, institutional, technological and the intellectual factors affecting ICT-enabled research communication and dissemination by and for researchers in Kenya.

\subsection{Statement of the Problem}

Although Kenya is said to possess one of the largest scientific communities in Africa (Duque, Ynalvez, Sooryamoorthy, Mbatia, Dzorgbo, \& Shrum, 2005), the research output of researchers in Kenya has not been accessible or widely disseminated even with the advancement in ICT. Despite considerable discussion about how ICT can offer 
opportunities for improving research communication and bridging the digital divide in research, a recent comparative analysis of scientists in Ghana, Kenya and Kerala (India) on the productivity and collaboration paradoxes found that "African scientists make limited use of the Internet to reduce research problems” (Duque, et al. 2005, p.777). Yet there is apparent lack of discussion at present about the enablers and barriers of adoption of ICT for research communication among researchers in Africa generally.

Musa, Mbarika, and Meso (2005) attest to lack of research on ICT adoption and information systems in Sub-Saharan Africa (SSA) and recommend and justify the necessity of research in this area if SSA is to catch up with the rest of the world.

Newell, Swan and Galliers (2000) testify to the lack of research on processes surrounding the adoption of complex technologies like ICT: "Little research to date has focused on exploring these processes of knowledge unbundling, but the knowledgefocused perspective suggests that such research is vital to understanding increasingly interactive innovation processes” (p.255).

Duque et al. (2005) argue that "it is dangerous to speculate about the effects of the Internet on the production and dissemination of knowledge without close attention to local context (represented by country or region) and institutional context (represented by organisational type)” (p.776). Further, their research found that local contexts influence the adoption and use of ICT: "Productivity and collaboration paradoxes allude to the puzzles generated by contextual conditions that shape differential or negative impacts of new information and communication technologies” (p.778). They conclude by recommending that, "while one response to the productivity paradox is simply that not enough has been invested in ICTs, it seems crucial for students of science and technology to take a more critical view of collaboration and its constraints” (p.778).

Another study by Gebremichael and Jackson (2006) underscores the need to investigate why some user groups adopt, or fail to adopt, ICT "within the context of community norms” (p. 270). They recommend further research to determine how Sub-Saharan Africa ICT user groups interact within this ever-changing environment and advocate both qualitative and quantitative research to provide a user-centred framework for developing and building ICT in the region. 
The findings and recommendations of the studies discussed above point to the problem addressed in this research, which consisted of two elements. First, little is known about the actual research communication needs of researchers in Kenya or which of those needs have the highest priorities. Second, there is a lack of understanding of the factors that enable or hinder ICT-enabled research communication by researchers in Kenya. By understanding both elements of the problem, it should be possible for appropriate agencies in Kenya (research institutions and relevant government ministries) to plan and implement infrastructures for improving the level of adoption and use of ICT for improved research communication. This in turn should support Kenya's national development goals through informed policy and decision making processes.

\subsection{Research Objectives}

The study sought to explore the barriers and enablers (organisational, social, cultural, political and technological) associated with ICT adoption for the communication/dissemination of research output by researchers working in selected research institutions in Kenya, with a special focus on some of Kenya's key economic recovery areas: agriculture, biology/biotechnology, environmental and health sciences. Specifically, the study sought to meet the following objectives:

1. To develop a preliminary model of factors that affect ICT-enabled research communication by Kenyan researchers in agriculture, biology/biotechnology, environmental science and health sciences

2. To identify the communication needs and establish priorities of communication needs for Kenyan researchers in agriculture, biology/biotechnology, environmental science and health sciences

3. To identify and understand enablers for and barriers to ICT-enabled research communication by Kenyan researchers in agriculture, biology/biotechnology, environmental science and health sciences.

\subsection{Research Questions}

This study addressed the following research questions:

1. What are the research communication needs of researchers in Kenya in agriculture, biology/biotechnology, environmental science and health sciences from the perspective of key research participants? 
2. What are the main priorities for research communication by researchers in Kenya in agriculture, biology/biotechnology, environmental science and health sciences?

3. What are the contextual factors that affect ICT-enabled research communication by researchers in Kenya in agriculture, biology/biotechnology, environmental science and health sciences?

4. How do these factors affect ICT-enabled research communication by researchers in Kenya in agriculture, biology/biotechnology, environmental science and health sciences?

\subsection{Research Methodology}

The study adopted the interpretivist epistemological position, which stresses the understanding of the social world through an examination of the interpretation of that world by its participants (Bryman, 2004; Creswell, 2003; Denzin \& Lincoln, 2005). Interpretivist paradigm posits reality as a holistic and constantly shifting emergent property of individuals' creation, thereby providing much needed insights into information-seeking experiences (Bryman, 2004). This approach was chosen because the researcher's goal centred on understanding, rather than on predicting, what the various stakeholders in the research study believed, what the researchers considered as the most important research communication needs and priorities and how they felt and interpreted events pertaining to diffusion, adoption and use of ICT for research communication in Kenya. This served as a first step towards developing a framework for utilising modern ICT by researchers in Kenya to improve accessibility and visibility of Kenyan research output in both the local and the global knowledge economy.

Qualitative research methods were used to collect data. These entailed document analysis and individual face-to- face interviews which were used to collect data from researchers, research institution administrators, representatives from relevant government Ministries/departments, librarians and journal editors. These groups of participants were chosen because of their central role in the research communication process. The researchers, for example, undertake the research that produces the content that editors edit in preparation for dissemination. The administrators and government representatives formulate and implement policies and make important decisions on budget allocations that affect the research process and introduction of any new 
innovation. Research librarians enable researchers in their efforts to create content and at the same time serve to disseminate the research results to those who seek to use them.

\subsection{Significance of the Study}

On the practical level, the study made explicit the enablers and barriers to the adoption of ICT as a first step towards developing a contextual model framework for implementing an ICT-enabled research communication system for researchers in Kenya. Second, the study analysed the views and suggestions of researchers in Kenya in terms of ICT adoption for research communication. This should inform future strategies for ICT adoption and the development of contextual frameworks for assisting in exploiting modern ICT to improve visibility and accessibility of Kenyan research output, thereby facilitating greater participation in the global knowledge economy. Furthermore, the study should benefit researchers in Kenya and elsewhere in Africa who may be interested in knowing the potential of ICT-mediated research and also funding agencies interested in capacity-building for research communication.

Moreover, to date no qualitative study has been undertaken in research institutions in Kenya to explore enablers and barriers to the possibilities of enhancing research communication through using ICT. The few studies and projects on ICT that have been done are fragmented, often unsystematic and in most cases not theoretically informed (Adeya \& Oyelaran-Oyeyinka, 2002; Ochuodho \& Matunga, 2005; Gebremichael \& Jackson, 2006). They concentrated on information infrastructure issues, rather than processes like the adoption of ICT for research communication as a starting point for enhanced participation, visibility and accessibility of Kenyan research in the local and global knowledge arena. This study was, therefore, timely for advancing Kenyan scholarship in that it assisted in building a contextual framework for ICT adoption as well as identifying strategies for dealing with issues of research communication.

\subsection{Limitations and Delimitations of the Study}

The study was interpretive in nature and used qualitative methods to explore factors that affected the adoption of ICT for the communication of research output among researchers in selected research institutions in Kenya. The interpretive stance made the study subjective, thereby limiting its generalisability. The study addressed research communication as a process and not the publishing side, other than gathering information on the researchers' perceptions on e-publishing. Purposive sampling was 
used to select research sites and participants; thus further decreasing the generalisability of the findings of the study.

Another limitation associated with the interpretive qualitative design is that the results may be subject to other interpretations (Bryman, 2004). However, this study should be viewed as an exploratory enquiry into factors affecting the adoption of ICT for communication of research output by researchers in Kenya in specific disciplines, and makes no claims beyond this, with a view to coming up with a contextual framework of ICT-enabled research communication that is specific to the Kenyan context.

The in-depth interview that was the main data collection technique was also a limitation, with some researchers unwilling to be engaged for 1 to 1.5 hours owing to their busy schedule. The researcher alerted the interviewees in advance to the time required for an interview and interviewed only those willing to be engaged for that long.

Because of the limited time for field research that was allowed by the researcher's scholarship (6 months), the study focused only on the research communities in two Kenyan universities, and three national and three international research institutions based in Kenya (see table 4.1 in chapter 4). These institutions were chosen because of the relevance of their research interests to the discipline focus of the topic under investigation.

In this study, ICT designates the application of Information and Communication Technologies and services to facilitate acquisition, use and dissemination of research output through formal and informal channels. The researcher specifically investigated the factors affecting the adoption of the computer-mediated information and communication technologies, the Internet in particular, for communication of research output by researchers in research institutions in Kenya.

\subsection{Definition of Key Terms}

ICT - This refers to the Information and Communications Technologies, that is, technologies that are used to handle information and aid communication. In this study, the researcher specifically investigated the computer-mediated communication technologies, the Internet in particular. 
ICT-enabled research communication - designates the application of Information and Communication Technologies and services to facilitate acquisition, use and dissemination of research output through formal and informal channels.

Research - work done by scholars/researchers for promotion, teaching, professional development, or social good (Boettcher, 2006).

Research institutions - bodies or organisations mandated to undertake methodical investigations on areas of socio-economic, cultural and political concern of a country or any other entity. In this study, research institutions include universities and any other designated organisation involved in applied sciences and technology research, specifically in agriculture, biology/biotechnology, environmental science and health sciences.

\subsection{Acronyms and Abbreviations}

AAU - Association of African Universities

AJOL - African Journals Online

ARL - Association of Research Libraries

AVU - African Virtual University

DOI - Diffusion of Innovations

EALB - East African Literature Bureau

EAPH - East African Publishing House

ECA - Economic Commission for Africa

ERSWEC - Economic Recovery Strategy for Wealth \& Employment Creation

HINARI - Health InterNetwork Access to Research Initiative

ICIPE - International Centre for Insect physiology and Ecology

ICT - Information and Communication Technologies

INASP/PERI - International Network for the Availability of Scientific Publications/ Programme for the Enhancement of Research Information

KENET - Kenya Education Network 
KESSP - Kenya Education Sector Support Programme

MOEST - Ministry of Education, Science \& Technology

NEPAD - New Partnerships in Development

NIH - National Institutes of Health

UNCSTD - United Nations Commission on Science and Technology for Sustainable

Development

UNECA - United Nations Economic Commission for Africa.

\subsection{Organisation of the Thesis}

The thesis is organised into nine chapters. Chapter 1 sets the background and context of the research study, states the research problem, objectives, research questions, a summary of the methodology, significance of the study, limitations and delimitations of the study and definition of key terms and acronyms.

Chapter 2 reviews the literature on key concepts underlying the study with a view to establishing gaps in knowledge that necessitate the undertaking of the research, while chapter 3 discusses and further reviews literature for theoretical perspectives to guide the research study.

Chapter 4 highlights the research design and methodology used in collecting and analysing data to address the research objectives and questions.

Chapters 5- 8 present the findings of the research. Chapter 5 addresses the research communication needs and priorities of researchers in the applied sciences in Kenya emanating from their broad socio-cultural, political and institutional environments.

Chapters 6 and 7 respectively present findings related to factors of the socio-cultural and institutional environments that affected ICT-mediated research communication.

Chapter 8 addresses factors of the technological environment and other factors affecting adoption of ICT such as the nature of discipline/type of data; personal/individual institution's initiative; telephone wire thefts and lack of ICT research.

Chapter 9 briefly reviews the major findings in relation to the research objective and questions and briefly comments on the points of convergence and divulgence from the 
preliminary theoretical model presented in chapter 3. This is followed by presentation of the revised framework and research conclusions. 


\section{CHAPTER TWO}

\section{LITERATURE REVIEW}

\subsection{Introduction}

This chapter reviews literature on the key concepts underlying the study with a view to establishing gaps in the knowledge that necessitate the undertaking of the research. The review includes sections on ICT and development; Research communication and functions; Global trends in research communication; Research communication in Africa; Research communication in Kenya; ICT and research communication; relevant technology diffusion models in the literature; Models to be used in the study and the research framework based on relevant models and the literature.

This study is premised on the concept that an understanding of the organisational, social, cultural, economic and technological factors that affect adoption of ICT for research communication is a precursor of an advanced infrastructure on which innovative scholarly research and education environments can be built. It is important to address these dimensions because as Matsuura (2004) explains, while ICT have greatly facilitated the movement and handling of data, the process of generating and validating information and knowledge remains essentially one of human creativity. Longino (2002) talks of knowledge as having a social dimension, which serves to validate it. Hence, she stresses the need to pay more attention than ever before to the social dimensions that arise in the day-to-day conduct of scientific research work that may affect its circulation, an idea supported by Willinsky (2006), who calls for open access to research as a public good.

Willinsky (2006) laments the unavailability of research information despite the promise of increased access to information facilitated by ICT, arguing that, "the growth of the knowledge economy, which might otherwise have been thought to herald the university's ship coming in, has produced a 'serials crisis' that threatens the basic access principle otherwise critical to production of research and scholarship” (p.18). The Association of Research Libraries (2000) attests to the scarcity of research information occasioned by the rising cost of scholarly publishing. Willinsky (2006) says "to find new ways of increasing access is to extend an invitation and to acknowledge a right, for 
scholarship exists only as it is shared and is circulated, only as it is open to new and diverging voices” (p.32).

Nevertheless, research publishing and communication call for an efficient and effective communication system. ICT has been viewed as a harbinger to that. Atkins et al. (2003) talk of the need for an advanced "cyberinfrastructure ${ }^{1}$ layer of enabling hardware, algorithms, software, communications, institutions, and personnel” that provides "an effective and efficient platform for the empowerment of specific communities of researchers to innovate and eventually revolutionalize what they do, how they do it, and who participates” (p. 5). He says cyberinfrastructure is required for a knowledge economy just in the same way as infrastructure is required for an industrial economy. The same view is upheld by others Van de Sompel, Payette, Erickson, Lagoze, \& Warner, 2004; Warner, 2005). However, there is need to find out the social-economic, cultural, technological and institutional factors that affect the adoption and use of ICT in the research communication process if we are to come up with suitable and sustainable context specific electronic communication frameworks (Borgman, 2007; Kling, 1999; Kling \& Callahan, 2002; Zhang, 1998). This study is a response to the lack of a discussion on contextual factors affecting adoption of ICT especially for communication of research output in a developing country context, Kenya.

\subsection{ICT and Development}

Today's discussions of knowledge societies emphasise the need to harness ICT for development by enabling their use for empowering the poor and for scientific and technical capacity building that is consistent with development goals (Mansell \& Wehn, 1998; World Bank, 1998/1999). Pehrson (2005) sees universal access to ICT as an important part of the efforts to improve the living conditions in the world and, consequently, part of the United Nations' Millennium Development Goals and the roadmap to reach them. However, there is need for context specific ICT adoption frameworks informed by the socio-economic, political, cultural, institutional and technological backgrounds for enhanced adoption. Matsuura (2004) holds that national policies, supported by international frameworks, can be a tool to facilitate ICT-enabled access to information.

\footnotetext{
${ }^{1}$ Highest-performing technology based upon distributed computer, information and communication technology and its use in the most leading-edge research
} 
From the invention of the telegraph to the introduction of the Internet, the advancements in ICT have, over the years, gone hand in hand with modernisation and development (Dufborg, 2005). The former UN Secretary General, Kofi Annan, in his remarks in the first meeting of the United Nations Working Group on Informatics 1997, said that “Communications and Information Technology have enormous potential, especially for developing countries, and in furthering sustainable development” (Mansell \& Wehn, 1998, p.6). The World Bank argues that "knowledge for development” are crucial for the future prospects of developing countries, and it is increasingly important for these countries to be able to use and contribute to the growing stock of global knowledge (World Bank, 1998/1999).

The United Nations Commission on Science and Technology for Sustainable Development (UNCSTD) Working Group observed that the coming decades will not see the eradication of the gap between the rich and the poor unless governments and other stakeholders design and implement effective ICT strategies to transform data and information into useful knowledge that is consistent with development priorities (Mansell \&Wehn, 1998). This is why this study focuses on investigating the sociocultural, institutional and technological factors affecting the adoption of ICT for research communication by researchers in research institutions in Kenya, as a means of developing a contextual model for ICT-enabled research communication in Kenya. This is important because "a communication infrastructure is as essential for the development of society as transport, power, water and sanitation infrastructures” (Pehrson, 2005, p.5). For it to be successful, it must be informed by the socio-economic, cultural, institutional and technological background of the specific adopter community.

The African continent is lagging behind in the global information and technology revolution. Beyond the deficiencies in national ICT provision, higher education institutions that should be at the forefront of Africa's participation in the ICT revolution are, in most instances, ill-prepared to play such a leadership role because of the inadequate development of their own information infrastructure. Africa's research institutions are thus poorly positioned - when compared with their counterparts in Europe, North America and other developing regions, such as Asia - to take advantage of the global information economy and knowledge systems, and pioneer ICT's expansion in their countries. The development and application of ICT in African higher education institutions, therefore, becomes will be crucial and urgent if the continent is to 
reduce the knowledge, technological, and economic gaps between itself and the rest of the world ( Association of African Universities, 2004; Juma, 2002, 2008a, 2008b).

Development can be seen as an increase of knowledge and skills and creative potentials that can be applied to improve the quality of life. Knowledge is a critically important commodity in any society for it contributes to economic growth, technological development, cultural transmission and advancement, and political communication (Altbach, 1982). Well-informed, knowledgeable and innovative citizens are causes for human-centred development. Information technology facilitates the flow of knowledge in modern society. However, research shows that low levels of knowledge and inadequate innovative skills at lower, middle and higher levels have contributed to the continuous failures in African countries in all spheres (UNECA, 1996).

While Africa remains at a disadvantage in terms of both the production and distribution of knowledge, it is nevertheless affected by the worldwide knowledge revolution via modern ICT. Scholarly and scientific journals, which are one of the traditional knowledge distribution channels, have been affected by the information revolution in that on-line journals and databases are transforming knowledge distribution (Altbach, 1998). In today's knowledge economy, the ability to use and adapt technology is a critical factor and disinformation about Africa may be the most lasting wound inflicted on the continent by new patterns of dependency (Castells, 2000; Dahms, 2000). The central role of knowledge as a critical determinant of development and international competitiveness underscores the need for African universities and research institutions to exploit the benefits of ICT to improve the quality of teaching and research and the effectiveness of their contribution to policy, production, management and social construction, as well as their cost-effectiveness to facilitate the desired socio-economicpolitical change (Association of African Universities, 2004). This first of all calls for a context specific understanding of the enablers and barriers to diffusion, adoption and use of ICT in the particular environments.

Kenya recognises the crucial role of its research institutions in national development and sees adoption and use of ICT in the research communication process management and dissemination as the roadmap to the global knowledge economy (Government of Kenya 2003a, 2003b; 2005a, 2005b). It is in recognition of this that this study investigates the organisational, socio-cultural and technological factors that affect adoption of ICT to 
research communication among researchers in Kenya with a view to developing a contextual framework for adopting new ICT in research communication that can guide effective and efficient production, access, dissemination and use of research findings in solving developmental problems.

\subsection{Research Communication and its Importance}

Among the various types of research activities, research communication holds a central place and is linked with production and distribution of knowledge. Scholarly discourse and cooperation would be impossible without communication. Publications are products of research communication. Generally, the research community is a network of communicating people with different specialisations, and science and research is communication (Nentwich, 2003). Willinsky (2006) argues “a commitment to the value and quality of research carries with it a responsibility to extend the circulation of such work as far as possible and ideally to all who are interested in it and all who might profit by it” (p. xii). He explains that making research freely available is not only about human rights and greater circulation of knowledge but also increasing research impact.

Research communication serves to boost strategic research - it contributes to the growth of knowledge by improving the effectiveness and efficiency of research. Secondly, it contributes to proper knowledge management in that it is geared towards supporting scholarly research in evaluating results, institutions and researchers. In addition, research communication facilitates both the generation of relevant research problems (raising the right questions) and the solution to these problems (giving the right answers), which relate to the issues of use, availability and retrievability of information (Roosendaal \& Geurts, 1997).

Communication of research results serves to add new observations and ideas to what is already known; thus, creating a higher level of knowledge. Isaac Newton illustrated this metaphorically when he proclaimed, "If I have seen further it is by standing on the shoulders of giants” (Meadows, 1998, p.8). This metaphor connotes two implications about communication: (1) that the process of accumulation involves providing information about one's own work to others and, in return, receiving information from them; and (2) information must be made available in a durable, readily accessible form since the accumulation process stretches over time (Meadows, 1998). 
One of the conditions for scientific communication is that it should provide effective and efficient ways of filtering new research results that allow proper "aging" of research models and theories (Roosendaal \& Geurts, 1997). Thus certification should be subject to the conditions of strategic research for obvious reasons of consistency.

Figure 2.1 below represents the four main functions of scientific communication: registration, awareness, certification and archiving. It shows the overall communication process as it is embedded within the research process. The vertical axis describes registration and awareness, which can both be seen as different aspects of scientific observation; the horizontal axis describes certification and archiving, which can be seen as different aspects of scientific judgment. The registration function is both a concrete and objective one; the awareness function is abstract and subjective; the certification function is concrete and subjective; and the archive function is abstract and objective. There is a half plane of two objective functions (registration \& archiving) and a half plane of two subjective functions (awareness \& certification). The subjective communication functions are the ones that are internal to the research process itself, whereas the objective functions are external to the research process and can easily be outsourced within the market to the product space, viz. the publisher and the library. However, it should be noted that scientific communication results above all form the interactions or transactions between the functions, which include the transfer of content (primarily the author-reader interface) and the transfer of (consolidated) knowledge (primarily the subjective-objective interface). The latter is in particular important for strategic research as it largely determines its degree of applicability (Roosendaal \& Geurts, 1997).

From figure 2.1 below, the archive function serves as a main transaction function or sluice between author and reader. This raises the immediate issue of integrating informal communication into the platform of such an archive leading to an integration of formal and informal communication, which means formalising informal communication into one and the same platform and management system. A result of these developments is that the now distinct roles of publishers and libraries will be merged to become nodes in the overall management of scientific communication (Roosendaal \& Geurts, 1997). Moreover, in the paper-based era the published article in a refereed journal is archived in an ad hoc fashion as libraries shelf it across the world (Van de Sompel, et al. 2004); thus limiting access. This necessitates the need to explore possibilities of alternative models 
of research communication, especially ICT-enabled ones, which could enhance accessibility of research outputs. This study was an attempt to develop a contextual framework for adoption of ICT for research communication for enhanced scientific research accessibility and visibility by researchers in Kenya.

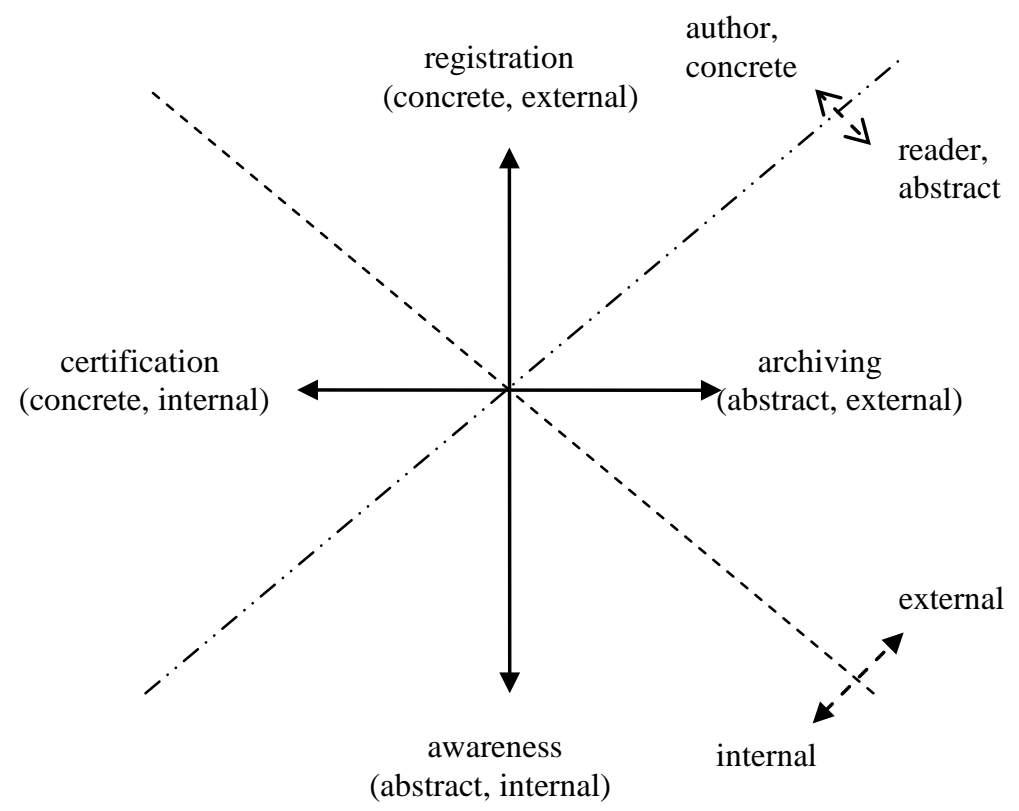

Figure 2.1: The four functions of scientific communication (Roosendaal \& Geurts, 1997).

There is a close link between the four functions of communication represented in figure 2.1 above and the object of this research. The research investigated the socio-cultural, institutional and technological factors that affect the adoption of ICT for research communication, which in essence are the registration, awareness, certification and archiving functions.

As summarised in table 2.1 below, other scholars share and add to Kircz and Rosendaal's (1996) views on the functions of research publication system (Franks, 1993; Guedon, 1994; Kircz \& Roosendaal, 1996; Morton, 1997; Kling \& McKim, 1999; Nentwich, 2003; Kling, 2004; Van de Sompel, et al., 2004; Thompson, 2005; Warner, 2005; Borgman, 2007). The publication system is the nucleus of formal communication among researchers (Nentwich, 2003). 
Table 2.1: Functions of research publication system

\begin{tabular}{|c|c|}
\hline Source & Functions of research publishing system \\
\hline Franks (1993) & $\begin{array}{ll} & \text { Certification } \\
\text { - } & \text { Distribution } \\
\text { - } & \text { Marketing } \\
\text { - } & \text { Archiving }\end{array}$ \\
\hline Guedon (1994) & $\begin{array}{ll} & \text { Legitimisation \& authority } \\
\text { - } & \text { Communication \& diffusion } \\
\text { - } & \text { Archiving \& memory }\end{array}$ \\
\hline Kircz \& Roosendaal (1996) & $\begin{array}{ll}\text { - } & \text { Certification } \\
\text { - } & \text { Registration, i.e. ownership protection } \\
\text { - } & \text { Platform for communication } \\
\text { - } & \text { Archiving }\end{array}$ \\
\hline Morton (1997) & $\begin{array}{ll} & \text { Serial communication } \\
\text { - } & \text { Public disclosure } \\
\text { - } & \text { Feedback from informed readers to authors or editors } \\
\text { - } & \text { Preservation of data }\end{array}$ \\
\hline Roosendaal \& Geurts (1997) & $\begin{array}{ll} & \text { Certification } \\
\text { - } & \text { Registration } \\
\text { - } & \text { Awareness } \\
\text { - } & \text { Archiving }\end{array}$ \\
\hline $\begin{array}{l}\text { Kling (2004); Kling \& } \\
\text { McKim (1999) }\end{array}$ & $\begin{array}{ll}\text { - } & \text { Trustworthiness } \\
\text { - } & \text { Publicity } \\
\text { - } & \text { Accessibility }\end{array}$ \\
\hline Crow, R. (2002) & $\begin{array}{ll}\text { - } & \text { Registration } \\
\text { - } & \text { Certification } \\
\text { - } & \text { Awareness } \\
\text { - } & \text { Archiving }\end{array}$ \\
\hline $\begin{array}{l}\text { Van de Sompel, et al. } \\
\text { (2004) }\end{array}$ & $\begin{array}{ll} & \text { Certification } \\
\text { - } & \text { Registration } \\
\text { - } & \text { Awareness } \\
\text { - } & \text { Archiving } \\
\text { Rewarding }\end{array}$ \\
\hline Nentwich (2003) & $\begin{array}{ll}\text { - } & \text { Certification } \\
\text { - } & \text { Registration } \\
\text { - } & \text { Diffusion } \\
\text { - } & \text { Transparency } \\
\text { - } & \text { Discourse } \\
\text { Preservation }\end{array}$ \\
\hline Warner (2005) & $\begin{array}{ll}\text { - } & \text { Certification } \\
\text { - } & \text { Registration } \\
\text { - } & \text { Awareness } \\
\text { - } & \text { Archiving }\end{array}$ \\
\hline Thompson (2005) & $\begin{array}{ll} & \text { Certification } \\
\text { - } & \text { Dissemination } \\
\end{array}$ \\
\hline Borgman (2007) & $\begin{array}{ll} & \text { Legitimisation } \\
\text { - } & \text { Dissemination } \\
\text { - } & \text { Access, preservation and curation. }\end{array}$ \\
\hline
\end{tabular}




\begin{tabular}{|l|l|}
\hline Source & Functions of research publishing system \\
\hline & \\
\hline
\end{tabular}

\subsection{Trends in Research Communication}

This section reviews literature on current trends in research communication. This will serve to build a case for this research investigation on factors affecting adoption of ICT for research communication by researchers in research institutions in Kenya, with a view to developing an ICT-enabled research communication model that can increase Kenya's participation in the global knowledge economy.

\subsubsection{Global trends in research communication}

There is growing dissatisfaction with the established research communication system, stemming from a variety of factors including rapidly rising subscription prices, concerns about copyright, latency between results and their actual publication, and restrictions on what can be published and how it can be disseminated. This has resulted into a global debate on how to remedy the system's deficiencies, and that debate has inspired concrete initiatives aimed at reforming the process to ensure access issues by seeking to alleviate two longstanding problems: the "serials crisis," which addresses the often prohibitive prices of journal publications that impede access to scholarly materials, and the "permissions crisis," which addresses the restrictions on use of publications once access has been obtained (Henry, 2003; Van de Sompel, et al. 2004).

At the beginning of the $21^{\text {st }}$ century, research communication is going through difficult times with most of the university presses and the commercial publishers who are still active in this field struggling to find ways to continue publishing scholarly research work (Darko-Ampem, 2003; Thompson, 2005; Borgman, 2007). The established scholarly communication system has not kept pace with revolutionary changes in research practice (Van de Sompel, et al. 2004). The question that arises is as Meadows (1998) poses, "How could communication of research be handled most efficiently as funding slowed?” (p. ix). He sees development of computers as a facilitator to rapid manipulation of large quantities of information and increasingly effective tools for the communication of research. Van de Sompel et al. (2004) see increased computer power, more pervasive computing, search technologies, collaboration, authentication, and security as the drivers of the likely technologies that may have an influence on the future of research communication. Increased computer power enables collaborative working, such as the Grid, virtual reality, data mining, and the fast exchange of more non-text 
materials. Pervasive computing includes mobile and wireless computing, wearable computers, speech recognition and personalisation (Van de Sompel, et al. 2004).

Roosendaal \& Geurts (1997) hold that scientific communication nowadays means electronic communication or electronic dissemination of "knowledge", which implies a virtual carrier for the virtual product, making use of a virtual memory. Atkins et al. (2003) envision "an environment in which raw data and results are easily shared, not just within a research group or institution but also between scientific disciplines and locations. There is an existing opportunity to share insights, software, and knowledge, to reduce wasteful recreation and repetition” (p. 12).

Rethinking and redesigning research organisations and processes to make best use of information communication technologies is requisite for success. Atkins et al. (2003) explain that this is not about "simply automating existing methodologies and practices" but advanced cyberinfrastrure which offers the potential to conduct new types of research in new ways (p.15).

Scholars/researchers can also use the new infrastructure to educate the next generations of scholars/researchers, using best techniques, spanning disciplinary boundaries, and democratising participation. It can enhance international collaboration and resource sharing. Cyberinfrastructure has great potential to empower people who, because of their physical capabilities, location, or history, have been excluded from the frontiers of scientific/scholarly research and education (Atkins, et al., 2003; Nentwich, 2005; Borgman, 2007).

The conference of the Association of Learned and Professional Society Publishers (ALPSP) held in London on 8 April 2005 addressed the topic of how scholarly publishing could operate and develop in an established web environment and the resolve was that the adoption of the Internet technology had the potential to bring new features and services to researchers (Ashling, 2005). The keynote speech outlined the development of a scholarly communication process that was no longer a simple linear chain from author (researcher) to publisher to reader, but one of constant refinement and improvement involving the author as a central player. There should be a move from "what researchers want" to "what researchers are doing” with existing electronic resources because understanding where usage had increased dramatically would provide invaluable information to publishers and to those assessing the quality of research at 
specific establishments. This is possible with the availability of automated tools coupled with better metadata (Ashling, 2005).

The practices of scholarship and research communication are changing rapidly as continuous improvements in computing and network technologies, digital data capture and storage facilities, and powerful data mining techniques enable researchers to engage in highly collaborative, network-based, and data-intensive research practices (Van de Sompel, et al., 2004; Nentwich, 2005; Warner, 2005; Borgman, 2007). Talking about the status quo of research in the era of ICT-based applications, Nentwich (2003) describes ICT-enabled scientific research (cyberscience) as a "moving target. There is no day without a new E-journal seeing the light of day, without new 'cyber-collaborations' or without a new piece of software which might change the way scholars work,” (p. 4). He describes cyberscience as "evolving and innovating along the path." He adds that researchers in all fields are trying to stay abreast of this moving target, but barely succeed because the empirical evidence contained in articles is outdated the minute they are published.

These dramatic changes in the nature of scientific research require correspondingly fundamental changes in the research communication system. Scholars and researchers alike deserve an innately digital, network-based scholarly communication system that is able to capture the digital scholarly record, make it accessible, and preserve it over time (Roosendaal \& Geurts, 1997; Kling \& McKim, 1999; Kling, 2004; Van de Sompel, et al., 2004; Nentwich, 2005; Warner, 2005). To record scholarship effectively, the notion of the unit of communication must be extended from the journal article to include rich media, datasets and software and also a system that provides for the early registration of communication units through the possibility of preservation separate from the conventional sequence of scholarly publication. This calls for a more flexible approach that would permit the composition of complex documents that aggregate and extend other complex documents (Warner, 2005). The result is that the traditional, linear, batch processing approach is now undergoing a process of continuous refinement as scholars/researchers write, review, annotate, and revise in near- real time using the Internet (Atkins, et. al., 2003; Warner, 2005; Borgman, 2007). In the near-term, the publication process will be characterised by semantically marked-up electronic complex documents as the masters, with display and print versions secondary (Warner, 2005). 
Thus, the linear information chain is being fundamentally transformed into an interactive communication network - exactly what is required to support the present societal demands for knowledge growth and management (Roosendaal \& Geurts, 1997).

There is, therefore, need to look at possible configurations of communication as communication can only be effective and efficient if its configuration appeals to the research community and the society at large (Roosendaal and Geurts, 1997; Kling, McKim et al., 2003; Kling, 2004; Borgman, 2007). Scholars need a communication system that helps them efficiently publish, access, reuse and assess the relevance and quality of information (Warner 2005). Ashling (2005) further stresses this when he urges publishers to listen to and observe how their customers use technology, since the most popular innovations of the digital era, for example, the Web, browsers, e-mail, instant messaging, texting, peer-to-peer file sharing, blogs, and wikis, have been userand communication-driven.

Thus, there is need to address the role of communication and its organisation in relation to the entire research process. Van de Sompel et. al. (2004) argue that the future research communication system should closely resemble and be intertwined with the research endeavour itself, rather than being its after-thought or annex. The same views are held by Borgman (2007), Kling, et al. (2003) and Casal (2007). It should comprise an interoperability substrate allowing flexible composition of value-adding services that, up to now, have been vertically locked in the journal publication milieu. In this loosely coupled system, the units of scholarly communication (i.e., data, simulations, informal results, preprints, etc.) could follow a variety of scholarly value chains in which each hub provides a service such as registering results, certifying their validity, alerting scholars to new claims and findings, preserving the scholarly record, and ultimately rewarding scholars for their work (Van de Sompel, et al. 2004). They see a unit of communication in a future scholarly communication system constituting the following:

- Datasets, simulations, software, and dynamic knowledge representations complex documents that flexibly aggregate the products of the scholarly endeavor, regardless of their format or location, which are recursively available for inclusion into other compound units. Such technology would provide for the reuse and derivation of existing results that is an integral part of the scholarly process 
- Early registration (and, ultimately, preservation) of all units in the system, regardless of their nature or stage of development. This would facilitate collaborative network-based endeavors and increase the speed of discovery. Preprints, raw datasets, prototype simulations, and the like should be afforded the ability to proceed through the scholarly value chain in the same manner that only journal publications are afforded in the current system (Van de Sompel, et al. 2004).

It is anticipated that such flexibility would empower individual research communities to decide which actions constitute registering a unit of communication, as well as what the community deems acceptable with respect to the timing of registration and how that relates to the quality of what is to be registered. In addition to facilitating an increased speed of discovery, a more flexible environment would allow scholars to officially incorporate into the system of communication materials that are currently largely living in a grey literature area (Van de Sompel, et al. 2004).

Table 2.2 below presents a summary of the trend in communication of research. 
Table 2.2: Changes in academia on the path to cyberscience (Nentwich, 2003, p. 25)

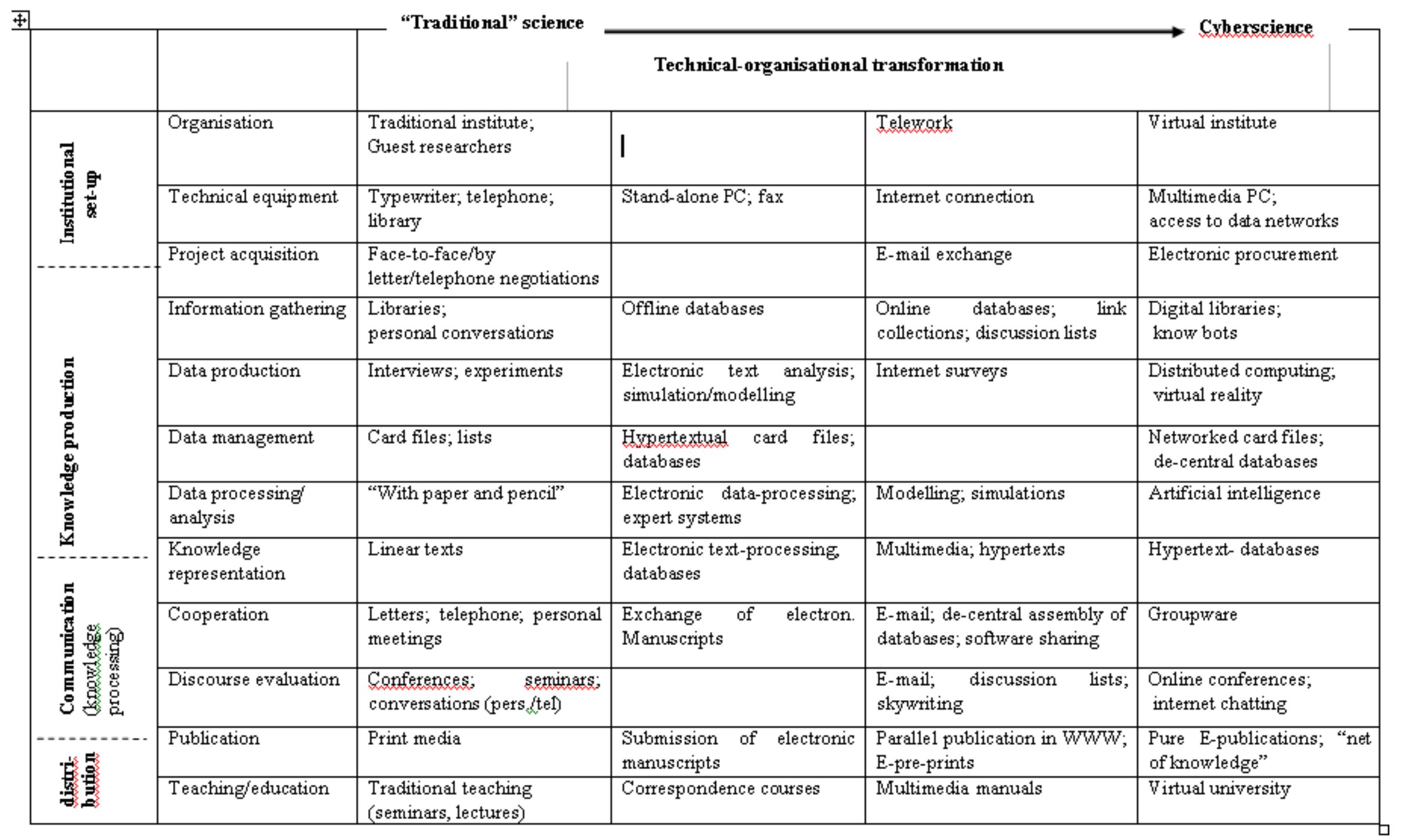




\subsubsection{Research communication in Africa}

The research community in the developing countries has had difficulty publishing in the mainstream established journals and the regional journals, which could have helped ease the problem of low and irregular distribution levels (Kirsop, 2002). In addition, journals from developing countries are poorly represented in international bibliographic databases. This, along with low print circulation, adds to the poor visibility of research published in journals from developing countries. This in turn leads to lower citations for the published articles, so that journals with a low impact factor continue to remain less subscribed and poorly visible; thus resulting to a circle of inaccessibility (Sahu, 2006; Sahu \& Chan, 2004), as illustrated in figure 2.2 below. They further argue that "The main problem in journal publishing in such countries is not poor circulation but poor submission," (p.60) which "fuels a vicious cycle" (Sahu \& Chan, 2004), as authors are reluctant to submit to journals with poor circulation because they want to be read. This lack of submission leads to irregular publication and subscription to journals, ultimately leading to invisibility.

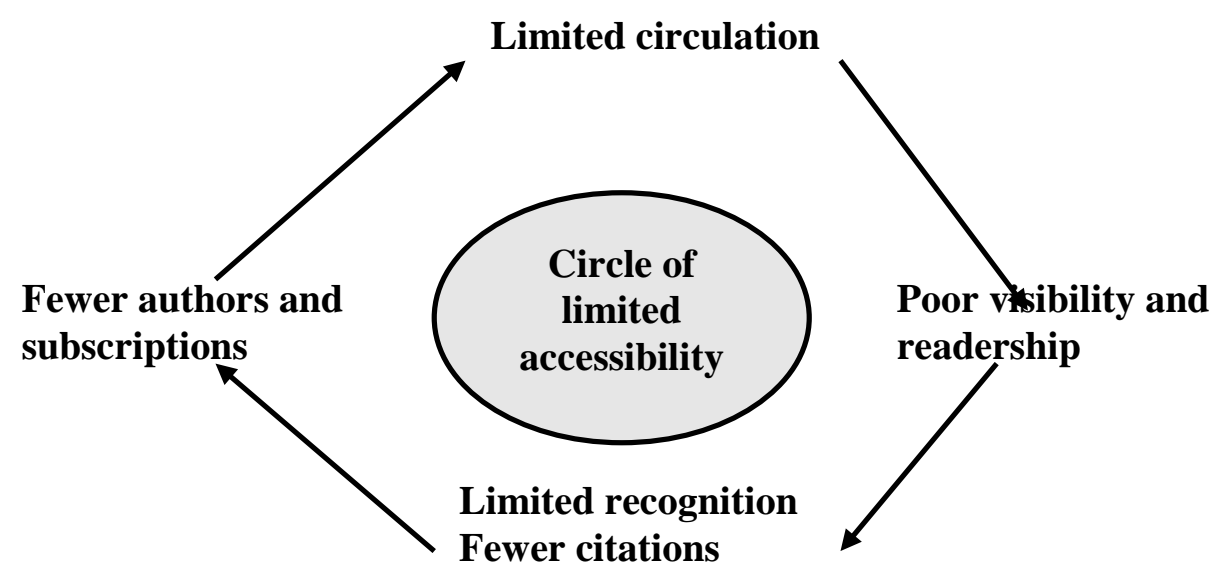

Figure 2.2: Limited visibility (Sahu, 2006)

This has resulted in a knowledge gap or digital divide that runs in three dimensions: South to North, North to South, and South to South. The South to North divide is caused by limited local resources in developing countries, limited ICT skills, a lack of access to technology and "in some quarters there is a perceived lack of credibility that we should overcome. Publications in the South are in some cases not valued as highly as those originating in the North” (Durrant, 2004, p. 122). 
The North - South gap has been a publishers' one arising from high printing and distribution costs that have made information from the North to the South too expensive a commodity for developing countries to afford (Durrant, 2004). "Reading material has become enormously expensive. There are very few universities in developing societies that can afford the scientific journals that are produced. These journals are enormously expensive, along with books" (Menon, 2004, p. 6). According to the ARL, costs of serials have risen by about $15 \%$ per year and are projected to rise by $1800 \%$ by 2020 (Kirsop, 2002).

Talking about ongoing scientific research publishing initiatives in developing countries, Sahu \& Chan (2004) say, "Many initiatives that focus on providing information, such as the Health InterNetwork Access to Research Initiative (HINARI), are concerned with information flows from the North to the South" and argue that "while that is very important, we must remember that much of the information created in the South is also important to the North as well as to the South, particularly in areas such as biodiversity and tropical and infectious diseases" (p.58). Consequently, research from the region has been largely unknown and unacknowledged, leading to a feeling of professional isolation (Kirsop, 2002; Sahu, 2006).

Richard Horton (1996) says, "The invisibility to which mainstream publishing condemns most third world research thwarts the efforts of poor countries to strengthen their indigenous science journals and with them the quality of research, in regions that most need them" (cited in (Kirsop, 2002). Durrant (2004) concurs with Horton: "the influx of information from the North to the South is in danger of damaging indigenous initiatives. Authors in the South are looking for recognition and reach just as authors in the North" (p.123). Munoz (2006), in an INASP discussion group in-debate entitled “Are local journals important?”, says: “In a world moving rapidly towards the knowledge-based economies, capacity building in science and technology is necessary and urgent for the developing countries. Local research publications are undoubtedly an efficient way to promote science and to support the dissemination of local research, where national bodies should become involved to strengthen these journals" (n.p).

Africa has not been spared, as Zell (1992) explains:

There has in the past been a tendency for academic institutions, booksellers, and individuals to view African books as part of a minority-taste ghettoized subculture, whereas of course, African publishing output needs to be known and disseminated as 
part of the mainstream. It should not be seen as peripheral - or of the "radical/alternative” or "exotic” variety (p.112).

Canagarajah (1996) talks of the “"nondiscursive' requirements” of academic publishing houses in the West, a "hidden publishing" agenda that makes it virtually impossible for researchers from the Third World to publish successfully in industrialised world, which leads to exclusion and marginalisation of peripheral (Third World) research. The same is echoed by Avgerou (2004) when she talks of the institutionalisation of the scientific processes by the developed world leading to "rituals of writing, referencing, and arguing” (p. 42), in which developing countries lack the capacity to take part and prove their point competently, as peer reviewers from the industrialised world would like to see. She talks of the existing publishing system as reifying information without addressing the context; yet scientific research is codified information whose legitimacy stems from different socio-economic-cultural contexts. She emphasises that it is "important to be able to unpack the black boxes of scientific data and be able to interpret them, understand their contextual nature and make choices that are contextually appropriate” (p. 42)(p. 42). Canagarajah (1996, p.21), like Avgerou (2004), calls for the need to situate knowledge and personalise it to accommodate views from the periphery, which, being critical of the centre (developed world), may expand and enrich narrow knowledge from the centre. Altbach (1996) picks up the same point and argues for a better balance of research agendas between researchers and users, strengthening of regional and international networks for sharing research, and inclusion of peripheral research communities in the international mainstream.

Kaniki (2004)(2004, p.83) highlights Internet connectivity constraints, commodification of knowledge, and problems of knowledge production and dissemination as issues that impact on the research community in Africa. He says institutions of higher learning are now being pushed to move away from basic research into the development of products that they can sell to maintain themselves. Because of the various pressures that these institutions face and the broad roles they are expected to play (teaching, research, and community development), little or no funds are allocated for research, knowledge production, or access to the latest information in various fields. For example, many African institutions of higher learning have not renewed their journal subscriptions for many years and have no research equipment or 
appropriate systems for managing research. This has resulted in research being pushed aside because of the difficulty in obtaining funds, creating a situation in which academics are simply teaching and, in many instances, indulging in consultancies rather than conducting research.

Another problem with research communication in Africa has been a lack of innovation policies, strategies and priorities to guide development and adoption of the same in the research publishing and communication process (Kaniki, 2004; Juma, 2008). Yet an effective research process requires an awareness of who is doing what, what kind of research is being conducted within a particular field, and what data and knowledge have been generated. These data and information form the basis for further research. Like their counterparts in the North, African researchers and research managers need to remain updated and networked with colleagues in the same field. However, African universities and research institutions, which Juma (2008b) says could be the continent's "gateways into the global knowledge economy for local diffusion of new technologies[,] ... remain digitally isolated from the rest of the world" (n.p). Lacking access to modern ICT, African universities were reported to "have the Internet capacity of a family home in Japan, which is equivalent of " 30,0000 people trying to use a single household connection” (Juma, 2008a).

The isolation of African scientists and lack of visibility of African science are recognised in the Association of African Universities (AAU) Strategic Plan 2003 - 2010 (Association of African Universities 2004). A conference report by AAU says Africa is suffering from an absence of data for critical analysis and proper planning, preventing and forecasting on issues such as rural development programmes and agriculture, malaria, floods, etc. It depicts African researchers as loners in a globalized world, “...sharing ideas among themselves is a missing link” (Association of African Universities, 2005, n.p).

In her presentation on "The Ptolemy Project: Delivering Electronic Health Information in East Africa," Beveridge (2004) stresses the need for local research in Africa. She says, "The health problems of Africa will be solved in Africa by Africans, for it is they who know the right questions to ask to get practical answers and find solutions that work. Yet how are they to do meaningful research if they cannot read what has previously been written on their 
topic?” (pp. 49-50). Juma (2008a, 2008b) holds that provision of low-cost, high speed Internet access to African universities could help Africa build the necessary capacity to address its own problems. He told the G8 summit that this would be "the most strategic investments that the G8 countries can make in Africa in the coming few years” (Juma, 2008a).

The availability of research data that can be appropriately contextualised is crucial for knowledge production. It is important to understand that factual data are relevant, and that African researchers and research managers should have access to them. The existing databases and new tools and techniques that allow data mining and pulling together the different sources of data must be interconnected and populated with relevant data from Africa itself, for it is critical that the African region contributes to the data generated around the world (Kaniki, 2004). That is why this study focused on investigating factors affecting ICTadoption as a first step towards coming up with a framework for ICT adoption, informed by the socio-cultural, institutional and technological contexts. It is hoped such a framework could serve to increase accessibility, visibility and Kenya's and Africa's participation in the global economy.

Developments in ICT and training, especially open access, could lead to improved networking and more rapid response to changing circumstances by African researchers and, hence, facilitate exploitation and utilisation of both international and local information resources: "These changes in ICT and the way information can be processed means that productivity in science should improve. We should be getting more results” (Cowan, 2004, p. 30). Munoz (2006) argues that the Internet is a good opportunity for local journals as it lowers the costs associated with paper publications and increases the effectiveness of scientific communication, giving visibility and accessibility to the articles. Thus, ICT-mediated research communication could help bridge existing disparities (Association of African Universities, 2004; Beveridge, 2004; Durrant, 2004; Juma, 2008a, 2008b; Kaduda, Olola, Oluoch \& Mwazi, 2005; Matsuura, 2004; Muinde, 2004; Musa et al., 2005; Sahu, 2006; Sahu \& Chan, 2004).

Open access has been said to confer visibility to research output by enabling researchers to communicate their results widely, quickly, and cheaply; assisting in connecting global 
research communities; and, providing the potential to foster partnerships and strengthen scientific cooperation South to South and South to North (Beveridge, 2004; Durrant, 2004; Sahu, 2006; Sahu \& Chan, 2004; Willinsky, 2006). Menon (2004) sees ICT-enabled research communication as having the potential to avail to African countries the existing and growing global reservoir of global knowledge while at the same time providing an opportunity for them to share valuable inheritance in content so far carried by oral tradition, community knowledge, and social interactions.

Nevertheless, Makhubela (2004) underscores the need for an analysis and understanding of the social setting of research institutions for these exist and operate within a context with set values and norms specific to that social setting and which are also intrinsically linked to the political economy and power relations in the South-North and South-South divide. She further argues that the real challenge in terms of free ICT-mediated research communication is not "physical access and high-level connectivity to the Internet... The broader question of how much is technology a tool for development still remains” (p.79). Her views align with Kaniki (2004, p. 83) who argues, “Information needs, even among people, organizations, and countries of the same group or class, are context-dependent.” These views indicate a gap in knowledge in ICT-enabled research communication and provide a justification for this study which sought to investigate the factors that affect ICT adoption and use in scientific research communication by researchers in Kenya, with a view to coming up with a contextual framework for ICT-enabled scientific research communication by researchers in Kenya. This may lessen resistance and align ICT adoption with needs and priorities of researchers and, therefore, facilitate greater participation by researchers in Kenya in the local and global knowledge network and ultimately increase visibility of Kenyan scientific research outputs...

Thus, the issue of access to research outputs in Africa, Kenya in particular, has multiple aspects. Consequently, workable solutions will necessarily have to include all the relevant dimensions. It is only then that ICT as a tool may help rid off "knowledge imperialism" or domination by the centres in the developed world and give visibility to the African research; thus, bridging the gap between the periphery and the centre (Altbach, 1996; Avgerou, 2004; Canarajah, 1996; Mazrui, 1978; Muthayan, 2004; Teferra, 2003).2.3.3 Research Communication in Kenya 
Kenya’s 1988 'Presidential Working Party', set up to review the quality and relevance of education for the next decade and beyond, recommended there was need for university and higher education in general to cultivate the development of science and technology and research in all disciplines. The committee gave two important objectives of the public universities as:

- To develop, advance, preserve and disseminate knowledge and to stimulate intellectual life;

- To provide through research and consultancy, knowledge, skills and services to the community by helping solve problems facing the community (Republic of Kenya, 1988, p. 69).

Then in 2003, the Technical Working Group on the Education Sector Review and Development gave the objectives of University Education as, among others, to:

- develop in students and scholars the ability to think independently, critically and creatively;

- adapt, develop, advance, preserve and disseminate desirable values and to stimulate intellectual life (including the spirit of services to others);

- educate and train the high level human capital needed for accelerating development through industrialisation of the economy;

- nurture the internalisation of universal knowledge, including key technological advances, with a view to harnessing it for national development; and

- provide through basic and applied research, knowledge, skills and services that help solve the problems facing society (Government of Kenya, 2003b, p. 75).

To date, these objectives are yet to be achieved as witnessed in recent policy documents (Government of Kenya, 2003a, 2003b, 2005a, 2005b). The realisation of these objectives respectively spells the need for good exchange of research information and knowledge (Adeya \& Oyelaran-Oyeyinka 2002; Duque, et al. 2005). This in turn requires a wellestablished communication system and collaboration networks, which are currently nonexistent. 
Chakava (1992) says, "Very little has been written on the Kenyan publishing industry even though it is one of the most important in Africa” (p. 119). The Kenyan publishing industry flourished in the period 1970 - 1977 which saw the formation of two professional associations in 1971 - the Kenya Publishers' Association and the Kenya Booksellers and Stationers Association - to accommodate all the nationalities involved in the book trade. This was a period of great economic growth with the Kenya shilling very strong. However, from 1977 to date, the Kenya publishing industry has experienced lean years. This was partly caused by the closure of the Kenya - Tanzania border which cut off access to south markets in Zambia and Malawi. There was little business with neighbouring Uganda following Idi Amin's coup d'etat of 1971. In addition, the uncertainty surrounding president Moi's succession in 1978 and a coup attempt in 1982 have made it worse (Chakava, 1992).

Scholarly publishing was the hardest hit. Chakava (1996) describes the university in Kenya as an institution that has been "largely politicized, and a majority of university professors are absorbed into the state system. Creativity is stifled through the curtailment of literary seminars, journals, and writers' workshops, and a general lack of facilities or incentives to promote and reward academic excellence. There is lack of an intellectual culture and debate on important issues of the day,” (p.14). The same is echoed by Ondari-Okemwa ( (2007); Omwoha and Gakahu (2009) and Nyariki (2008).

Journals, though an important as a forum for exchanging peer views on the latest developments in the world of knowledge, have been almost non-existent, as Chakava (1992) points out in his article, “Kenyan Publishing: Independence and Dependence,” which concludes "by having a brief look at the current journal-publishing situation in Kenya, even if it is to draw attention to the non-existence of journals” (p.136). Most of the country’s journals were launched in the period between 1965 and 1975, mostly published by the East African Literature Bureau (EALB) and EAPH (East African Publishing House). However, the folding up of the bureau in 1977 and the death of EAPH itself in 1987 "put the final nail in the coffin of journal publishing in Kenya” (Chakava, 1992, p. 137).

The lean years of 1980s also affected Kenyan universities and research institutions. The devaluation of the Kenyan currency cost the university libraries about $30 \%$ of their 
purchasing power of foreign journals (Mutula, 2001b, p.156). Libraries lacked reference materials which are key in research communication (Waast, 2005). At Kenyatta University, for example, by 2003 the library's serials collection was reduced to one "core" print journal per department (Muinde, 2004). Willinsky (2006) recounts his observation during a visit of University of Nairobi library in 2003 of "seven well-crafted wooden racks for displaying current periodicals, all of which stood empty, with not a journal on display or a back issue stored beneath the hinged racks” (p.100). This reduced institutions' capacities to support both research and its dissemination through publications, resulting in the inaccessibility and invisibility of the Kenyan scholarly research output.

Of the six public universities in Kenya, only University of Nairobi has a university press - set up in 1983 with a grant from BAT (British American Tobacco) (Chakava, 1992; DarkoAmpem, 2003). However, up until 1990, the press had published only one title, owing to management problems (Chakava, 1992). Although there has been some improvement in scholarly publishing in Kenya since then, this is still insignificant compared to countries in North America, Europe and Asia (Nyariki, 2008; Omwoha \& Gakahu, 2009; OndariOkemwa, 2007; Tijssen, 2007; UIS, 2005). For example, from 1997-2007, Kenya produced only 6661 publications. This compared insignificantly with what was produced in 2006 in USA - over 100,000, England - 97,904, Germany - 94,899, Japan -89,507, Canada - 59,271, South Korea - 31,268, India - 30,744, Russia - 23,558, Brazil -21,450, Israel - 15,564 and Mexico - 10,948 (Ondari-Okemwa, 2007; UIS, 2005).

Declining incomes and morale in the universities led to diminished interest in, and ability to support, the professional associations among academics; yet, it was these associations that were responsible for publishing many national and sometimes regional and continental disciplinary journals. Researchers and scholars lacked incentives (Ondari-Okemwa, 2007; Tijssen, 2007). Moreover, universities and research institutions lacked equipped laboratories and internet connectivity (Ondari-Okemwa 2007; Waast, 2002). As a result, scholarship production and productivity for some disciplines went down (Zeleza, 1998). Whole areas of expertise like agricultural sciences virtually disappeared (Ondari-Okemwa, 2007; Waast, 2005). 
A study of Kenyan and Nigerian universities found that research activities in Kenya took only $23 \%$ of lecturers' time, even though $73 \%$ of the respondents had some current research interest (Adeya \& Oyelaran-Oyeyinka, 2002). It also established most of the researchers still relied on traditional sources of information such as technical and research journals and seminar proceedings in hardcopy, while publishing research work was still through snail-mail publishing process. In addition, local networking, even with peers in Africa, was poor and researchers knew little about the current research interests within the continent.

Ochuodho and Matunga (2005) also highlight the problem of inaccessibility of Kenyan research output. They recount their experience when writing their article on Civil Society Involvement in ICT Policy Making: "During the literature review, it emerged that there was very little by way of published materials on the Kenyan ICTs scene generally, and particularly on policy formulation," (p.76). They also report that "statistics and models on ICT in Kenya are virtually non-existent” (p.80) and recommend for strengthening of research institutions to make it possible for them to collect and analyse data in this area to inform planning and public policy.

During the first National ICT Convention held in Safari Park Nairobi between 23-25 March 2004, the participants collectively observed that research was required to improve the information and data gaps (Nduati \& Bowman 2005). Among other recommendations, the participants recommended the Government implement an ICT for development and growth curriculum at all levels (primary, secondary and tertiary). Nduati \& Bowman (2005) conclude by recommending, "Kenya must invest in more education for computer scientists and to ensure that Kenyan higher education is linked to the needs of the indigenous private sector" (p.67). This requires that academia and researchers be exposed to ICT-enabled communication networks for efficient and effective collaboration.

The studies cited above pointed to a gap in knowledge concerning ICT research and also communication of research outputs in Kenya. This justified the necessity for this study that sought to establish factors that affected the adoption of ICT for research communication with a view to develop a contextual framework for ICT-enabled research communication. 
Despite the difficult times facing research communication in Kenya, there were bright prospects for future publishing in this area. Gideon S. Were Press initiated and continues to publish two journals, the Journal of East African Research and Development and the TransAfrican Journal of History. There is also Academy Science Publishers, which has published Innovation and Discovery and the African Centre for Technology Studies (ACTS) Press. These would fill a gap long felt by all scientists in Africa, who hitherto had no forum for exchanging new ideas and discoveries. There were also other initiatives using the open access to enhance the accessibility and visibility of African scholarly research. These included:

- the International Network for the Availability of Scientific Publications Programme for Enhancement of Research Initiatives (INASP/PERI), which has negotiated agreements with EBSCO (a major journal subscription service), Springer, OUP, Blackwell, and others, to enable electronic access to journals and journal abstracts from Africa through the African Journals Online (AJOL) project (Durrant, 2004; Muinde, 2004; Rosenberg, 2005; P Smart, 2003, 2005; Willinsky, 2006).

- The Bioline International hosted at the University of Toronto, whose goal is "to improve the visibility, accessibility and subsequent impact of research that would otherwise be 'lost' because few research libraries subscribe to developing counties' journals despite their importance. ... Our experience suggests that open access not only enables free flow of ideas, it ensures more equitable scientific developments and their applications to social needs, including those of the developing countries” (Willinsky, 2006, p. 104).

- The African Virtual University (AVU) launched in 1991 in Nairobi. Utilising satellite technology, AVU was able to serve students through 34 sites (with over 1000 computers) in 19 African countries during its "proof of concept" stage, with courses in technology, engineering, business, and the sciences sourced from leading universities in North America and Europe. Having moved out of its pilot stage as a World Bank project, this independent intergovernmental organisation now offers access to 1000 online journals through its digital library and has helped institutions across Africa to set up AVU learning centres with high-speed connectivity to the Internet (AVU website < $\underline{w w w}$.avu.org>; Willinsky, (2006)). The AVU library is devoted to "breaking through the information access barriers" as this "global platform” also seeks to make African content available to the world, while improving African access to resources (Kamau, 2001). She argues, 
"market information products from the developed world... fail to recognize the potential that local content has as a part of a global knowledge.”

The background given above necessitates the need for local research and, consequently, further investigation to determine the organisational, social, cultural and technological factors that affect the adoption of ICT to the research communication process by researchers. This would inform the development of a contextual framework for ICT-enabled research communication that can facilitate access and visibility of the Kenyan research output.

\subsection{ICT and scientific research communication}

“As information and communication technologies have become essential tools for science, governments need to understand their role in the science system in order to develop appropriate science policies” (OECD 1998, p.189)

(OECD, 1998, p.189)

Atkins, et al. ( 2003) hold that the Internet and the web were invented to support the work of researchers by enabling the rapid communication of ideas, the sharing of resources, and remote access to data. Increasing networking and interoperability of information formats and access make high-quality remote collaboration feasible and facilitate inexpensive storing and archiving of hundreds of thousands of terabytes of scientific information and data (Nentwich, 2005; Van de Sompel, et al., 2004; Warner, 2005). Most researchers would not be able to function without email or access to the web, for this would mean fewer contacts with distant, especially international, scientists and inability to stay on the cutting edge (Atkins, et al., 2003).

The advent of ICT has the potential to affect almost every aspect of research activity (Nentwich, 2003). The Internet enables new methods of scientific communication that greatly speed up the advancement of science and scholarship. It saves on research time that would otherwise be spent exploring the abundance of information and data; thus, improving the effectiveness and efficiency of the scientific communication system. This calls for the envisioning of a strategic — or structural — transformation of the scientific communication network, as opposed to a more tactical —or mechanical — transformation of the information chain that can only result in improvement to the existing system (Borgman, 2007; Kling, et al., 2003; Nentwich, 2005; Roosendaal \& Geurts, 1997; Van de Sompel, et al., 2004; Warner, 2005). 
Technology serves as an important driving force as it enables further developments in the research process and empowers researchers to induce a structural change in their communication configuration, which can lead to advancement of knowledge. It has also been seen to lead to further empowerment of the key stakeholders - the authors and the readers in scientific communication (Borgman, 2007; Roosendaal \& Geurts, 1997). There is, therefore, need to identify the enablers and barriers of adoption of ICT to research communication as a first step towards developing a model to maximise exploitation of the same to give accessibility and visibility to scientific research.

The emerging technologies like the Internet have facilitated the information networks that are encouraging changes in the structure of information flow in Africa. Access facilitates a diffusion of quality education (UNECA, 1996)(UNECA 1996). The explosion of information and scientific knowledge has made it practically impossible to stock all published materials in hard copy even by the most committed and affluent Western repositories, and the funds to secure the publications and even the space to display and store them have become limited (Teferra, 1998, 2003). Access to the Internet for African scholars and editors will not only alleviate their isolation from the heartland of the knowledge base in the West, but also bring them together to address common related regional issues (Adewuyi, 2008; Casal, 2007; Juma, 2008a, 2008b, 2002). In addition, African scholars will be able to surf the information superhighway for current information, thereby promoting their scholarly work, their intellectual vitality, knowledge production, and dissemination (Teferra, 1998, 2003). Nevertheless, there are issues associated with scientists' communicative ability that need addressing in order to reap the full benefits that come with ICT (Adewuyi, 2008; Alo, 2003; Gewin, 2009).

Africa is recognising that scientific research and technology innovations are its prime productive force in adding value to its raw material and immensity of natural resources. Consequently, during the AAU November 2005 conference in Tunisia on African Research and Education Networking Infrastructure, participants pressed for the necessary level of bandwidth to support education, research and outreach locally, regionally and internationally. Referring to Africa as an isolated continent, whose potential remains unrealised owing to its universities' and research institutes' digital isolation from the rest of the world, Juma (2008a, 2008b) appeals to leadership in Africa and elsewhere in the world to help in increasing bandwidth to enhance net access to research information. 
The Government of Kenya views information communication technology as a critical form of wealth and an ICT literate workforce as the foundation on which Kenya can acquire the status of a knowledge economy. It has declared that innovation, research, development, ICT, and science and technology will form one of the key pillars of education and training. It is committed to exploiting the benefits of ICT to improve the research process and the dissemination of the research output to the ultimate beneficiaries (Government of Kenya, 2003a, 2003b, 2005a, and 2005b). The Sessional Paper Number 1 of 2005 and the KESSP policy framework (Government of Kenya, 2005b) have outlined the government's commitment to promoting the expanded use of ICT as a tool for effective management, research and development at all educational levels, and use of Internet for education, training and research and promotion of Open Distance Education (ODE) and virtual institutions, particularly in higher education and training.

The Report of the National Conference on Education and Training (Nov 27- 29 2003) made the following recommendations pertaining to research communication and ICT adoption, which make this study relevant:

- Universities should institutionalise life-long learning; allow specialisation and exchange of staff and sharing of between universities and industry to enhance relevance of and quality of programmes.

- The government should set aside a percentage of Gross Domestic Product (GDP) for research in universities and universities to ensure that research results are utilised for national development.

- University Councils should ensure that access to the Internet by universities is free and that all staff have access to ICT facilities. In addition, the Commission for Higher Education (CHE) should ensure that all universities have and maintain databases, which can be used by other institutions to enhance collaboration and mutual support.

- The Ministry of Education, Science \& Technology's (MOEST) mission statement should be reviewed to facilitate mainstreaming of ICT in the education system and thus enhance national development. It should read, "To provide, promote and coordinate life-long education, training and research for sustainable development (in the emerging Global 
Information Society)”. The mission statement echoes the key role of research in development and recognises the facilitating role of ICT in research and education.

- The MOEST should provide policy guidelines that ensure that ICT is mainstreamed into the curriculum at all levels and that the necessary human resource is developed.

- The MOEST should establish a publication for research findings in ICT in education and the related sectors.

- The policy on ICT development must acknowledge the importance of local content and local languages, and therefore support the New Partnership for Development (NEPAD) recommendations for developing ICT platforms and content in local languages.

- It is important to build upon what has worked in Kenya in order to strengthen existing institutions and share experiences and resources through the "centres of convergence" concept. This concept entails a number of universities pooling together to offer open and distant learning. Further, it is proposed that the Kenya Education Network (KENET) become a National Education Exchange Node for use by public and private educational institutions at all levels.

- Collaboration by educational institutions in Kenya with the African Virtual University (AVU) should be acknowledged as a success story for centralising the development of instruction modules and courses, with a view to distributing them across a large number of campuses and learners.

- The Government through the establishment and enforcement of intellectual property rights should provide the recognition and protection of local innovations in ICT.

- The Kenya Publishers Association, with the support of MOEST and Kenya Institute of Education (KIE), should convene a high level meeting to address packaging of content in electronic format for the use in the educational sector. Intellectual Property Rights will also need to be addressed in this context.

- The MOEST should strenuously promote the expanded use of ICT as a tool for effective management, research \& development at all educational levels, the utilisation of digitised local content and other quality and relevant multimedia content, and the maximisation of the Internet for research and education. 
- Promotion of local content using the full spectrum of ICT, from print media to the Internet, adoption and adaptation and delivery of curricula through the use of ICT as part of curriculum integration projects.

- The MOEST should support the implementation, continuous development, application research and monitoring of open source technologies for use in education (Government of Kenya, 2003b, pp.30, 39-44) (Government of Kenya 2003, pp.30,39-44).

However, despite claims of the benefits associated with access to ICT, especially the Internet and WWW, "there is little research available to provide data in support of claims that progress is being made in the efforts combating information poverty in SSA [Sub-Saharan Africa] ... While quantitative data are available to show increases in ICT usage, teledensity, and mobile access, much work still remains in understanding progress toward bridging the digital divide, particularly from user’s perspective” (Gebremichael \& Jackson, 2006, p,272). Gebremichael and Jackson (2006) recommend further research to determine how SubSaharan Africa user groups interact within this ever-changing environment in order to provide a user-centred framework for developing and building ICT in the region( p.277). This justified this qualitative study on factors affecting adoption, diffusion and use of ICT by researchers in communication of research output in Kenya, a Sub-Saharan Africa country, with a view to develop a contextual framework for ICT-enabled research communication to facilitate availability of research findings to inform policy deliberations and help shed light on other developmental concerns.

\subsection{Chapter Conclusion}

Having looked at the literature on trends in research communication, ICT and development, the next chapter looks at possible theoretical frameworks that could guide the study in responding to the research questions in order to meet the objective of the research. This chapter has already revealed there is need for an understanding of the socio-cultural, institutional and the technological environment to inform the development of a contextual ICT adoption framework that reflects the needs of scientific researchers in Kenya. Theory is important, for it adds to the breadth of the research (Silverman, 2001) by broadening the world view. It guides examination of practice, thus, assisting in understanding the gaps in the phenomena being investigated. 


\title{
CHAPTER 3
}

\section{THEORETICAL FRAMEWORK}

\begin{abstract}
"When basic technologies are systematically introduced to solve local problems in the right cultural contexts, meaningful exposure to technology would come about, and more advanced technologies would be embraced and applied" (Musa, et al., 2005)
\end{abstract}

\subsection{Chapter Overview}

This study sought to investigate the barriers and enablers to ICT-enabled scientific research communication in Kenya with a view to develop a contextual framework to guide the adoption of ICT for research communication. The previous chapter has reviewed literature to establish gaps in ICT-enabled research communication and pointed to the need for a contextual framework to guide the adoption of ICT for research communication. This chapter further reviews literature on available technology diffusion and acceptance models with a view to coming up with a preliminary model that served as lenses through which the researcher probed for contextual information on factors affecting ICT-mediated research communication in Kenya. The chapter briefly highlights the issues addressed by various Diffusion of Innovation theories and moves on to give a justification for the theories chosen to guide the data collection to answer the research questions. This is followed by a review of how the selected theories are applied in IS studies, and presentation of the preliminary framework.

\subsection{Relevant Technology Diffusion and Acceptance Models}

Research in information technology (IT) acceptance has yielded many models, each with different sets of acceptance determinants, as illustrated in table 3.1 below. The table highlights the core constructs addressed by the various models that helped in determining the models adopted in this study. 


\begin{tabular}{|c|c|c|}
\hline \multicolumn{3}{|l|}{ Models and Theories of Individual Acceptance } \\
\hline Theory of Reasoned Action (TA) & Core Constructs & Definitions \\
\hline \multirow{2}{*}{$\begin{array}{l}\text { Drawn from social psychology, TRA is one of the most } \\
\text { fundamental and influential theories of human behaviour. It has } \\
\text { been used to predict a wide range of behaviours. Davies et al. } \\
\text { (1989) applied TRA to individual acceptance of technology and } \\
\text { found that the variance explained was largely consistent with } \\
\text { studies that had employed TRA in the context of other } \\
\text { behaviours. }\end{array}$} & Attitude toward behaviour & $\begin{array}{l}\text { "an individual's positive or negative feelings (evaluative effect) about } \\
\text { performing the target behaviour" (Fishbein and Ajzen 1975, p. 216). }\end{array}$ \\
\hline & Subjective norm & $\begin{array}{l}\text { "the person's perception that most people who are important to him think } \\
\text { he should or should not perform the behaviour in question (Fishbein and } \\
\text { Ajzen 1975, p. 302). }\end{array}$ \\
\hline \multicolumn{3}{|l|}{ Technology Acceptance Model (TAM) } \\
\hline \multirow{2}{*}{$\begin{array}{l}\text { TAM is tailored to IS contexts, and was designed to predict } \\
\text { information technology acceptance and usage on the job. } \\
\text { Unlike TRA, the final conceptualization of TAM excludes the } \\
\text { attitude construct in order to better explain intention } \\
\text { parsimoniously. TAM2 extended TAM by including subjective } \\
\text { norm as an additional predictor of intention in the case of } \\
\text { mandatory settings (Venkatesh and Davis 2000). TAM has } \\
\text { been widely applied to a diverse set of technologies and users. }\end{array}$} & Perceived Usefulness & $\begin{array}{l}\text { "the degree to which a person believes that using a particular system would } \\
\text { enhance his or her job performance" (Davis 1989, p.320). }\end{array}$ \\
\hline & Perceived Ease of Use & $\begin{array}{l}\text { "the degree to which a person believes that using a particular system would } \\
\text { be free of effort" (Davis 1989, p.320). }\end{array}$ \\
\hline \multicolumn{3}{|l|}{ Motivational Model (MM) } \\
\hline \multirow{2}{*}{$\begin{array}{l}\text { A significant body of research in psychology has supported } \\
\text { general motivation theory as an explanation for behaviour. } \\
\text { Several studies have examined motivational theory and adapted } \\
\text { it to specific contexts. Vallerand (1997) presents an excellent } \\
\text { review of the fundamental tenets of this theoretical base. } \\
\text { Within the information systems domain, Davis et al. (1992) } \\
\text { applied motivational theory to understand new technology } \\
\text { adoption and use (see also (Venkatesh and Speier 1999) ). }\end{array}$} & Extrinsic Motivation & $\begin{array}{l}\text { The perception that the users will want to perform an activity "because it is } \\
\text { perceived to be instrumental in achieving valued outcomes that are distinct } \\
\text { from the activity itself, such as improved job performance, pay, or } \\
\text { promotions" (Davis, Bagozzi et al. 1992, p.1112). }\end{array}$ \\
\hline & Intrinsic Motivation & $\begin{array}{l}\text { The perception that users will want to perform an activity "for no apparent } \\
\text { reinforcement other than the process of performing the activity per se" } \\
\text { (Davis, Bagozzi et al. 1992, p.1112). }\end{array}$ \\
\hline \multicolumn{3}{|l|}{ Theory of Planned Behaviour (TPB) } \\
\hline $\begin{array}{l}\text { TPB extended TRA by adding the construct of perceived } \\
\text { behavioural control. In TPB, perceived behavioural control is }\end{array}$ & Attitude toward behaviour & Adapted from TRA \\
\hline
\end{tabular}




\begin{tabular}{|c|c|c|}
\hline Theory of Reasoned Action (TA) & Core Constructs & Definitions \\
\hline & Subjective norm & Adapted from TRA \\
\hline & $\begin{array}{l}\text { Perceived } \\
\text { control }\end{array}$ & $\begin{array}{l}\text { "the perceived ease or difficulty of performing the behaviour" (Ajzen 1991, } \\
\text { p. 188). In the context of IS research, "perceptions of internal and external } \\
\text { constraints on behaviour" (Taylor and Todd 1995, p. 149). }\end{array}$ \\
\hline \multicolumn{3}{|l|}{ Combined TAM and TPB (C-TAM-TPB) } \\
\hline \multirow{4}{*}{$\begin{array}{l}\text { This model combines the predictors of TPB with perceived } \\
\text { usefulness from TAM to provide a hybrid model (Taylor and } \\
\text { Todd 1995) }\end{array}$} & Attitude toward behaviour & Adapted from TRA/TPB. \\
\hline & Subjective norm & Adapted from TRA/TPB. \\
\hline & $\begin{array}{l}\text { Perceived behavioural } \\
\text { control }\end{array}$ & Adapted from TRA/TPB. \\
\hline & Perceived usefulness & Adapted from TAM. \\
\hline \multicolumn{3}{|c|}{ гететveu userumess } \\
\hline \multirow{5}{*}{$\begin{array}{l}\text { Derived largely from Triandis' (1977) theory of human } \\
\text { behaviour, this model presents a competing perspective to that } \\
\text { proposed by TRA and TPB. Thompson, Higgins, \& Howell } \\
\text { (1991) adapted and refined Triandis' model for IS contexts and } \\
\text { used the model to predict PC utilization. However, the nature } \\
\text { of model makes it particularly suited to predict individual } \\
\text { acceptance and use of a range of information technologies. } \\
\text { Thompson, et al. (1991) sought to predict behaviour rather than } \\
\text { intention; however, in keeping with the theory's roots, the } \\
\text { Unified Theory of Acceptance and Use of Technology } \\
\text { (UTAUT) by V Venkatesh, et al. (2003) has examined the } \\
\text { effect of these determinants on intention to facilitate fair } \\
\text { comparison of the different models. }\end{array}$} & Job-fit & $\begin{array}{l}\text { "the extent to which an individual believes that using [a technology] can } \\
\text { enhance the performance of his or her job" (Thompson, Higgins et al. } \\
\text { 1991). }\end{array}$ \\
\hline & Complexity & $\begin{array}{l}\text { Based on Rogers and Shoemaker (1971), "the degree to which an } \\
\text { innovation is perceived as relatively difficult to understand and use" } \\
\text { (Thompson, Higgins et al. 1991, p. 128) }\end{array}$ \\
\hline & Long-term consequences & $\begin{array}{l}\text { "Outcomes that have a pay-off in the future" (Thompson, Higgins et al. } \\
\text { 1991, p. 129) }\end{array}$ \\
\hline & Affect towards use & $\begin{array}{l}\text { Based on Triandis, affect toward use is "feelings of joy, elation, or } \\
\text { pleasure, or depression, disgust, displeasure, or hate associated by an } \\
\text { individual with a particular act" (Thompson, Higgins et al. 1991, p. 127) }\end{array}$ \\
\hline & Social factors & $\begin{array}{l}\text { Derived from Triandis, social factors are 'the individual's internalization of } \\
\text { the reference group's subjective culture, and specific interpersonal } \\
\text { agreements that the individual has made with others, in specific social } \\
\text { situations" (Thompson, Higgins et al. 1991, p. 126) }\end{array}$ \\
\hline
\end{tabular}




\begin{tabular}{|c|c|c|}
\hline \multicolumn{3}{|l|}{ Models and Theories of Individual Acceptance } \\
\hline \multirow[t]{2}{*}{ Theory of Reasoned Action (TA) } & Core Constructs & Definitions \\
\hline & Facilitating conditions & $\begin{array}{l}\text { Objective factors in the environment that observers agree make an act easy } \\
\text { to accomplish. For example, returning items purchased online is facilitated } \\
\text { when no fee is charged to return the item. In an IS context, "provision of } \\
\text { support for users of PCs may be one type of facilitating condition that can } \\
\text { influence system utilization" (Thompson, Higgins et al. 1991, p. 129) }\end{array}$ \\
\hline \multicolumn{3}{|l|}{ Diffusion of Innovation Theory (DOI) } \\
\hline \multirow{7}{*}{$\begin{array}{l}\text { Grounded in sociology, DOI (Rogers 2003) has been used since } \\
\text { the 1960s to study a variety of innovations, ranging from } \\
\text { agricultural tools to organisational innovation (Tornatzky and } \\
\text { Klein 1982). Within information systems, Moore and Benbasat } \\
\text { (1991) adapted the characteristics of innovations presented in } \\
\text { Rogers and refined a set of constructs that could be used to } \\
\text { study individual technology acceptance. Moore and Benbasat } \\
\text { (1996) found support for the predictive validity of these } \\
\text { characteristics (see also (Agarwal and Prasad 1997; Agarwal } \\
\text { and Prasad 1998; Karahanna, Straub et al. 1999; Plouffe, } \\
\text { Hulland et al. 2001). }\end{array}$} & Relative advantage & $\begin{array}{l}\text { "the degree to which an innovation is perceived as being better than its } \\
\text { precursor" (Moore and Benbasat 1991, p. 195). }\end{array}$ \\
\hline & Ease of use & $\begin{array}{l}\text { "the degree to which an innovation is perceived as being difficult to use" } \\
\text { (Moore and Benbasat 1991, p. 195). }\end{array}$ \\
\hline & Image & $\begin{array}{l}\text { "The degree to which use of an innovation is perceived to enhance one’s } \\
\text { image or status in one’s social system” (Moore and Benbasat 1991, p. 195) }\end{array}$ \\
\hline & Visibility & $\begin{array}{l}\text { The degree to which one can see others using the system in the organization } \\
\text { (adapted from Moore and Benbasat (1991). }\end{array}$ \\
\hline & Compatibility & $\begin{array}{l}\text { "the degree to which an innovation is perceived as being consistent with the } \\
\text { existing values, needs, and past experiences of potential adopters" (Moore } \\
\text { and Benbasat 1991, p.195). }\end{array}$ \\
\hline & Results demonstrability & $\begin{array}{l}\text { "the tangibility of the results of using the innovation, including their } \\
\text { observability and communicability" (Moore and Benbasat 1991, p. 203). }\end{array}$ \\
\hline & Voluntariness of use & $\begin{array}{l}\text { "the degree to which use of the innovation is perceived as being voluntary, } \\
\text { or free will" (Moore and Benbasat 1991, p. 195). }\end{array}$ \\
\hline \multicolumn{3}{|l|}{ Social Cognitive Theory (SCT) } \\
\hline \multirow{5}{*}{$\begin{array}{l}\text { One of the most powerful theories of human behaviour is social } \\
\text { cognitive theory (see (Bandura 1986)). Compeau and Higgins } \\
\text { (1995) applied and extended SCT to the context of computer } \\
\text { utilization (see also Compeau, Higgins, \& Huff (1999)). } \\
\text { Compeau and Higgins (1995) model studied computer use but } \\
\text { the nature of the model and the underlying theory allow it to be } \\
\text { extended to acceptance and use of information technology in } \\
\text { general. The original model of Compeau and Higgins (1995) } \\
\text { used usage as a dependent variable but in keeping with the } \\
\text { spirit of predicting individual acceptance, Venkatesh et al. } \\
\text { (2003) in UTUAT model examine the predictive validity of the } \\
\text { model in the context of intention and usage to allow a fair } \\
\text { comparison of the models. }\end{array}$} & $\begin{array}{ll}\text { Outcome } & \text { expectations- } \\
\text { Performance } & \end{array}$ & $\begin{array}{l}\text { The performance - related consequences of the behaviour. Specifically, } \\
\text { performance expectations deal with job-related outcomes (Compeau and } \\
\text { Higgins 1995). }\end{array}$ \\
\hline & $\begin{array}{l}\text { Outcome expectations - } \\
\text { Personal }\end{array}$ & $\begin{array}{l}\text { The personal consequences of the behaviour. Specifically, personal } \\
\text { expectations deal with the individual esteem and sense of accomplishment } \\
\text { (Compeau and Higgins 1995). }\end{array}$ \\
\hline & Self-efficacy & $\begin{array}{l}\text { Judgement of one’s ability to use a technology (e.g., computer) to } \\
\text { accomplish a particular job or task. }\end{array}$ \\
\hline & Affect & An individual's liking for a particular behaviour (e.g., computer use). \\
\hline & Anxiety & $\begin{array}{l}\text { Evoking anxious or emotional reactions when it comes to performing a } \\
\text { behaviour (e.g., using a computer). }\end{array}$ \\
\hline
\end{tabular}




\begin{tabular}{|c|c|c|}
\hline \multicolumn{3}{|l|}{ Models and Theories of Individual Acceptance } \\
\hline Theory of Reasoned Action (TA) & Core Constructs & Definitions \\
\hline \multicolumn{3}{|l|}{$\begin{array}{l}\text { The Unified Theory of Acceptance and Use of Technology } \\
\text { (UTAUT) }\end{array}$} \\
\hline \multirow{4}{*}{$\begin{array}{l}\text { The Unified Theory of Acceptance and Use of Technology } \\
\text { (UTAUT) by Venkatesh, et al. (2003) advances individual } \\
\text { technology acceptance research by unifying the many } \\
\text { theoretical perspectives common commonly used in the study } \\
\text { of IT in organisations. Out of the seven constructs that } \\
\text { appeared to be significant direct determinants of intention or } \\
\text { usage in one or more of the individual models, they theorized } \\
\text { that four constructs played significant role as direct } \\
\text { determinants of user acceptance and usage behaviour - } \\
\text { performance expectancy, effort expectancy, social influence } \\
\text { and facilitating conditions. Attitude toward using technology, } \\
\text { self-efficacy and anxiety were theorized not to be direct } \\
\text { determinants of intention. He incorporates four moderators } \\
\text { (gender, age, experience, voluntariness of use) to account for } \\
\text { dynamic influences including organisational context, user } \\
\text { experience and demographic characteristics. He examines the } \\
\text { effect of these determinants on intention to facilitate fair } \\
\text { comparison of the different models (Venkatesh, Morris et al. } \\
\text { 2003). }\end{array}$} & Performance expectancy & $\begin{array}{l}\text { "the degree to which an individual believes that using the system will help } \\
\text { him or her to attain gains in job performance" (Venkatesh, Morris et al. } \\
\text { 2003, p.447). The five constructs from the different models that pertain to } \\
\text { performance expectancy are perceived usefulness (TAM/TAM2 and } \\
\text { C_TAM_TPB), extrinsic motivation (MM), job-fit (MPCU), relative } \\
\text { advantage (DOI) and outcome expectations (SCT). }\end{array}$ \\
\hline & Effort expectancy & $\begin{array}{l}\text { "the degree of ease associated with the use of the system" (Venkatesh, } \\
\text { Morris et al. 2003, p.450). The concept of effort expectancy is captured in } \\
\text { three constructs from the existing models: perceived ease of use } \\
\text { (TAM/TAM2), complexity (MPCU) and ease of use (DOI). }\end{array}$ \\
\hline & Social influence & $\begin{array}{l}\text { "the degree to which an individual perceives that important others believe } \\
\text { he or she should use the new system (Venkatesh, Morris et al. 2003, } \\
\text { p.451). Social influence as a direct determinant of behavioural intention is } \\
\text { represented as subjective norm in TRA, TAM2, TPB/DTPB and C-TAM- } \\
\text { TPB, social factors in MPCU and image in DOI. Each of these constructs } \\
\text { contains the explicit or implicit notion that the individual's behaviour is } \\
\text { influenced by the way in which they believe others will view them as a } \\
\text { result of having used the technology (Venkatesh, Morris et al. 2003). }\end{array}$ \\
\hline & Facilitating conditions & $\begin{array}{l}\text { "the degree to which an individual believes that an organizational and } \\
\text { technical infrastructure exists to support use of the system” (Venkatesh, } \\
\text { Morris et al. 2003, p.453). This definition captures concepts embedded by } \\
\text { three different constructs: perceived behavioural control (TPB/DTPB, C- } \\
\text { TAM-TPB), facilitating conditions (MPCU) and compatibility (DOI). Each } \\
\text { of the constructs is set to include aspects of the technological and/or } \\
\text { organisational environment that are designed to remove barriers to use } \\
\text { (Venkatesh, Morris et al. 2003). }\end{array}$ \\
\hline
\end{tabular}




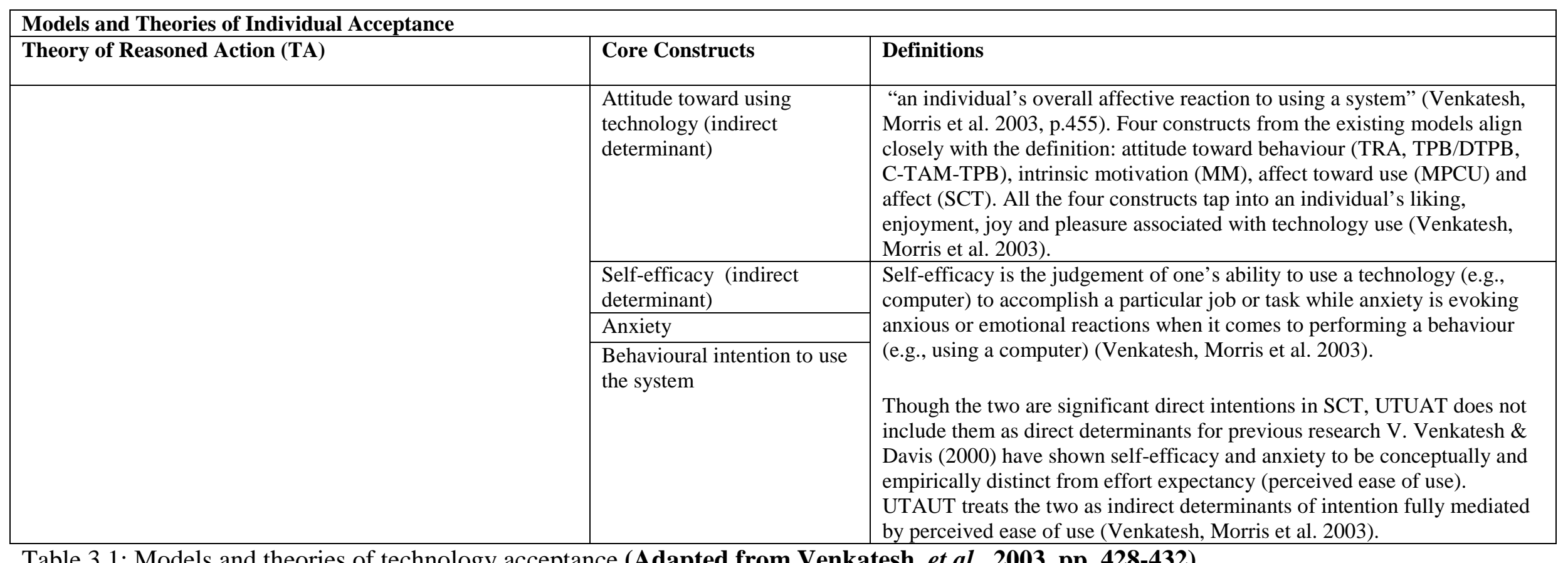

Table 3.1: Models and theories of technology acceptance (Adapted from Venkatesh, et al., 2003, pp. 428-432) 
This study was particularly informed by Rogers’ Diffusion of Innovations (DOI) and Hofstede's Cultural Dimensions theories, which are discussed in detail in the following subsections. The two models complement each other in studies of innovations diffusion, adoption and use.

\subsubsection{Rogers' Diffusion of Innovations theory (DOI)}

The DOI derives from Rogers’ studies spanning over four decades (1962, 1971, 1983, 1995 and 2003). It gives a theoretical basis for conceptualising the introduction and adoption of new innovations for it has a strong application focus and is tied to empirical generalisations (Tetiwat 2003; Tran 2006; Bagchi and Udo 2007). Its usefulness and popularity stems from its easy application (Clarke 1999; Tran 2006).

Adoption is a key factor in the diffusion process. Lee et. al. (1999) define adoption as the process through which an organisation decides to acquire systems or technology. Diffusion is "the process in which an innovation is communicated through certain channels over time among the members of a social system” (Rogers 2003, pp.5,11). Thus, four main elements characterise the diffusion of innovations process: innovation, communication channels, time, and the social system. Each element is crucial because it affects the rate of adoption of innovations. The diffusion of innovations explains social change and is, therefore, an essentially social process; for it to become self-sustaining, the rate of adoption of the innovation should reach critical mass (Rogers 2003, pp.viii,xx,5).

Rogers (2003) defines innovation as "an idea, practice, or object that is perceived as new by an individual or other unit of adoption" (p. 12). Innovation diffusion is "a special type of communication in which the messages are about a new idea” (p.6). The newness denotes some degree of uncertainty that is overcome by seeking information. Innovation is at times used synonymously with technology, which Rogers defines as "a design for instrumental action that reduces the uncertainty in the cause-effect relationships involved in achieving the desired outcome” (Rogers 2003, p.13). Rogers sees innovation as a means of reducing uncertainty and this is made possible by information about the cause-effect relationships on which the technology is based. He argues that, even though a technological innovation should have at least some degree of benefits for its potential adopters, they are seldom certain that an 
innovation represents a superior alternative to the previous practice that it would replace, at least when they initially learn about it. Thus, knowing about a technological innovation creates uncertainty about its consequences (effects) in the mind of potential adopters (p. 14).

Diffusion research focuses on how technological (or other) innovations are implemented (or not) in social systems, including organisations. At the heart of diffusion research are the innovation-decision process and the conditions for a successful innovation process. Hence, this stream of research contributes to an explanation of the status quo in any given situation of a diffusion process. The main focus is on individual actors (users), and their behaviour and attitudes. This is important because technologies are co-shaped by "the social", the institutional framework, and the various actors; technology is conceptualised as nondeterministic in that its effects cannot be explained solely by looking at the technology itself (Nentwich 2003, pp.26-28).

Rogers (2003) sees the innovation-decision process as "essentially an information-seeking and information-processing activity in which an individual is motivated to reduce uncertainty about the advantages and disadvantages of the innovation” (p. 14). It is "the process through which an individual (or other decision-making unit) passes from first knowledge of an innovation, to forming an attitude toward the innovation, to a decision to adopt or reject, to implementation of the new idea, and to confirmation of this decision” (p. 216). It is a five stage process:

1) Knowledge, when the individual is exposed to the innovation's existence and gains an understanding of how it functions;

2) Persuasion, when the individual forms a favourable or unfavourable attitude towards the innovation;

3) Decision, when the individual engages in activities that lead to a choice to adopt or reject the innovation;

4) Implementation, when the individual puts an innovation into use; and 
5) Confirmation, when the individual seeks reinforcement for an innovation-decision already made but may reverse the decision if exposed to conflicting messages about it (Rogers 2003).

The argument advanced in DOI tallies with the object of this research study, which set out to investigate the socio-economic, cultural, institutional and technological factors affecting the diffusion, adoption and use of a new innovation, ICT, in research communication among researchers in Kenya research institutions. Diffusion is a kind of social change that is expected to bring about alterations in the structure and function of a social system (Rogers, 1995, 2003; Rogers \& Shoemaker, 1971; Rogers, 1976; Van de Ven \& Rogers, 1988). The proposed study is motivated by the need for a social change in research communication in Kenya, necessitated by the invisibility and inaccessibility of the Kenyan research output, despite many propositions that modern ICT, especially the Internet, are a solution to the research communication crisis. Rogers (2003) says "the Internet can largely remove the cost of communicating across space" and that "diffusion via the Internet greatly speeds up an innovation's rate of adoption” (p.216). The study, therefore, seeks to use Rogers’ DOI model with a view to coming up with a contextual framework to bring about the diffusion, adoption and use of new ICT in communicating research output among researchers in research institutions in Kenya.

\section{1.1.1 Variables determining the rate of adoption of innovations}

Rogers (2003) outlines five variables that affect an innovation's rate of adoption as: perceived attributes of an innovation; the type of innovation-decision; the nature of communication channels diffusing the innovation at various states in the innovation-decision process; the nature of the social system in which the innovation is diffusing; and the extent of change agents' promotion efforts in diffusing the innovation (see figure 3.1 below). 
I. Perceived Attributes of Innovations

1. Relative advantage

2. Compatibility

3. Complexity

4. Trialability

5. Observability

II. Type of Innovation-Decision

1. Optional

2. Collective

3. Authority

III. Communication Channels (e.g. mass media or interpersonal)

IV. Nature of the Social System (e.g., norms, degree of network interconnectedness, etc.)

V. Extent of Change Agents’ Promotion Efforts

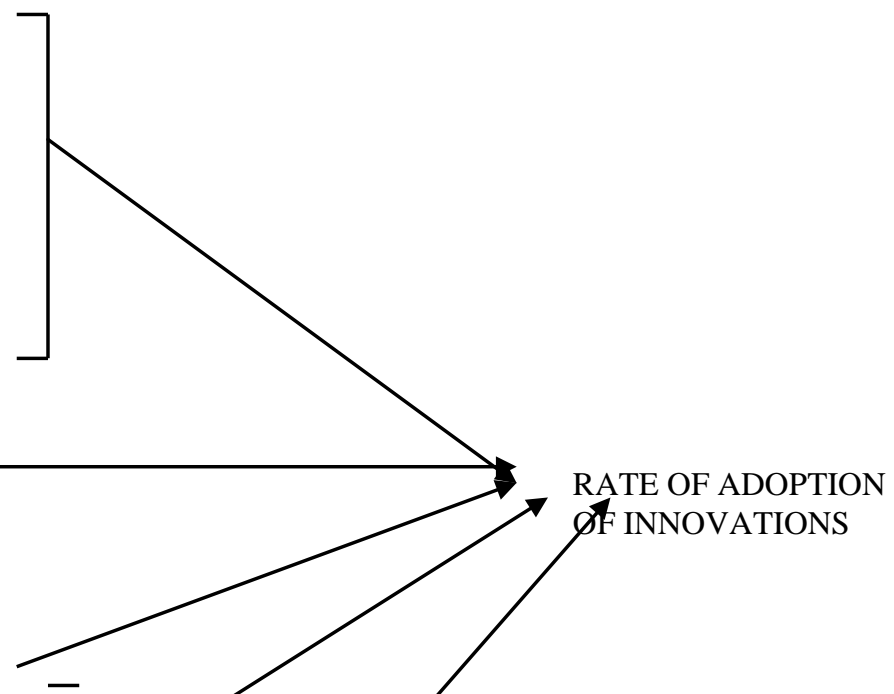

Figure 3.1: Variables determining the rate of adoption of innovations (Rogers 2003)

\section{Perceived Attributes of Innovations}

Rogers (2003. pp.223-266) gives five characteristics of innovations which affect the rate of adoption:

- Relative advantage: the degree to which an innovation is perceived as being better than the idea it supersedes (e.g., economic profitability, low initial cost, a decrease in discomfort, social prestige, a saving of time and effort, and immediacy of reward).

- Compatibility: the degree to which an innovation is perceived as consistent with the existing values, past experiences, and needs of potential adopters (e.g. socio-cultural values and beliefs, previously introduced ideas, and/or client needs for the innovation).

- Complexity: the degree to which an innovation is perceived as relatively difficult to understand and use. 
- Trialability: the degree to which an innovation may be experimented with on a limited basis.

- Observability: the degree to which the results of an innovation are visible to others.

\section{Type of Innovation-Decision}

There are three types of innovation-decisions:

- Optional innovation-decisions: choices to adopt or reject an innovation that are made by an individual independent of the decisions by other members of a system.

- Collective innovation-decisions: choices to adopt or reject an innovation that are made by consensus among the members of a system.

- Authority innovation-decisions: choices to adopt or reject an innovation that are made by a relatively few individuals in a system who possess power, high social status, or technical expertise.

The more persons involved in making an innovation-decision, the slower the rate of adoption (Rogers 2003). Innovations requiring an individual-optional innovation-decision are generally adopted more rapidly than when an innovation is adopted by an organisation. The type of innovation-decisions has a bearing on leadership: the people who make decisions on implementation of innovations, whose role in innovation adoption is determinant (Damanpour \& Schneider, 2006, 2009; Elenkov \& Manev, 2005; Moon, 2000; Moon \& Bretschneider, 2002; Nystrom, Ramamurthy \& Alla, 2002; Thong \& Yap, 1995).

\section{Communication channels}

These are means by which a message gets from the source to the receiver. Communication channels are categorised into: (1) interpersonal or mass media in nature and (2) as originating from either localite or cosmopolite sources. Mass media channels transmit messages that involve a mass medium, e.g. radio, television, newspapers, etc., that enables a source of one or a few individuals to reach an audience of many. Interpersonal channels involve a face-toface exchange between two or more individuals (Rogers 2003). 
Mass media channels are relatively more important at the knowledge stage, and interpersonal channels are relatively more important at the persuasion stage in the innovation-decision process. Mass media channels are relatively more important than interpersonal channels for earlier adopters than for later adopters (Rogers, 2003). Cosmopolite channels are relatively more important at the knowledge stage, and localite channels are relatively more important at the persuasion stage in the innovation-decision process. Nevertheless, Reardon and Rogers (1988) argue there is no theoretical justification for separation of interpersonal and mass media channels.

\section{Nature of Social System}

A social system is a set of interrelated units engaged in joint problem solving to accomplish a common goal. The social and communication structure of a system can facilitate or impede the diffusion of innovations in the system, for example, norms ${ }^{2}$ and the degree of network interconnectedness ${ }^{3}$. In this study, the social system is the research community.

\section{Extent of change agents' promotion efforts}

Change agents are individuals within organisations who work to promote a specific innovation or kind of behaviour. Though the relationship between the rate of adoption and change agents' efforts may not be direct or linear, Rogers explains that a greater payoff from a given amount of change agent activity with the assistance of opinion leaders occurs at certain stages in an innovation's diffusion until a critical mass of adopters is reached (Rogers, 2003, pp.222-223). In this study, the research institution, individual researchers, librarians and journal editors who have adopted ICT for research communication are regarded as the change agents, while ICT adoption and use are the focus. One of the issues to be investigated is to what extent researchers are change agents.

\footnotetext{
${ }^{2}$ The established behaviour patterns for the members of a social system.

${ }^{3}$ A communication network of interconnected individuals who are linked by patterned flows of information, e.g. opinion leaders, change agents

Rogers, E. M. (2003). Diffusion of innovations. New York, Free press.
} 


\subsubsection{Use of DOI in information studies research and justification for use in this study}

Rogers' DOI has been chosen for this study because of its wide use in many disciplines and contexts (See summary of studies that have used the DOI in appendix A-4).

The researcher chose to be guided by the DOI theory because it has been constantly revised to accommodate changing times and scholarly discourse over the last 44 years and widely used in many different cultural backgrounds (Rogers, 1995, 2003, 2004). Further, as Clarke (1999) further explains, “The theory has potential application to information technology ideas, artefacts and techniques, and has been used as the theoretical basis for a number of IS research projects. ... it provides one valuable 'hook' on which research and practice can be hung,” (n.p). Thus, the theory has withstood the test of time and continues being referred to and used by researchers and scholars across cultures in discussions on diffusion, acceptance and adoption of technology and other innovations.

Moreover, compared to other innovations diffusion models like TAM (Technology Acceptance Model) which focuses on perceived usefulness (Rogers' relative advantage) and perceived ease of use (Rogers' complexity), DOI is more encompassing in that it addresses a wider range of variables (Bagchi \& Udo, 2007; Tran, 2006; Venkatesh, et al., 2003) and thus provides for a more comprehensive analysis of innovation diffusion (Tetiwat 2003). For example, in a study undertaken by Plouffe et al. (2001) comparing the models in terms of subjects' behavioural intention to adopt and use technology in the context of a market trial of an electronic payment system using smart cards, the variance in intention explained by TAM was 33\% while DOI was 45\% (see also Venkatesh et al. (2003, p.436). Other models like TRA (Theory of Reasoned Action) and TPB (Theory of Planned Behaviour), among others, focus on behavioural aspects, which is not wide enough for what the scope of this study envisages.

DOI has also been used in many technology diffusion studies that have identified gaps pertaining to the need to undertake research on the socio-economic, cultural, technological and institutional factors affecting the diffusion, adoption and use of ICT by different user groups. Rogers, Takegami and Yin (2001) say the process of technology transfer is a difficult type of communication, which demands trained and skilled personnel, adequate resources, 
and organisational and other incentive structures. Van de Ven and Rogers (1988) are critical of past research on innovations in organisations and call for a focus on process research in future investigations. A study by Hargittai (2001) suggests digital inequality be considered at five different levels, namely differences in technical apparatus used to access the Internet, location of access (autonomy of use), the extent of users' social support networks, the types of use to which the medium is put, and users' level of skill.

Findings of a study by Lal (2001) recommended that the institutional environment plays a crucial role in the diffusion and production of ICT in developing countries. Another study by Oyelaran-Oyeyinka and Lal (2005) recommended further research examine the role of institutional infrastructure in the diffusion of ICT in general, and the Internet in particular. Oyelaran-Oyeyinka and Adeya (2002), in a study on Internet diffusion in Nigerian universities, conclude that ease of access is a significant factor in the Internet's diffusion. Oyelaran-Oyeyinka and Lal (2005) argue, "In as much as the diffusion of technologies is dynamic, it is the changes in the configuration of technologies and the social use to which they are put that over time may well prove the most challenging for theory and policy" (p.509). Other recent ICT adoption and diffusion studies have also emphasised the need for an understanding of the socio-cultural, institutional and technological environments (Bagchi \& Udo, 2007; Bakkabulindi, Nkata \& Amin, 2008; Damanpour \& Schneider, 2006, 2009; Keengwe, Kidd, \& Kyei-Blankson, 2009; Moon \& Bretschneider, 2002; Odero-Musakali \& Mutula, 2007). Thus, ICT diffusion and adoption are very much tied to the social and the technological attributes of the technology itself, and therefore the need for context-specific investigations for effective ICT adoption frameworks and strategies.

Despite the comprehensiveness of Rogers' DOI, it has been criticised for excluding "the possibility of influence from organizational and environmental factors” (Lee \& Cheung, 2004, p.386). Yet in line with the MIS model ( Ives, Olson, \& Baroudi, 1993), research in Information Systems (IS) adoption should incorporate the views from users, organisation, and external environment. Bagchi (2001) says DOI models “do not address potential national sources of influence” (p. 35) and argues that "organizations may have branches in many nations staffed by individuals and circumstances whose sources of influence to adopt IT are indigenous. Even if organisations operate within the boundary of a single-nation, they are 
dependent on that nation's characteristics. National-level characteristics can form a distinct set of separate factors that play a significant role in the adoption and diffusion of IT" (Bagchi 2001, p. 35). Though he acknowledges the ability of DOI to provide “one valuable 'hook' on which research and practice can be hung”, Clarke (1999) criticises the theory as weak in its explanatory power and usefulness in predicting outcomes outside North America from whose culture DOI is derived.

Nevertheless, its versatile application in studies across cultures over time is an indication of the robustness of the DOI theory. This motivated the researcher to use the theory in this interpretive study as a lens to guide exploration of factors affecting ICT-mediated research communication, while taking care to avoid some of the pitfalls associated with the theory, such as neglecting influence from organisational and national environment.

Rai, Ravichandran and Samaddar (1998) hold that temporal internal diffusion models cannot adequately explain the diffusion process of the Internet. They suggest a mixed influence model that combines internal and external influences would be better suited to model the diffusion process of the Internet. Others who have challenged the diffusion theory include Abrahamson (1991), whose work provides an alternate explanation as to why some diffusions succeed and others fail. His model accounts for the fact that the mere acquisition of an IT product may not mean its adoption by a firm or subsequent diffusion of the product in the industry (Bagchi 2001). Likewise, Fichman and Kemerer (1999) highlight an assimilation gap between the acquisition and development of IT products like Computer-Aided Software Engineering (CASE). Bagchi (2001) criticises the assumption by the internal model that diffusion occurs only through interpersonal contact and at the same time points that "the external model assumes no contact between prior and present adopters and that adequate information is available through sources external to the system” (p.43). The internal model is a type of diffusion process where information and influence flow within the adopting population whereas within the external model information and influence flow into a population (Strang \& Soule, 1998). Also, the diffusion research has been criticised for its proinnovation bias, individual-blame bias, recall problems regarding accuracy of incidents that serve as diffusion data, and issues of equality (Rogers, 1995; Bagchi, 2001). 
To counter the criticisms above, this study combined DOI with Hofstede's cultural dimensions (Hofstede, 2001, 1991, 1983, 1980), thereby broadening the base on which factors of the national culture environment affecting the diffusion, adoption and use of ICT in the research communication process among researchers in Kenya could be investigated. Moreover, the criticisms above made the researcher aware that the guiding theories were not foolproof; so, the researcher was alert throughout and sufficiently flexible to note nuances outside the confines of the theories.

\subsubsection{Hofstede's Cultural Dimensions model}

Hofstede is one of the foundational theorists in the field of intercultural communication. He defines national culture as the collective programming of the mind that differentiates members of one group from other groups and says that culture is learnt and cannot be inherited. It calls for compliance with certain values, rules and practices in a society and consequently, influences the ability of humans to feel, learn and communicate (Hofstede 1980; Hofstede 1980; Hofstede 2001).

The Cultural Dimensions theory is the product of Hofstede's research on national culture focusing on IBM employees from over 70 countries around the world and with a sample size of over hundred thousand (Hofstede 2001). The findings of the study, which is identified as a major breakthrough in social sciences, identified five dimensions of national culture which represent the mainstream attitude, expectations, values and behaviours. These include:

1) Power distance (categorised as either large or small): This measure refers to the extent to which a society accepts the unequal power distribution within or between institutions and firms. It is related to the different solutions to the basic problem of human inequality or differences in power and wealth by different societies.

Large power distance (PD) means there is a greater difference in distribution of wealth and power among members of the society. People in large power distance societies are more comfortable with a larger status differential than those in small power distance societies.

Small power distance means that there is not much difference in distribution of wealth and power among members of society. 
2) Uncertainty avoidance (categorised as strong/high or weak/low) relates to the level of stress in a society in the face of unknown future. This cultural attribute describes the extent to which individuals feel threatened by uncertain and ambiguous situations, and try to avoid them. People in countries that have low uncertainty avoidance (UA) feel much more comfortable with the unknown, whereas extreme uncertainty creates intolerable anxiety of an uncertain future among people with high uncertainty avoidance. In cultures with high/strong uncertainty avoidance, mechanisms are created to provide reinforcement and reduce risks. Thus, institutions in such cultures may have more rigid rules and exhibit less tolerance for uncommon ideas and behaviours.

In countries with strong uncertainty avoidance, people tend to be active, aggressive, emotional, compulsive, security-seeking, and intolerant whereas people in weak uncertainty avoidance countries tend to be easy-going, indolent, controlled, less aggressive, unemotional, relaxed, accepting of personal risks, and relatively tolerant.

3) Individualism versus collectivism (categorised as high or low) relates to the integration of individuals into primary groups.

In countries with high individualism, the relationships between individuals are loose and people are expected to look after themselves and their immediate families. In low individualism (or collectivism) (IC) countries, the relationships between people in the same family mean that these people are integrated into strong and cohesive groups that last a lifetime. Family members protect each other in exchange for unquestioning loyalty.

4) Masculinity versus femininity (categorised as strong or weak) relates to the division of emotional roles between men and women and the expected gender roles in a society. Strong masculinity cultures have very distinct expectations of men and women in the society. Men tend to be assertive, ambitious, tough, competitive, and focus on material success, while women tend to be tender, more modest, and concerned with quality of life. Thus, to the extent that a culture is feminine, the values of human relationships and concern for others are high, while, on the other hand, masculine cultures are more assertive and value materialism. Strong masculinity cultures are, therefore, associated with competitiveness and aggression when it comes to the adoption of new innovations. Conversely, weak masculinity (femininity) (M/F) cultures have a greater vagueness about what is expected of each gender. Men and women 
tend to be equally modest and tender and concerned with quality of life. A culture of cooperation/collectiveness prevails in such cultures.

5) Long-term versus short-term orientation (Confucian dynamism) relates to the choice of focus for people's efforts: the future or the present. This dimension was added later and focuses on the differences between East Asian countries and the rest of the world. It was derived from the teachings of Confucius and accounts for the relationship between an individual's work ethic and his/her respect for tradition. Cultures with high Confucian dynamism value persistence and perseverance, status-oriented relationships, thrift and a conscious sense of shame. They attribute more importance to respect for tradition, protecting one's face and the reciprocation of gifts, greetings or favours than personal steadiness and stability (Hofstede 2001).

Out of the over 70 countries in Hofstede's study, 53 countries were rated and their performance indices calculated based on the five cultural dimensions. The research findings classified East Africa (encompassing Kenya) as belonging to the dimensions of large power distance (64); strong uncertainty avoidance (52); low individualism (27) and weak-to- average masculinity (41). The African countries that were tested on long-term versus short-term orientation were ranked as having a weak index (25 for Zimbabwe and 16 for Nigeria) (See the table 3.2 below). This group is said to present a fairly risk-averse market, and members of such societies are said to follow those in charge with little desire to participate or direct.

On the other hand, Hofstede and studies using Hofstede's paradigm have shown countries with a small power distance, high level of individualism, a strong level of masculinity and a low level of uncertainty avoidance as generally the most innovative group with relatively weak resistance to new products and a strong desire for novelty and variety (Bagchi, 2001; Hofstede, 2001,1991, 1983, 1980; Keil, Tan, Wei, Saarinen, Tuuainen, \& Wasenaar, 2000; Tetiwat, 2003; Tan, Wei, Watson \& Walczuch, 1998; Hill, et al., 1998). This can be seen in the low adoption of web-based technologies in Thailand as opposed to New Zealand (Tetiwat, 2003); the low adoption of the Internet in Arab countries and the slow adoption of email use as opposed to FAX in Japan, unlike in America (Straub, 1994). Hence, when it comes to adoption of new technologies, institutions from regions like Africa may also tend to wait until 
others have adopted, leading to low adoption levels for some of them.

Table 3.2: Hofstede’s Dimension of Culture Scales

\begin{tabular}{|l|c|c|c|c|c|}
\hline Country & $\begin{array}{l}\text { Power } \\
\text { Distance }\end{array}$ & Individualism & $\begin{array}{l}\text { Uncertainty } \\
\text { Avoidance }\end{array}$ & Masculinity & $\begin{array}{l}\text { Long term } \\
\text { orientation }\end{array}$ \\
\hline $\begin{array}{l}\text { Arab } \\
\text { countries }\end{array}$ & 80 & 38 & 68 & 53 & \\
\hline Argentina & 49 & 46 & 86 & 56 & \\
\hline Australia & 36 & 90 & 51 & 61 & 31 \\
\hline Austria & 11 & 55 & 70 & 79 & \\
\hline Belgium & 65 & 75 & 94 & 54 & 65 \\
\hline Brazil & 69 & 38 & 76 & 49 & 23 \\
\hline Canada & 39 & 80 & 48 & 52 & 118 \\
\hline Chile & 63 & 23 & 86 & 28 & \\
\hline $\begin{array}{l}\text { China, } \\
\text { Mainland }\end{array}$ & & & & & \\
\hline Colombia & 67 & 13 & 80 & 64 & \\
\hline $\begin{array}{l}\text { Costa } \\
\text { Rica }\end{array}$ & 35 & 15 & 86 & 21 & \\
\hline
\end{tabular}

Table 3.2: Hofstede’s Dimension of Culture Scales

\begin{tabular}{|l|c|c|c|c|c|}
\hline Country & $\begin{array}{l}\text { Power } \\
\text { Distance }\end{array}$ & Individualism & $\begin{array}{l}\text { Uncertainty } \\
\text { Avoidance }\end{array}$ & Masculinity & $\begin{array}{c}\text { Long term } \\
\text { orientation }\end{array}$ \\
\hline Denmark & 18 & 74 & 23 & 16 & \\
\hline East Africa & 64 & 27 & 52 & 41 & \\
\hline Equador & 78 & 8 & 67 & 63 & \\
\hline Finland & 33 & 63 & 59 & 26 & \\
\hline France & 68 & 71 & 86 & 43 & \\
\hline $\begin{array}{l}\text { Germany } \\
\text { FR }\end{array}$ & 35 & 67 & 65 & 66 & 31 \\
\hline $\begin{array}{l}\text { Great } \\
\text { Britain }\end{array}$ & 35 & 89 & 35 & 66 & 25 \\
\hline Greece & 60 & 35 & 112 & 57 & \\
\hline Guatemala & 95 & 6 & 101 & 37 & \\
\hline Hong Kong & 68 & 25 & 29 & 57 & 96 \\
\hline India & 77 & 48 & 40 & 56 & 61 \\
\hline Indonesia & 78 & 14 & 48 & 46 & \\
\hline Iran & 58 & 41 & 59 & 43 & \\
\hline Ireland & 28 & 70 & 35 & 68 & \\
\hline
\end{tabular}




\begin{tabular}{|l|c|c|c|c|c|}
\hline Country & $\begin{array}{l}\text { Power } \\
\text { Distance }\end{array}$ & Individualism & $\begin{array}{l}\text { Uncertainty } \\
\text { Avoidance }\end{array}$ & Masculinity & $\begin{array}{l}\text { Long term } \\
\text { orientation }\end{array}$ \\
\hline Israel & 13 & 54 & 81 & 47 & \\
\hline Italy & 50 & 76 & 75 & 70 & \\
\hline Jamaica & 45 & 39 & 13 & 68 & \\
\hline Japan & 54 & 46 & 92 & 95 & 80 \\
\hline Malaysia & 104 & 26 & 36 & 50 & \\
\hline Mexico & 81 & 30 & 82 & 69 & \\
\hline Netherlands & 38 & 80 & 53 & 14 & 44 \\
\hline $\begin{array}{l}\text { New } \\
\text { Zealand }\end{array}$ & 22 & 79 & 49 & 58 & 30 \\
\hline Norway & 31 & 69 & 50 & 8 & \\
\hline Pakistan & 55 & 14 & 70 & 50 & \\
\hline Panama & 95 & 11 & 86 & 44 & \\
\hline Peru & 64 & 16 & 87 & 42 & \\
\hline Philippines & 94 & 32 & 44 & 64 & 19 \\
\hline Poland & & & & & 32 \\
\hline Portugal & 63 & 27 & 104 & 31 & \\
\hline Salvador & 66 & 19 & 94 & 40 & \\
\hline Singapore & 74 & 20 & 8 & 48 & 48 \\
\hline $\begin{array}{l}\text { South } \\
\text { Africa }\end{array}$ & 49 & 65 & 49 & 63 & \\
\hline $\begin{array}{l}\text { South } \\
\text { Korea }\end{array}$ & 60 & 18 & 85 & 39 & 75 \\
\hline Spain & 57 & 51 & 86 & 42 & \\
\hline Sweden & 31 & 71 & 29 & 5 & 33 \\
\hline Switzerland & 34 & 68 & 58 & 70 & \\
\hline Taiwan & 58 & 17 & 69 & 45 & \\
\hline Thailand & 64 & 20 & 64 & 34 & 56 \\
\hline Turkey & 66 & 37 & 85 & 45 & \\
\hline Uruguay & 61 & 36 & 100 & 38 & \\
\hline USA & 40 & 91 & 46 & 62 & 29 \\
\hline Venezuela & 81 & 12 & 76 & 73 & \\
\hline $\begin{array}{l}\text { West } \\
\text { Africa }\end{array}$ & 77 & 20 & 54 & 46 & \\
\hline Yugoslavia & 76 & 27 & 88 & 21 & \\
\hline & & & & & \\
\hline
\end{tabular}

Critics of Hofstede have argued that his view on national culture as a uniform and unchanging entity is weak, narrow, simplistic and misleading (Ali \& Brooks, 2009; McCoy, 2003; McSweeney, 2002; Myers \& Tan, 2002; Redding, 1994; Smith, 1998; Sondergaard, 1994; 
Tayeb, 1994; Winch, et al. 1997; Yeh \& Lawrence, 1995). Sondergaard (1994) sees Hofstede's use of aggregated personal traits to characterise a particular culture as an oversimplification because different subcultures are not represented in the aggregation. Moreover, he argues that the matching of cultures and management styles is not systematic. He further says that Hofstede does not represent changes in values over time; for example, a study by Fernandez, Carlson, Stepina \& Nicholson, 1997 (cited in Bagchi, 2001, p. 48) found that there had been a shift in values for nine of the nations since Hofstede's main study in 1980). Myers and Tan (2002) argue that the concept of national culture is theoretically weak and ignores some of the facts of history and has little explanatory power. They hold the notion of nation - state is a relatively recent phenomenon compared to the long existence of culture and there is no necessary alignment between a nation-state which a political entity and culture. They call upon IS researchers to go beyond the national culture concept to view culture as emergent, contested and divergent in different contexts. Tayeb (1994) concurs and stresses the need to employ multiple methodologies in studies on culture.

The researcher took cognisance of the criticisms and used a combination of methodologies as suggested by Tayeb (1994) to bring out the various perceptions regarding ICT adoption to ICT by researchers within their own cultural contexts Moreover, the researcher was encouraged by the fact that, despite the criticisms, Hofstede's research has been widely cited and used in many studies, both qualitative and quantitative, over a long time and has provided both a theoretical paradigm and data used in other studies (Sondergaard, 1994; Myers \& Tan, 2002; Tayeb, 1994). Others have also replicated his work, like Hoppe (1990) who provided validation for Hofstede’s observations.

\subsubsection{Use of Hofstede’s Cultural Dimensions model in the literature}

Hofstede's theory has been used in diffusion studies in many disciplines - anthropology, psychology, history, sociology, law, economics, business administration and information science (Hofstede, 2001). For example, Nakata and Shivakumar (1996) used Hofstede’s dimensions to provide a theoretical framework for a product development. Dwyer, Mesak and Hsu (2005) undertook a study linking Hofstede’s dimensions to the diffusion rates of five consumer products, which found a strong relationship between Hofstede's dimensions and the 
diffusion rates of different products. Another study by Steenkamp, Hofstede and Weddle (1999), found that three national cultural dimensions of Hofstede (IC, UA and MF) were influential in consumer innovativeness.

Other studies on culture have included Schwarz et al.(1990; 1995) who identified ten cultural values: self-direction, hedonism, tradition, power, stimulation, universalism, benevolence, conformity, security, and achievement. These can be organised in a motivational continuum in orthogonal dimensions - openness to change versus conservation, and self-transcendence versus self-enhancement (Schwarz and Sagiv, 1995) - that could be related to Hofstede's power distance dimension. Smith, Dugan and Trompenaars' (1996) in a study focusing on three dimensions, universalism/particularism, achievement/ascription and individualism/ collectivism, found that Hofstede's dimensions like individualism/collectivism are better defined in terms of group membership (loyal to group or utilitarian). They found that power distance could be better defined in terms of obligation to social relationship (conservatism versus egalitarian). Later, Trompenaars and Hampden-Turner (1993) categorised three cultural dimensions: relationships with people, passage of time and relationship with environment. Thus, Hofstede's study seems to be more elaborate and encompassing than other studies on culture, and therefore, the reason the researcher chose it to guide this research.

\subsubsection{Use of Hofstede's Cultural Dimensions model in information research}

In his study on impacts of national culture and other national level factors on adoption of IT, Bagchi makes the conclusion that, "As long as cultural differences persist and nations look similarly dissimilar, cultural values will continue to influence the way IT is adopted and diffused in different nations” (Bagchi, 2001, p.332). This shows the importance of addressing the cultural dimension of ICT adoption in different cultures. He argues that, "If IT changes follow certain paths contingent on the cultural background of a society, those who are aware of the cultural background will be better prepared to understand the future development of ITs in that nation” (Bagchi, 2001, p.329). He echoes the same in a later study (Bagchi and Udo, 2007). However, many studies on adoption of IT/ICT have failed to address this critical dimension. It is for this reason that this study, using DOI and Hofstede's Cultural Dimension theories perspectives, investigated the social, cultural, institutional and technological factors affecting adoption of ICT for research communication in research institutions in Kenya. 
Mere technological superiority of an IT product may not make it successful in every nation, because the attitudes and behaviours of the people using it, which are critical to its success, may vary widely from one nation to another (Bagchi, 2001). Child (1980, cited in Bagchi (2001)) found that even though organisations may look similar in structure, employees inside an organisation maintain their unique cultural practices. Hofstede (1980; 2001) found that national culture explained $50 \%$ of the variance in employees' attitudes and behaviours at IBM, a multinational company.

Many IS researchers have used Hoefstede's theory in studies on IT in different cultural settings. In a study incorporating the five cultural dimensions, Herbig and Muller (1992) described how outsourcing and adoption depend on cultural attributes, which led them to conclude that "technology follows culture" (p. 75) Straub (1994), in a comparative study focusing on the role of culture in the adoption of email and FAX in Japan and the US, found that Hofstede's dimension of UA and the structural characteristics of the Japanese written language were related to Japanese knowledge workers' tendency to favour FAX over email systems. He recommended for sampling from many nations over time in IT diffusion studies. Hence, this study is an effort to fill such a gap.

A comparative study by Mejias (1995) investigating the adoption and use of Group Support Systems (GSS) between the US and Mexico found that Hofstede's dimensions explained some of the variance between the two countries. Also, in another study focusing on the relationship among cultural values, nationality, personal privacy and IT-related privacy regulations, Milberg, Burke, Smith and Kallman (1995) found that Hofstede's cultural dimensions explained variations in attitudes toward personal privacy. However, a case study of computer-related activities in south-western Nigeria found that socio-economic factors played a more major role in IT system development than traditional culture (Korpela, 1996) (See summary of selected references in appendix A-5) .

Many studies on IT-related diffusion have dealt more with task, individual and organisational related factors than the national level culture and other factors (Bagchi, 2001). For example, Kwon Zmud (1987) and Prescott and Conger (1995) have provided models of IT adoption but do not mention national indicators like culture. Nor do Fichman and Kemerer (1999) mention 
national culture interplay in their review of the extant literature on predictors of IT diffusion and assimilation. Yet there is a strong relationship between national culture and economic development as well as national culture and social and political systems (Inglehart, 1995, cited in Bagchi (2001, p.4). There is also a relationship between national culture, as well as other national-level factors and IT diffusion (Bagchi, 2001). Goodman (1991) says that "there are important historical, social, cultural and economic reasons for computing and telecommunication disparities (among nations) and, for better or worse, these differences make the world a more complicated and interesting place” (p.19). One example is the differences in the growth of computerisation in different national cultures, with some countries like the Asian Tigers (South Korea, Taiwan, Singapore and Hong Kong) witnessing rapid growth while others like Africa have had low growth. Another case is the uneven adoption of mobile telephony across nations, which is not necessarily related to economic factors, as noted in a report (Intekom, 2000, cited in Bagchi, 2001) that stated 75\% of people in Finland and Iceland used mobile telephones compared to about $40 \%$ in the US, despite the US economy being stronger than the Finnish at the time.

A number of researchers have shown the need to investigate national-level factors and the inter-relationships among these factors, for a detailed understanding of diffusion of innovations, especially IT innovations (Ali \& Brooks, 2008, 2009; Ali, Brooks \& Alshawi, 2008; Bagchi, 2001; Galivan \& Srite, 2005; Nath \& Murphy, 2004; Weisinger \& Trauth, 2003). Rogers (1995, 2003) observed that societal cultural norms, personal preferences and biases influence adoption decisions. Lefevre and Lefevre (1996) noted that culture has a definite influence on IT but its role remains under-investigated. Prescott and Conger (1995) stress the need to integrate national culture into the Diffusion of Innovation theory because of increasing globalization. In a study on an Internet diffusion framework, Press, Burkhart, Foster and Goodman (1998) noted that national culture, language, morality and religion were cited as important factors by governments advocating strict control over the Internet. Bagchi (2001) shares the same views and add traditional values and social hierarchy.

In Japan, for example, the growth of Internet has been constrained by collective agreements, hierarchical administrative structures and language (Barlow, 1992, cited in Bagchi (2001). Another example is the case of the economically wealthy Middle East Islamic nations which 
have lagged behind in IT infrastructure due, in part, to what Goodman and Green (1992) see as cultural differences, 'where personal contact is preferred to colder and more abstract long distance ties...Middle Easterners rely very heavily on face-to-face communications' (p.24; see also Bagchi, 2001, p.8). Inglehart (1995, cited in Bagchi, 2001) points that the orthodox Islamic nations have never really adopted IT which they associate with the Western lifestyle which is seen to be intrusive to traditional Islamic values. Although an exception is noted where in recent times the Internet is being used to access electronic repositories of religious texts and inscriptions and mobile telephones to view prayer times and Qiblah directions, research and development in IT have been largely ignored (Bagchi, 2001).

\subsubsection{Use of Hofstede's Cultural Dimensions theory in this study}

Hofstede's theory which calls for an understanding of the cultural context in diffusion and use of technology is relevant to this study on factors that affect the needs and priorities for ICTenabled research communication by Kenyan researchers in agriculture, biology/ biotechnology, environmental science and health sciences. As shown by the preceding discussion of Hofstede's cultural dimensions, in high power distance societies like Kenya, organisational structures are relatively more hierarchical and attach a lot of importance to status and status symbols. Management decisions will mostly be centralised and the role of the manager that of an expert. This affects ICT adoption and use in that new ICT could be introduced to serve as status symbol with use initially limited to a relatively few individuals at higher levels of the hierarchy. This, in turn, lowers the rate of diffusion in high power distance cultures (Bagchi, 2001). On the other hand, in a low power distance society, organisational structures are relatively flat and the issue of status symbol is relatively unimportant. Management decisions will be decentralised and more participative. Consequently, ICT are likely to be introduced at all levels and in larger numbers earlier on, which means the rate of ICT adoption is likely to be faster and higher in such societies.

In high uncertainty avoidance cultures, the need to avoid failure means willingness to take risks is reduced to a minimum. Since any new technology is associated with at least some initial uncertainty, ICT adoption might be slower, at least in the initial stages, in these societies. Over time, however, computing technology contributes to the automation of many 
tasks. This reduces the ambiguity in routine day-to-day tasks and the uncertainty caused by the ever-present possibility of human error. To the extent that certain ICT products can be used to control processes and support improvements in decision-making, they contribute to reducing uncertainty. Therefore, it could be expected that the diffusion of those products would be greater in cultures characterised by high uncertainty avoidance, like Kenya, once benefits become obvious. In contrast, in low uncertainty avoidance cultures, the rate of ICT diffusion will be faster initially.

In high individualistic societies, decision-making is personal rather than collectivistic. Reward systems are geared to individual achievements and based on merit. Organisational cultures in these environments encourage employees to be individualistic and look after themselves. To the extent that ICT products such as PCs and cellular technologies promote individual performance and fulfill obligations to self, it is expected that their diffusion would be greater in countries whose cultures were characterised by individualism compared to those characterised by collectivism, like Kenya. Moreover, since wealthier nations exhibit a high degree of individualism and consumers in these cultures have more buying power than poorer collectivist nations; this in turn could translate to higher rate of diffusion of ICT (Bagchi, 2001). Hence this dimension guides the researcher in exploring how the collectivist nature of the Kenyan society affects the adoption of ICT for research communication, for an understanding of such is important in developing a contextual framework.

In a highly feminine society, quality of life is considered more important. Since some of the recent ICT like mobile computers and telephones promote quality of life (flexibility in work, more time with family), they will be adopted more readily in a feminine society. Alternatively, in a highly masculine society, high ambitions and the need to excel are more pronounced. Assertiveness and acquisition of material things take precedence over concern for people and quality of life. Specific behaviour and products, such as ICT, that are supportive of achievement and ambition could also be adopted more rapidly in a highly masculine society. However, in a highly masculine society like Kenya, differences in sex roles are more pronounced and sex inequality is regarded as beneficial. Such practices imply about half of the population (female) in a highly masculine society will face inequality. 
Adoption of technologies such as ICT will be made more by males, with females having less access, which may imply less adoption of ICT in these societies. Since, in a feminine society, sex roles are not differentiated and inequality among sexes is not encouraged, some ICT adoptions will increase. An understanding of how the masculinity/femininity dimension affects ICT adoption for scientific research communication by researchers in Kenya highlights what needs addressing in developing an effective contextual framework for ICTenabled communication.

Societies with high Long-Term Orientation prefer a pragmatic, future-oriented perspective rather than a conventional, historic or short-term point of view. They desire acceptance of change rather than concern for stability. Since ICT are known to introduce changes in work and personal lives and also to change conventional lifestyles to more modern ones, the rate of ICT diffusion is expected to be higher in cultures that have high Long-Term Orientation in as long the ICT reinforces the status quo (Bagchi, 2001).

On the other hand, for instance, the human inequality or differences in power and wealth by different societies affect international knowledge communication or sharing, creating what Altbach calls "centres" and "peripheries" in his study of international knowledge distribution and Third World research publishing (Altbach 1977; 1978; 1981; 1982; 1987; 1992; 1993). Altbach uses the concept of "centres" and "peripheries" to characterise the colonial and neocolonial relationship between scholars from a few developed countries of the North and those from the developing countries of the South, which is now exacerbated by the Digital Divide. The notion of "centres" and "peripheries" in knowledge communication may be an example of power distance that any contextual model for ICT-mediated research communication should seek to address in the effort to come up with a communication system that gives visibility to research outputs from the "peripheries."

Earlier, Mazrui (1978) expounded on the same in his analysis of a peripheral university from an African perspective and proposes three strategies for peripheral universities to counter the domination of knowledge from the centre (the developed North):

a) domestication of imported knowledge to make it relevant to the local culture; 
b) diversification of the knowledge base so that North America and European influences become part of, rather than the only component of, world culture to be adapted to;

c) counter-penetration of periphery scholars into the centre knowledge establishment by nurturing scholarship that is strong enough to give back as much as it takes from Western knowledge, and so leaving a unique contribution to the world knowledge system (Mazrui 1978).

Mazrui's argument that research communication needs to be contextualised to take care of the local culture connects well with Hofstede's (2001) cultural dimensions theory. This is also supported by Newell, et al. (2000), in their article on the diffusion and adoption of complex information technologies in business process re-engineering: "there is a need to unpack this knowledge and integrate it with the localized, context-specific organizational knowledge that is embedded in existing routines and practices” (p. 242). Zhong (1993, p.22), citing Hayhoe (1986; 1989), pushes the power distance debate further by pointing out how inequality in international academic relations is reinforced by legitimisation of both power and knowledge:

...On the one hand, the modern state needs to prove its legitimacy through scientific research, so it is in the state's interest to support the scholarly community. On the other hand, the scholarly community needs to rely on the political support from the state to legitimize its existence, to find funding, and to identify research topics.

Thus, there is a symbiotic relationship between power and knowledge which also exists on the international stage between the collective political power dominated by the First World (the centre) and the international academic/research order (Zhong, 1993), so that the vertical linkage between the centre and periphery is reinforced by the level of technology diffusion and adoption and the kind of knowledge generated and shared through ICT mediation.

Since knowledge communication goes hand in hand with the technology to facilitate it, the "centres" and "peripheries" concept may also apply to diffusion and adoption of new ICT where the technologically well-endowed North is the "centre" while the poorly endowed South, Africa in particular, is the "periphery". Economic wealth is a major factor in the production and diffusion of a new technology and a prerequisite for the diffusion of the Internet, where individual incomes as well as institutional financial capital are seen as influencing its growth (Hargittai, 1999; Kiiski \& Pohjola, 2002; Oyelaran-Oyeyinka \& Lal, 2005). Rodriguez and Wilson (2000) point to the ICT-inequality nexus by arguing that "when 
a new technology is introduced into a social setting where scarce resources and opportunities are distributed asymmetrically, the greater likelihood is that those with more resources will employ them to gain additional ones, including ICTs” (p. 33). This scenario calls for local strategies, as Mazrui has proposed above, to facilitate diffusion and adoption of ICT, if African research institutions are to benefit from and contribute to the global knowledge economy. This study, which seeks to investigate the factors that affect the needs and priorities for ICT-enabled research communication by researchers in Kenya in agriculture, biology/biotechnology, environmental science and health sciences, is a response in that direction.

Diffusion research points to the need to address the cultural dimension in ICT adoption strategies. For example, Kaye and Little (2004) present three factors which hamper efforts to achieve some sort of universal access to ICT:

- the flawed assumption that there exists some sort of linear chain leading to globalization of ICT;

- failure to reconcile the cultural dimensions with the fact that all ICT is developed within a cultural context; and

- the flaw in assuming that ICT development and access is somehow independent of economic access and development.

Kaye and Little (2004) also point to the possibility of developing another type of cultural divide as a result of emphasising the technological aspect of the digital divide. They see the possibility of a divided society "split between the internationally mobile, technologicallysupported communities and those communities disadvantaged economically and technologically but culturally rich. The failure to bridge this gap may leave society as a whole weakened through lack of access to variety” (p. 5).

The idea of flooding developing countries with modern technologies without looking at their cultural background has been compared with giving someone fish without teaching him how to fish (Morales-Gomez \& Melesse, 1998; Sein \& Harindranath, 2004). However, Musa, et al. (2005) criticise the fish analogy as inadequate in capturing the ICT situation for "technologies are not simple artefacts, and their meaningful use and application depend on factors such as local contents and cultures” (p. 112). Newell, et al. (2000) reiterate the same 
when they say that "innovation processes need increasingly to be seen as interactive, as they involve the integration of knowledge across disparate social communities” (p. 255).

Thus, there is need to understand the socio-cultural factor in the adoption of ICT for scientific research communication and Hofstede provides the lenses through which one can probe for such detail.

\subsection{The Research Framework}

This study investigated the enablers for and barriers, as a means to developing a contextual framework of factors for ICT-enabled research communication by researchers in Kenya in agriculture, biology/biotechnology, environmental science and health sciences.

To make sense of this intricate phenomenon required a sound theoretical framework to provide a basis for describing the pattern of the adoption. Consequently, the tentative research model for this study was informed by perspectives from the literature, DOI and Hofstede's Cultural Dimensions frameworks, and located in a Kenyan context. The perspectives were used as theoretical lenses to guide the research study in order to facilitate collection of rich data that could provide for in-depth explanations and thick descriptions pertaining to the enablers and barriers to adoption of ICT in communication of research output by researchers in Kenya in the selected fields. This would form a basis for the contextual framework for ICT-enabled research communication by researchers in Kenya.

The researcher used the variables in Rogers' DOI theory (see 3.1.1.1 above) and Hofstede's Cultural Dimensions theory, discussed under section 3.1.2 above, to investigate the organisational, socio-economic, cultural, and technological factors that affect the adoption of ICT for the communication of Kenyan research output as a means to greater accessibility, visibility and participation in the global knowledge economy. The literature review in chapter two already pointed to the need for an understanding of these dimensions as a precursor to development of a contextual model for an ICT-enabled research communication. This indicated a gap in literature that led to the research objectives and questions as reflected in figure 3.2 and outlined in table 3.3 below. 
The justification for choosing the two models has already been given in the previous sections (see 3.1.1.2, 3.1.2.2. and 3.1.2.3). The two models have been chosen because they complement each other and have been used in many contexts in both quantitative and qualitative studies as already discussed earlier (see sections 3.1.1.2 - 3.1.2.2). Whereas Rogers (2003) guides the social, organisational and technological, he does not address the issues of culture which Hofstede $(1980,1991,2001)$ does. Therefore, in order to come up with a robust framework, the researcher chose to use DOI together with the Cultural Dimensions model to investigate the cultural interplay affecting the diffusion, adoption and use of ICT, which the researcher considered vital in developing a contextual framework that is specific to the Kenyan context (See figure 3.2 below). 


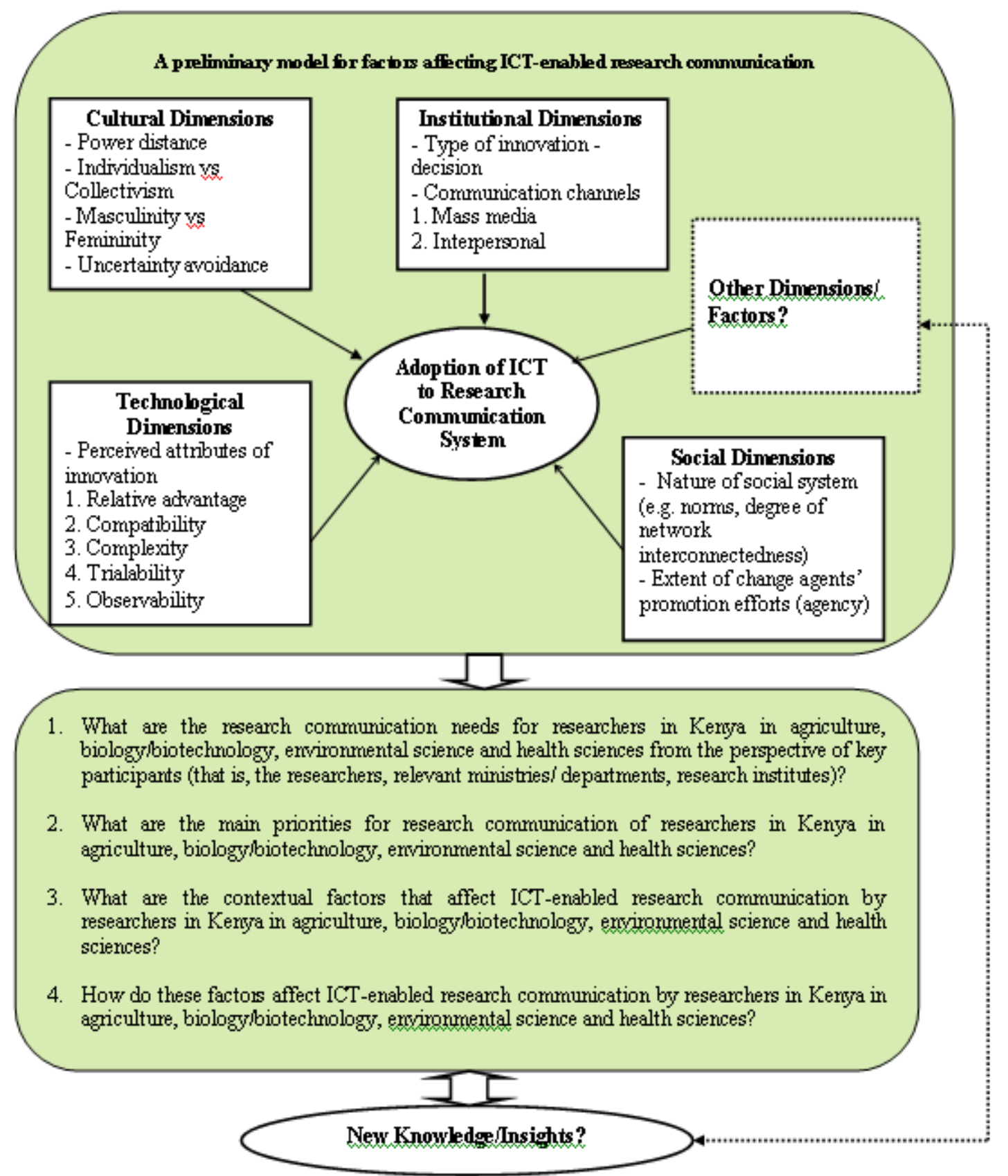

Figure 3.2: A proposed framework for investigating factors affecting diffusion and adoption of ICT for research communication in Kenya

Figure 3.2 Proposed framework for investigating factors affecting diffusion and adoption of ICT for research communication in Kenya

The preliminary model is in line with the first objective of the study - "To develop a preliminary model of factors that affect ICT-enabled research communication" - as per the literature and relevant theories on diffusion of new innovations/technologies. The second and third objectives - "To identify the communication needs and identify priorities of 
communication needs for researchers in Kenya in agriculture, biology/biotechnology, environmental and health sciences” and “To identify and understand enablers for and barriers to ICT-enabled research communication by researchers in Kenya in agriculture, biology/biotechnology, environmental and health sciences" - led to the development of the research questions reflected in figures 3.2 above. The research questions provided for the collection of data that could lead to a contextual model for ICT-enabled research communication by researchers in Kenya that is informed by the researchers' research communication needs and priorities (see ellipse at the bottom of framework - New Knowledge/Insights in figure 3.2 that would lead to a revision of the preliminary model as indicated by the dotted line.

The link between the research objectives and research questions is illustrated in table 3.3 below.

Table 3.3: Link between research objectives and research questions

\begin{tabular}{|c|c|}
\hline Research objective & Research question \\
\hline $\begin{array}{l}\text { To develop a preliminary model of factors that affect } \\
\text { ICT-enabled research communication }\end{array}$ & - \\
\hline \multirow[t]{2}{*}{$\begin{array}{l}\text { To identify the communication needs and establish } \\
\text { priorities of communication needs for researchers in } \\
\text { Kenya in agriculture, biology/biotechnology, } \\
\text { environmental science and health sciences. }\end{array}$} & $\begin{array}{l}\text {-What are the research communication needs for } \\
\text { researchers in Kenya in agriculture, } \\
\text { biology/biotechnology, environmental science and } \\
\text { health sciences from the perspective of key participants } \\
\text { (that is, the researchers, ministries/departments, research } \\
\text { institutes)? }\end{array}$ \\
\hline & $\begin{array}{l}\text {-What are the main priorities for research } \\
\text { communication of researchers in Kenya in agriculture, } \\
\text { biology/biotechnology, environmental science and } \\
\text { health sciences? }\end{array}$ \\
\hline $\begin{array}{l}\text { To identify and understand enablers for and barriers } \\
\text { to ICT-enabled research communication by } \\
\text { researchers in Kenya in agriculture, } \\
\text { biology/biotechnology, environmental science and } \\
\text { health sciences. }\end{array}$ & $\begin{array}{l}\text {-What are the contextual factors that affect ICT-enabled } \\
\text { research communication by researchers in Kenya in } \\
\text { agriculture, biology/biotechnology, environmental } \\
\text { science and health sciences (for example, with regard to } \\
\text { the relevant organisations, the research environment, } \\
\text { research infrastructure, etc.)? } \\
\text {-How do these factors affect ICT-enabled research } \\
\text { communication by researchers in Kenya in agriculture, } \\
\text { biology/biotechnology, environmental science and } \\
\text { health sciences? }\end{array}$ \\
\hline
\end{tabular}


To make it possible to collect data that could provide answers to meet the research objectives, the researcher developed an interview schedule in line with the gaps revealed by a review of the literature and relevant theories, as reflected in the dimensions of the preliminary model framework and the research questions in figure 3.2 above. The interview questions sought to elicit information that could provide an understanding of the socio-cultural, organisational, technological and other factors that could be enablers and/or barriers to adoption of ICT for research communication. This was achieved by asking what and how questions (see table 3.4 below), whose responses led the researcher to probe for further detailed explanations (why of ICT-enabled research communication). This resulted in rich data that led to an in-depth description of the factors that affected the adoption of ICT for research communication by researchers in Kenya. 


\title{
Table 3.4: Interview Schedule
}

\author{
A. Organisational factors \\ 1. a) What are the challenges facing communication of research output in your institution? \\ b) What has caused these challenges?
}

2. What are the communication needs of researchers in your organisation?

3. Among these communication needs, what are the priorities?

4. a) What research dissemination strategies exist in your research institution?

b) To what extent do these strategies affect the adoption of ICT in the research communication process?

5. a) What research dissemination policies are in place in your research institution?

b) To what extent do these policies affect the adoption of ICT in the research communication process?

6. a) What communication channels do researchers in your research institution have access to?

b) How are the communication channels changing through the mediation of ICT? c) What contributions is ICT-enabled research communication likely to make towards the realisation of the universal axiom or virtue of the scholarly motto of "publish or perish" within the research community in your institution?

\section{B. Technological factors}

1. What ICT do you have access to?2. How would you describe their accessibility in terms of:

a) Ease of use?

b) Compatibility with your work?

c) Trialability?

d) Relative advantage over other technologies?

e) Reliability?

3. What do you, as a researcher/journal editor/librarian, perceive as the benefits and disadvantages of ICTmediated research communication/dissemination?

\section{Social factors}

1. a) What social norms in relation to research communication obtain in the institution?

b) How do they affect adoption and use of ICT for research communication?

2. a) What social networks exist?

b) What role do they play in facilitating ICT adoption and use?

D. Cultural Dimensions

1. How do national value systems affect the adoption and use of ICT for communication of research output?

2. What role do other national-level factors play in the adoption and use of ICT for research communication?

\section{E. Other Dimensions}

1. What does your research involve?

2. What type of resources do you require to disseminate your research output?

3. How does the nature of your research and types of resources required affect the adoption and use of ICT for communication of research?

Details on how data were gathered to answer the research questions are discussed in the next chapter. 


\section{CHAPTER FOUR}

\section{STUDY DESIGN AND METHODOLOGY}

\subsection{Chapter Overview}

This chapter addresses issues to do with the research paradigm adopted for this research and the methods used to undertake data collection in order to answer the research questions raised in chapter one. It also reports on the measures taken to safeguard research validity and trustworthiness.

\subsection{Interpretivist Paradigm}

The literature points to many philosophical paradigms (the researcher's interpretive frameworks or worldviews) that underpin qualitative research. The research paradigm adopted guides the way the researcher conceives the research objectives, approaches the research process, and ultimately contributes to the research outcome. Orlikowski and Baroudi (1991), following Chua (1986), as cited in Myers (1997), suggest three categories: positivist, interpretive and critical. Guba and Lincoln (1994) suggest four paradigms: positivism, postpositivism, critical theory, and constructivism.

Positivism assumes that reality is objectively given and can be represented by measurable properties, which are independent of the researcher and his/her instruments. It attempts to test theory in order to increase the predictive understanding of phenomena (Myers, 1997). IS research can be said to be positivist if there is evidence of formal propositions, quantifiable measures of variables, hypothesis testing, and the drawing of inferences about a phenomenon from the sample to a stated population (Orlikowski \& Baroudi, 1991). Critical research, on

the other hand, sees social reality as historically constituted and produced and reproduced by people. It seeks to provide a social critique, exposing inequities and conflicts in society with the aim of emancipating or helping humans to realise their potential through eliminating the causes of inequality and domination.

The researcher in this study adopted an interpretivist stance, which is based on the view that there is no universal truth and, therefore, regards reality as an essentially subjective social product that is constructed and interpreted by humans according to their beliefs and value 
systems (Bryman, 2004; Creswell, 2003; Denzin \& Lincoln, 2005; Myers, 1997). Interpretive research attempts to understand phenomena through the meanings that the research participants assign to them. It focuses on the full complexity of human sense-making as a situation emerges (Kaplan \& Maxwell, 1994, cited in Myers (1997).

An interpretivist stance was chosen for this research study because it has become an important research tradition in IS, which has the potential to produce deep insights into IS phenomena, as noted by Klein and Myers (1999). The researcher sought to explore and understand factors affecting the diffusion and adoption of ICT for research communication in the context of the Kenyan research institutions in the area of applied science and technology. Consequently, the researcher considered the interpretivist paradigm the most suitable for this study because of its subjective stance and view of the world as socially constructed through human perceptions and interactions. This enabled the researcher to understand what the various stakeholders in the research study considered to be important communication needs and what the researchers viewed as priorities in research communication. It also enabled the researcher to understand what the stakeholders believed: how they felt and interpreted events pertaining to adoption and use of ICT for research communication. In addition, this stance suited the types of social interactions that occur in the processes of diffusion and adoption of new innovations like ICT and knowledge communication and dissemination. These processes do not exist independently, but are heavily people-centred and, therefore, needed a philosophical stance that could help capture, in depth, the different views surrounding the adoption of ICT for the research communication process.

\subsection{Qualitative Research Design}

This study sought to contribute to the understanding of ICT-enabled research communication and dissemination by and for scholars and researchers working in Kenya. The main purpose was to identify barriers to and enablers for ICT-enabled research dissemination. Because of the embeddedness to the organisational and non-organisational environments of the phenomenon being investigated, the study used qualitative research design and methods to help understand the factors affecting adoption and use of ICT for research communication by and for researchers in Kenya within their social and cultural contexts. 
The qualitative design was chosen for this study because its inductive approach emphasises the qualities of entities and processes and meanings that are not experimentally examined or measured in terms of quantity, amount, intensity, or frequency. Qualitative researchers stress the socially constructed nature of reality, the intimate relationship between the researcher and what is studied, and the situational constraints that affect the inquiry (Bryman, 2004; Denzin \& Lincoln, 2005). This is unlike quantitative research that entails a deductive approach to the relationship between theory and research in which the accent is placed on the testing of theories, incorporates a positivist epistemological position and embodies a view of social reality as an external, objective reality (objectivist ontology) within a value-free framework. Hence, this design fitted well with the goal of the study, which was to gain an understanding of the socio-cultural, organisational, technological and other factors affecting the adoption of ICT for research communication with a view to developing a contextual model for ICTenabled research communication based on the experience, perceptions, and priority communication needs of researchers in applied sciences in research institutions in Kenya (Bryman, 2004; Powell \& Connaway, 2004).

The researcher sought to gain insights into the intricate processes of ICT adoption to the research communication process through close interactions with the research participants. This was a subjective process, only possible through a qualitative research design where the researcher adopts an interpretivist epistemological position that stresses the need for an understanding of the social world through an examination of the interpretation of that world by its participants. This involves adopting a constructionist ontological position that views social properties as outcomes of the interactions between individuals, rather than phenomena 'out there' separate from those involved in their construction (Bryman, 2004). Therefore, unlike in quantitative research theory formation becomes an organic, continuous process designed to understand phenomena (Bryman, 2004; Powell \& Connaway, 2004) such as ICTenabled research communication. Thus, the recursive nature of qualitative research allows for capturing the changing and emerging reality about phenomena and processes (see figure 4.1 below). As such, the approach provided offered insights into information-seeking experiences, as the researcher sought information from research participants regarding the barriers to and enablers for ICT adoption to the research communication process. 
Another basis for adopting qualitative design and methods in this study was Myers' (1997) argument that there has been a general shift in IS research away from the technological to managerial and organisational issues, which has increased the pull towards the application of qualitative research methods. This shift is reiterated by Flick (2002, cited in Denzin \& Lincoln, 2005), who says the rapid social change and the resulting diversification of life worlds that increasingly confront social contexts and perspectives cannot be adequately captured by deductive methodologies, thus, forcing research to adopt inductive strategies instead of starting from theories and testing them. He explains that "knowledge and practice are studied as local knowledge and practice” (Flick, 2002, cited in Denzin \& Lincon, 2005, p.11). This study was about establishing barriers to and enablers for adopting ICT to facilitate communication of local scientific research output in Kenya in order to contribute to local knowledge and practice in the Kenyan/African context. It is, therefore, clear from the above argument that the qualitative design adopted in this study was the best suited to achieve that.

The qualitative approach was also appropriate for this research because, while there may be studies on the capabilities of ICT, there is little contextual literature pertaining to the enablers for and barriers to ICT-enabled research communication. The flexibility of the qualitative design, therefore, enabled the researcher to draw data from the context in which the research communication and ICT adoption occurred, in order to be able to describe these occurrences as a means of determining the process in which ICT-enabled research communication is embedded, using induction to derive possible explanations from participants' views and personal observation of how they went about the research communication phenomena. This was in line with views of many qualitative research proponents such as Bryman (2004); Creswell (2003); Gary \& Clayton (2005); and Denzin \& Lincoln (2005). 


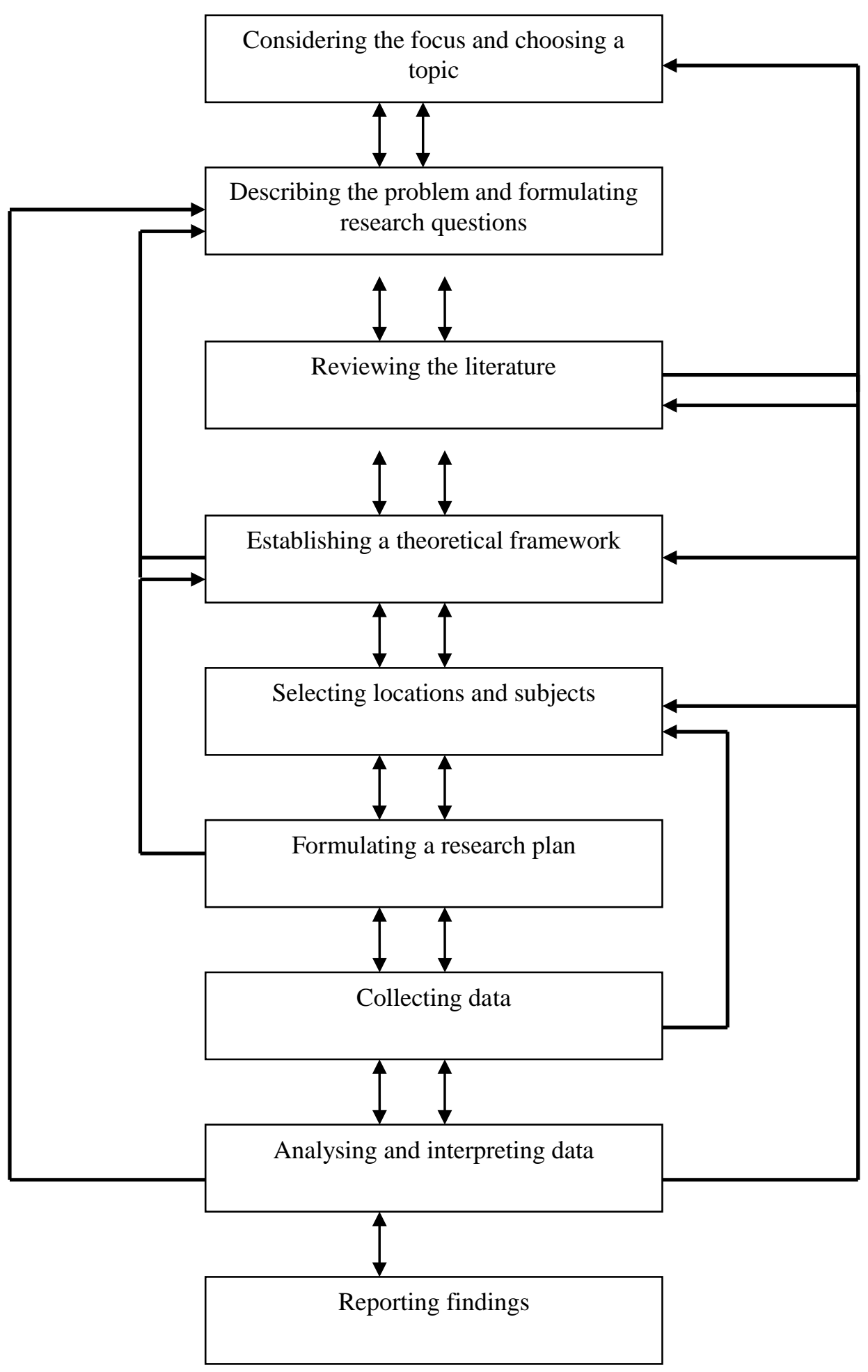

Figure 4.1: The recursive research process (Gorman and Clayton 2005, p. 37): 


\subsection{The Role of the Researcher}

Interpretive research has long been an object of criticism owing to the prominence of the researcher in the research process and interpretation of results, which presents the possibility of researcher bias. Unlike positivist enquiry, whose goal is objectivity owing to its view of reality as single, tangible, fragmentable and value-free, interpretive research is subjective and acknowledges that there are multiple realities and that the researcher and the study participants mutually influence and shape a reality (Gorman \& Clayton, 2005). Objectivity in interpretive research is viewed as impossible and researcher bias is a resource rather than a weakness of a study in that, when joined with the perceptions of study participants, the researcher's bias contributes to a new, mutually constructed reality (Gorman \& Clayton, 2005). Wolcott (1995) views bias as crucial to an investigation because "researcher bias is a manifestation of the cultural self" (cited in Gorman \& Clayton (2005, p.191).

To control and deal with the potential for bias in an investigation, the interpretive researcher is expected to apply the practice of building trustworthiness. As an interpretive researcher, one must be candid about personal perspectives and experiences in the "person-as-researchinstrument” statement (Creswell, 2003; Gorman \& Clayton, 2005)(Creswell 2003; Gorman and Clayton 2005). In this study, the researcher adopted an outside observer role. This was possible because the researcher was a mainstream civil servant, in a Ministry that has no link with the research institutions and their parent ministries. Apart from limited interaction with librarians and journal editors in some of the research institutions as a research fellow for UNESCO/Keizo Obuchi in 2002/03, the researcher had no personal stake in the interpretations and outcomes during the research process. Hence, this could be viewed as an advantage in that it placed her in a unique position to address the problem pertaining to ICT adoption for research communication and also put the participants at ease to frankly give their opinions, especially after establishing good rapport with the respondents. However, this external role could prevent the researcher from getting a sense of the research setting from the inside, or obtaining access to confidential or sensitive data and issues (Walsham, 1995). Also, the researcher's role as a programme officer in a Ministry that has been associated with rationalisation and staff retrenchment made some participants unwilling to divulge information. Nevertheless, the advantages outweighed the disadvantages. 
There was also the issue of the researcher's cultural self and national background. Being a Kenyan who has grown and shared in the culture of the research participants made it easy for the researcher to understand and explain issues related to the socio-cultural environment, such as the social/communal and oral nature of Kenyans pitted against the isolationist and silent nature of computer-mediated communication; the high power distance in relationships between those highly titled/qualified in academic terms but low in ICT skills and the ICT savvy but lower qualified/students, as well as issues to do with age and gender. On the other hand, culture familiarity might lead the researcher to interpret issues based on her own experience and not that of the participants. To avoid such a scenario, the researcher was always careful to document the views of the research participants and use them to support all claims and conclusions.

Given that the researcher was not a research scientist, the science-oriented research institutions setting and the process of communication of scientific research outputs posed challenges and advantages. The challenges arose in that the researcher could not easily understand the concepts and issues that related to scientific research communication and its day-to-day constraints in the particular institutions. Though the researcher did not encounter such a scenario, there was the possibility of limited trust on the part of participants regarding her ability to articulate issues pertaining to scientific communication, as well as concerns about the usefulness of the final research report. To guard against such issues, the researcher was ever alert for nuances, and constantly reflected on and requested validation of emerging understanding and issues. Conversely, her outsider's perspective was viewed as providing benefits in that factors unique to the scientific cultural setting relating to the adoption of ICT for research communication were easier to perceive. Moreover, the researcher was seen as relatively free from assumptions associated with the scientific research fraternity, thus, in a unique position to uncover issues that the insiders could not.

The literature review identified conceptual gaps and theoretical models that could be applied in gathering data to fill the gaps. However, the researcher used the models only as lenses and did not restrict herself to their prescriptions at the expense of new insights that arose in the course of the study. She was careful to balance following possible leads from the literature with attempts to unearth emergent findings impacting on the adoption of ICT for the 
communication of scientific research outputs. To ensure checks and balances in the practice of building credibility and trustworthiness, the research applied Lincoln and Guba's techniques:

- Undertook prolonged engagement in the field to ensure collection of in-depth data/information.

- Was persistent in her observations to be able to identify relevant events and their relationships as every setting had unique features and dynamics.

- Applied more than one method of data sources, settings and research participants to help ensure legitimacy of the observations and to help in seeing phenomena from a variety of perspectives.

- Was modest enough to seek peer debriefing to help review perceptions, methods and analytical techniques and also get additional and expert perspective as events unfolded.

- Allowed for member checking or review to confirm data and their interpretation and also to help in overcoming certain aspects of research bias (Gorman \& Clayton, 2005; Lincoln \& Guba, 1985).

\subsection{Data Gathering Techniques}

This study used qualitative data collection methods to collect data to answer the research questions. These were chosen because of their focus on naturally occurring, ordinary events in their natural settings (Miles \& Huberman, 1994, p.10) and were, therefore, viewed as being most suitable for eliciting an in-depth understanding of a phenomenon, especially where little was known about what was being investigated, as was the case in this study where there was little contextual literature pertaining to diffusion and adoption of ICT for research communication in Kenya. In addition, qualitative methods are inherently flexible and could be varied in the course of the study to allow for newly emerging and divergent themes (Powell \& Connaway, 2004). Thus, data gathering was envisioned as a process rather than a procedure, in that it required analytic judgement rather than a preplanned routine (Bryman, 2004; Creswell, 2003; Denzin \& Lincoln, 2005; Gorman \& Clayton, 1997, 2005; Powell \& Connaway, 2004). 
Also, the predilection of qualitative methods to see through the eyes of the people being studied helped the researcher to probe beneath surface appearances pertaining to diffusion and adoption of ICT for research communication, resulting in rich, holistic and complex information and meanings (Bryman, 2004). In IS studies, interpretive methods of research are "aimed at producing an understanding of the context of the information system, and the process whereby the information system influences and is influenced by the context" Walsham (1993, pp.4-5). Miles and Huberman (1994) see qualitative methods as the best strategy for exploring a new area and generating and revising conceptual frameworks. Hence, qualitative methods were ideal for this research study, which sought to develop a contextual framework for ICT-enabled scientific research communication in Kenya.

\subsubsection{The research samples}

The purpose of this research was to understand the factors affecting, and how they affected, the adoption and use of ICT in scientific research communication among scholars and researchers in Kenya. Consequently, the researcher employed the purposive sampling technique in selecting the research sites and research participants. Bradley (1993) argues, "In purposeful sampling, members of the sample are deliberately chosen based on criteria that have relevance to the research question rather than criteria of randomness of selection” ( $p$. 440).

\subsubsection{Research Sites}

The researcher purposively sampled research sites that were relevant to the goal of the research study before going to the field. These included research institutions in Kenya that are undertaking research in some of the government's key priority areas in the economic recovery as outlined in the ERSWEC 2003-2007 and current Kenya Vision 2030 (Government of Kenya, 2007d). The areas encompassed applied sciences and technology, specifically the fields of agriculture, biology/biotechnology, environmental science and health sciences. Altogether eight (8) research institutions whose composition included three (3) research national institutes, two (2) national universities and three (3) international research institutes were chosen. Owing to confidentiality required by the Victoria Human Ethics Committees when dealing with human subjects, the institutions are referred to in this thesis by letters of 
the alphabet. For example, the two national universities are allotted the synonyms A and B while the three national research institutes are $\mathrm{C}, \mathrm{D}$ and $\mathrm{F}$. The international research institutes are referred to as E, G and $\mathrm{H}$. Only those research institutes and universities dealing with agriculture, biology/biotechnology, environmental science and health sciences were selected. Although there are other universities undertaking research in the specified disciplines, A and B were chosen because of their long-standing history in applied science and technology research in Kenya.

Sampled and selected also were five (5) ministries/departments under which the research institutions were affiliated. For sake of anonymity, these ministries/ departments are referred to as $\mathrm{M}$ and numbered using the alphabet.

\subsubsection{Sample population}

In deciding on the sample size, the researcher was guided by the quality of the data sources in terms of depth, complexity, and "richness”, as Powell and Connaway (2004) put it: “No single formula provides the "correct" sample size for a qualitative study. (p.189) (See also (Bradley, 1993; Bryman, 2004; Creswell, 2003; Denzin \& Lincoln, 2005; Gorman \& Clayton, 1997, 2005). Also guiding the researcher were the three factors common in many qualitative studies:

- the standard of saturation where one continues gathering data until critical elements of the study have become "saturated" or until further exemplars fail to add new nuances or to contradict what is understood;

- $\quad$ the nature of the research question being asked; and

- the purpose of the first sample, used to gather initial data based on which "the first sample may be adjusted, augmented, enlarged, or otherwise modified to meet the new parameters” (Powell \& Connaway, 2004, p. 190). In this study, the researcher used in the first sample to gauge the kind and depth of information/data the interview guidelines were likely to elicit from the research participants, following which she accordingly revised the research guidelines. 
The sample population of research participants depended on the size of the research institutions and areas of focus. However, by applying stratified purposive sampling, the researcher included representatives from within the population being studied (researchers, senior administrators, science editors and librarians) who had a range of characteristics relevant to the research project (Gorman \& Clayton, 2005). As mentioned earlier in chapter 1 (see 1.4), these groups of participants are central in the research communication process in Kenya:

- Researchers and scholars undertake the research that produces the content that editors edit in preparation for dissemination through journals. In selecting the researchers to participate in the study, care was taken to include the perspectives of both older, experienced researchers and younger, less experienced ones. Twenty-four (24) researchers, three (3) of whom doubled as science editors, were interviewed.

- Science editors edit scholars and researchers' work before it is communicated using available channels. The researcher focused on science editors cum communicators in the specific institutions. Five (5) science editors, of who three (3) doubled as researchers, were interviewed.

- Senior administrators formulate and implement policies and make important decisions on budget allocations that affect the research process and introduction of any new innovation. Hence, they were sampled to explore their views on communication of research and issues regarding policy on funding allocation for ICT-enabled research communication. This sample comprised the following:

- Permanent Secretaries and directors from ministries/departments relevant to the focus of the study, which were also the parent ministries of the research institutions under investigation (6), and one unplanned focus group of eight (8) officers from Ministry1;

- Directors/ Heads of departments of research institutes and relevant faculty deans in the two universities (8).

Altogether, 21 administrators were interviewed (including the focus group of 8). 
- Research librarians support researchers in their efforts to create content and at the same time serve to disseminate the research results to those who seek to use them. Under this category, the researcher sampled head librarians in the two selected universities, two government and two international research institutes (six (6) in number). This position participates in policy and planning issues and, therefore, the group is likely to bear the responsibility for negotiating ICT-related issues, as regards the acquisition, communication and dissemination of research information materials.

For confidentiality reasons, government officers are referred to as GO and numbered using numerals; both the directors and researchers and scholars are referred to as RS (research scientist) followed by a numeral if working in a research institute or LR (lecturer researcher) followed by a numeral if working in a university. The science editors are referred to as SE and the librarians as L, each respectively followed by a numeral (see table 4.1 below for a summary of the sample population by category). 
Table 4.1: Sample population

\begin{tabular}{|c|c|c|c|c|c|}
\hline Institution & Heads of Dept. & $\begin{array}{c}\text { Scholars (LR)/ } \\
\text { Researchers (RS) }\end{array}$ & $\begin{array}{l}\text { Librarians } \\
\text { (L) }\end{array}$ & $\begin{array}{c}\text { Science } \\
\text { editors } \\
\text { (SE) }\end{array}$ & $\begin{array}{l}\text { TOTAL no. of } \\
\text { interviews per } \\
\text { institution }\end{array}$ \\
\hline $\mathrm{D}$ & 1 & 4 & 1 & - & 6 \\
\hline $\mathrm{C}$ & 1 & 4 & 1 & - & 6 \\
\hline $\mathrm{H}$ & 1 & 2 & 1 & - & 4 \\
\hline E & 1 & 2 & 1 & - & 4 \\
\hline G & 1 & 2 & - & - & 3 \\
\hline $\mathrm{F}$ & 1 & 2 & - & - & 3 \\
\hline B & 1 & 4 & 1 & - & 6 \\
\hline A & 1 & 4 & 1 & - & 6 \\
\hline Science editors & - & - & - & 6 (3 RS) & 6 \\
\hline $\begin{array}{l}\text { Ministries/ } \\
\text { departments 1-6 } \\
\text { (GO) }\end{array}$ & $\begin{array}{l}13 \text { (including } \\
\text { one unplanned } \\
\text { focus group of } \\
8 \text { ) }\end{array}$ & - & - & & 6 \\
\hline $\begin{array}{l}\text { Total per } \\
\text { category of } \\
\text { participants }\end{array}$ & 21 & 24 (3 SE) & 6 & 6(3 RS) & - \\
\hline \multicolumn{5}{|c|}{ GRAND TOTAL } & 54 \\
\hline
\end{tabular}


Sampling and interviewing people in different organisational positions and related organisations helped in gaining a variety of perspectives regarding barriers to and enablers of ICT-enabled research communication. It also served to triangulate the information gathered.

The researcher used two general approaches to purposive sampling:

1) Snowball sampling was used to lead the researcher to individual researchers and science editors with shared experiences and perspectives in the same population about barriers and enablers to ICT-enabled research communication;

2) Theoretical sampling - entails sampling interviewees until your categories achieve theoretical saturation, then selecting further interviewees on the basis of your emerging theoretical focus. The approach is iterative - one in which there is a movement backwards and forwards between sampling and theoretical reflection. The chief virtue of theoretical sampling is that it emphasises using theoretical reflection on data as a guide to whether more data are needed. It, therefore, places a premium on theorising rather than the statistical adequacy of a sample, which may be a limited guide to sample selection in many instances (Bryman, 2004). Here, the issues addressed by the research questions and elements of the preliminary research framework guided the researcher in judging the adequacy of the data gathered. To achieve this, the researcher continuously analysed the data to establish how well they were answering the research questions and the theoretical models guiding the study and continued sampling until there was a feeling saturation had been reached.

\subsubsection{Document analysis}

The researcher used document analysis technique to gather data relating to ICT status in Kenya, existing policies/ strategies and regulatory and legal framework, ICT infrastructure, and challenges on ICT adoption and use for research communication by and for researchers in the sampled research institutions. She gathered and reviewed selected documents from selected institutions and government ministries/departments in the sampled institutions. The range of documents collected included: 
1) Institutional documentation- annual reports, performance appraisal reports, strategic plans, institutional policies and strategies on ICT and research communication, research reports, reviews, evaluations, published and unpublished materials;

2) External documentation - government policies and strategies on ICT and research communication, research reports, published and unpublished materials.

The gathering of the documents was undertaken along with the interviews. The researcher requested relevant documentation from interviewees during the interviews. She also visited the libraries and websites of the research institutions and government ministries/departments for information on ICT and research communication in the specific institutions. This sought to complement and triangulate data gathered through the interviews; thus, adding validity to the study.

\subsubsection{Interview technique}

Guided by semi-structured, open-ended questions, the researcher held in-depth face-to-face interviews. This was intended to keep structure to a minimum, in order to enhance the opportunity for genuinely revealing the perspectives of the people being studied. The researcher interviewed respondents who had a range of characteristics (researchers, administrators, government representatives, librarians and journal editors) relevant to the research project. This assisted in checking the reliability/authenticity of the information/data gathered which added to the validity. The semi-structured interview guidelines helped bring out aspects of participants' social world, which were particularly important to them.

In-depth face-to-face interviewing techniques were chosen to allow for additional explanation, counter questions and even open discussion. The interviews were conducted in English, which is the official language in Kenya. To capture the information, the researcher tape-recorded and transcribed the interviews. Recording allowed for complete account of series of exchanges in the interviews to be available and to be fully woven into the analysis. It also enabled the researcher to concentrate on the interviewing and not be distracted by having to write notes on what was being said.

Heritage (1984) gives the advantages of recording and transcribing as: 
- helping to correct the natural limitations of our memories and of the intuitive glosses that we might place on what people say in interviews;

- allowing more thorough examination of what people say;

- permitting repeated examination of the interviewees' answers;

- opening up the data to public scrutiny by other researchers, who can evaluate the analysis that is carried out by the original researchers of the data (that is, secondary analysis);

- helping to counter accusations that an analysis might have been influenced by a researcher's values or biases;

- allowing the data to be reused in other ways from those intended by the original researcher - for example, in the light of new theoretical ideas or analytic strategies (cited in Bryman (2004, p.330).

Table 4.2 shows the links between research questions, data collection techniques and data types. 
Table 4.2: Links between research questions, data collection techniques and data types

\begin{tabular}{|c|c|c|}
\hline Research Question & Research Method & What Data? \\
\hline $\begin{array}{l}\text { 1) What are the research communication } \\
\text { needs for researchers in Kenya in } \\
\text { agriculture, biology/biotechnology, } \\
\text { environmental science and health sciences } \\
\text { from the perspective of key participants } \\
\text { (that is, the researchers, government } \\
\text { ministries/departments, research institutes)? }\end{array}$ & $\begin{array}{l}\text { - In-depth face-to-face interviews with } \\
\text { researchers in the selected disciplines and } \\
\text { institutions } \\
\text { - Document analysis of existing documentary } \\
\text { material: } \\
\text { 1) Institutional documentation - annual reports, } \\
\text { performance appraisal reports, strategic plans, } \\
\text { policies \& strategies, research reports, reviews, } \\
\text { evaluations } \\
\text { 2) External documentation - government } \\
\text { policies, strategies, research reports, published } \\
\text { and unpublished material. }\end{array}$ & $\begin{array}{l}\text { - Insights from the researchers on what they } \\
\text { see as their most important communication } \\
\text { needs } \\
\text {-Documentary evidence from annual and } \\
\text { technical reports; government policies \& } \\
\text { strategies; strategic plans, etc. }\end{array}$ \\
\hline $\begin{array}{l}\text { 2) What are the main priorities for research } \\
\text { communication of Kenyan researchers in } \\
\text { agriculture, biology/biotechnology, } \\
\text { environmental science and health sciences? }\end{array}$ & $\begin{array}{l}\text {-In-depth face-to-face interviews with } \\
\text { researchers in the selected disciplines and } \\
\text { institutions } \\
\text {-Document analysis of existing documentary } \\
\text { material: } \\
\text { 1) Institutional documentation- annual reports, } \\
\text { performance appraisal reports, strategic plans, } \\
\text { policies \& strategies, research reports, reviews, } \\
\text { evaluations } \\
\text { 2) External documentation - government } \\
\text { policies, strategies, research reports, published }\end{array}$ & $\begin{array}{l}\text { - Insights from researchers on what they } \\
\text { feel are their priorities for research } \\
\text { communication } \\
\text { - Documentary evidence from annual and } \\
\text { technical reports; government policies \& } \\
\text { strategies; institutions' strategic plans, etc. }\end{array}$ \\
\hline
\end{tabular}




\begin{tabular}{|c|c|c|}
\hline Research Question & Research Method & What Data? \\
\hline & and unpublished material. & \\
\hline $\begin{array}{l}\text { (3) What are the contextual factors that } \\
\text { affect ICT-enabled research } \\
\text { communication by researchers in Kenya in } \\
\text { agriculture, biology/biotechnology, } \\
\text { environmental science and health sciences } \\
\text { (for example, with regard to the relevant } \\
\text { organisations, the research environment, } \\
\text { research infrastructure, etc.)? }\end{array}$ & $\begin{array}{l}\text {-In-depth face-to-face interviews with } \\
\text { researchers; research institutions' } \\
\text { administrators; representatives from relevant } \\
\text { government Ministries/departments; librarians } \\
\text { and science editors } \\
\text {-Document analysis of existing documentary } \\
\text { material: } \\
\text { 1) Institutional documentation- annual reports, } \\
\text { performance appraisal reports, strategic plans, } \\
\text { policies \& strategies, research reports, reviews, } \\
\text { evaluations } \\
\text { 2) External documentation - government } \\
\text { policies, strategies, research reports, journal \& } \\
\text { newspaper articles, etc. }\end{array}$ & $\begin{array}{l}\text {-Insights on institutional, social, cultural } \\
\text { and technological factors affecting } \\
\text { diffusion, adoption and use of ICT in } \\
\text { research communication from the various } \\
\text { stakeholders } \\
\text {-Documentary evidence from annual and } \\
\text { technical reports; government policies \& } \\
\text { strategies; strategic plans; newspapers; } \\
\text { journals, etc. }\end{array}$ \\
\hline $\begin{array}{l}\text { 4) How do these factors affect ICT- } \\
\text { enabled research communication by } \\
\text { researchers in Kenya in agriculture, } \\
\text { biology/biotechnology, environmental } \\
\text { science and health sciences? }\end{array}$ & $\begin{array}{l}\text {-In-depth face-to-face interviews with } \\
\text { researchers; research institutions' } \\
\text { administrators; representatives from relevant } \\
\text { government Ministries/departments and } \\
\text { research councils; librarians and science dditors } \\
\text { - Document analysis of existing documentary } \\
\text { material: } \\
\text { 1) Institutional documentation- annual reports, } \\
\text { performance appraisal reports, strategic plans, } \\
\text { policies \& strategies, research reports, reviews, }\end{array}$ & $\begin{array}{l}\text { - Insights on enablers for and barriers to } \\
\text { diffusion, adoption and use of ICT for } \\
\text { research communication from the various } \\
\text { stakeholders } \\
\text { - Documentary evidence from annual and } \\
\text { technical reports; government policies \& } \\
\text { strategies; strategic plans; newspapers; }\end{array}$ \\
\hline
\end{tabular}




\begin{tabular}{|l|l|l|}
\hline \multicolumn{1}{|c|}{ Research Question } & \multicolumn{1}{|c|}{ Research Method } & \multicolumn{1}{|c|}{ What Data? } \\
\hline & evaluations & journals, etc. \\
& 2) External documentation - government \\
& $\begin{array}{l}\text { policies, strategies, research reports and journal } \\
\text { articles. }\end{array}$ & \\
\hline
\end{tabular}

Table 4.2: Links between rese arch questions, data collection techniques and data types 


\subsubsection{Interview procedure}

Care was taken to observe ethical issues as required by the Victoria University School of Information Management Human Ethics Committee (HEC). The researcher had applied for and been granted HEC approval, which included preparing the following documents:

- Information sheet appraising participants with what the research was about and their rights pertaining to the participation in the study;

- Consent form for the willing participants to sign; and

- A letter of introduction to heads of the institution explaining the purpose of the study and seeking their permission to allow undertaking of the research among their staff in their institutions.

On arrival in Kenya, the researcher also applied for a research permit from the then Ministry of Education, Science and Technology to seek clearance to undertake the research in Kenyan institutions, as required by law. Moreover, she also obtained a letter of introduction from the head of her department, the Principal Administrative Secretary, Cabinet Office, which all the directors of the national research institutes, with the exception of the universities and the international research institutes, demanded for such an undertaking. On the basis of the employer's authority, the directors of the national institutions wrote letters authorising the researcher to interview their personnel and also assigned a contact person. Thereafter, the researcher met with the contact persons and came up with a tentative timetable for interviewing. This took quite some time, with some institutions taking a month to issue the authority, despite the researcher's frequent visits to the institutions to make a personal appeal.

After obtaining the required clearance, the researcher conducted two pilot interviews in one research institution to establish the kind of responses the interview guideline elicited. Tentative analysis of data from these initial interviews was done to help enrich the interview guidelines for successive interviews. The researcher sent this to her supervisors who recommended further probing. The researcher then revised her interview guidelines and interviewing technique to seek explanation especially on how and why of the reported events. After the revision, full data gathering commenced (See the field research plan attached - appendix A-1). 
It is worthwhile noting that partial data analysis was an ongoing process during the data collection, in order to identify areas where further investigation was required. This resulted in information-rich interview transcripts that helped considerably in drawing out and explaining the participants' world view regarding ICT-enabled research communication.

As outlined in 4.4.1.2, confidentiality was maintained by assigning pseudonyms for all research participants and institutions.

\subsection{Data Analysis Tools and Methods}

The two guiding principles in a qualitative study that assumes an interpretive epistemology stipulate that data analysis is an ongoing process that feeds back into the research design right up to the last moment of data gathering, and that "whatever theory or working hypothesis eventually develops must grow naturally from the data analysis rather than standing to the side as an a priori statement that the data will find to be accurate or wanting” (Powell \& Connaway, 2004, p.196). Data collection in a qualitative study integrates with data analysis. Hence, qualitative research "requires a cyclical approach in which the collection of data affects the analysis of the data which, in turn, affects the gradual formation of theory which, in turn, affects the further collection of data” (Powell \& Connaway, 2004, p.196). The same views are held by Bryman (2004); Creswell (2003); Denzin \& Lincoln (2005); Gorman \& Clayton (2005) and Miles \& Huberman (1994).

This research used qualitative techniques to code and analyse the data collected, according to the emerging themes and categories. These techniques provided rich and thick descriptions of organisational, social, political, cultural and technological settings and events, to generate contextual understanding of the factors affecting adoption of ICT for communication of research in research institutions in Kenya, unlike quantitative researchers who are deliberately unconcerned with rich descriptions which they see as interrupting the process of developing generalisations (Denzin \& Lincoln, 2005). One of the chief reasons for the emphasis on descriptive detail is that it is often precisely this detail that provides the mapping of context in terms of which behaviour is understood. The propensity for description can also be 
interpreted as a manifestation of the naturalism that pervades much qualitative research, because it places a premium on detailed, rich descriptions of social settings (Bryman, 2004).

Specifically, the researcher employed three overlapping processes of qualitative data analysis: data reduction; data display; and conclusion drawing and verification (Huberman \& Miles, 1998; Miles \& Huberman, 1994) (See figure 4.2 below).

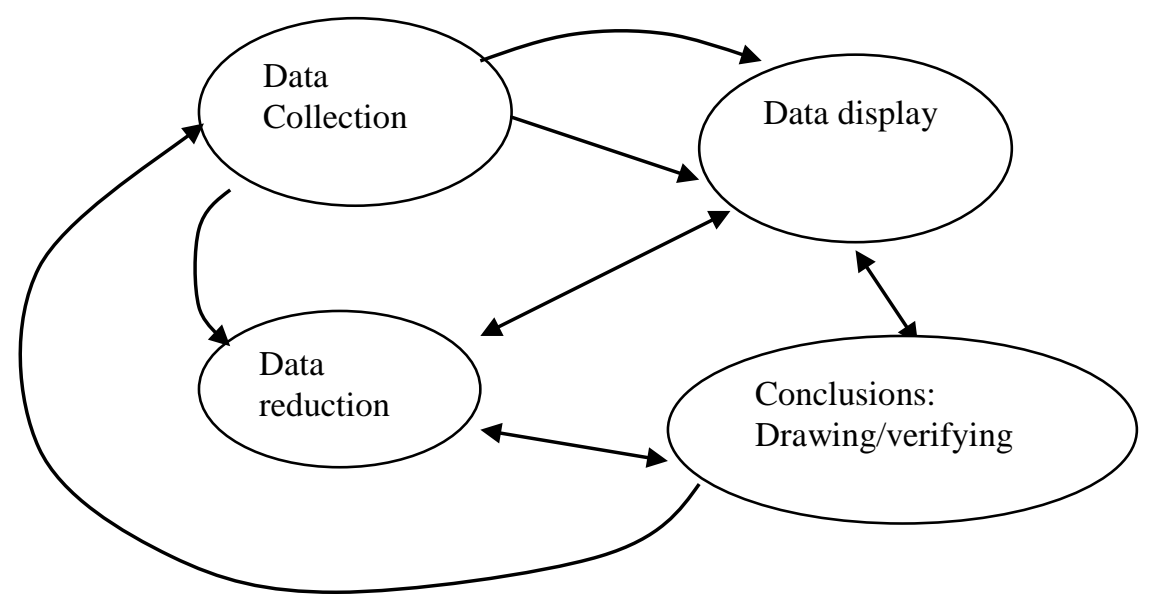

Figure 4.2: Components of data analysis: Interactive Model (Huberman \& Miles, 1998, p. 181)

\subsubsection{Data reduction}

Data reduction is the process whereby one "sharpens, sorts, focuses, discards, and organizes data in such a way that 'final' conclusions can be drawn and verified" (Miles \& Huberman, 1994, p.11). It entails reducing the data into categories, summarising, coding and identifying themes, and enables the researcher to simplify, abstract and transform raw data into meaningful units. Miles and Huberman (1994) propose the following tools and strategies that can aid data reduction as employed in this study's analyses:

- Contact summary sheets: These are documents that contain a number of questions that help the researcher to summarise the main points and capture impressions of any field contact. This enabled the researcher to reflect and focus on the main issues as brought out by that particular contact. 
- Data coding: This is assigning labels to pieces of data, such as words or phrases, which help organise and later retrieve the data. Coding is the process of organising the data into "chunks" before bringing meaning to those “chunks” (Rossman \& Rallies, 1998, in (Creswell, 2003, p.192). It involves taking text data or pictures, segmenting sentences (or paragraphs) or images into categories, and labeling those categories with a term, often based in the actual language of the participants (an in vivo term) (Creswell, 2003, p. 192). This enabled comparison between incidents or events (Strauss \& Corbin, 1990) and also helped in preventing data overload.

- Memoing: Memos are notes that researchers might write concerning coding or concepts. Corbin \& Strauss (1990, p. 198) describe memos as "the written forms of our abstract thinking about data”. They aid in generation of concepts and categories by serving as "reminders about what is meant by the terms being used and provide the building blocks for a certain amount of reflection” (Bryman, 2004, p.405). They help researchers to crystallise ideas while at the same time keeping track of their thinking on various topics (Bryman, 2004). Memoing is, therefore, a conceptual process that aids the process of analytical thinking.

To identify common themes, relationships and potential categories of data in this study, the researcher analysed and coded the interview transcripts by using descriptive keywords and pattern coding based on the elements of the research model and research questions, taking care not to exclude emergent codes. She used the processes of bridging, filling in, extending and surfacing to ensure the codes were updated to take care of emerging issues and themes (Bryman, 2004; Lincoln \& Guba, 1985). Coding was done immediately after the data were collected to appraise with emerging themes and concerns and also to ensure interview questions evolved accordingly to reflect the emerging theoretical ideas and issues.

Soon after codes had been assigned to sufficient data, pattern-matching logic to identify emergent patterns was applied. That is, descriptive and interpretive codes (representing identified themes and issues) were grouped within a series of higher level encompassing themes, identified by a set of pattern codes - explanatory or 
inferential codes (Miles \& Huberman, 1994). The researcher initially used the NVivo version 8, a computer-assisted qualitative data analysis software (CAQDAS), to organise and analyse the qualitative data. NVivo was chosen owing to its ready availability in the researcher's university library and its use in a wide range of research projects (Bryman, 2004; QSR, 2003). The researcher imported all the transcripts into the programme and succeeded in analysing two transcripts. However, she kept losing data because the new NVivo version was still unstable. Therefore, on the advice of her supervisors, she undertook the analysis manually using MS word and spreadsheet. The supervisors also expressed concern that the software would not allow free exploration of the data collected, an opinion shared by Grbich (2007) and the researcher also concurred with.

\subsubsection{Data display}

Data display is the organisation of information in such a way that actions can be taken and conclusions drawn. It makes data more accessible for interpretation and drawing of conclusions. Data display techniques used in this study included text and excel spreadsheets highlighting the various themes and categories, relevant quotes, codes, and notes and memos explaining linkages to and divergence from the initial framework and extant literature. Categories were assigned different colours for differentiation and ease of reference when reporting. The tables helped summarise, synthesise and consolidate information from interviews and served as a reporting tool.

\subsubsection{Drawing conclusions and verifications}

The process of drawing conclusions and verification is an interpretive one, whereby the researcher attempts to draw meaning from the displayed data and validate them. It is about the lessons learnt (Lincoln \& Guba, 1985) from a comparison between the findings and the information gleaned from literature and extant theories (Creswell, 2003). The ultimate goal of this research was to develop a contextual model for ICTenabled research communication by and for scholars and researchers in applied sciences and technology in Kenya. The preliminary model in this research was informed by Rogers' Diffusion of Innovations theory and Hofstede's Cultural Dimensions framework and extant literature. Accordingly, the researcher drew conclusions on points of conformity and divergence and used those to inform the 
development of a contextual model and interpretations calling for action agendas for reform and change in ICT-enabled communication of research outputs by and for Kenyan researchers, as a means towards greater participation in the global knowledge economy.

\subsection{Evaluating Qualitative Research}

Validity and reliability in qualitative research do not carry the same connotations as in quantitative research, owing to the different worldviews of the two paradigms. The positivists' view of reality as something detached from the research participants and researcher contradicts the interpretivist notion of reality as socially constructed and, therefore, inseparable from the views of the researcher and research participants and the research context (Bryman, 2004; Creswell, 2003; Gorman \& Clayton, 2005; Klein \& Myers, 1999; Lincoln \& Guba, 1985; Powell \& Connaway, 2004; Williams, 2000).

In quantitative research, validity is concerned with the integrity of the conclusions generated from a piece of research, whereas reliability concerns itself with ensuring the results of a study are consistent enough to be replicated and a researcher achieves by spelling out his/her procedures in great detail. Bryman (2004) distinguishes four types of validity: measurement - addresses the question of whether a measure that is derived from a concept really does reflect the concept that it is supposed to be denoting; internal validity which concerns with whether a conclusion that incorporates a causal a relationship between two or more variables holds water; external validity - addresses whether findings of a study can be generalised beyond the specific research context; and ecological validity which concerns with applicability of social scientific findings to people’s every day, natural social settings.

On the other hand, the alternative to validity and reliability in qualitative study are trustworthiness and authenticity criteria (Lincoln \& Guba, 1985 and Guba \& Lincoln, 1994). It is these two primary criteria that the researcher applied to evaluate truth values of the findings of this study.

\subsubsection{Trustworthiness}

Denzin and Lincoln (2005) hold that the trustworthiness of qualitative research relates to the value of findings and their authenticity. It addresses the basic question of: "How 
can an inquirer persuade his or her audiences that the research findings of an inquiry are worth paying attention to?” (Lincoln \& Guba, 1985, p.290); whether “you are observing, identifying, or 'measuring' what you say are” (Mason, 1996, p.21). This study used the four criteria of trustworthiness proposed by Lincoln and Guba (1985), which are analogous to the four quantitative research criteria mentioned above, to ensure the value and authenticity of the findings. These included credibility, transferability, dependability and confirmability.

\subsubsection{Credibility}

Credibility is about how believable the findings are and parallels internal validity in quantitative research. The establishment of the credibility of findings entails both ensuring that research is carried out according to canons of good practice which include validation by respondents and triangulation (Bryman, 2004; Creswell, 2003; Gorman \& Clayton, 2005; Guba \& Lincoln, 1994). The researcher in this study applied both principles by first giving the interviewees a summary of what would transpire at the end of each interview to seek their consensus and reaffirmation. Then after transcription, the researcher sent the interview transcripts to the research participants for confirmation. For triangulation, the researcher compared the data collected through the interviews with what was in policy documents, technical and annual reports, and any other relevant documentation. She also paid particular attention to the requirements outlined in the Victoria University of Wellington's Code of Human Ethics (discussed earlier under section 4.4.3.1).

\subsubsection{Transferability}

It is about whether the findings can apply to other contexts and parallels external validity. There are two schools of thought regarding transferabilibility or generalisability of qualitative findings. One school subscribes to the view that it impossible to generalise qualitative findings owing to the fact that qualitative research typically entails the intensive and in-depth study of a small group or individuals sharing certain characteristics, who are not meant to be representative of a population (Bryman, 2004; Lincoln \& Guba, 1985).

Qualitative findings tend to be oriented to the contextual uniqueness and significance of the aspect of the social world being studied and so the researcher cannot predict the 
transferability of findings. Instead, findings of qualitative research can be generalised to theory rather than to populations, what Mitchell (1983) calls "the cogency of theoretical reasoning” (Bryman, 2004, p.392). Thus, it is the quality of theoretical inferences that are drawn out of qualitative data that provide for assessment of transferability (Bryman, 2004; Lincoln \& Guba, 1985). Hence, the researcher's responsibility is "to provide the data base that make transferability judgements possible on the part of potential appliers” (Lincoln \& Guba, p.316) which is achieved through providing thick descriptions or rich accounts of the details of phenomenon being investigated.

The other school of thought holds that in qualitative research there can be moderatum generalisations, where aspects of the focus of enquiry "can be seen to be instances of a broader set of recognizable features” (Bryman, 2004, p.392; Williams, 2000, p. 215). The proponents of this view argue that, when generating findings, the researcher draws comparisons with findings by other researchers relating to comparable groups. They, however, note that moderatum generalisation is limited and somewhat tentative as opposed to the statistical generalisations resulting from probability sampling (Bryman, 2004; Williams, 2000).

The researcher in this study ascribes to the first view that, owing to the limited and purposive nature of sampling, qualitative findings are generalisable not to populations, but to theory. Consequently, she gleaned the qualitative data for its theoretical inferences and ability to provid rich contextual accounts of factors that affected the process of ICT adoption to research communication, as perceived by the participants. Such detailed and rich descriptions make possible transferable judgements on the part of interested parties who can read for themselves and see how they apply to their situation(s).

\subsubsection{Dependability}

This is about the likelihood of the findings applying at other times and parallels reliability. To ensure the results of her research were dependable, the researcher adopted an "auditing” approach which entailed keeping complete records of all phases of the research process - problem formulation, selection of research participants, fieldwork notes, interview transcripts and data analysis decisions in an accessible 
manner (Lincoln \& Guba 1985). She then used peers as auditors during the course of the research and at the end to establish how far proper procedures were being and had been followed. She also constantly assessed the degree to which theoretical inferences could be justified as she moved along (Bryman, 2004).

\subsubsection{Confirmability}

This parallels objectivity and is concerned with ensuring that the researcher can be seen to have acted in good faith by not overtly allowing personal values or theoretical inclinations to sway the conduct of the research or findings deriving from it (Bryman, 2004). The researcher ensured a proper audit trail of the raw data, analysis notes, reconstruction and synthesis products, process notes, personal notes and preliminary developmental information, was kept throughout by working closely with the supervisors and comparing notes with other PhD. students during her analysis and data presentation (Guba \& Lincoln, 1994).

\subsubsection{Authenticity}

There are different types of authenticity that an interpretivist researcher should strive to achieve. First is ensuring fairness (representation of different viewpoints among members of the social setting). Second is ontological authenticity (ensuring research helps members to arrive at a better understanding of their social milieu). Third is educative authenticity which seeks to ensure research helps members to appreciate better the perspectives of other members of their social setting. Catalytic authenticity is fourth and strives to ensure research acts as an impetus to members to engage in action to change their circumstances. Then there is tactical authenticity which seeks to ensure research empowers members to take the steps necessary for engaging in action (Guba \& Lincoln, 1994).

Even though authenticity criteria are said to have certain points of affinity with action research (Bryman, 2004), the researcher in this study tried to achieve certain aspects of authenticity. For example, fairness was achieved through the stratified purposive sampling to ensure different viewpoints were elicited from among members of the social setting. Ontological and educative authenticity were achieved by giving adequate information to the participants about the study and what it hoped to achieve (information sheet) and by member-checking the interview transcripts. This brought 
the participants with the reality of their world as perceived by them. Catalytic and tactical authenticity may have been outside the scope of this research, because the choice to implement the findings and recommendations rested with the government of Kenya and research institutions. However, catalytic and tactical authenticities were the ultimate concern of this research whose major concern was to come up with a contextual framework for ICT-enabled research communication by and for researchers in Kenya as a means to greater visibility of the Kenyan research output and participation in the knowledge economy.

\subsubsection{Klein and Myers' seven principles for interpretive research in IS}

Along with Lincoln and Guba's (1985) criteria for trustworthiness, Klein and Myers (1999) seven principles for interpretive research in IS were also used to ensure checks and balances in the pursuit of quality assurance. Derived from hermeneutics, phenomenology and anthropology, the seven principles are a response to concerns about quality in interpretive field research, and Klein and Myers (1999) applied them to review three published studies. The authors see them as improving the "plausibility and cogency” of interpretive studies (Klein and Myers, 1999, p.79). These include:

\section{The Fundamental Principle of the Hermeneutic Circle}

This principle suggests that all human understanding is achieved by iterating between consideration of the interdependent meaning of parts and the whole that they form. This principle of human understanding is fundamental to all other principles and was the cornerstone of this interpretive study, which required the researcher to engage in a recursive inductive exercise to understand the parts that constituted the whole picture. The inductive study provided elements to understand the parts: researchers, particular Kenyan contexts, their research needs and priorities which informed the whole - contextual framework of factors that affected the diffusion and adoption of ICT for research communication.

To capture the scenario holistically, the researcher interviewed researchers in both national and international research institutions; senior government officials engaged in planning and decision-making processes that affected researchers and how they do their business; and information professionals and science editors that 
worked along with researchers to manage the content or outputs of the research endeavour.

\section{The Principle of contextualisation}

This one requires the researcher to critically reflect on the social and historical background of the research setting, so that the intended audience can see how the current situation under investigation has emerged. Being a public servant and a Kenyan, and having interacted with some of the institutions in her work programme as a programme officer and also during a previous research study, the researcher already had an understanding of the institutional background against which the study was undertaken. Contextualisation was also achieved through analysing the data from individual research participants into specific codes, informed by the participants' experience in their individual organisations.

\section{The Principle of interaction between the researcher and the subjects}

Here, the researcher is to critically reflect on how the research materials (or "data") were socially constructed through the interaction between the researcher(s) and participants. In this case, the researcher was aware her interaction with participants could encourage sharing or withholding of information owing to her position as a government officer in the Ministry of the Office of the President, and in a department that was known for rationalisation of government functions and retrenchment. Indeed, this caused some senior government officers and researchers to demand the researcher produced a letter from her employer indicating that she was really an officer on official study leave, authorised to undertake the specific research. It was only when reassured of the researcher's identity and that her research was purely for academic purposes, not some other agenda, that some participants felt free to share information, despite the valid research permit from the authorised ministry.

On the other hand, some participants saw the researcher's connection with the Office of the President as an opportunity to tell the government what it could do to enhance strategic ICT-enabled research communication. Also, because the researcher was a civil servant, not affiliated to the research institutions, many researchers, science editors and librarians felt able to comment freely on the 
institutional leadership or other limitations that have hampered widespread adoption of ICT for research communication.

\section{The Principle of Abstraction and Generalisation}

The researcher is required to relate the idiographic details revealed by the data interpretation, through the application of principles one and two, to theoretical, general concepts that describe the nature of human understanding and social action. Guided by DOI, Hofstede’s cultural perspectives and gaps identified from the literature, the researcher led participants to share their experience of ICT adoption and use in their institutions and socio-cultural milieu in an attempt to achieve a satisfactory theoretical generalisation (Walsham, 1995). The researcher also took care to incorporate emerging, unique nuances not reflected by the theoretical framework. The intrinsic and iterative nature of inductive thinking, coding procedure and analytical memo writing that is the hallmark of interpretive research, led the researcher to the conceptual understanding of the problem, which could be extended to other settings.

\section{The Principle of Dialogical Reasoning}

This requires the researcher to be sensitive to possible contradictions between the theoretical preconceptions guiding the research design and actual findings ("the story which the data tell”) with subsequent cycles of revision. Hence the researcher forgot the preliminary theoretical framework and immersed herself and interacted with the data from the interview transcripts for possible theoretical insights - points of convergence and divergence from extant theories and literature, as she sought explanations of the emerging patterns from data. This helped minimise prejudices owing to the subjective nature of interpretive study and instead the researcher remained able to identify and incorporate new insights emerging from evidence.

\section{The Principle of Multiple Interpretations}

It requires the researcher to be sensitive to possible differences in interpretations among the participants, which are typically expressed in multiple narratives or stories of the same sequence of events under study. It is similar to multiple witness accounts, where all tell it as they saw it. During the fieldwork, the 
researcher formally interviewed 46 participants, together with an unplanned focus group of 8 in one government ministry, making the total number of interviewees 56. The researcher also interacted and spoke with many others and reviewed documents (reports, brochures, policy documents, strategic plans, other studies, newspapers and institutional websites). These sources at times gave conflicting accounts of some incidents which the researcher turned into opportunities for further interviewing and probing, thus, leading to rich and different perspectives of same issue. This led to deeper understanding of the differing perceptions about ICT-enabled research communications which enabled the researcher to make informed recommendations for policy considerations.

\section{The Principle of Suspicion}

It demands that researchers be sensitive to possible "biases" and systematic "distortions" in the narratives collected from the participants (Klein and Myers, 1999, p.72). This research targeted researchers, senior government officials, librarians and science editors, whom the researcher felt would give complementary information on factors affecting the adoption of ICT for research communication from their own experience and perspectives. The researcher approached them, especially government officials and researchers, with a lot of scepticism about whether they would be willing to share government information, because the majority of them were vetted officers. The researcher also feared they might tell her what they felt she wanted to or should hear, not the real picture on the ground. This awareness made her alert enough to look for nuances of possible distortions and biases. However, the researcher was amazed with the honesty and transparency of the majority of interviewees, especially after they discovered she was a government officer and her research was purely academic. They not only gave frank accounts of what was happening in their institutions but also how they saw their contribution and failure in the whole process of ICT-mediated research communication. It is the researcher's belief that she was able to differentiate the real picture from exaggeration. 


\subsection{Chapter Conclusion}

This chapter has reported how data were collected and analysed, giving justification for the methodology employed. The next four chapters (5-8) present the research findings. 


\section{CHAPTER FIVE}

\section{REINFORCING CAPACITY FOR STRATEGIC RESEARCH COMMUNICATION}

\subsection{Chapter Overview}

Chapter 4 described how data was collected and analysed to answer the research questions in the study. This chapter presents and discusses the first theme - priority scientific research communication needs of researchers and scholars in Kenya - to provide background information on which to base a contextual ICT-enabled research communication framework.

Developing a contextual framework for ICT-mediated research communication calls for the need first to identify and establish the priority research communication needs of researchers in the selected disciplines. Borgman (2007) affirms the necessity for this,

The risks in constructing grand technological infrastructures lie in assuming that "if we build, they will come.”... Scholars do adopt new technologies when they see sufficient advantage in doing so, but they adapt them to their needs. Thus, to determine what infrastructure tools and services will be most useful, it is necessary to understand who the users are and how they conduct their research (p. 149).

There is need for a contextual ICT-mediated scholarly or scientific research communication framework to be guided by the communication needs of the scholars and researchers in the specific environment because "scholarly practices are not consistent, generalisable, or scalable. They tend to be local and idiosyncratic instead, varying by individual, research project, time, and context. The situation calls for ways to balance the local needs of individual scholars, students, and teams with the global requirements of a distributed, multidisciplinary, multi-lingual, multipurpose einfrastructure” (Borgman, 2007, p. 252). Kaniki (2004) attests to the same when he argues that "information needs, even among people, organizations, and countries of the same group or class, are context-dependent,” (p. 83).

The findings reported in this chapter relate to the first two research questions: 
1) What are the research communication needs for researchers in Kenya in agriculture, biology/biotechnology, environmental science and health sciences from the perspective of key participants (that is, the researchers, ministries/departments, research institutions)?

2) What are the main priorities for research communication of researchers in Kenya in agriculture, biology/biotechnology, environmental science and health sciences?

The emerging overarching research communication need was the need to reinforce the capacity for strategic research. This forms the central theme around which the discussion in this chapter centres. The researchers and those in key positions working closely with them in relevant government ministries/departments, science communication and editing, and science information management felt that there was need for government to reinforce researchers' capacity for strategic research. To achieve this, they felt the government should recognise and prioritise research communication through the following ways:

- Prioritising and investing in scientific and technological research communication

- Reinforcing the requirement to do and communicate research

- Providing a mechanism for research communication

- Building skills capacity for research communication

- Recognising and motivating researchers.

\subsection{Prioritising and Investing in Scientific and Technological Research Communication}

All participants interviewed felt that prioritising and investing in research communication were the first priority in the endeavour to leverage strategic research communication. This requires a conducive national environment because "scholarly information practices... do not exist in a vacuum. Social, economic, technical, and political trends all influence the environment for scholarship” (Borgman 2007, p.3). This is an aspect that previous studies in scientific communication ignored (Beveridge, 2004; Kaniki, 2004), but which is an important dimension as a basis for informed developments in scientific communication, even more so as a precursor to ICT-mediated research communication. 
Participants pointed out that prioritising and investing in strategic research communication required visionary political and institutional leadership that understands the importance of science, technology and innovation (STI) research in leveraging a country's development. This was necessary for only then could the leadership enforce research communication by funding it to inform development process and serve as the basis for industrialisation which the government is working to realise by the year 2030 (Government of Kenya, 2007).

However, the findings revealed that in Kenya there was politicisation of research institutions and research tended to be seen as an elitist engagement, leading to marginalisation or relegation of the research community. The findings highlighted the unfavourable political climate in Kenya and other African countries in 1970s through to 1990s, which paralysed research practice in public research institutions. As a research communications official indicated, the political elite “don't see it [research] as research that eventually leads to the transformation of the economic fortunes of a country." Consequently, "there was no investment in this country worth talking about in R\&D as the basis of renewing their performance” (GO1_Ma). Some participants noted that the priority of the political elites was politics and that they were not visionary enough to prioritise knowledge; hence, the issue of research being treated as “tokenism”(GO1_Ma) and not considered in planning and budget allocations:

$\ldots$ it is these government functionaries that allocate these resources... Priority is politics. Priority in Africa is not knowledge or intellectual pursuit... Somehow the African government thinks you can just do it without them [researchers]. There is a token realisation (GO1_Ma)

I think research is always put in the back pan and never taken as a priority issue by our government. The government needs to give more priority to research... appreciate the importance of research... [and] be more supportive of the communication of research (RS7_D).

The dominance of decision-making by the political elites was said to have led to uneven distribution of resources, because funding allocation is made in government. A research communications official commented that those in mainstream government were better facilitated than those in universities and other research institutions due to what he called the "power and the influence of politics and the proximity with where resources are allocated” (GO1_Ma). This confirms Hofstede's (2001) findings that Power Distance tended to be high in societies with centralised decision-making or 
vertical relations. The high Power Distance between the ruling elites and the research and scholarly community in Kenya has adversely affected research communication. This also confirms Uncertainty Avoidance on the part of those in the central government decision-making process keeping the intellectuals of the land at the periphery of the decision-making process and disabling them through lack of facilitation to fulfill their mandates.

The lack of research prioritisation in budget allocations still continues, depriving researchers of much needed resources to undertake any research, as participants across the divide reported:

...in most cases ... much of the strategic plan is not implemented because they have to scramble for resources coming from outside the country from the donors... (RS1_G)

... there is little allocation of money to research that actually reaches the researcher himself, like now I think last year [2006] they had given Kshs. 75 million in the budget but I'm not sure whether any money really got to a researcher anywhere here (LR3_B)

... up to now they have really reduced the university budgets and with the corresponding results that universities have to generate their own income from teaching. This in itself has compromised the possibilities of high-level investments in science and technology. Philosophical foundations have virtually died... there is still a lot to be done to create the enabling environment to invest in science right from the lower levels through universities; the construction of centres of excellence that can themselves allow for high level technological research, high level breeding grounds for knowledge. We don't have them in any of our universities. There is no single proper lab that I know of in all the universities (GO1_Ma).

Consequently, participants said many public institutions were characterised by archaic technology, which means researchers are "not on [the] frontiers of knowledge" (GO1_Ma) and are, therefore, unable to produce research outputs to communicate. This confirms Chakava's (1996) description of research institutions in Kenya, especially the universities, as being "largely politicized, and a majority of university professors are absorbed into the state system. Creativity is stifled ... There is lack of an intellectual culture and debate on important issues of the day.”

Some participants reported that corruption by those in both national and institutional leadership adversely affected research communication in public institutions. They 
indicated there has been misappropriation and redirection of research funds/grants and resources:

... the other power is this aspect probably of thinking research money... is free money. They just think probably we just gonna give this man a hundred thousand (Ksh. 100,000), not knowing that it is worked on a budget. ... I would not be surprised if you found something like that in the universities (LR1_A).

... we wanted to do some work ... at the grassroots and we were manipulated such that we ended up doing it in certain places purely because of the political nature ... Do you know that money was not accounted for to the [donor] and three years down the ground was "shh..." and for a long time X University could not attract grants ... (LR1_A).

Participants reported cases of administrators asking for their cars to be fuelled out of research funds or to be taken along during scientific presentations abroad. A researcher reported that fear of losing their jobs through victimisation by the political elites meant researchers and scholars could only watch their research funds being misappropriated to a level where they could do no research:

$R$. And if you refused to be involved in this [misappropriation]?

P. ... probably you have to look at the dimensions. I personally would refuse because probably I am not going to spend one month looking for a job. If probably I'm going to spend one year looking for a job I might not refuse (LR1_A).

A government official who had been a lecturer commented that lack of prioritisation and investing in R\&D inhibited the development of a research culture in Kenya's research institutions - “it [culture] has not really been engrafted.” The result was a lack of research outputs to either disseminate to those who needed them or to inform the research process:

... agriculture has a very huge potential. It is one of the top sectors in the economy but which has been performing very poorly because ... the benefits of research are not informing agricultural production effectively... (GO1_Ma)

... what research is being disseminated? What? What research? ... there is nobody who is doing that research ... When we did our research in 2005 as to how many researches are going on in this country we were able to kabisa [absolutely] globally collect 405 projects the whole country... (GO2_Mb).

Participants indicated that government's failure to prioritise or fund research resulted in donor-funded research projects whose concern was the donor strategy. A scientist said these projects were fragmented and uncoordinated and did not respond to the government's key strategic issues of reducing developmental challenges and poverty as outlined in key policy documents, Vision 2030 and the preceding ERSWEC, and government ministries’/ departments’ strategic plans: 
Those projects they get from donors are not tackling any part of the strategic plan. They are tackling donor strategy. Donor says I am supporting Africa or Kenya or institute in this area... The scientists are working on independently different projects; so, the message doesn't come together... they are not working in symphony to respond to one particular problem. They are just projects and projects and projects (RS1_G).

Donor-funded research was also reported to have resulted in problems of ownership of research outputs. The funding agencies ended up claiming ownership of the outputs from the little research done and also controlling the extent to which it could be disseminated. This tends to confirm Borgman's view that, "Data resulting from grants made by nongovernmental agencies such as universities and philanthropic foundations do not automatically fall into the public domain,” (Borgman, 2007).

Participants pointed to the need to depoliticise and prioritise research and its communication in government planning and budget allocation to guarantee research outputs that could inform developmental and policy-making processes. This calls for adequate budgetary resources allocations specifically for the research endeavour and communication of the accruing research outputs to inform the same. It also means taking care to allocate resources to improve access to tools and infrastructure and research information dissemination services, as the following excerpts show:

... We must shift priority to science and technology and invest in it because it is the basis of industrialisation ... it is very critical for this government to see what it can actually reap from seriously supporting and leveraging science to inform things like SMEs, ... operations to our industries; knowledges that can monitor and report on development... The issue is about genuine and committed resource allocation to the enterprise of knowledge ... (GO1_Ma)

... Priority is funding so that we can just have research going on so we can be able to know what is communicable (GO2_Mb)

The government needs to ... have the right policies but it must be backed up by ... allocations in the budget (LR3_B).

It is only when the government invests in STI that it can claim ownership of research outputs through intellectual property rights processes like patenting, data ownership and control. Otherwise, as a researcher observed,

“...research must obey the policy of the person who is funding... it is very hard for us to expect to reap where we are not sowing, however little... if the government funds me then the government must demand deliverables from me... But when it is not funding me it has no obligation to seek now all those results; somebody else who is funding you will say no...” 
Thus, prioritising and investing in research and its communication would guarantee results that could be communicated to inform policy and practice and ultimately contribute to the well being of the society.

\subsection{Reinforcing the Requirement to Do and Communicate Research}

The previous section has highlighted how lack of political will or understanding of the role research in development planning and implementation has hampered prioritising and investing in strategic R\&D in Kenya. Participants pointed out that reinforcing the requirement to communicate research outputs required a lot of political good will and support, too.

Participants maintained that strategic research communication called for the establishment of appropriate policies that required researchers to communicate research. They felt this would reinforce and cultivate a culture of communicating research outputs. As the situation was, researchers were not under any obligation to communicate their research outputs. Participants felt this needed to be addressed at three levels: the national politics and culture, government policy regulation and legislation, and the institutional environment.

\subsubsection{National politics and culture}

Of importance is the influence of the national culture in which research institutions exist. They operate in a social and political setting with its own set values and norms, which influence their operations (Makhubela, 2004). This also affects institutional culture, as discussed in 5.2.3.3. The findings in this research showed that what a people value as a nation influences research communication greatly, for example material artifacts, education and respect. This points to a need for cultural change management in Kenya to address those cultural issues that might be a barrier to research communication.

Participants were of the opinion that a vibrant local industry informed by research was critical to sustainable research communication. However, a government official reported that Kenyans seemed to value what was imported more than what was made 
locally in the "jua kali" ${ }^{4}$ sector, and added that they import even what they can get locally “out of the taste to be unique” (GO1_Ma) - to be seen as fashionable, members of the class who imports. A lecturer cum ICT manager in university A confirmed this when he indicated "Kenyans love anything foreign" and "look down upon anything Kenyan.” The participants felt this did not promote innovation and has led to lack of funding to incubate new innovations and local industries that could be supporting research projects/institutions; thus, stifling local research and leading to few or no research outputs that could be communicated.

Another issue that was said to be affecting research communication, especially in the universities, which participants reported has become Kenya's national culture, is the value Kenyans attach to formal education. They explained that special respect is accorded the highly learned who in turn are expected to be experts in almost anything and everything:

...it is a situation that for us even a professor is a professor. In fact if say I'm Dr... in Mathematics, my name is Dr. ... and if somebody falls ill in the place somewhere where I live ..., be sure [he/she] will be brought to me! ... on the basis that I'm learned and I should be able to provide a solution... regardless of specialisation ...It is culture; it is a power distance; it is a respect for authority whatever we interpret that authority to be. So I would attribute these more to our education culture, our educational practice and also that thing of the respect- the respect for power; the respect for people in authority is so ingrained in a country like Kenya... (LR2_A).

The respect for authority based on educational qualifications has created a power distance that has affected research communication, in that it "creates a very big space, the big man syndrome, between these people in authority and the people being served” (LR2_A) to a point of stifling creativity and self-learning/discovery, which is the essence of research:

You can't challenge the lecturer! ... He is the one with knowledge and any book you will ever read it is almost certain that my lecturer has read it. So you see that power distance thing is actually very visible here where I have all the books; I can actually read all the books but if I, for example, find something that is against what the lecturer is saying or may be the lecturer only read one theory and I see three theories and some are conflicting with what the lecturer's said, the tendency is that I'll push aside whatever I've read because "man, the lecturer is right." And I will always depend on the lecturer more than I will depend on my abilities to actually derive that information and knowledge from the material that is available (LR2_A).

\footnotetext{
4 “jua kali” means hot sun. It refers to the informal sector where local entrepreneurs make things for sale in temporary open air spaces using local materials
} 
Participants reported that formal education qualifications were seen as the only thing that matters. The level of academic qualification was said to define who you are and your level of participation in research issues and debates. The more degrees and higher qualifications, the better, for your opinion was given preference no matter how right or wrong you may be. A librarian and government official observed this kind of culture has led to a deep-rooted academic class where those without higher degrees were considered not to have knowledge and, accordingly, were excluded from scholarly discussions and issues.

Participants explained that the academic class made it difficult for highly qualified academics and researchers to be advised on research communication issues by other professionals working with them who had lower qualifications. This kind of attitude was said to have made research communication elitist, occurring only between and among intellectuals; thereby, cutting off scholars and researchers from the society whose problems they are supposed to address. This could not guarantee good research outputs that were responsive to the needs of the people "because they [scholars] are isolated up there and they have missed the realities completely. So they really don't know what is on the ground" (L1_A).

Participants noted that the academic class is deep-rooted and is a national problem because it starts early in school life socialisation where the "teachers-student relationship is that of ivory tower and so it continues upward until you reach university level... Maybe it is right from our bottom the way we were brought up” (L1_A). A research scientist noted that "values come with education, what are our priorities for education ... and basically we [Kenyans] are educated for... white collar jobs. That has been the way it has been for a very long time. So the value is pegged there” (RS3_F). Hence, it was felt that overcoming this culture "is something we have to work on at the national level right from the beginning” (L1_A).

Even among the academics, a lecturer noted that there was a sharp division in relationships owing to qualifications, with the highly qualified being the voices to be heard:

Eh! Eh, eh, eh! Here at the university A... there is a big problem ... I could even call it academic arrogance where may be it will not be the level of age per se (older/newer scholars) but levels of attainment. In any one group you'll have the Misters/Ms, the Doctors and you'll have the professors (LR2_A). 
Thus, the research orientation in Kenya remains elitist. Participants pointed to the need for unlimited interactions by researchers for good research outputs to be realised. This calls for change management to address this attitude so as to create/reinforce a culture that encourages mutual and inclusive research communication.

A participant observed Kenyans inherited the skewed, conservative and elitist value for formal education from their colonial masters, which has persisted while their colonisers have changed from this approach to education to focus more on skill orientation and horizontal relationships. This large power distance in Kenya as a developing country contrasts with lower power distance in the developed countries in the West where relationships between the professor and those with lesser qualifications, including students, is less formal and flat, as a participant illustrated with her son's experience in a university in America:

I remember my own son. He left here after "O" levels and went to the US for university education. The first thing the boy wrote to me was "mum, I'm so shocked that I can actually argue with my professor... I can go to my professor and tell him that he was wrong, his yesterday's theory was wrong” ... (L1_A).

Research orientation in the West, too, has changed from research for curiosity's sake to more strategic and market-oriented research in tune with societal demands (Roosendaal \& Geurts, 1997; Borgman, 2007). This could be a pointer to why there is little or no research communication in developing countries like Kenya where power distance differences seem to prevent free flow of information, unlike in developed western countries like the USA. This indicates the unique scenario of research communication in Kenya and also serves as an illustration of Hofstede's (2001) findings that people in large power distance societies are more comfortable with a larger power differential than those in small power distance societies.

Important in strategic research is also a culture of doing research, which requires continuous and rigorous review of new information to establish research gaps and formulate new research questions. However, some participants reported this seemed to be lacking in Kenya and East Africa. A scientist indicated that people want easy PhDs, while those already with $\mathrm{PhDs}$ do not want to continue doing or communicating research, or even visiting libraries and web sites for research information: 
The difference between East Africa and West Africa as I see is that there are people who want to get big degrees and do these things but they don't want to work for it and those people who have got PhDs they don't want to work after that... The moment they get their PhDs they don't want to do anything. They don't want to research. You see we have our seniors here who have PhDs but they are not interested to go to the website to get information to do research to guide the young ones (RS5_D).

Mechanisms to address such a culture needed to be developed as a means of reinforcing the requirement to communicate research outputs.

Another issue associated with the national culture that affects research communication is what participants described as Kenyans' preference for certain professions like law, medicine, architecture, aviation, which were perceived as prestigious compared to others like being a scientist. Participants felt there was little recognition of scientists or knowledge of what they do. As a scientist commented, there is need to recognise science and research if Kenya is going to enjoy fully the benefits that come with investing in scientific innovations:

...people need to get out of the traditional way of thinking. You will in many instances hear people talking of professionals - be it the politician when they are campaigning or our clergy... they talk of the engineers, the medical doctors, the architect, the teachers, the lawyers and nobody ever talks of the scientists...I think the importance of science should be nurtured from day 1 when people go to school so that they can realise that, besides becoming architects, they can also become scientists or, besides becoming pilots, they can also become scientists. This will enable the nation to give due recognition to science and research (RS7_D).

The fact that the scientific "profession is not glamorous. You don't become rich and famous..." and "because the government does not encourage research" (RS7_D) was reported to have discouraged recruitment of many potential young people into the profession including those who "may be potential Nobel Prize winners" (RS7_D). Hence, there is need for the government to come up with systemic arrangements to make the scientific research profession attractive and as a result reinforce research communication. The poor performance in scientific research in Kenya and many other African countries owing to lack of appropriate mechanisms to support research has been affirmed in the literature (Kigotho, 2008; Kiprotich \& Otieno, 2008; Mwaura, 2008).

The lack of emphasis on science was attributed to ignorance of the political leadership about science and its importance in development:

$R$. Why do you think our politicians don't talk science? 
P. This is because they don't know science. ... You should have some scientists there then they will start talking science. ... They would rather talk about something else rather than science (RP7_D).

Participants said this was reflected even in the awarding of presidential awards and state commendations where scientists were hardly recognised. The awards were said to go to politicians, government functionaries and the non-scientists. This is demotivating to the scientific community and affects the morale of the scientists to do and communicate research. They feel unappreciated for their hard work, as two scientists pointed out:

... we need to have our professionalism... and adhere to that in all our placements, our appreciations, promotions... how many of our scientists have ever got Elder of Burning Spears during national ceremonies? [The awards go] to politicians, government, all kinds of things, athletes. Yes, they do a good job, but where are the scientists? (RS5_E)

... during presidential commendation ceremonies, I cannot remember of any scientists who have received any of these awards. All award recipients I can recall are non-scientists such as the artists. So, I think it starts from the top. Nobody appreciates the area of science...nobody ever recognises what we do... I would like to see such awards given for hard work in research (RS7_D).

Participants felt it was important that the political leadership and government recognise and reward scientists' contributions and come up with schemes/grants to motivate and cultivate scientific talents among the young:

Rather than the Presidential Commendations, the nation could start giving national level prizes once a year to acknowledge these people from the academia and other research institutions and give them awards or have some kind of grants for which the young university entrants could compete (RS7_D).

From the discussion above, it is evident that national culture was a barrier to strategic research communication in that it made it hard to have outputs that could be communicated. There is need, therefore, for continuous awareness creation to minimise research communication barriers that may be created by cultural influence.

\subsubsection{Government policy regulation and legislation}

As well as the need to address political and cultural issues hindering research communication, there is need for clear policy regulations and mandates to demand research be communicated. This study found out that there was no specific policy on research communication/dissemination except for the existence of a broad statement on investing on human capital (Government of Kenya, 2004; 2007). A researcher 
commented communicating research depended on invitation by fellow scientists to do so:

... actually it comes from your peers if somebody knows you are doing something important which they feel should be known by the professional associations which they represent. They will come and ask you whether you can give a talk. There is no actually elaborate ways of communicating with these organisations. It is not structured (LR1_A).

One research participant suggested the need for a clear national policy on research communication, backed by a clear budget, which would link research institutions directly with the planning ministry. He felt that "the whole course of disseminating research, if you were to reel down to specific specialised institutions ... targeting, say, farmers. Research to improve crops, of course, itself is an expensive exercise that requires quite a bit of plan ...” (GO1_Ma).

Participants raised a range of policy issues that should be addressed to facilitate research communication. First was the need for a code of conduct for scientists to guide them, especially in communicating sensitive information, as signaled by a research communications official in one research institute. He commented that what and how to communicate scientific information was left to the discretion of individual researchers who had to be careful what/how they communicated research results that might hurt people by going against cultural norms and practice:

Internally I think it is at the discretion of the individual scientists. Like for example if there is something which may be to a certain community is a taboo to eat, even if you did research and realised the nutritional content in the meat is very high and is eaten elsewhere, you need to be very careful the way you approach that kind of idea. You also have a moral responsibility not to hurt people's spirit...may get into problems. So personal discretion guides how you relate and what to promote (GO3_F).

Leaving it to the scientists' discretion to choose what to communicate might translate into useful research information not being communicated in the process.

Participants also expressed the need for a Science, Technology and Innovation (STI) policy to direct R\&D activities. At the time of the field research, there was no STI policy. The newly formed (2006) Ministry of Science and Technology was in the process of coming up with a draft, Kenya's Science, Technology and Innovation Policy 2007 (Government of Kenya, 2007), but it was yet to be released to the public. The lack of STI policy was said to have resulted in uncoordinated research activities 
and projects whose results could not be consolidated into a whole that could be communicated.

Further, the findings revealed the need for systemic arrangements to facilitate and reinforce research communication that can inform policy and contribute to national economic development. A research communications official noted that, although the government had co-opted scholars and researchers into the senior management positions, "it is not enough to co-opt a few individuals who are educated to government; we are talking about systemic arrangements that renews the whole scholarly and research community”(GO1_Ma). Establishing a working research communication system would in turn recruit and retain scholars and researchers doing research:

... there is not sufficient reinforcement of the requirement for researchers to communicate their message. In other words if you are allocating $\mathrm{x}$ dollars for research, how many of those or what percentage of money do you say has to be allocated for communicating the result? That has to be built in. So if it is not, why is the researcher then expected to communicate? There are no resources set aside for that purpose. So setting aside resources for communicating results is one way to enforce, because the resources cannot be spent for anything else other than communicating results. So that will force the scientist to communicate (RS1_G).

Participants also called for the government to come up with a structure for tapping into talents, people who could be think tanks for the country, and to create an attractive environment to retain them in that:

In the country, there is no structure for tapping into talents, however talented you are. So we have created the impression that only politics can save. So there is nobody really doing the serious thinking that a country needs in the 21st. Century. ...So I would say that my government would not need to just attract individuals to be part of the bureaucratic machine in government in a context in which you are actually an individual in a bureaucracy... the idea of attracting highly educated people and making them civil servants is tokenism that cannot resolve that problem. The issue is about genuine and committed resource allocation to the enterprise of knowledge and to support, to create schemes that can actually make it equally lucrative to be a scholar (GO1_Ma).

Participants felt the government should involve various stakeholders in dissemination of research to facilitate enforcement of the requirement to communicate. For example, they felt the private sector and the mass media, such as the many FM radios and TV, which they said in most cases play music and advertise, could be required to dedicate some air time for constructive activities like disseminating research information: 
But who gives them licenses to operate so carelessly and waste a national resource simply playing music and music? ... I think it is very, very important that if I were licensing a radio station I would say you have to spend at least 60 or $70 \%$ of your airtime doing useful things to the society... But if they spend $90 \%$ music and $10 \%$ some other political stuff, are we really serious about giving a message? So we have tools that are so abused or misused, I don't know what to call them... So if I were in a policy-making situation I'll say I wouldn't give you a license if all you wanna ... do is play music here... People don't live on music. You have to address livelihoods of these people (RS1_G)

I think people in the private sector have a role to play. For example, the safari crew could help in the dissemination of research output (RS7_D).

It also emerged that there was a call for government policy regulation of Intellectual Property Rights (IPR) in Kenya if research communication was to be reinforced effectively. Participants reported that research communication has been negatively affected by unclear government policy regulation regarding IPR to guide ownership of research outputs and patent agreements. They added this has been made more complicated by the fact that the government has not been giving adequate budget allocations for research, as a result of which most research in public research institutions is donor-funded. This was said to have resulted in prohibitive agreements on research ownership and patenting, with the research results going to the funding organisations in line with the saying, "He who pays the piper calls the tune” (RS1_G; LR3_B). This has made it difficult for anyone other than the occasional researcher to get published, as a lecturer explained:

... there are some issues to do with policies of patents, you know, as of who owns what. Some good amount of research that is carried here is actually sponsored by... some foreign organisations. So in most cases the foreign people are the ones who own, the donor is the one who owns the findings after all he is the one who funded that research. So in some cases you find that ... you can't just post them around here because really they are probably patented. Some of these researches by the way before you even start it you sign a document that ... the research findings of these projects are owned by the organisation that is funding you. ... So it is now between the researcher and those organisations that those publications are made and what the researcher gets is just ... that publication... you'd be surprised to go to $C$ to get some very crucial information but it is very hard to get. Probably you might be able to get an abstract because of the patenting agreement that they had... (LR3_B)

... at times where you publish is determined by whoever is sponsoring you (GO3_F).

Therefore, there was need for prioritising and investing in research to facilitate production of institutions' own results/innovations that could freely be disseminated. 
The need for government to plan and adequately fund research to ensure research outputs is also evidenced from a reply by the acting Director KEMRI to the Minister for Medical Services, when challenged that Kenya should be producing own drugs locally. She asked the government to allocate more funds, saying that the Ksh. 3.9 billion allocated for research in 2007/08 was insufficient. She told the Minister that KEMRI had 80 research proposals in just a year but because of lack of funds, they were gathering dust on the shelves. She confirmed that "seventy-five per cent of research funds at KEMRI come from donors. The funds are not sustainable and the government needs to support research through funding" (Limo, 2008).

Arising from the data also was the lack of/ the need for government to reinforce guidelines for research collaboration to govern how research is communicated in order to be able to protect IPR of what is their own:

Some of them even don't go through this system [D's publishing guidelines] and then we find that they have published certain things and we take them to task.... We don't want to restrict anybody from publishing but we must realise that sometimes our collaborators don't care about us in Africa. They just come with some money to do their things (RS5_D).

Borgman (2007) confirms the importance of this when she says, "Determining data ownership is especially problematic for research collaborations that cross legal jurisdictions” (p.174).

The weight of the above findings pertaining to the loss of IPR to research funding agencies and others, due to unawareness and failure on the part of government to allocate adequate funding for research, is confirmed by Mwaura's (2008) statement that "Intellectual property rights are regarded by many people as food for the rich and poison for the poor," (n.p) . In an article entitled, "Kenya still sleeping on its intellectual property rights,” he explains Kenya’s low embrace of IPR, which he says are critical tools in commerce, wealth and knowledge generation in that they protect inventions, scientific discoveries, copyrights, patents, trademarks, industrial designs and in some cases, traditional knowledge and folklore. Whereas the largest number of international patent applications received by the World Intellectual Property Organization (WIPO) from developing countries in 2007 were from South Korea (7 061) and China (5 456) followed by other fast-growing countries like India (686), South Africa (390), Brazil 384), Mexico (173), Malaysia (103), Egypt (41), Saudi 
Arabia (35) and Colombia (31), "Kenya filed only three applications, most probably not because of a dearth of patentable rights, but lack of awareness of what is patentable” (Mwaura 2008). The same is echoed by Kigotho (2008) and Kiprotich and Otieno (2008) who talk about the dearth of scientific research and innovation in Kenya and Africa. Kiprotich and Otieno (2008) quote a speech by a Nairobi lawyer and University of Nairobi lecturer, PLO Lumumba, who describes the universities as "factories for certificates” and “commercial ventures.”

Due to this lack of awareness or clear policies regarding patenting and intellectual property rights, Kenya has lost ownership of its own artefacts that are uniquely associated with its people, and, with that, people have lost their livelihoods. An example is given of the loss of the kiondo [traditional sisal basket] to the Japanese (Mwaura 2008). He adds that the kikoi [colourful piece of woven cotton material, which are exclusively found in the East African coast] could have been lost to a British firm, Kikoy Company, were it not for opposition by Traidcraft Exchange UK, an organisation that champions fair trade in the UK and internationally (Mwaura, 2008). There is, therefore, great need for the Kenyan government to engage in an aggressive campaign to create awareness about the importance of patenting innovations to ensure people protect their intellectual property rights, as Mwaura (2008) affirms:

...the wake-up call has not reached enough people, or the authorities, so as to move effectively to protect not just local inventions and scientific discoveries, but also indigenous knowledge in agricultural products, plant varieties, arts, ritual dress and other cultural artefacts such as the Mount Kenya coffee, the Kikuyu grass, the Meru potato, the Molo lamb, the omena (dagaa), the Machakos honey, the Kisii soapstone, the nyatiti (Luo stringed music instrument) as well as the Maasai akala [footwear made from old tyres and beads] (n.p).

The study also established that there was need for legal reform pertaining to communication of government information. The existing government's Official Secrets Act (OSA) (Cap 187) was regarded as conservative and prohibitive in that it "criminalises any handling of information or sharing it in any way it is possible..." (GO1_Ma). Most government information is treated as official and confidential, which prevents free information sharing:

People have been rather conservative and reluctant to open up and pass on the information. It could be out of the policies which used to be there, the nature of intimidation. There was that element of self-censorship in releasing information even 
to a department which is supposed to relay this information to the outside world (GO3_F).

A research communications official reported that there was no freedom of information because Kenya is yet to enact a Freedom of Information Act. He said the recentlypassed Information Bill (2007) (Government of Kenya 2007) responded to "worldwide trends where everybody is saying we need a Freedom of Information Act” (GO1_Ma) but not issues specific to Kenya. He explained it did not give freedom per excellence and so bureaucratic clearance still prevailed; thus, hampering communication of essential information:

What is amazing to me is that you give this freedom with this hand; by the time you get to the third clause you find it is removed because there is a provision "but subject to the minister doing...” (GO1_Ma).

Therefore, government legislation has become a barrier to information communication and needs to be reformed to reinforce research communication.

Participants commented that the existence of a stiff bureaucracy was a big hindrance to research communication. A government official said that, at times, the bodies mandated to approve research projects did not meet owing to bureaucratic rigours:

... our role here is to coordinate research but we haven't coordinated any for the last 4 years because we have not even been having council. ... The people that approve research have not been sitting. Now the Ministry of Science \& Technology has inherited the problem. Now the problem is we are caught within bureaucracy... (GO2_Mb).

He explained that information had to pass through a chain of officers, some of them very conservative and unwilling to share information, before it could be released to the intended audience. This held up the timely and free flow of information communication:

... then I happen to be this officer that even does not want to communicate or even doesn't know how to communicate... sultanates, you know kingdoms or fiefdoms if you want- people who are not open with information because you think too much information going out is likely to reveal your various behaviour. ... So there is what I call a structural problem within departmental units. Structural in the sense that it's got to come from the boss to go through $\mathrm{X}$, and a $\mathrm{Y}$, and a $\mathrm{P}$ and a $\mathrm{Q}$ before it gets to the necessary site (Go2_Mb)

Decision making process in the university is very much based on government rules and regulations, which is very bureaucratic. The university has itself realised that for it to move it has to cut down on bureaucracy. But how do you cut down on bureaucracy when you are a government institution? So it is very deep rooted. You have to convince (LR2_A). 
Bureaucracy also made government procurement procedures cumbersome. Participants complained that it took a long time to procure research essentials. This conflicts with scientists' style of working, whereby their requirements had to be facilitated immediately they needed something in order to be able to produce results:

The core activity of this institute is research. Definitely research will always get another part - administration, referred to as support staff. ... Scientists are used to, I would say, more or less plan a field trip, pick my gear and the equipment, jump into a vehicle, go [to] camp maybe for 3 days, maybe organise something, while administrators will always follow rules and procedures and regulations. Like you can't just walk in [and say] you want to buy supplies for Ksh.20,000 because you are going to the field. I'll tell you “no it can't work; I have to get quotations; you have to wait” (GO3_F).

This issue needs to be addressed for it slows the research communication endeavour.

Along with the need for systemic structures to facilitate research communication, some researchers reported the need for professionalism in implementing research plans. They indicated there was need to plan appropriately and invest time to execute the plans, as the following excerpt illustrates:

... professionalism. It is not poverty. I think Kenya has adequate resources; we only need to plan better but also spend less time planning and more time executing the plans appropriately, and I stress the word appropriate because Kenya is full of good excellent plans but poorly executed (RS4_E).

The findings pointed to the need for government and other stakeholders to create demand for research outputs, in order to reinforce the requirement to communicate the same. As reasons for there being no research outputs to communicate, participants cited lack of direct demand for research information by those the research outputs are supposed to benefit --farmers and policymakers -- and lack of government enforcement of the requirement for research institutes to communicate. A senior research scientist observed this was not only a problem in Kenya but across Africa:

The biggest problem has been the lack of demand. If I ask you what is the demand for information, can you provide/give me a figure and characterise that demand in terms of priority? ... You can say I want to communicate a message but what do you want to communicate and to who? What is the demand? ...And this demand has to be generated by farmers, has to be generated by policy-makers and has to be enforced on the research institutes. So that lack of pressure is itself a big weakness within Africa. Who is demanding it and what do they really want? ...sometimes I ask can you bring me a list of the institutions and what they demanded or list of farmers and what they wanted so that I can see how to address that ... There is nothing! (RS1_G).

The researcher above went on to explain that the demand for research information was implicit only, as evidenced by the outdated knowledge and skills that farmers use: 
But there is that feeling, and I am part of that feeling, I understand it, it is there that there is a demand because when you go to the farmer and see that the farmer is applying the same knowledge and skills as my grandfather in 1925 you get worried. So they must be dying to have something different, something better (RS1_G).

The lack of demand for research information and outputs could be due to unawareness of what goes on in research institutions. A lecturer indicated most research institutions have no user education component to inform or market their research goods to the public:

... institutions ... may need to sell themselves to the public, you know awareness in itself may be on what can be done. Farmers need to know that they can get some biotechnology-developed bananas in B; you know they need to know that may be somebody can look at mushroom farming in this or that university ... general awareness is low within the wider community ... (LR3_B).

Thus, there is need for research institutions to initiate vigorous awareness campaigns and stakeholders' education fora to let the public know what they do and how/what the public could benefit. This could also provide an opportunity for researchers to get feedback from the public concerning how well their research is meeting their needs, thus, facilitating “cross-pollination of ideas" as one participant put it.

\subsubsection{Institutional environment}

Analysing the institutional environment in which scholars and researchers undertake their research is vital for a clear understanding of their research communication needs and priorities, as a precursor to ICT-mediated research communication. This study revealed many institutional problems and challenges affect the scholarly and research community, thereby, making it hard for them to produce and communicate research outputs. These included institutional mandates, philosophical orientations and policies; institutional leadership; institutional culture; funding and research resources and motivation.

\subsubsection{Institutional mandates, philosophical orientations and policies}

The study found institutional mandates, philosophical orientations and policies to be a major limitation to communication of research outputs. In the first place, researchers commented that the mandate of the research institutes did not require them to communicate research. Their role was to undertake research and leave the dissemination to the relevant government ministries as the following quotes show: 
You see here we are governed by the law and the law says D is mandated to do research on matters related to health and whatever they discover has to be given to those who are mandated to facilitate the application of the findings... (RS2_D)

... We do not have the mandate to carry out extension services and we don't have that capacity...I believe if we were to do it would bring a conflict of interest between us and the Fisheries department (GO3_F)

Thus, the policy mandate regarding research communication in Kenya is problematic, in that it leads to, what a senior research scientist called, "dichotomisation of roles [where] a researcher does research and an extension worker picks this up and relays it to a farmer” (RS4_E). Researchers felt this has led to difficulties in communicating research outputs.

Contrary to the policy mandate that regards involvement of researchers and scholars in dissemination of research outputs as a conflict of interest, researchers expressed the need for them to go beyond just documenting research to communicating research. They felt this was necessary because communication is a two way process between researchers, as the designers of research and custodians of research outputs, and endusers. They felt the research design should have the end-users in mind, which calls for continuous communication between the two ends of the continuum. Consequently, they said, if research communication was to be effective and efficient, the designer, who in this case is the researcher or scholar, needed to be involved in dissemination. Not only would this facilitate the designing of research that was tailored towards communication but also the translation of research outputs into outcomes and impacts:

I would like to say something about policies that these institutions work with. In order to communicate a message that message must have been there in the first place and must have been designed for the purpose of communication to whoever the target audience is. Now it so happens that policies and resources put forth are not primarily designed to develop such a message. You end up with institutions that are there, that are generating the message but the message is not tailored to be either transmitted [to] or used [by] particular clients. So in that context, to give a line example, you end up with research results on the shelf. The reason being the researcher was never perhaps, in the terms of reference of his work, required to communicate [that] message, that result (RS1_G)

The policy mandates researchers to undertake research and leaves the dissemination for the extensionists. This is limiting because a researcher ... should go beyond just doing the research. They should be able to communicate otherwise the outputs cannot be translated into outcomes and into impacts. So need to go beyond just documenting the results of research. We should be able to communicate these results and it should be a dual way. It therefore matters in the design of your research: did you have the end-users in mind? If you had, there should have been a communication right at the start, these people communicating their problems if you are researching on real 
issues. These issues should come from the people and they should have been communicated to you and, at every stage, you should be able to communicate to them (RS4_E).

It is also important for researchers to be involved at the dissemination stage because a lot of tacit knowledge is embedded in data (Borgman, 2007), and may not be effectively passed on by extension workers who were not involved in the research process-conception, design, data gathering, analysis and synthesis. In addition, it was reported the extension workers in Kenya are not adequately facilitated to disseminate research as they lacked tools. Moreover, organising data for communication takes time and skill (Borgman, 2007); hence, there is need for institutions to have researchers on board right from the implementation stage.

In terms of institutional policy for the universities, research communication was reported to be less of a priority than teaching:

Research is not the core of the many of the teaching institutions here. In actual fact the core business is teaching (LR1_A)

The universities are generally mandated to do research, extension and communication of that research but teaching is the main part (RS1_G).

The mandates and philosophical orientations require universities to "do research that is efficiently academic in their considerations to meet certain academic standards" (LR3_B) and does not necessarily have to answer to the needs of the citizen groups like farmers and policymakers. A renowned scholar explained this has led to an attitudinal problem where some, especially the old traditional scientists, see engaging in applied research as vocationalising and trivialising research and taking away their time that should be used in thinking and doing research, not implementing research findings:

I was otherwise recently exposed to a very interesting debate with one of the universities within East Africa where a biotechnology professor was openly saying we do not want to vocationalise education... He said you are making it like a very low level institute whose business is to solve problems. When shall we think and do science for the excellence of science? So there is a philosophical distinction here between the purpose as seen by the professor and the purpose as seen by the government for supporting that institution... (RS1_G).

Consequently, institutions end up with research results that are tailored to be publicised solely through academic channels, such as reputable high impact journals, conferences and any other recognised form of publishing. This view was expressed by 
a whole range of participants including researchers, librarians and government officials:

We are not actually oriented towards that [communicating research]. And I believe it is everywhere ... academics publish the papers; they file them... unless somebody, a donor probably go and actually get a flyer... (LR1_A)

... on the academic side of it I think the scientists are able to publish but ......they [researchers] really look around and be able to publish in these so called high impact journals and to have your work published or even to find the right place to publish, can take some time (L2_E)

The scholarly community in my experience has been conservative particularly in terms of what you might call the channels for sharing because of the orthodox university beliefs and practices "you publish or perish" where publishing was meant to be in a journal ... if you are lucky what they called a peer-refereed journal in some international setting. The other one in the traditional university idea... is that you have learned conferences and workshops... (GO1_Ma).

Publishing is what researchers are mandated to do and so their philosophical inclinations emphasise "publish or perish,” rather than communicating. Consequently, some research products were not useful to the ordinary people and/or conclusive enough to be communicated:

I hate that motto [publish or perish] ... They [scholars] are in panic... We like to encourage people to publish but not rubbish; and we like people to innovate but not for the sake of innovation but because they have found it profitable and useful for Kenyans to innovate. You see like I have been publishing for the last 35 years personally and apart from training one or two PhDs I don't see what my research has done to the ordinary mwananchi [citizen] (GO2_Mb).

So you have material that is shoddy being communicated because of the pressure to communicate (laughs) or publish or perish. So that has been a big challenge and we have been trying to tell our researchers to take time, carry on with their research until when they get conclusive results that is when they will feel free to communicate the same (SE1_C).

Hence, research outputs do not reach the people they should impact on.

Thus, there seemed to be a conflict between government's expectation that research informs developmental goals and research institutions' mandates and philosophical orientations regarding scholarly research communication. Institutions and funding agencies pushed researchers to publish in high impact journals to be evaluated well. This was said to affect dissemination of research outputs to targets like farmers and policymakers, in that researchers are too preoccupied with what/where to publish to have time to think about farmers and policymakers. Consequently, the few research outputs that did exist were in form of journal articles which ended up in international 
journals where few, certainly not farmers or policymakers, could access them. These findings concur with Borgman’s (2007) observations that:

Policies and strategies to share research data have been promulgated for several decades, but the same issues persist. One explanation for this is the lack of enforcement of current policies. Publication requirements are enforced more stringently than are data-sharing requirements. Reviewers of subsequent grant proposals assess the published products of previous research, yet they rarely put much weight on whether data from prior grants were made available (p. 124).

Participants called for policy amendments to allow for more harmonious policy systems where research would be implemented by a team from both research institution and implementing ministry to ensure quality and hasten the process of implementing results:

... we should have either a team at the initial stage from us and the Fisheries department because there is that element of what [findings] you submit there in form of technical report by the time it is synthesised and passed on to the fishermen, it is already changed (GO3_F).

A scientist recommended institutional structures be put in place to help execute existing policies and also for flexibility to facilitate implementation of policies:

How do our communication systems, our agricultural policies relate to other related activities that we undertake or go through? ... policies are just statements of intent. The actual differences come when the statements of intent are translating into realities, into action... What structures are in place or what changes are there in the structure to allow the realisation of the goals or objectives? ... Kenya is blessed that they have lots of policy issues and I think even right now the country is awash with policy changes; everywhere you go policy reviews and so on. But if the form is such that it is rigid it may not allow those changes to be realised or to realise their intended goal (RS4_E).

A lecturer suggested enforcement of performance-based management in research institutions, with promotion be based on products, not publications, would go a long way to reinforce communication of research outputs that can inform the policy and practice.

The influence of institutional mandates and policies on research communication calls for amendments of the same, if effective research communication, traditional or ICTenabled, is ever going to thrive and ensure research information flows to those who need it.

\subsubsection{Institutional leadership}

As well as institutional policies and mandates, the findings established that institutional leadership was a key factor in the research communication process. 
Participants pointed to the need for excellent and visionary institutional leadership in order for research to flourish. They felt research communication needed to be directed by a leader who could think strategically, especially when it came to soliciting for research funding. A renowned scientist commented that this visionary and strategic thinking seemed to be lacking in the majority of the leaders in most research institutions, not only in Kenya but Africa generally. He said many leaders would rather wait for donations, which are not sustainable, even when their institutions are endowed with resources:

I was giving a lecture at the University of Ibadan and I was sad because here you have a research institute which is saying that they don't have any money to carry out research. They want to carry out research on forests... Just one hectare of the forest would produce the US\$60,000 that the director of research is fighting to have from a donor... [However] there is no understanding and no vision to see that if you want the thousands of hectares of forest to be well looked after, cutting off one in order to service the others would be the most logical thing... They say no! No! Don't touch it! Go to a donor and go on your knees and beg for US\$ 60,000 to work out how you are going to look after these. ...it shows that the vision is totally corrupted! Confidence is not there. Thinking is gone! We are not thinking really. So that is it (RS1_G).

Research participants believed visionary leadership was hampered by the stiff bureaucracy, which greatly hindered research communication in public research institutions. They said it hampered free flow of information and communication, leading to unnecessary delays in matters that required urgent attention. They explained this was occasioned by the fact that those in leadership positions were administrators, or even scientists, who in most cases did not see the work of the scientist as a priority or did not understand why it needed to be urgent:

...there is a problem with how the scientific community relates with the administration because the administration guys are from different schools and they don't see the things we think are important as important or the things that we give priority to are not priority issues for them. There lies the conflict, e.g. the administration may be slow in arranging your travelling to collect data even if you inform them of your plans in good time. This is because they don't see the importance of such a research... They even forget that they are here because of the scientists. They pretend as if the institute is an institute of administration, not a medical one (RS7_D).

Moreover, the power distance between the leadership and the researchers put so big a rift between the two groups that they failed to see their roles as complementary. Lack of cordial relationships was reported between the senior and junior researchers where the senior researchers in management positions at times did not pass important 
research information to the juniors. The resulting hierarchical structures caused stiff relationships, making communication between the leadership and researchers difficult:

I tell you, it [communication] is hard [with a lot of emphasis]. We have the hierarchical structures that they inherited from their colonial masters. It will be very hard for the current director to relate with junior people of even their assistant directors... Like if the [institute] director would like to talk to [me], he ... has to go through my boss or he will give the responsibility to my boss and my boss in turn will relate to me... It is not possible for me to go to that office and say whatever I want to say.... if those people above you are given information they won't pass it ... It will never reach the people who are managing the activities. I go there [to the field] and ... I am told "Oh! We are not aware of this!” Or information might have been sent through an email to a centre director-their computers are connected with few megabites through the only telephone line - it will never reach the scientists. ... (RS6_C).

Thus, there was conflict in terms of the focus and interests of the scientists and the leadership resulting in power distance. This barrier to research communication needed to be addressed if research communication was going to be reinforced.

The public research institutions whose research programmes seemed to be doing well are those where leadership cooperated and collaborated with members of staff to give direction in implementation of research programmes. This ensured there were clear mechanisms of communicating so that researchers were kept abreast with what was happening in their field and also had fora where they could share their research outputs, as a scientist explained:

... [our director] is extraordinary. I admire that man. You see he has excellent leadership qualities. He is a very good listener. If you have to be in leadership you must be willing to listen and that is the hallmark of [our director]. And then he has this foresight. That is why we have the African Forum, the journal, the electronic infrastructure which he is building. It is a whole strategy (RS5_D).

Such participants described their institutions as having focused and assertive leadership that allowed an environment that was conducive to both the research community and the funding agencies. The leadership was said to be vigilant and diplomatic enough to ensure donors' assistance was on the institution's terms, in line with their strategic goals:

You see what that man did [the Centre Director in one of the institutes]... he has got all of them [the donors] under control and they have put up a facility for him and are pouring in money... Use your diplomacy and agree with them but let them do it at your terms (RS5_D). 
Other participants reported their CEOs had an open door policy where researchers were free to communicate and be active in research:

... our current CEO has an open policy- anybody, any researcher can make a comment, hold a press media briefing without a recourse for his authority...we have found that this kind of approach is working and our officers are responsive and we have not had an incidence which has got a negative implication either to the institution or the quality and the facts of the information so released (GO3_F).

Overall, the researchers in the international research institutions reported their relationships with leadership were horizontal with a very thin bureaucracy. The scientists in these institutions said they were facilitated, enjoyed doing research, and communication among themselves and with the leadership were efficient and easy. They explained theirs was a multicultural environment with the majority of those in leadership coming from the developed Western societies. So, the culture in these international institutions was Western with very small power distance, as one of the research scientists, who previously had worked in the public institutions, commented:

At $\mathrm{E}$ we maintain a very thin bureaucratic process and we are quite porous. For example, if I feel that I need to communicate some information to the top, say the Director-General of the institute, I can inform him the same time I am informing my boss and it will not be taken in bad taste (RS4_E).

All this suggests leadership is important in research communication, for it provides direction and a facilitating institutional environment. Therefore, institutions need strong leaders.

These findings confirm Hofstede's (2001) assertions in his study on organisational culture that power distance tended to be high in societies with vertical relationships than in societies with flat structures.

\subsubsection{Institutional culture}

In addition to institutional leadership, this study found institutional culture to be a barrier to research communication in some research institutions, which could be attributed to many factors. First, there was a general lack of a research culture in most national research institutions in Kenya resulting from the prevailing national culture regarding research communication (see 5.2.1).

Secondly, within and between the institutions there was the culture of not wanting to share information, resulting from the larger national policy environment which lacked a national code for conduct for researchers (see 5.2.2). 
Thirdly, public servants were reluctant to open up and pass on information, owing to the existence of policies requiring self-censorship like the OSA (see 5.2.2). Participants said this resulted in the culture of rigidity common among and within many public institutions when it came to communicating government-sanctioned information, as a science editor explained:

One of the things you are required to do when you enter some of these places like $\mathrm{C}$ is to sign a secrecy agreement policy so that I'm here bound by an agreement ... and, depending on people's faith, the interpretation of whatever they have been made to swear to ... there are those ones who will actually give a total blackout to anybody. Like you come here for information like you have done and I tell you that is for management, go to my boss R\&D. ... to be able to work and report anything substantial you need to revoke $90 \%$ or largely everything that ...ties you up ... C realises that bodies like $\mathrm{G}$, E [international research institutes] do a lot of collaborative research together but these other bodies are able to move faster because they have open mandates... (SE1_C).

[The researcher experienced the effect of this law in one institute. Despite having a valid research permit and written authority and an introduction letter from her employer and the institute director, a participant still required the researcher to confirm her identity before divulging what he felt was secret information:

P. Eh you told me you are a Kenyan?

R. I'm a civil servant actually.]

Withholding information is another aspect of institutional culture that hinders research communication.

Participants commented that, although the government was moving towards government accountability and transparency in terms of performance based evaluation, as per the recent signing of performance contracts by ministries/government departments, some public research institutions were still slow in making their research outputs known. So unwillingness to communicate still prevailed, although the push for change nationally was bound to change things and make institutional leadership adopt and enforce a more open research communication policy, which would leave researchers free to communicate.

However, a communications official maintained that unwillingness to communicate within the core research institutes arose from, what one research participant saw as, the "group phenomenon", which characterises specific profession groups, and makes them see themselves as belonging to another class: 
Well, scientists will always remain scientists. I mean a bit of dragging, sometimes you are forced to really go out and look for this information. I mean there is that because even somebody announcing a conference forwarding that information is a problem. Scientists are in their own class. There is the perception in the behaviour. I mean a scientist and maybe an economist and a journalist, a teacher maybe -professions-have their own unique characteristic. I think is a group phenomenon (GO3_F).

Another element of institutional culture affecting research communication hinged on traditional beliefs and practices in research and academia that peg promotion on what scholars and researchers have published in renown peer-reviewed journals or learned conferences and workshops. This affected the research outputs communicated, in that they were tailored for those in the particular discipline and, therefore, highly technical. Hence, target groups like farmers and policy makers might be saying there are no research outputs to inform their practice whereas there are, but not tailored for them. The following excerpts illustrate this:

The scholarly community in my experience has been conservative particularly in terms of what you might call the channels for sharing because of the orthodox university beliefs and practices "you publish or perish" where publishing was meant to be in a journal and that is what they considered (GO1_Ma).

They [universities] have that understanding that they are to pursue academic freedom, meaning they are to carry out their work as they deem fit for the academic purpose. And only solve a problem if so it happens ... hardcore scientists who believe in excellence in publishing their work. So the work exits to the publishing houses. ... So it is following a particular pattern (RS1_G).

Unwillingness to communicate could also be explained in terms of the power distance resulting from the bureaucratic structures. Participants in research institutes reported that some scientists in senior management did not communicate important information to those below, as the excerpt shows:

... then I happen to be this officer that even does not want to communicate ... (GO2_Mb)

Our researchers here ... do not believe that they need everybody else. ... researchers feel that they are very powerful and they have no time for ...non-teaching staff and so we don't ask them, and because they don't ask, they don't get information from administrators, very useful information; they don't get information from librarians; they don't get information from the technologists, etc., all of whom are specialists by their own right. ... So to me even when they are doing research, it is half-half: we have not explored all areas to get the right information we want. ... Our teaching staff in the universities is too academic, too academic and I am not ashamed to tell you that they are too academic that they have no impact. They don't have much in practicality... (L1_A). 
The culture of unwillingness to communicate within and across research institutions was also attributed to the nature of scientific research. Participants commented that good research takes time to produce results and so scientists tended to hoard results until they had exhausted everything that could come out of the data:

... it also depends on some discipline because to come up with proper findings which can withstand the test of time you don't go out ... and put that on paper. Good research goes over a period of time (GO3_F).

Borgman (2007) confirms this when she says that scientists will not share research data unless they are sure it has been authenticated and have published and patented what need be.

However, in research institutions, like D and G, where participants reported minimal power distance and in international institutions like $\mathrm{E}, \mathrm{G}$ and $\mathrm{H}$, life was mutually inclusive in that both senior and junior scientists learned from each other, with the senior mentoring the junior in most cases. Relationships were horizontal and communication was free:

...younger scientists are looking at the older ones as their consultants. They check on them to find out how they can do best in their areas. So we consult a lot. ... There is quite a free moving kind of communication between the seniors and the juniors in terms of research (RS2_D).

A scientist reported that "different work ethics and values” between the well-endowed international research institutions and poorly facilitated national institutions hindered research communication in Kenya. The uneven facilitation between researchers on both sides has led to low motivation and different commitment levels for those in the public sector. Participants said researchers in public institutions work under poor conditions and are poorly remunerated, despite being as qualified as those in the international institutions. The resource and remuneration divide between the two is in itself a form of power distance and causes problems along the communication chain; thus, hampering effective research communication, in that both the well-endowed and the poorly endowed had to work together. This resulted in low research impact because, as a scientist in an international institute who had worked in the national system explained, "the ... commitment level for the scientists working at $\mathrm{E}$ [international institute] is not the same one [as] a similar scientist with the same qualifications working in a national system” (RS4_E). The poor work ethic in the public research institutions was said to have led to what one participant termed inertia 
and lack of proactivity among the researchers, necessitating the development of mechanisms for cultural change management to resuscitate research communication in public institutions.

The findings pointed to a need for a policy that requires research be predicated on a clear needs assessment in order to produce results that are relevant to the needs of the community and also to attract funding for further research from interested parties. However, participants noted research in Kenya has not been preceded by needs assessment and the mostly donor-funded research was not likely to provide answers to the problems afflicting Kenyans, because the donors had their own predetermined agenda:

But is there a message, a way of sampling that need and bring it forward? It is not there. It is left to the researcher again to go back and investigate what the farmers' problem is and come back again and do some work to solve it. But it doesn't happen because the donor is not interested in solving that problem... They always have their agenda, their own strategy... (RS1_G).

Needs assessment would help identify gaps that would enable researchers to engage in research to provide answers to them.

From the above discussion, it can be concluded that the lack of, or existence of prohibitive government policy regulations and legislation discouraged strategic research communication. There was need, therefore, for a conducive government regulatory and legislative environment to reinforce the requirement to communicate research to inform policy making and implementation processes. Moreover, issues of institutional environment needed addressing for they also slowed research communication.

\subsection{Providing a Mechanism for Research Communication}

Further to the requirement to do and communicate research discussed under 5.2, strategic research calls for coordinated implementation of research programmes to address the strategic issues of the country's developmental needs as per the policy aspirations. It requires researchers to closely interact with each other and those in the community and to collaborate with industry to be able to innovate and come up with research questions and answers that address the societal problems. However, the research findings identified a gap in the mechanisms for coordinating and 
communicating research outputs and revealed the individualised and uncoordinated way research was conducted in Kenya. Participants reported feeling frustrated with the research environment owing to the lack of policy and communication strategies in many research institutions, which would coordinate implementation of research and ensure the limited research funds were directed towards projects whose results could easily be put together and implemented. Sourcing for research funding and implementing research projects was left to individual researchers’ discretion:

Researchers have generally been frustrated by the fact that they do not do their research programmes in a manner that is cohesive that the whole team is working on one programme and they can reach a point where they can say now this is stuff we can communicate. The scientists are working on independently different projects; so, the message doesn't come together... ... that result is not necessarily very useful in its own way. It needs to be combined with so many other results in order to develop one message (RS1_G)

... for a long time the university did not bother about what you do. It was personal including how you solicit for that money ... All what the university is asking is should you have published put it on the web; let's see it and your C.V. resume... So you become a good researcher depending on your capability (LR1_A).

The lack of research communication policy and strategies could not guarantee results that constitute a whole that can be communicated. Participants pointed to the need for coordinated and organised research communication implementation:

... to reinforce, to reinforce the capacity to continue generating new knowledge in the research work itself in a way that is sufficiently strategic. The scuttled way we are doing things is what is causing the problem. So we must invest in our scientists to work in symphony and for specific programmes that are clearly producing solutions because as long as we are not working to produce solutions that can be brought together, then there is a problem (RS1_G).

Researchers, one citing the case of the Nelson Mandela medical school in South Africa, expressed the need for an institutional policy that would ensure there was a department that monitored the flow of research funding and coordinated implementation of research projects in order to bring together the outputs of individual researchers and institutions:

That is a limitation because if it [university] is not getting involved in that system [research communication], encourage the people to do that and giving the support ... I was telling them a case of one of the institutions we have visited in S. Africa in the Nelson Mandela School of Medicine where actually somebody sits on a computer daily in a whole division just to monitor what grants are coming and actually distribute them to the respective members. We don't have anything like that... we have not yet reached that level (LR1_A) 
In order to bring such results together to form a whole communication unit or complete message to communicate you require that the institution has the culture... the institutions have a mechanism that assembles that message so that it could be communicated (RS1_G).

Having a monitoring body would rid institutions of costly duplications of research projects and in the process help in the redistribution of research resources. Researchers also called for a body or government department that could liaise with researchers and citizens regarding research communication issues. The department could repackage research information into simpler language and formats in readiness for dissemination to those who needed research information and would also act as a point of contact on issues of research communication; thus, freeing the researcher to concentrate on research and also ensuring fast access to research information (see 5.4).

Providing a mechanism for communicating research would also mean establishing ways to link the research institutions with the community it is intended to inform. The research findings revealed there was a disconnect between the knowledge institutions and the planning and policy-making wings of government and practice. A research communication official said this was a common problem, particularly in the universities in East Africa. It resulted in a lack of cross-pollination of ideas where research was not informing policy and practice and vice versa as the following views from a cross section of the participants indicate:

One of the most sobering realities that I have come across is essentially the huge disconnects, throughout E. Africa, between ... the knowledge institutions, especially the universities, and the planning and policy-making wings of governments, ... It seems that knowledge institutions, particularly the traditional universities are operating in a parallel direction from policy-making ...there is no close and crossfertilising engagement between producers of knowledge in whatever field and people whose responsibility it is to turn them into policies and implement them for the benefit of the national planning and national progress. ...They [research institutes] were in fact intended to bridge the gap between research and policy-making. As to whether they have done this effectively it remains a moot point. It is as though we have educational institutions for their own sake. There must be a forum in which science directly informs ... (GO1_Ma)

... although we are employed for three things-teaching, research and information dissemination -that link in-between, i.e. information dissemination to the community, is not well developed... [The] community does not know what happens within research and teaching institutions (LR1_A)

The challenging thing to researchers is ... trying to disseminate; so the people outside there, the common people need to know about it so that they can also check what is happening there. But so far not many of them have got to know. I think general 
awareness is low within the wider community and, because of that lack of link, then what comes from the research to go to the field really doesn't travel that fast (LR3_Mb)

... we are known internationally but the fisherman across there in the boat does not know what we are doing (GO3_F).

Participants gave different reasons for the disconnect in research communication. First was the issue of power relations between those in government and research where government officials did not seem to understand the value and role of research in the planning and decision-making process:

Occasionally you try to involve academics but really the attitude in the government is that we are not looking for scholarship. They will easily tell you this is not an academic exercise. So scholars are seen as theoretical armchair academics engaged in something that is not practical (GO1_Ma)

... they [administrators] think researchers are mad people or they are doing research for the sake of it... there used to be this clique of people that used to imagine that professors are always doing research and this research is not helping the nation (GO2_Mb).

These relational weaknesses at the level of government administration and the research institutions and the local community and the research community need to be bridged, and closer relations among the research community, leadership and local community forged, in order to produce meaningful research that can inform and be informed by the needs of the community.

There was also the issue of outdated policies on which research implementation is based. An example was given of agricultural policies that were not value adding because they are not informed by knowledge from research. This has resulted to what a research communications advisor called "overdependence on rain fed agriculture”traditional methodologies in agriculture which cannot produce results worth communicating, for, as he explained, “you can’t be that traditional and expect to have your green revolution.” A research scientist talked about production-based agriculture where there is no value added through processing, leading to agricultural practices that are not paying and, therefore, not sustainable enough to warrant results for communicating.

Disparities in the communication infrastructure also contributed to the disconnect problem. The well-endowed international research institutes and the national ones that 
enjoyed good donor funding have not found it easy to disseminate research information because their counterparts in national systems and target clients had no means of receiving the information or the innovations. Researchers need a mechanism to communicate research outputs where the extension officers/ research implementing agencies have functional information communication systems if dissemination of research innovations and methodologies is going to succeed, as one scientist recounted:

But communication is a two way process. So we may be well placed to receive the information we want but how do we disseminate the information that we already have or we have generated? Besides we have to work with others...So the impact of our work, our results or whatever information we have cannot be great if we cannot communicate with others who unfortunately don't have the equal access or easier access to the information we generate. ... So the net effect is that it may not be a very, very effective transfer of technology or methodology to the end-user (RS4_E).

Participants also attributed the disconnect to Kenya's curriculum, which, they felt, did not respond to societal demands, nor was in tune with global research trends. The disconnect among the knowledge institutions and the planning and policy-making wings of government, practice, industry and market requirements has resulted in training institutions producing a human resource base with irrelevant and/or outdated skills, as one participant observed:

Structured engagements between products of research in key sectoral areas...are really thin. ... Research institutions have tended to train in a parallel direction ... in this job [research advisor] I meet a lot with people in the private sector who sometimes complain a lot about the absence of right and relevant skills in the market because institutions are not set to meet these needs (GO1_Ma).

Participants underscored the need for government intervention in implementation, coordination, supervision and monitoring of research projects in order to link the various stakeholders - knowledge institutions and private sector institutions such as entrepreneurs, manufacturers, industry, media houses and labour - for effective research communication:

... the keyword we are using there is integration. There has to be a convergence point coordinated by some recognised body... between knowledge and research institutions, government, labour and the private sector...There must be a way in which they dialogue. So it is integration and convergence which is consciously supervised and monitored by government so that they direct it so that people in research know exactly what people in the private sector need (GO1_Ma).

Participants felt this could guarantee the production of results to communicate in the long run and help the government address its key goal of wealth creation as outlined 
in the ERSWEC and Vision 2030 (Government of Kenya 2004; Government of Kenya 2007).

Further, participants felt the gap between the research institutions and the society had resulted in research failing to address indigenous knowledge, owing to the communication breakdown. As discussed earlier, research in Kenya was said to have been elitist, with researchers working with the elite and concentrating on publishing (see 5.2.1 \& 5.2.3.3). Hence, some interviewees expressed the need for a mechanism in which researchers could work closely with the community to address all aspects of life in order to tap into indigenous knowledge in people's minds that could be critical in informing the research process and outputs.

The data revealed that it was vital for researchers and the leadership to have a good understanding of communication, in order for them to come up with effective strategies for communicating research. However, a communications official explained communication as a discipline/practice was not well understood or recognised, with some regarding it as publicity. He said lack of clear understanding of what communication entails had resulted in lack of prioritisation, planning and budgeting. Consequently, there have been no mechanisms put in place to facilitate communication of research outputs (as already discussed under 5.2.2 and 5.2.3.3).

Lack of clear mechanisms for communicating research was said to have resulted in researchers who did not know where they could go for information in the first place. Traditionally, libraries were the outlets for research outputs but, as one librarian reported, “... that bit is not well established; our researchers do not seem to know exactly where to go to look for information: whether to go to the libraries, authors, or subject specialists $-\mathrm{I}$ think that is not very, very clear to them ... communication is not well established...” (L1_A). She blamed this partly on librarians’ failure to have an impact on researchers through marketing and "educating the user that the library has something to offer." She explained that library management was not useroriented but instead tended to focus on protecting information and establishing standards. There has been no focus on the user, user needs and user education- how to convey information to them. This resulted in a scenario where "the poor researchers have information next door and they don't know because the person who is managing 
that information is not marketing it” (L1_A). Hence, researchers did not appreciate libraries as sources of information and "as a result they do not resort to libraries except in cases whereby they have been told there is nowhere else but the library but, if they can avoid the library, most of them do" (L1_A).

The lack of a clear mechanism for research dissemination was evidenced especially in the universities where it was left to the discretion of the researchers to deposit publications with libraries. Participants said the publications might never get to the library where they could be accessed. Researchers also reported of cases where they co-published with others in international institutions but the research findings never became known locally due to lack of mechanisms by institutions to make research outputs available. Hence, you had a scenario where there could be a large number of publications by researchers in research institutions in Kenya but they were not attributed to them, leading to invisibility of their research outputs:

That there was a lot of things that were in hard copies but they were not actually being seen in the other communities and even if they were being seen they were not even being associated with University A. Like you would find publications from University A that are published with somebody else in US and this would not be reflected as a university publication... (LR1_A).

A lecturer explained that this lack of mechanisms to make Kenyan research visible and easily accessible disconnected researchers from their fellows within their own department, other research institutions and the wider community:

... you find many of us have got a lot of papers of course for communication purposes... In terms of disseminating within the university you may find that you are in this department and somebody else in another department don't know what I'm actually doing because I have to take a hard copy to library and you can now imagine from there whether actually the community is going to know what I'm actually working on ... (LR1_A).

Participants proposed mechanisms that could help disseminate research outputs such as utilising existing professional and scientific networks, promoting African scientific information fora, establishing of research communication offices, individual donor initiatives, consortia models and setting up model schools and centres of excellence.

\subsubsection{Research Dissemination Mechanisms}

Professional and scientific networks, whether local, regional or international, were suggested as one way of facilitating researchers to communicate research outputs. However, few of the participants interviewed in this study had access to these. 
Moreover, the professional and scientific networks seemed only to provide a link between and among scientists and not grassroots communities. Interviewees felt increased institutional and individual participation in these networks would enable researchers to contribute to knowledge and learn from others' work.

Research participants expressed the opinion that fora for African scientists to share their findings could help promote dissemination of research outputs from Africa. These would include strengthening existing communication channels such as journals, conferences and other science fora. Scientists maintained that African fora would help overcome the bias Africans might be experiencing in trying to publish in international journals and would promote lesser known areas of African science such as marine biology, in the same way the African Forum for Health Sciences (AFHES) was doing for health sciences:

Yearly we have the African Health Science Congress, which is a conference that is held by African institutions ... They kind of try to bring together African scientists and whatever the journals they publish are also circulated quite well among the African countries. This exposes the published work to the other African scientists for, like I told you, because of you being an African or probably being that there isn't any name that they [international journals] know, Africans may find it difficult to publish their work... (RS2_D)

... publishing in African journals gets a field like marine, which is very little known, to African audience because may be I think marine issues for the African audience have not been articulated as well as let's say agricultural issues, forestry issues, land issues... (RS3_F).

The findings also suggested that having a research communications office in research institutions to serve as a link between the research community and the public could provide access to research findings. One that had just been introduced was helping "to synthesise research findings either in form of scientific papers, technical reports and tone them down to a level where they can make sense to our consumers” (GO3_F). Italso disseminated research outputs through media releases/coverage, hard research findings, meetings and workshops, feature articles and related issues.

A lecturer noted that some individual initiatives by donor organisations were also helping provide a mechanism for availing research information. He gave the example of the African Institute of Capacity Development (AICAD), a JICA-funded institute, which has built a database system called Poverty Alleviation Information Knowledge System (PAIKS) in one of the universities. PAIKS is meant to be a poverty alleviation 
intervention in East Africa, aimed at capturing and providing research information, especially agricultural information, by placing it in some kind of a central location that is accessible to researchers.

Further, establishing consortia was seen as a viable option for communicating research, which could serve scientists right through to the community level. A scientist cautioned it was not enough for the researchers to talk of their research targets as broad groups, say like farmers; they needed to reorganise and work with consortia so that research communication would be tailored towards the needs of a particular group:

... one of the challenge and it is an important challenge is who is your target as a researcher? The researcher has no target. His target is just farmers. Which ones? Where? We need to form these types of groups (consortia) with sufficient expertise to communicate so that farmers are organised in particular groups or there are cooperatives, unions call them anything... The challenge is that I don't see/ there is no bridge; there is a big gap. So who bridges the message from the research institutes or researcher to the farmer? ... (RS1_G).

He explained these consortia could be liaising with scientists and research institutions and picking up information/messages, interpreting them and using them across the group; thus, they would serve as providers of information. The participants identified a number of professional and farmer groups' consortia that were working very well in facilitating research communication, not only in Kenya but also in the East African region. A "consortium of flower farmers [around Nairobi] are just using one entomologist and it is working” (RS1_G).

Research participants also pointed to information professionals' consortia that were doing quite well in facilitating access to information by the research community, for example, the Kenya Libraries and Information Services Consortium (KLISC). KLISC started in 2004 after the INASP/PERI (International Network for the Availability of Scientific Publications/Programme for Enhancement of Research Initiatives) stopped funding Kenyan access to free online journals, and it was said to be very successful. It comprises members from the public and private universities and research institutes, tertiary colleges, NGO, Public libraries, archives and museums. It is a local initiative that operates on own budget, resourced through membership contributions. Through KLISC, member institutions collectively subscribe to e-resources, something that participants said had been unimaginable before. 
There were also regional and international information consortia and networks that provided access to information to researchers and scholars in Kenya, such as the Association for Strengthening Agricultural Research in Eastern and Central Africa (ASARECA). And the Consultative Group on International Agricultural Research (CGIAR). The ASARECA is a regional network whose mission is to enhance regional collective action in agricultural research for development, extension and agricultural training and education to promote economic growth, fight poverty, eradicate hunger and enhance sustainable use of resources (http://www.asareca.org/).

CGIAR is a partnership of 25 developing and 22 industrialised countries, four private foundations, and 13 regional and international organisations, with a vision of achieving sustainable food security and reducing poverty in developing countries through scientific research and research-related activities in the fields of agriculture, forestry, fisheries, policy, and environment (www.cgiar.org). A library manager commented on the particular value of the CGIAR Virtual Library - a gateway facilitating electronic access to all CGIAR libraries and journals - which was completed in 2006 and is recognised as the best research support group across Consultative Groups (CGs). The CGs' libraries have formed an information consortium called the Consultative Group of Library and Information Services Consortium (CGLISC) and KLISC borrows from this model. Like the KLISC, the CGLISC accumulated research information for access through the Virtual Library and enabled member organisations to collectively bargain for cheap subscription to journals; thus, making it possible for the researchers in the region to access a wide variety of information cheaply (L1_A; L2_E).

Other networks reported to have helped national institutions to access research published in high impact journals were the INASP/PERI; AJOL; Health InterNetwork Access to Research Initiative (HINARI), a programme set up by WHO together with major publishers to provide developing countries with access to one of the world's largest collections of biomedical and health literature; and Access to Global Online Research in Agriculture (AGORA), a set up by the Food and Agriculture Organization of the UN (FAO) together with major publishers to give developing countries access to an outstanding digital library collection in the fields of food, agriculture, environmental science and related social sciences. These programmes were reported 
to be providing excellent service, although the challenges of insufficient facilities, infrastructure, computers, reliable Internet and small bandwidth limited maximum utilisation of the resources. The literature affirms this scenario (Ondari-Okemwa, 2002; Smart, 2003; Beveridge, 2004; Durrant, 2004; Kaduda, Olola et al., 2005; Rosenberg, 2005; Ouya, 2006; Shibanda, 2006; Osanjo, 2009).

The success of KLISC and other regional consortia further stresses the need for local initiatives in ensuring sustainable research communication, which in turn requires local research communities to express confidence and belief in their own ability to come up with mechanisms and solutions to address communication issues in Kenya and Africa in general.

Some research participants expressed the need for Kenya, and Africa as a whole, to build more experts through establishing specialised units of research, which one research participant called "model schools and centres of excellence that really engage and interact with industries, that really inform policymaking” (GO1_Ma). They felt this would serve as a means for developing and communicating research outputs by concentrating the limited research resources into centres. This in turn would leverage scientific communication. The blurb of the proceedings of an international symposium on Open Access and the Public Domain in Digital Data and Information for Science (Esanu \& Uhlir, 2004) confirms the importance of national centres of excellence: "The nation turns to the national Academies - National Academy of Sciences, National Academy of Engineering, Institute of Medicine, and National Research Council - for independent, objective advice on issues that affect people’s lives worldwide” (n.p).

Overall, the need for local mechanisms to facilitate undertaking and communication of research is of the essence, if Kenyan research is to become accessible to those who need it and Kenya's visibility in the knowledge arena to be guaranteed. The findings point out that it is crucial for donor funding agencies, the government and local research communities to go a step further to ensure access to information, because the crux of research information lies in making it available to those who need it. The findings confirm Roosendal and Geurts' (1997) assertion that research information dissemination and use are a condition for strategic research in that, 
... the added value is not anymore in information proper but in its effective and efficient communication, or knowledge management. ... [because] just as societal conditions have been seen to translate into research conditions, strategic research conditions translate into conditions for scientific communication (p. 10).

Thus, ensuring there are mechanisms to communicate research outputs should be a hallmark in research communication.

\subsection{Building Skills Capacity for Research Communication}

In addition to mechanisms to facilitate research communication, the findings pointed to the need for communication skills. A range of participants commented that researchers and scholars were poor communicators who lacked training:

... there could still be a problem here and there in terms of being able to communicate the research outputs ... lack of adequate communication skills (L1_A)

... then I happen to be this officer that even ... doesn't know how to communicate (GO2_Mb)

In some cases some knowledgeable people are very poor communicators. Highly skilled and trained people are invariably very poor in teaching and communication... (GO1_Ma)

... capacity for that researcher to communicate...Not everybody is made into communicator .... We tend to assume that anybody doing academic work, whether you are teaching or doing research, you are automatically able to communicate the message particularly to the uninitiated in terms of understanding science... That is not as strong within our national institutions (RS1_G)

... academics are not trained to talk to the community ... in a language they understand ... (LR1_A)

... African needs to be skilled ... how you communicate if it is writing or if it is speaking... Are the African scientists skilled? ... And how many scientists can say take that message, whatever message it is for their work? (RS3_F).

In addition to language challenges posed by the fact that scientific research is undertaken and communicated in English, a foreign language difficult to master, participants indicated that many African scientists lack writing skills:

One reason an African would fail to write is because we don't write well and you send this to somebody who is fluent in English... he won't get past the first sentence and then sometimes you say "oh because I was an African" but many times it is because we were not able to put the idea across well enough ... colleges do not train us to write... (RS3_F)

Hence, there was a need to train scientists how to write, as an editor explained: 
We are trying also to improve the technical quality by thinking of ways and means of carrying out scientific writing courses for our scientists ... to improve scientist to scientist communication in the region (SE1_C).

The data also exposed the need for verbal/oral presentation skills to address accent problems and sharpen researchers' ability to convey the message in the most efficient way possible:

Even the delivery skills like in the case of presentation skills including verbal communication. Most scientists are actually engrossed in their work in labs and some of them require verbal communication skills (L1_E)

... also presentation. You could go to a conference and still not be able to give your message... people couldn't understand may be your accent... As a scientist how have you presented your work? You have only 15 minutes to give your message. Did you just talk about the methods in all those 15 minutes and did not give even results then you walked away as an African and said people didn't like you? (RS3_F).

Participants said the government's inability to allocate budgets for capacity building has exacerbated these problems.

The study also identified a need to train and expose researchers to intellectual property rights to make them aware of the value of their research outputs and how to handle them so that they did not lose out on their discoveries:

... training scientists... on intellectual property rights so that even as they communicate they know that what they are communicating is information that generates the technologies that are sold as products... they should communicate within the parameters provided for by the intellectual property agreements that are in place...(SE1_C).

A scientist reported that researchers' capacity to communicate was also hampered by fear of criticism, especially in collaborative research with people from other cultures:

... Europeans might not be quick to criticise or to help; they will keep away. So one might fear the criticism but it might not be given to you openly... the people giving critique might not be open to give it in a way that you can use it or you might not be open (RS3_F).

The scientist continued that the situation was complicated by the rigid nature of African scientists, who might be unwilling to communicate and so would not benefit from peer-review:

As an African scientist you might think this is good but you might not be open to get a review from your peers (RS3_F).

The findings point to the need to nurture an atmosphere where open communication can flourish. This would allow evaluation and validation of research output in a bid to 
enhance the quality. This underscores the need for good mentoring as well. In addition to building the communication capacity of the researchers, research librarians participating in the study expressed the need to be also trained on the technical subject matter as well the scientific content. They felt this would build their capacity to assist researchers well.

Participants also pointed to the need for specialised information management skills like information communication and repackaging. The language, format and design of research outputs in relation to target audiences were a big challenge to research communication. Participants reported that it was difficult for research outputs to have the desired impact because of these factors, as a librarian and a scientist explained:

... the gap I see between this sort of high-level kind of research and what the farmer at the grassroots needs to alleviate the poverty is that there is actually a communication gap to be able to communicate the outputs and repackage them such that they will have impact on these targets. ... The kind of research that is also churned out of here should also reach the policy maker because these are the people who will make a difference in the rural area through the policy they make. Again I see a need in addressing the gap between these technical outputs and the impacts it has on the policy makers so that they can make the right policies ... (L2_E).

I have seen many scientists stand there and totally disabled to communicate to a farmer if you told them you shouldn't use any technical language. In my country, Tanzania, we had a case where one time the president, Nyerere, told us we had to go for a meeting where he was opening and since I am going to be here for a whole day and I don't want to hear single scientific term nor terminology and I don't want to hear anything in English. You are going to talk to me in Kiswahili and talk to me so that I understand even if you are talking very complicated terms. But I want to hear it in Kiswahili and without any hard terminology. It was painful (RS1_G).

Moreover, scientific research outputs are in text, yet, some of the users and intended audience are semi- or illiterate.

There was need, therefore, to synthesise scientific research outputs into simpler language that could easily more be understood by non-scientific audiences and further translate the simplified versions into the various vernaculars for the majority at the grassroots. However, such access would not be cheap:

... the fact that you can publish at the top notch journals is good and I would encourage it any time. But packaging this information in something else... in a language that would be beneficial to those farmers (RS4_E)

You have to translate the brochures and booklets into different vernaculars, which is also a cost (RS6_C) 
... you find that most of the research output is quite technical and is targeted to other people who may not understand the technical language. So the aspect of repackaging information is important (L2_E).

Further, some participants expressed the need to repackage scientific research outputs into alternative formats, like pictures, photographs and diagrams, which could easily be discerned by non-scientific and even illiterate audiences, if there were to be leverage in using scientific communication to inform policy and decision-making and contribute to the development process as per ERS 2003-2007 and Vision 2030:

... form. I believe in what I call pictures or reality says more than a thousand words. Most of the information available out there is in texts and there is very few diagrammatic pictorial forms which may make people whom we want to influence to understand quicker or better. Our end-users could be semi-illiterate or not as literate as we are. We need to communicate to them in forms that are easier to grasppictures, diagrams, photographs ... (RS4_E)

... there are no versions which somebody shortens and makes them suitable for combinative dissemination. ... unless people/journalists now come and fine tune this it is very difficult for us to communicate it to the community (LR1_A)

Even the booklets are for the advanced farmers, because most of them cannot read, e.g. those in Moyale, Marsabit, Samburu where 95\% of farmers are illiterate (RS6_C).

Thus, repackaging research information was a need that must be met for effective dissemination. Participants recommended the establishment of an independent body to coordinate research information communication and repackaging:

How do we package information in a way that many multiple users can still get it in the form we want received? This requires a packaging component and the person packaging may not be the person doing the job- you cannot be a jack-of-all-trades. So synergies and coordination is heavily required (RS4_E).

They felt leaving research information dissemination and repackaging to the researchers and scholars would be an onerous job, for they do not have the skills and time to do that. Hence, setting up a specialised body would ensure fast access to information and spare researchers' time to continue doing research to generate new ideas:

...packaging also includes information management skills...most of them don't have skills in repackaging information as it relates to the various target audiences... (L2_E)

... training, empowering us to be able to package our materials because it is one thing to say yes we want to make value use documents. But a value use document needs to be written in a certain way and may be not all scientists can write in such a way.... (RS3_F). 
You also need management of this information because it is not right if you have a body of information and everyone wants to access them at will at their own time. Then you will not have time to generate new information as a researcher; you will be busy communicating only what you knew last year. So there is need for management of this information. Who else? Where can we put x information? Who packages it? ... Otherwise a lot of time will be spent communicating and less time will be spent generating [a] new body of information and in science that is the death of you (RS4_E)

... we have to evolve a system for managers [research information] in institutions ... (RS5_D).

Such a body could repackage research information into languages and formats for a range of audiences, so that it could be applied appropriately.

The international research institutions, and a few national institutions like $\mathrm{C}$, were already repackaging their research outputs into simpler formats, such as brochures, flyers and technical notes, and distributing them to other research institutions and farmers during field days and national agricultural shows. F was also in the process of doing so. However, for the majority of national institutions, repackaging was hampered by inadequate funding predicated on donor support:

... we ask these people to package the information in the form that is usable and we have been publishing a series of the same ... ...but you can't rule out the fact that in some technical note series the communication is scientist to scientist. But largely the communication in [the] technical notes series is from the experts to the user (SE1_C)

... we regularly produce a number of brochures and flyers when funding allows... (SE2_D).

The need for repackaging of scientific output affirms Borgman (2007) when she says, "Nevertheless, making content that was created for one audience useful to another is a complex problem. Each field has its own vocabulary, data and structures...Different descriptions, tools, and services may be required to share content between audiences,” (p.10).

The need to build the communication capacity of scientists has been supported by the literature and is not only a Kenyan/African problem, but global (Alo, 2003; Ventola, 2007; Adewuyi, 2008; Gewin, 2009). Hence, governments and research institutions need to pay attention to upskilling researchers, in order to leverage research communication, whether by traditional methods or ICT-enabled. 


\subsection{Recognising and Motivating Researchers}

As discussed in the preceding sections, research in Kenya has been marginalised for decades, with no investment to keep the research environment thriving. It was reported researchers depended on limited donor funding; they "scramble for projects" (RS1_G) from donors and so "the people who are scrambling for projects once they achieve one the next thing is to scramble for the next one and not communicate that message because their survival depends on having a project” (RS1_G).

In addition, researchers, and especially the teaching community, reported having too great a workload to afford time to write proposals for research grants. Added to this is the lack recognition of what the scientists and scholarly community do, as a result of which “... a number of us are not actually motivated ...” (LR1_A). As reported under 5.2.1 above, state awards and commendations go to the ruling political elite. Further, scientists and scholars remain some of the most poorly paid, despite their high academic credentials and contribution to the well-being of the society, as reported by some participants:

You know they are amongst the poorest paid still in this country. It is impossible to be a scholar (GO1_Ma)

Currently we know, just to give you an analogy, Kenya's political landscape is much more active than any other place because it is well paying now. On average a Member of Parliament gets close to a Ksh. 1 million a month; yet, a professor who went to a great length to be what they are gets a hundredth of that. So these motivational factors are issues which are challenging which need to be addressed if we have to bring to the fore the key sectors like agriculture, health and so on. ... Professors need to be well remunerated. A student leaves the hands of a professor and immediately earns may be three times the professor easily; what has changed? ... So these are issues which may not make professionals go a mile longer to narrow the communication gap or to do something more to communicate the results and disseminate them (RS4_E).

A research communications official said that, unlike in the core civil service, especially at the headquarters, where officers have inbuilt incentives like honoraria and all sorts of training, and facilities like computers to make work easier, the research community were not motivated in any way:

In my office here there is nothing I want that I can’t get; it shocks me. Since I came here [to the civil service from the university] this is my second laptop. I can even say this one is old and I will be given another one. [In my position] they facilitate me to the core. They even give me airtime to make sure that I can call. But at the university, nothing! These guys make sure that I have access to any magazine that I want. In fact the problem I have now is to cope up with reading. So that is facilitation to keep me abreast. That is something a professor at the university also needs...These guys [ministry officials] even gave me a [mobile] phone I never even asked [for] ... When 
people do something which really is their line of duty, they get something... Even university VCs don't get the support we get here! ... You can't do research; you can't write a paper because you are basically trying to survive. I suspect it is better to be a senior civil servant... if you want to survive. You see since I came here I have been able to produce that book! Because I'm effectively facilitated (GO1_Ma).

This lack of support does not encourage university researchers to communicate research outputs (see 5.2.3.3).

Equally demoralising to the research community has been the uncoordinated and individualised manner in which research is done. The lack of teamwork has made it difficult for the research community to realise the outputs that could impact on the communities they are meant to serve. There has been no mechanism for communicating research making it hard for researchers to reach to one another and consolidate their efforts for better outputs (see 5.3).

The demoralising environment has made researchers unwilling to engage in research communication, as a research communication official declared:

All the great scholars and academics in this country have virtually abandoned it. That is why it became easy to co-opt them in government. You know in some countries if they have discovered you are too clever they even detain you. Even the US if you are a genius you have to be detained and given so that you think. Even Korea did that to its geniuses. I can name for you over ten people who have died in misery in this country whose brains, I think, if they were nurtured it would have contributed greatly. That is at the level of human capital which is rare. The late Prof. Tom Odhiambo [founder of the International Centre for Insect Physiology and Ecology- ICIPE] was internationally clever but he died a miserable man (GO1_Ma).

There was need for the government to come up with a clear structure that recognised and rewarded scientists to keep them motivated to continue researching. This also meant paying them well to avoid loss of morale within the research community, and stimulating them to think innovatively and collaborate with industry to generate relevant research products to communicate:

So what we need to do is to create an environment where people can think. Yes. Pay them well! ... So you pay them well and when you pay them well then you will create an environment for thinking. When you create an environment for thinking this is when you get useful research coming on and when you get your products of research, this is when you can innovate and incubate those innovations so to speak and this when now those innovations can see the light of day through collaboration between researchers, research institutions and industry (GO2_Mb). 
Thus, motivation is an indispensable ingredient of scholarly and scientific communication and also plays a key role in ICT-enabled research communication (see chapter 8.2).

This chapter has extrapolated the research communication needs of researchers in the applied sciences in Kenya, emanating from their broad socio-cultural, political and institutional environments. Overall, the emerging priority research communication need was reinforcement of capacity for strategic research by recognising and prioritising research communication. Having discussed the research communication needs and priorities of researchers, which answered RQs 1\&2, the next chapters will address the barriers and enablers to ICT-enabled research communication, which this chapter confirmed was a prerequisite to reinforcing capacity for strategic research. This will be in response to RQs $3 \& 4$ and will in turn pave the way for the development of a contextual framework for ICT adoption for research communication by researchers in research institutions in Kenya. 


\section{CHAPTER SIX}

\section{THE SOCIO-CULTURAL ENVIRONMENT IN ICT- MEDIATED RESEARCH COMMUNICATION IN KENYA}

"New innovations in scholarly communication are more likely to be successful if they work with, rather than against, the social aspect of the system," (Borgman 2007, p. 74)

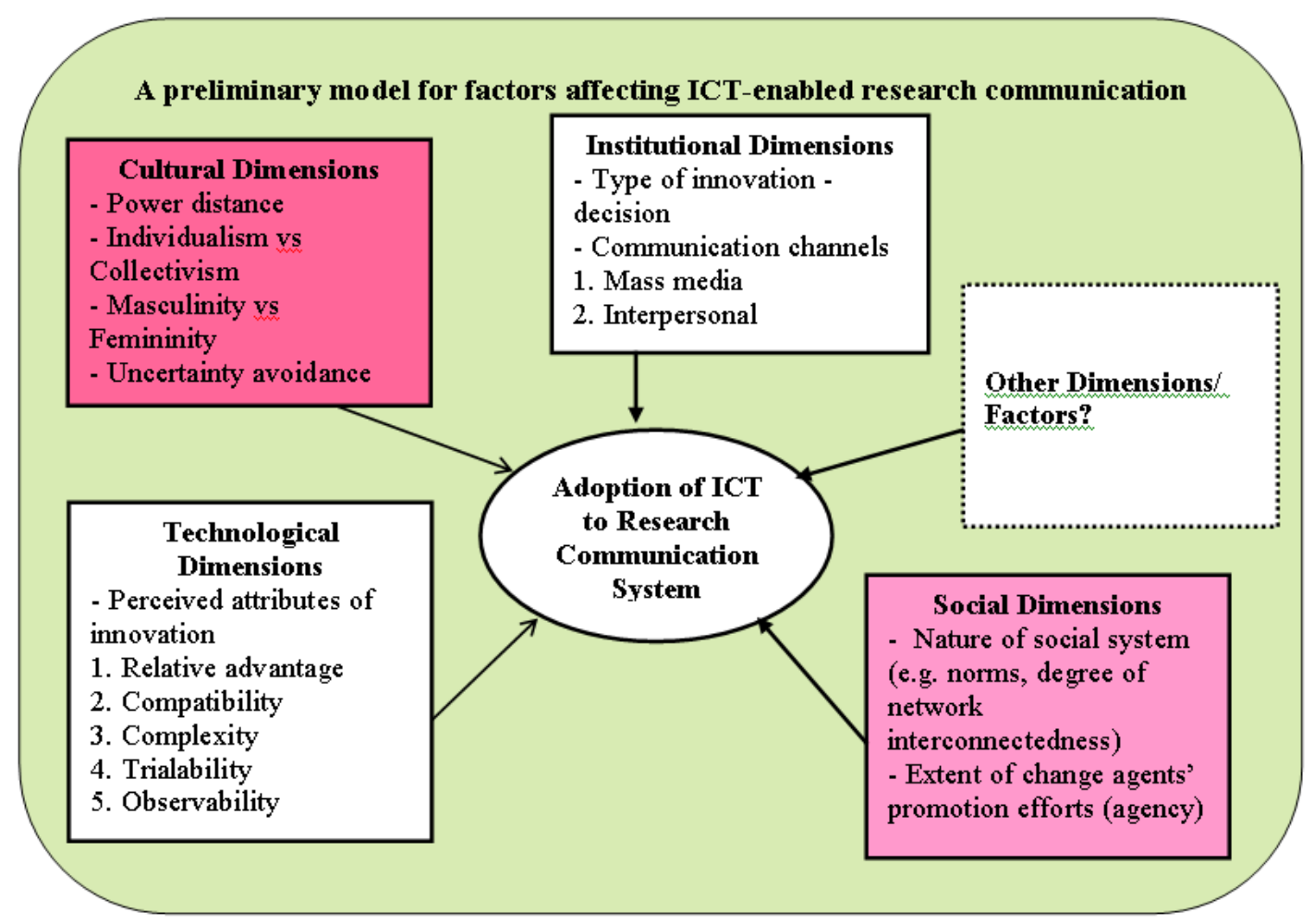

\subsection{Chapter Overview}

The previous chapter explored the emerging priority research communication needs of researchers and scholars in Kenya. The overarching research communication need was to reinforce the capacity for strategic research. One way participants felt this could be realised was through prioritising and investing in scientific and technological research communication, which they said should include improving research communication tools and infrastructure, and especially Internet connectivity. This chapter and the next two pick up the thread from there to present findings and discussion on the barriers and enablers to ICT-enabled research communication by researchers in Kenya in order answer research questions 3 and 4: 
- What are the contextual factors that affect ICT-enabled research communication by researchers in Kenya in agriculture, biology/biotechnology, environmental science and health sciences?

- How do these factors affect ICT-enabled research communication by researchers in Kenya in agriculture, biology/biotechnology, environmental science and health sciences?

This in turn paves the way for the presentation of a contextual framework for the adoption of ICT for communication of scientific research outputs in Kenya.

Three main factors affecting ICT-enabled research communication emerged from the data:

- Socio-cultural factors

- Organisational factors

- Technological factors

Other factors that also surfaced but were not reflected in the preliminary model included:

- nature of discipline/type of data

- Personal/individual institution's initiative

- Telephone wire thefts

- Lack of ICT research.

\subsection{Socio-Cultural Factors}

The findings presented in this chapter affirm the innovation diffusion and cultural theoretical perspectives (Hofstede, 2001; Rogers, 2003) guiding this study, which indicated that social and cultural environments affect the adoption of any new innovation like ICT. This was also supported by other studies (Ali \& Brooks, 2008, 2009; Ali, Brooks \& Alshawi, 2008; Bagchi, 2001; Bagchi \& Udo, 2007; Casal, 2007; Duque, et al., 2005; Galivan \& Srite, 2005; Keengwe, et al., 2009; Musa, et al., 2005; Nath \& Murphy, 2004; Weisinger \& Trauth, 2003). Borgman (2007) underscores the 
need for an understanding of the context on which to base an ICT adoption framework for research communication. She points out that,

...the development of scholarly information infrastructure has focused much on building a framework to support any kind of information without regard to its meaning ...than on building a framework to provide context for the interpretation, use, and reuse of content- an infrastructure for information (p. 149).

The researcher has opted not to separate the social and the cultural factors as presented in the theoretical framework (see figure 3.2) because the social life in the Kenyan context merges into the cultural and vice versa.

Participants gave examples of the socio-cultural system that hindered ICT-mediated research communication. These included low ICT appreciation; perceptions about ICT potentiality and use; attitude of the research community; demographic factors; lack of/need for connections/networks for ICT adoption and traditional cultural values.

\subsubsection{ICT appreciation}

The participants felt there was lack of ICT appreciation in both the internal and external institution environments. They attributed the lack of ICT appreciation to factors such as lack of understanding and fear, which are discussed in the following sub-sections.

\subsubsection{Lack of Understanding}

Participants reported that lack of understanding of the importance of ICT in information management and communication by decision-makers across the board led to lack of appreciation for ICT and its potential contribution to development. Many from the highest level of decision-makers, both nationally and institutionally, who are responsible for planning and allocating government resources for implementation of government activities and programmes, seemingly did not place much importance in ICT, with some reported as not even having email. Owing to this lack of understanding, a government official noted that the computer-mediated communication was viewed as "dangerous to state security” and its adoption and use for communication in government transactions was not allowed. A lecturer commented that the Legislature, who should champion the adoption and 
implementation of ICT, did not understand its usefulness in developmental activities and felt it would be "empowering their constituents too much."

The general lack of understanding of the potential contribution of ICT to development led to low or lack of its prioritisation when it came to budgeting and resource allocation. In fact the findings revealed that in some institutions ICT was not a budget line, so no resources were allocated, making it difficult to adopt ICT for research communication as a senior government official, researcher and library manager reported:

... as I've said here a thousand times, you will only be lucky if the people in the administration have some sense of appreciation of ICT... So you would be lucky to have a budget for ICT (GO1_Ma)

... we had a meeting where the ministers were questioning: I pay you as professors to do teaching. Now you are asking me to pay up for all these learning systems. If we have learning systems, then I have one person from Makerere University probably teaching all of East Africa. So do I need you now? (RS1_G)

A lot of us are ignorant of the value of ICTs in information management and handling...It is not really true we don't have the money ... We are talking of decisionmakers whom we are trying to tell we need wider bandwidth in order to access electronic resources and they are ignorant -they don't know what you are talking about, they don't appreciate it. So it becomes very, very difficult in a lot of institutions... We are talking of politicians themselves who do not even have an email in this 21st. century... (L1_A).

Many in academia were reported to lack computer literacy and so they were hard to convince about the merits of e-communication. A professor reported "some of the older professors were not up-to-steam with it [e-communication] and they didn't see the purpose” (RS1_G). A scientist added, “... our people [researchers] still don’t understand how we can use this technology...” (RS5_D). Hence, they were said to still insist on having printed copies on library shelves and students presenting their work in hard copy:

You come down to the university level, and again this is from my own experience, our own professors, quite a number of them, cannot use computers and you are trying to tell them that there is electronic information out there, they argue with you I still want my print copy on the shelf... (L1_A)

The professor still waits for a hardcopy ... (LR2_A).

A communications official reported a general lack of understanding about communication, which he saw as affecting the process of ICT-enabled research communication. This was supported by a lecturer who argued "there was an element 
of ignorance [by governing structures] ... in understanding the importance of communication... it is only the lack of recognising that communication is very, very key in enabling the country achieve substantial developmental steps” (LR2_A).

Some participants explained the lack of understanding of the potential contribution of ICT to the country's development on the part of politicians and senior government officials made them unable to challenge the existing monopoly of the telecommunications sector by the government. This resulted in Jambonet being the only Internet Service Provider for a long time. A lecturer cum ICT director was of the opinion that the monopoly by the state-owned Telecom did not create the necessary competitive environment which would help improve the push for business and efficiency in telecommunications and so hasten ICT adoption.

As the participants suggested, there was need, therefore, for intensive and extensive ICT awareness campaigns to sensitise both researchers and law/policy-makers about the role that ICT could play in the development process so that they could prioritise it to facilitate ICT-enabled research communication. They needed to be made aware in order to act as champions for ICT issues in parliament, government and research institutions respectively, to facilitate faster adoption. Moreover, a library manager felt there was need to invest in and introduce computer-mediated communication right from primary school to create early awareness and exposure.

The findings affirm the literature (Mutula, 2001; Ondari-Okemwa, 2002; OderoMusakali and Mutula, 2007) and align with Rogers' (2003) proposition that there was need for champions and early exposure to any innovation to be understood well enough to be adopted.

\subsubsection{Fear}

In addition to lack of understanding discussed above, participants cited fear as another reason for lack of appreciation for ICT in communication of research outputs. A senior research scientist indicated that ICT "is a technology that people are still inherently afraid of” (RS5_D). A research communications advisor added there was "general fear of the unknown" regarding ICT technology and that the "older generation people fear looking foolish and learning new things ... fear showing that they don’t know how to turn on a computer” (GO1_Ma). The fear factor resulted in a 
gap in utilising ICT for research communication. A library manager reported many public research institutions were at the initial stages of computerisation and struggling to computerise their libraries. The delay had been occasioned by fear of ICT which in the past was a "no-go-zone. People feared technology and they didn’t want to touch it," (L2_E).

The uncertainty surrounding ICT had also resulted in scientists' unwillingness to communicate-what one government official called "inelasticity of communication between people because you have gotten fears which are building as a result of inadequate training” (GO3_F). Interviewees reported that many researchers were afraid of using computer-mediated technology to communicate their research for fear of plagiarism, especially when research output was unpublished. They feared they would have no control once the work was in the public domain; yet, science is competitive, more so due to lack of funding and the fact that it takes long to come up with results. Researchers feared losing their Intellectual Property Rights (IPR) to more well-endowed competitors who might take advantage and publish the results, thereby claiming ownership. Interviewees reported instances where some "people have sent their proposals for funding and have probably found them executed elsewhere" (LR1_A) and others have had their work pirated without acknowledgement, "even if you put a rider asking people to acknowledge when they cite you, people will still not do so,” (RS6_C). This was made worse by lack of understanding of e-security and the necessary skills to safeguard their work against plagiarism:

The problem is if you are not actually very compliant with ICT; how do you actually know whether actually what you are sending is secure enough? They have no way of knowing. So they take time for them to understand. ... if you are not at that level [where you can protect your work] then you have actually an issue. I have seen it here; I have seen it in developed countries. So it is universal: the security of actually ICT communication ... (LR2_A).

Thus, inadequate ICT skills aggravated the fear of computer-mediated communication and e-publishing.

A lecturer indicated this was among the greatest barriers to ICT-enabled research communication:

... if you did a study and probably you didn't have very huge numbers [not using ICT] purely because of funds limitation it is probably because of that idea that 
somebody else can pick that idea and because you didn't have funds can go and duplicate that idea better... (LR1_A).

All in all, however, fear was reported to be diminishing nationally and many people were using ICT in Kenya as could be witnessed from the packed Internet cafes-an indication that ICT has been accepted and its usefulness appreciated.

A senior government officer indicated that the push for ICT, as evidenced by the presence of satellite dishes in many places, "will put out information whether some dictator wants it or they don't want it” (GO2_Mb). Moreover, the current government understood the importance of ICT-mediated communication and has spearheaded development of the E-government Strategy (Government of Kenya, 2004) to guide the ongoing implementation of ICT in government ministries and departments. The government has also spearheaded the development of a Science, Technology and Innovation (STI) policy (Government of Kenya, 2007) to mainstream science, technology and innovation capacities and capabilities by leveraging new and emerging ICT. In addition, two ministries, one in charge of higher education, Science and Technology and the other in charge of ICT, have been set up to address ICTmediated communication and higher education, science, technology and innovation.

Nevertheless, some researchers and librarians from both national and international institutions reported that ICT adoption at the institutional level, although picking up, has been slower than at the national level. Participants said it is only now that public institutions have accepted and are using email for communication. One research scientist reported the issue of "early adopters and, of course, the laggards" who were "reluctant to adopt because they want to check the effects on the early adopters" (RS6_C). This scientist reported there are those within the research community who "even the mobile they have refused to adopt." The laggards seemed to be "more comfortable with what I know" as indicated by one lecturer; thus, affirming Rogers' (2003) DOI theory about early and late adopters in relation to any new innovation. The tendency to resist ICT adoption for fear of risks also aligns with Hofstede's (2001) idea of Uncertainty Avoidance, where people tend to avoid the unfamiliar for fear of negative consequences. This underscores the need for massive awareness campaigns before introduction of any new technology. 
Participants attributed this fear of adopting and using ICT both nationally and institutionally to the way computers were introduced in Kenya; job security; health risks; information security; uncertainty about quality control; possibility of miscommunication and loss of control/expertise.

Participants explained the way computers were introduced in Kenya without prior awareness creation or training to demystify the technology by explaining its functionality and what people stood to gain or lose affected its adoption. Some participants commented people had no idea what a computer looked like, which resulted in threatening tales about it. A government official cited rumour-mongering that likened the size of the computer to "a big room somewhere in Chiromo", with its sole function as a big salary processor "that, if your name is not there by some date, you are sure you will not even earn your salary;” thus, scaring people.

Due to lack of prior sensitisation and training, fear of the computer continued even after the coming of PCs. A senior government officer said this resulted in "senior officers who had no clue how it [PC] works... and, fearing the challenge of younger ones, they virtually locked them up. This country is replete with stories of computers which expired because of unuse ...” (GO2_Mb). A library manager reaffirmed this:

Some 7 years ago things were quite bad in Kenya in terms of adoption of ICT. You would find ... where they got donations of computers which were locked in a store until they became obsolete before they were used. There was the fear of this technology (L2_E).

Thus, there was need to build awareness to prepare institutions for the introduction of ICT.

At the institutional level, there was fear that the efficiencies associated with ICT would affect the employment rate. A library manager, a lecturer in charge of ICT implementation in university $\mathrm{A}$ and a communications officer in research institute $\mathrm{E}$ reported that the benefits of ICT have been at the national level in terms of reducing unemployment, but that computerisation at the institutional level has indeed led to redundancy in many institutions. They gave the example of VOIP which rendered entire cadres, such as secretarial staff, jobless. They felt such scenarios instilled fear and resulted in a go slow in ICT adoption. 
Fear of loss of extra income from overtime as ICT increased work efficiency, was also suggested by an ICT officer in a public institution. Hence, people resisted the adoption of ICT. Moreover, this officer added that the transparency of ICT-mediated operations and communication caused those working in sectors open to corruption to fight ICT adoption for fear that their dubious deals would be exposed; thus, leading to loss of income.

Thus, as one lecturer explained, "most of the user resistance to the adoption of ICT is actually ... trying to avoid risk" (LR2_A). A government officer commented, "the general fear of the unknown is a reality, particularly adopting the computer ... So it is in a sense something that you might call cultural, a fear of the unknown” (GO1_Ma). As a result, "We have seen these incidences where people seem to try and avoid these new technologies [because] they are not so sure they are for their own benefit or good,” (RS3_D). Hence, job-related fears were a significant barrier to the adoption of ICT within government institutions.

Another reason for reluctance to adopt ICT reported by a library manager and a research scientist was the perception that ICT led to health-related problems such as poor sight, radiation and infertility:

... a lot of staff are still scared of ICTs- ... excuses that I will go blind, radiation... So you find that you will get about $70 \%$ of the staff on board... (L1_A)

The problem is that it can destroy my eyes when I am always staring on the computer... I also hear it makes some people infertile ... (RS6_C).

A communications officer in institute $\mathrm{F}$ indicated danger of hacking into computer systems and stealing data made scientists reluctant to use ICT for to communicate research information:

... there is a risk of people hacking in and stealing their data and coming up with papers ...I would say scientists are the best when it comes to adoption of ICT, some of them even better than IT professionals. We call them almost wizards. So they have no problem adopting and using the technology... but there is that reluctance to openly use the medium to communicate and release the information, as it were (GO3_F).

Fear of virus attacks in institutions that could not afford anti-virus protection was also reported to have discouraged adoption and use of ICT for research communication.

In addition to fears about information security, participants raised issues about quality control in computer-mediated communication, which they argued made researchers 
reluctant about adopting ICT for their work. This was worsened by the fact that, for a long time, researchers' and scholars’ promotion had been pegged on print publications in refereed journals, as reported a library manager:

Particularly the academic staff will tell you that I cannot be promoted if my publication is in electronic [format]. So it is an uphill task to convince them ... Most of them are not yet quite comfortable with it (L1_A).

The uncertainty was said to be more prevalent among older generation researchers as opposed to the younger generation. One university Professor underscored the need for a paradigm shift, where researchers' promotion would be based on research products, rather than publications, if research were to inform industry and development in general.

The data also revealed that many researchers, especially the scholars, were reluctant to communicate information over the Internet for fear of loss of control/expertise, owing to the fact that the Internet avails information to all. So, as a lecturer cum ICT manager in A put it, "whatever information you do give people have basis for challenging what you say as a professor” (LR2_A). This presented a challenge to the traditionally knowledgeable-“the experts"- in that "the professor here might suffer from the issue of mostly losing the seat as the domain expert; as, in capital letters, THE ONLY PERSON WHO KNOWS - UNCHALLENGEABLE... losing control over information I had; control which I could use in different ways ... personal gain, professional gain" (LR2_A). He further explained that "they [professors] feel threatened- it is as if you are taking something away." Consequently, "they hide under IPR' and "refuse [to have] their lecture notes ... put into the e-learning system where they can be accessed,” he added. A library manager further illustrated this by sharing her experience in university library automation project:

...the people who were supposed to move the project were not computer literate and although some of us were, maybe there was also fear of power...I don't know how to handle these things [computers]; my junior knows how to handle them...fear of loss of power can affect the speed of adoption... we still have the lethargy (L2_E).

This upholds the findings of Ondari-Okemwa (2002) on the power wielded by lecturers who feel threatened by the adoption of ICT for teaching and learning in universities.

It is not only the scholars and researchers whose authority and control was threatened by the efficiency and transparency of ICT, but also those with vested interests. For 
example, examination administrators feared no one would consult them; journal suppliers and librarians feared losing business. Accordingly, they might, therefore, create hurdles for ICT adoption, as a lecturer reported:

Even with administrators, if I am the chief examination officer and I have to put all the results on the intranet or even the web or Internet, now I seem to lose control -no one is coming to apply to me; no one is coming to beg me; no one will even bribe me... it creates uncertain future for our university suppliers and even for the librarian and for the entire group who are actually working with these physical journals, those cataloguing, etc. even by the fact that by the touch of a button I can access the whole international standard catalogue scheme and download it ... then all procurement staff and the circulation within the library have their jobs threatened ...others may try creating hurdles, user resistance... (LR2_A).

The Internet was also perceived as creating an uncertain future for the leadership/managers if "their subjects have too much information", which might empower staff to push for more accountability in budget allocation and procurement, as argued by a lecturer cum ICT manager:

... if you make management information systems records available on the network, then the budgetary allocations...; budgetary, procurement plans and strategic plans, are known to everyone, then the managers in the administrative places will now be faced by users who are demanding (LR2_A).

He added that the leadership felt safer without the exposure brought about by ICT.

It emerged from the data that the fear of losing jobs and control by those with vested interests was so great that people did not recognise the new opportunities offered by ICT, such as new jobs, job expansions, increased student enrolment, more library subscriptions for less money and broader access. It is this fear of loss of authority in a culture that views the educated as all knowledgeable, and where challenging those in authority is seen as culturally impolite, that the adoption of ICT for research communication has been slow.

However, attitudes towards ICT were gradually changing, as one lecturer reported:

You know the bad or good thing about change is that you either go with it or change will actually change you. Whereas we are faced with some resistance in some situations, generally we seem to be accepting (LR3_B).

Thus, there was hope of increased adoption and use with time.

\subsubsection{Perceptions about ICT potentiality and use}

Participants raised issues regarding researchers' perceptions about ICT use in research communication. Some of the concerns had to do with the sense that there was unclear 
perception about the functionality of ICT and its impact on researchers' creativity. Participants reported that there had been a tendency to regard ICT not as a facilitating tool but as an end in itself. The data point to instances where people thought ICT could perform functions that only humans can do, such as, for instance, creating, sorting and packaging the information to be communicated online and actually uploading it to the website:

... some of the centre directors were complaining that when you go to their websites you don't see anything more than mandates of their centre on the websites. He then told them, "if you don't provide write-ups of what you do in your centres, what do you expect to find in your websites?”

This resulted from lack of or inadequate training or sensitisation on ICT use and potentialities, because most ICT initiatives were donor funded, with the donor agenda focussing on ICT infrastructure and tools, rather than capacity building and content. This was said to be worse for those in remote locations with poor infrastructure.

The unclear perception about the functionality of ICT and ICT-mediated research communication led to lack of faith and, therefore, distrust of e-publications by institutions, which for a long time insisted on hard copy peer-reviewed publications for any promotions, as the following comment by a scientist illustrates:

And then it is like it is not so authentic [e-publication]... You know, it is like you have to convince the people that this paper is indeed authentic. ... There is no longer that train of communication that you can show. It [e-publishing] has become so simple... So there is that lack of faith (RS1_G).

This discouraged many, especially older researchers, from ICT-mediated research communication.

Poor ICT perception withstanding, participants reported in institutions where ICT functionality had been clearly perceived they were populating their websites with useful information like detailed CVs and research publications. These provided information on research in Kenya and served to create collaboration platforms. It was reported that some centre directors and other senior researchers even had their own websites and the research outputs from their departments were already online.

However, there remained the perception that ICT-mediated communication could lead to stifling of researchers' creativity. Some participants felt there was the danger of too 
much focus on ICT tools at the expense of engaging in productive research. A scientist reported,

But I think we can also get obsessed. There is also a level of obsession with email that you have to be on email... There will always be obsession with the communication tool which would be negative aspect with communication... (RS3_F).

Another one argued it was easy for scientists to lose their creative edge in that Internet “might hamper my creativity and thinking space” (RS4_E), needed to develop methodologies and originate and communicate useful research outputs "simply because I have a computer on all the time.” Another scientist added that researchers risked stifling of their creativity by "believing everything they get from there [Internet]. They should believe less and do more analysis” (RS1_G). Thus, researchers did not seem to be analysing or synthesising whatever information they got from the Internet, in order to meaningfully integrate it into their work.

The findings showed that obsession with the Internet was not limited to the scientific community, but also seemed to be affecting students in schools and the universities. A scholar reported “... the younger generation ... is so much hooked on email, and other people's creativity might stop them from being originators. But then they begin to copy because it is so easy. ... kids will go to the Internet to cut and paste and write that essay and give it to you” (LR4_B). Thus, the young were not learning to compose and write.

This accords with literature that discusses the "Internet addiction disorder" where the young seem to be the greatest culprits (Young, 1998; Arunyanak, 2004; Buckingham, Whiteman, Willet \& Burn, 2007). Bittman (2008 ) in his New York Times March 2008 article, "I Need a Virtual Break. No, Really," argues that there is need for a "'secular Sabbath'... a day a week where I would be free of screens, bells and beeps,” which he terms "the latest in American problems, Internet addiction disorder" (n.p). He argues for "the need to turn off, to take a stab at reconnecting to things real rather than virtual...” Bittman reports that by taking time off the computer, "I felt connected to myself rather than my computer. I had time to think, and distance from normal demands.” He further quotes David Levy, professor at the University of Washington Information School, who coined the term "information environmentalism," on the need for time off: "What's going on now is insane. Living a good life requires a kind of balance, a bit of quiet. There are questions about the limits of the brain and the 
body ... Who would say you don't need time to think, to reflect, to be successful and productive?” (Bittman, 2008, n.p). He also cites a spiritual consultant, Anne Dilenschneider, who holds that "Even many corporate leaders now believe you need time to hear the voice of the new inside.”

Thus, the concerns about ICT addiction usurping valuable time that scientists need to focus on research to produce results are real. There is need for early awareness on this and clear strategies and mechanisms to avoid stifling of creativity among researchers.

\subsubsection{Attitude of the research community}

Rogers (2003) defines attitude as "a relatively enduring organization of an individual's beliefs about an object that predisposes his or her actions” (p. 174). The findings in this study reported that attitude, said to have developed as a result of social inertia, was a significant barrier to the adoption of ICT for research communication. As a lecturer explained, “... society tends to propagate what it is used to. So many of us actually have dealt without some of these gadgets like PCs and what have you and without using certain softwares and so there is tendency to continue doing what you have always done” (LR3_B).

The slow adoption of ICT for research communication in Kenya seemed to indicate what one research scientist called "entrenched mindsets and attitudes” (RS6_C) where researchers and scholars saw the need for ICT but inertia made them slow to adapt. The slowness to change was said not to appertain only to ICT but also to the education system where an old curriculum was still used despite its out-datedness. Although the curriculum is outside the scope of this study, it could be an area for further research, where one could investigate how the current curriculum in a country affects the adoption of ICT for research communication.

A research communication advisor attributed the researchers' non-adoption of ICT to their being "steeped into a social and cultural belief which impinges against change, against uncertainties, against innovations...” (GO1_Ma). Many institutions were reported to have started having a web presence in 2000, but even then most of the websites were said to be inaccessible, as excerpts from a government official and a research scientist show: 
... scholarly community has been very slow particularly in Africa to respond to the era of ICT and the possibilities provided by the Internet. I'm not so sure that anything has changed for the last 4 years in publishing by scholars from Kenya. ... The best university A has done is ... establish a web site which for many years was totally inaccessible... the first signs ... was around 2000? (GO1_Ma)

... we are still moving very slowly because people have not really understood and appreciated how to use ICT. We are still way back there (RS5_D).

Participants argued that social inertia had resulted in a lack of proactivity in anticipating change among African/ Kenyan scholars and researchers. A participant observed that, unlike the Western world where people hasten to upgrade skills when faced with new things, this was not the case in Kenya. A research communications official noted there were no programmes in the research institutions to promote/spearhead ICT adoption except for self-driven initiatives.

A scientist commented that the slow adoption of ICT by research institutions was due to the fact that the majority of scholars and researchers were yet to see the need for ICT or capitalise on it; the technology was new and unfamiliar to them:

Some people even are afraid on how to use the computer and workstation. Even researchers some of them now are learning how to use the computer before using the facilities that information communication technology can give them. So progress is slow because the area of ICT is still very new even for an institute like this... We are still very slow. These people have not appreciated how they can use these facilities and that is where the problem is (RS5_D).

Where institutions had internet access, "the incidence of use towards research is actually very low,” as a lecturer (LR2_A) indicated.

The non-use of ICT by the research community is affirmed by studies on ICT use in research institutions in Kenya, especially the universities (Mutula, 2001; OndariOkemwa, 2002; Odero-Musakali and Mutula, 2007). Mutula (2001) argues:

Kenya has fairly well developed IT infrastructure to support the IT initiatives and networking necessary for vibrant academic research development. However the situation at the public universities in Kenya does not reflect this potential... The universities have not woken up to the reality of the new technologies, and have not exploited them effectively in their academic programmes to encourage wide use of the new technology (p. 163).

He attributes the situation to lack of initiative by the research institutions: "The infrastructure may not be the best, but what efforts have so far been made by the universities to use private communication networks that are widespread in Kenya?” (p. 163). He compares the non-proactivity of the Kenyan universities to the 
proactivity of universities in Southern Africa such as, the University of Zambia, University of Botswana and several universities in South Africa who are ISPs for their own countries. On the other hand, if we consider diffusion theories, such non-use could be attributed to Hofstede’s (2001) view on Uncertainty Avoidance when faced with a new innovation and Rogers' (2003) discussion on the need for adequate exposure to any new technology to maximise adoption; thus, calling for champions to spearhead the adoption in Kenya.

A government official considered that "general laziness, like is endemic in every civil service" where "an officer will buy a computer which will sit there and it would be covered with sheets for everybody to know the officer is computer literate but he doesn't touch it" had affected the adoption and use of computer-mediated technology for research communication. He described the researcher in the public institution as "not [having] particularly adopted the use of the machine and he doesn't want to bother himself” (GO2_Mb). A senior researcher concurred with the idea of laziness and described researchers as being "downright lazy... They are lazy and arrogant” with “a disappointing attitude and backward misconception” (RS5_D). He reported that senior scientists who lacked computer skills did not want to admit they were ICT illiterate. These two participants explained that the attitude was as a result of the prevailing culture in Kenya, where the computer in public offices is treated as a symbol status rather than a tool for work. Such an attitude was said to have hampered the adoption and use of computer-mediated technology. This is an issue appertaining to high power distance where seniors did not wish to admit ignorance or that they could learn from the juniors in an organisation (Hofstede, 2001). This suggests the need for a cultural-attitudinal change for faster ICT adoption, as one participant underscored:

... we need some institutional changes. The thing is people like doing things the way they are used to. But now we want to do things in the unusual... If I tried to tell them to embrace other approaches it will be very hard... (RS6_C).

Despite the attitudinal problems discussed above that have slowed the adoption of ICT for research communication in the past, a government official reported "there is a light at the end of the tunnel” in that changes in attitude were beginning to be evident as ICT permeates and becomes more accepted; thus, demystifying the computer technology. He explained that, due to the government policy that waived tax on 
computers, researchers were now beginning to buy their own and bring them to offices where not provided.

\subsubsection{Demographic factors}

Apart from researchers' attitude, participants reported demographic factors such as age, gender and literacy level as affecting ICT-mediated research communication. The next sub-sections will address these factors.

\subsubsection{Age/level of qualification/exposure}

"There is a certain generation that has to go in some institutions for them to really adopt ICT” (Library Manager, research institute E)

As the above excerpt denotes, age was reported to be a major barrier to ICT adoption for research communication by public research institutions in Kenya. Many in decision-making positions that impact on issues of ICT adoption were said to be old and lacking an understanding of the importance of ICT. Consequently, many of them were reported as not seeing the need for integrating ICT into work operations despite having many qualified and competent personnel, some of whom had studied abroad in the developed world, to spearhead implementation. A library manager gave the example of the difficulties faced in computerising libraries in public institutions where old computer illiterate library managers did not want to let the young computer literate librarians spearhead to the process. She contrasted the slow ICT adoption in public institutions with the fast rate of adoption in private universities like Strathmore and Daystar, which, though new, were headed by young and dynamic personnel who were reported to be already ICT compliant and agile users of ICT. She concluded:

... So you can see the relationship between age and the adoption of ICT. The younger, the faster the rate of adoption. Today, if I write an email to $\mathrm{Y}$ [former public University Librarian], I never get a response but if I write it to the Strathmore librarian, I will get it immediately (L2_E).

Thus, the age of the institutional leadership was a critical factor in ICT adoption and the findings showed that those institutions headed by young and exposed leaders were making great advances in implementation of ICT-enabled research communication. Conversely, older leaders tended to be non-adopters of ICT, for they lacked training and exposure. One senior government officer and professor referred to them as "Old deadwood...old fashioned fellows who were not well trained in IT... allergic to the computer” (GO2_Mb). A library manager added that “...the age comes in where people don't want [ICT] because they are old” (L1_A). A research communications 
advisor reported some old professors did not have the will to learn even how to send an email and "are the ones most likely to ask a person to check an email for them" (GO1_Ma).

The few older ones who wanted to learn about ICT were discouraged by the fact that it took time to learn and their age and busy schedules did not allow them to pick things up as fast as the young. So, late exposure and social-cultural inertia held them back, as a participant indicated:

... first of all they didn't learn about it. Secondly, it takes a little bit longer to teach an old person a new trick than a young person unless both of them are really being forced by the circumstances to use it. If I send an email some of these young guys check their emails almost every day but if I send an email to somebody who is almost 60 some of them check their emails maybe once in a week or sometimes once in two weeks because I mean there is what you grew up with and there is what you are now being asked to learn ... (LR3_B).

It could be said the young are more adoptive than the old. One researcher commented that "the old scientist has for a long time has been used to doing things using a very crude way of handling his research. ... The young ones want to do things quickly and they know where they can do that. It is the new technology which provides that" (RS3_D). Thus, the old scientists were conservative, whereas the young wanted quick fixes that the new ICT made possible. As a lecturer explained, "Whether it is the use of an LCD projector in a class or to use Internet for research, the younger ones will be in the fore front of adopting ICTs,” (LR2_A).

As another scientist of medium age reported, the old, owing to their social status, might also not want to display their ignorance or inability to handle ICT. He explained that the young, who, were exposed early to ICT, were more knowledgeable and confident about its use:

... they [old researchers] will find like I'm supposed to be like a very educated or somebody who knows a lot yet the new technology is putting up a big challenge ... they don't want to be seen they are not compatible, they are not matching the new technology... The young guys are very good at putting up with the new technology, better than the old ones ... They just tell us things that they are able to do using the new technology in a quite convincing manner (RS2_D).

A lecturer explained that owing to early exposure and skill, easy demeanour and flexibility, "the young Turks get more excited by the technology and they pick it up rather faster than the old Turks,” (LR3_B). They were reported to have embraced ICT more than the conservative old researchers - "the story of the old dog and new tricks 
becomes a problem,” (GO3_F). A senior scientist reported that, although the young are not taught how to use ICT, "they have a [knack] of finding how to go round it" (RS3_D) and use it a lot - "they are literally on the web” (LR2_A), unlike the old guard who did not use it as much.

A young ICT lecturer commented that the age factor tended to cause conflict between young adopters and old non-adopters in that "you will find that the students and young lecturers will require direction from this professor but then the professor has decided he wants to stay back in the old days” (LR2_A). There had been instances of some senior non-ICT compliant scientists looking down upon the young and not wanting to work with them. A participant reported about a scientist in research institute D who would insist, "I want the old guys who have lived with the institute for many years ... people who are not less than research officers... senior research scientists and above, that top echelon kind of level” (RS3_D).

Traditionally, there was high power distance between the senior and young scientists with the old mentoring the young in research. However, this was now proving difficult, in terms of computer-mediated communication, such as presentations skills, word processing and internet search skills, as one senior lecturer indicated:

Essentially [the young] learn from these old ones... But the challenge where the old have not done it properly is I think to make sure that these young people use... ICT infrastructure like ... PowerPoint projector when doing presentations, ensuring they process their work using the computer and also use the Internet for reference materials. It is a bit tied to what you as a person know. You know you cannot give what you don't know, what you don't have (LR3_B).

Nevertheless, the force of change was obliging older scientists to adopt ICT. For the professors and lecturers, the demand for ICT-enabled communication by the students, in addition to growing appreciation of the potentialities of ICT, was forcing them to adopt the technology to save their jobs, as indicated by two lecturers from university A and B. As another lecturer observed, students are no longer interested in traditional communication and,

....are asking [for] quality projection. So it is either you are with them or without them. So you find actually, although a number of us will not be moving with that speed, people have no alternative [other] than to move with the ICT... they prefer a person who goes with an LCD and a computer and projects and at the end of the day can tell them this kind of information you can get from this website... (LR2_A).

Thus, the force of change is gradually enforcing ICT adoption. 
A. Limo (2008) talks of the inevitability of e-learning as the tool for future even in Kenya: “The new method of learning, popularly known as e-learning (electronic learning), is the tool for training for the future. The 21st century school will stock “talking books" or e-books" (n.p). He hastens to add "Computers will make a difference in education for those who can afford the technology and are willing to use it. The mark of success will not be the presence of the computer but rather its use."

With the inevitability of ICT-mediated research communication, a senior scientist and head of a department reported changes were occurring and the reverse was beginning to happen in Kenya with the young mentoring the old:

I call the young ICT staff to teach me what is in the market now... my director does the same (RS5_D).

The young helped the old who were willing to manage information and, in the process, communicated with them via email and helped mentor their elders, as an ICT lecturer reported:

... some times what happens is that this old person will actually give this young person the task of now going to use the computer to do $\mathrm{x}, \mathrm{y}$ and $\mathrm{z} . .$. and in the process may be they, the old guard, learn a few things because may be the young one will send the old one an email telling him what he has done.... So these guys [young] have now actually been able to do this work for the old... (LR1_A).

A director of ICT and a scientist argued that those still being mentored by old professors who cannot access Internet had to gradually update their mentors. They said this was important because African culture demands respect for the old and so young researchers cannot challenge an old Professor about what he does not know from the Internet:

... in Africa we have a culture of respect [for] the old people; so, if there is an old professor who is not going to check some of these Internet or this and that course, I wouldn't go challenging him telling him ... No we don't. We respect them. So there is no fight about that but the point is that I should go there [Internet] and find out and then I can bring it to him and we can discuss... (LR3_B)

In fact we get lots more updates from younger researchers about process. I will get more information coming into my email from younger researchers simply because they have access and time to browse the Internet for opportunities... (RS3_F).

Thus, the young ICT literate researchers were informing the elderly about research issues, since they had the skill and time to be on the Internet. 
This kind of relationship between old and young researchers reverses Hofstede's (2001) idea of the social consequences of high power distance due to age and level of education/authority in countries with high power distance traditions like the case in Kenya. A lecturer reported that ICT has made it possible for the young to engage in peer mentoring in areas that the seniors could not deliver:

... the challenge more comes from their peers rather than the old generation because you know you will see what your friend who is also doing research in another area is using, like what software s/he is using to do $\mathrm{x}, \mathrm{y}, \mathrm{z}$ and then you try and get that software... even if you have not been told by your supervisor to do that. Then when you show your superior as long as it is good, I'm sure s/he will appreciate it. So it is more of peer challenge for the young rather than from the old to the young (LR3_B).

Thus, ICT is minimising potential roadblocks detrimental to young scientists, as a communications officer added, "because if you are my mentor and in your department I try to [do] whatever you refuse, there are other people working in the same field even if it is not here. [I] can network, do my drafts, email to somebody to advise, do corrections and then do further readings, ... analyse this up to this point and still find [my] way” (GO3_F). Therefore, increased ICT adoption is helping to reduce the power distance owing to age and level of qualification/authority and to increase efficiencies in research communication.

The future of ICT-enabled communication in teaching and research in Kenya is quite promising because, as a senior lecturer explained, "the young people we are producing... are better ICT compliant than us and therefore they are going to make better teachers than we are and they can actually go manoeuvre, search and all that kind of stuff... So we expect things to change in this part of the world with time" (LR1_A). This scientist predicted the possibility of paperless offices in five years (2012).

The rapid changes in ICT adoption happening in Kenya due to the skill and efforts of the young researchers and scholars, and the waning influence of the old generation researchers and leaders, validate the opening statement of this section, requiring a generation to pass before ICT could be fully adopted and integrated in public institutions. Another research scientist confirmed the same:

So I can see that approach [ICT] coming to be very useful to the country because the old ones are phasing out and the new ones are taking on the old ones' positions (RS2_D). 
The high power distance that has affected the adoption of ICT in research communication in Kenya, owing to age difference, partly aligns with Hofstede's (2001) idea of the old and the learned being all knowledgeable and mentoring the young. However, it also differs by depicting the unique Kenyan socio-cultural scenario in adopting and utilising ICT for research communication. Evidence from the data showed the young are now beginning to mentor the old researchers in issues pertaining to ICT-mediated research communication, which the old researchers are not proficient in. Limo (2008) stresses the need for attitudinal change management for ICT adoption to succeed: "The first hurdle will have to do with change management. The attitude, 'I am too old to learn computers', or 'I have been achieving good results without computers' must be fought and won” (n.p). So, there is need for those introducing technology not only to address issues of hardware and software but also ““"peopleware”, a critical component for success that is often ignored,” because if you do not do so "they will tell you technology is not for them, they were 'born before computers' (some call themselves the BBC generation)”(Limo, 2008, n.p). He explains part of this change should also include dealing with the "myth of the 'computer teacher'. In the e-learning environment, every teacher is a computer teacher and so they must learn to operate a computer on their own” (Limo, 2008, n.p).

\subsubsection{Gender}

As well as age/level of qualification/ and amount of ICT exposure, participants gave varying views about the impact of gender in ICT adoption in research institutions. Nationally, a government official indicated there was a "huge connection between gender access to education and information and gender access to computer technology,” (GO2_Mb). A lecturer explained that the traditional settings favoured the male over the girl-child and added there has been unfair treatment, with "almost a deliberate encouragement of the boy child to study, to be brave, to take up new ideas..." and that "resources may not be made readily available to the girl-child..." (LR2_A). He further said that girls in some communities where there were inadequate funds were married off, whereas boys were sent to school, which he said could be seen from the male domination even in universities, even though females formed the majority (52\%) of the total population. He pointed out that the level of transition to postgraduate courses was also higher for males than females. A woman scientist further exemplified the gender divide in ICT-mediated scientific 
communication when she reported that science, too, was a male domain; so, there were fewer females in STI than males:

As for gender in ICT adoption for scientific communication, I can’t say much because there is no data but I know that over $60-70 \%$ of $C$ researchers are men... If you come to people doing scientific research $75 \%$ of them are men. You know sciences were hard subjects- biology, Chemistry ... and were initially done by men. Women don't like the sciences (RS6_C).

As a result of the disparities in education and the fact computer-mediated communication requires a high level of literacy, a senior government official and an ICT lecturer maintained that more men than women were exposed to computer technology, and so it could be argued that more men were using ICT:

I can see now you are educated and so you know how to use the computer. What about the sister who never went to school or became pregnant when she was in standard 6 and got lost in the areas of Makueni or Maua [remote rural areas] where I come from? ... So, the very factors that affect gender in our socio-cultural milieu [are] the same factor[s] that [are] affecting gender in the use of ICT... (GO2_Mb)

So in Kenya in total I would say this: with respect to adoption of ICTs, looking at this [education] background it is a fact, therefore, that you will find that there are more males who... have taken up or have adopted ICTs than females ... (LR2_A).

The above comments echo findings from studies on gender, knowledge societies and computer use in Africa and other developing countries (Commonwealth of Learning, 2000; Gadio, 2001; Hafkin \& Taggart, 2001; Stepulevage, 2001; Haddad \& Draxler, 2002; Huyer \& Carr, 2002; Derbyshire, 2003; Huyer \& Mitter, 2003; UNESCO, 2005; Huyer \& Hafkin, 2007; Huyer \& Westholm, 2007; Limo, 2008). These studies support the argument that gender disparities in education have affected ICT adoption for education and call for further study. Limo (2008) affirms initial gender imbalances in Kenya that have affected women's participation in education, science and limited the breadth of ICT adoption by women in general in Kenya: "In those days male chauvinism was common in all spheres of life and not many female students believed they too could be scientists of substance" (n.p). Derbyshire (2003) argues "technologies introduced into environments characterized by inequality tend to reinforce and even exacerbate it” (p. 43), an idea elaborated on earlier by Haddad (2002) who argued if one was headed in the wrong direction, then technology would get you there first. UNESCO (2005) argues that "the universalist ambition of knowledge societies seems to come up against the reality of the aggravation of gender inequalities” (p. 167). Huyer and Hafkin (2007) hold that “women are at risk of becoming increasingly marginalized in the knowledge society, where much more 
than access to and use of information technology is at stake. In the knowledge society they need access not only to new technologies but also to education, entrepreneurship and employment opportunities as well as the ability to participate fully in knowledgebased activities” (p.12). They call for specific actions and interventions, arguing that “given the gendered barriers, challenges and roles that determine [women's] ability to participate on an equal basis with men, the gender divide in knowledge societies cannot be expected to improve automatically with economic growth” (p. 12).

The study findings also support Hofstede (2001) on masculinity and femininity. The strong masculinity in Kenya socialised women into believing that tough careers like science were a man's domain and the computer was a "man's toy." This sociocultural inclination left women to pursue the soft options of child-minding that contributed to the quality of life for the family but left the tough and time-consuming science education and technology issues to the men; hence, the low adoption of the ICT by women in general.

Nevertheless, most participants felt that, where cultural socialisation, exposure, infrastructure and environment were the same for both sexes, the ICT uptake was the same for girls as two senior scientists, one male and the other female, observed:

But those girls that have taken it up they are just as good as anybody else ... the problem is the inertia at the beginning to pick it. That is where they need special encouragement because initially it is like this is another toy for men (RS1_G)

But when it comes to those ladies who have made it, the way scientific knowledge is passed through ICT is the same; there is no difference (RS6_C).

One male scientist even claimed that the few women in scientific research were doing better in ICT than the males, because women had to work harder in a male dominated institution and discipline to prove themselves:

Some of the people doing major studies in this compound are actually ladies. ...When it comes to using ICT, some ladies are even better than boys... Don't forget actually there is a challenge being in a male-dominated institution. You've got to prove yourself and I guess that is one way of proving yourself (laughs) (LR1_A).

Moreover, a senior lecturer and a scientist (male) reported that young women researchers were more versatile in ICT use than older women and some male scientists, with some having own computers at home: 
I'm seeing the younger women are more versatile with computers than the older ones ...we have a young women generation... who ... are quite advanced in ICT skills and their adoption and uptake of ICT is just like men (GO1_Ma)

Everybody is equally geared to knowing what to do with this new technology. The other day I was unable to do a small operation here and the lady who recently came from Egerton University ... showed me what to be done ... she told me she has a computer at home. Me I don't have [computer] ... (RS2_D).

However, there were two noteworthy exceptions to the claim that women professionals in the research seemed to be as proficient in ICT use as males. A woman scientist explained that limited access tended to restrict women scientists' Internet use. She said that, if the only access options were public places like Internet cafes, then social circumstances such as family responsibility might not allow them time to frequent them as men would:

The only thing I can just say is that because men are freer they are more in the cyber cafes than women... Do you know when men are drinking somewhere they have more time to go and read their emails and Internet more than you? So they have more time to visit the Internet than women. But for women who have made it accessibility and ICT use is the same (RS6_C).

This exception tallies with findings from earlier studies commissioned by the Commonwealth of Learning which found access to computers for distance learning was a problem for women not only in Africa but also in Europe and North America, where "men are more likely to have access to a computer at work and more likely to have priority use of a home computer” (Derbyshire 2003, p. 39). Thus, the gender divide also exists in countries with high infostates Huyer, Hafkin, Ertl, \& Dryburgh, 2005). Internet access for women depended on the available access options (Derbyshire, 2003) . In Africa, studies found that a study centre or public access facilities tended to be the only access options. Huyer and Hafkin (2007) reported that “... while comprehensive sex-disaggregated ICT data did not exist in a large number of countries, available data on women's access to and use of ICTs indicated that their overall participation in the information society, particularly in the poor countries of the world, lagged behind that of men” (p. 19). They also found there was a gap in ICT use even among the educated women: “...while the ICT gender divide tends to narrow at higher levels of education, a gap still remains” (p. 19).

Another exception was revealed by a Computer Science lecturer who argued that ICT adoption still remained low among educated women who had grown up in cultural 
backgrounds that still socialised girls as being inferior to boys. He said girls who grew up feeling inferior shied away from ICT even at university, unless they had a very strong personality. He explained there were fewer girls in BSc/MSc computer classes and, although expected to have the same ICT uptake as their male counterparts, such girls "will always take a back seat seemingly when the boys are around and not in front of the class except when the girl has a very strong personality" (LR2_A). He said girls did not want to lead groups in ICT discussions but "headstrong boys" do, which he attributed to the culture that stresses women should respect men around them:

You will see it even if you put them in a group, you'll always find that the girl is only a group leader as a compromise. Otherwise the head strong boys will have already taken the leadership roles because we have brought her up knowing that she should respect the men around her (LR2_A).

Further, he reported there were more men in ICT professions than women and added, at the university, more male lecturers had PCs/laptops and pushed for Internet connectivity than women, although their salaries were the same. He said this could be attributed to the cultural ingraining that made women shy from ICT and any other technology, including buying own cars - he argued "women are still content to take lifts here and there and being dropped by their husbands before they actually start driving themselves” (LR2_A). Hence, it could be argued the males are more adoptive in such cases. A senior scientist supported his argument: “... women particularly call it [ICT] the man's toy. The men have found it a more interesting toy” (RS1_G) and added that boys liked technology and electronic toys while girls preferred dolls.

Therefore, technology adoption had to do a lot with enculturation. So, as a senior scientist reported, the rate of ICT adoption among even educated and exposed women “depends on the background of somebody,” (RS4_E). Female university lecturers' low ICT adoption differed from female scientists', who were said to be more adoptive to technology as reported above. As this falls outside this study, it could be an area for further research.

The exceptions discussed above align with Derbyshire's (2003) findings in her study of gender issues in the use of computers in education in Africa. She found that boys tended to dominate access in schools where large numbers of pupils had to share computers, and that the computer has been viewed as a male domain: “women's and girls' lower confidence derives from the way in which computing has come to be 
defined as a male "technical” skill. Some women and girls perceive themselves - and men and boys can perceive them similarly - as outside the technology culture and consequently less competent” (p. 39). Girls’ position in computer-related learning has also been described by Elkjaer as being "guests" in a sphere where boys are "hosts" (Stepulevage, 2001; Derbyshire, 2003). The same notion is raised by Volman and van Eck (2002) who noted that "girls are far more likely to take on the role of the uninitiated even when their knowledge and skills equals that of the boys”(Derbyshire, 2003, p. 9). The exception of girls with "strong personality" is supported by a quote in Derbyshire (2003) from the Great Special Issue, in which a a teacher comments on a computer room dominated by boys: "Well, there are one or two girls, but they're the powerful type” (p. 10). The literature also confirmed that there were fewer women in STI and engineering than men (Derbyshire 2003; Huyer, et al. 2005; Huyer \& Hafkin 2007; Huyer \& Westholm 2007). Such a gender imbalance was also found to exist in Uganda, Ghana, Senegal, Mauritania and Botswana (Gadio, 2001; Ratsatsi, 2002; Derbyshire, 2003).

In cognisance of the gender imbalance resulting from the socio-cultural environment in Kenya, some organisations, especially the international research institutions, have taken affirmative action and come up with policies to counter the imbalance.

Overall, it could be concluded that, with increased education for all and greater ICT awareness, gender was unlikely to be a major issue in ICT adoption for research communication in Kenya. Two researchers and a communications official (all males) reported that ICT-mediated research communication was more related to individual aptitude than gender:

I haven't seen so many problems, at least here, in terms of say male and female because ideally what matters is who is doing the research... (LR3_B)

... there has not been any gender difference in the development or pursuit of ICT, not at all ... It is rather more of ability than an issue of whether you are a man or woman (RS5_D)

I think it is purely personal initiative. In fact most of our female scientists are doing very well. I think when it comes to ICT gender is not an issue (GO3_F).

Thus, adoption of ICT for research communication was based more on personal initiative than gender. 
Limo affirms the change which he says now threatens the male gender: "The demeaning attitude is all gone; the female student now seems to have gained the lost ground in the sciences and concern is shifting to her male counterpart,” (Limo ,2008, n.p). He further argues that ICT adoption could rid science of the gender imbalance: "If technology is used in the classroom, everyone will be a fast learner, regardless of gender” (Limo, 2008).

It could be argued this reversal of the initial trend and thought disaffirms Hofstede's (2001) views on masculinity and femininity differences in ICT adoption and gives credence to critics who argued that Hofstede's view on national culture as a uniform and unchanging entity was weak, narrow, simplistic and misleading (Ali \& Brooks, 2009; McCoy, 2003; McSweeney, 2002; Myers \& Tan, 2002; Redding, 1994; Smith, 1998; Sondergaard, 1994; Tayeb, 1994; Winch, et al. 1997; Yeh \& Lawrence, 1995). This points to the need for dynamic and context specific models to guide IS studies on diffusion of innovations; thus, giving robustness to the model resulting from this contextual study on factors affecting the adoption of ICT for research communication by researchers in research institutions in Kenya. The study employed a reflexive approach in order to also capture issues outside the limits of the DOI and Hofstede's perspectives.

\subsubsection{Poverty and low literacy levels}

As well as gender issues, the findings revealed that poverty and lack of/low literacy skills were inhibiting ICT adoption and use at the grassroots level in rural Kenya, where the target group for most scientific research, the majority of whom were poor and illiterate or semi-literate, resided. A lecturer cum ICT manager explained that $50 \%$ of the Kenyan population was living below the poverty line and argued that, whereas people might be open-minded about adopting ICT, the low per capita income meant that the majority of Kenyans could only afford "the simplest of the technology- the mobile phone” (LR2_A). This view was supported by a library manager and a senior scientist who argued that ICT affordability was an issue, especially since computer and Internet availability was largely only at Internet café outlets:

... greater part of Kenya is still poor and so adopting this technology is not easy (L1_A) 
... we also work with youth when we are documenting research work ... but whether he will access Internet will depend on whether it is free because depending on his livelihood, how much he is earning, he might not feel at liberty to spend what he is earning to sit in an Internet café. ... I'm looking at Internet as an Internet café outlet... I think the easiest way of communication will be telephone to a mobile phone (RS3_F).

A researcher explained that, owing to poverty, those in the rural areas might not know even what a computer was or what it could do, nor did they have access to a telephony system:

First and foremost, being one of the poorest countries in this part of the world I would not be surprised that if you go to the village people do not know what a computer is because they have not seen them. They have not even telephony system (LR2_A).

In addition to poverty, the target beneficiaries for research outputs suffered from low literacy levels, as some participants reported:

... in some of these zones [rural villages] there are computers; what they can do we have not explored them well and that is also based on the literacy level in this country (LR2_A)

But some of our farmers and partners are not able to use ICT because most of them are illiterate (RS6_C).

Computer-mediated communication requires high levels of literacy. This meant that this kind of communication was inaccessible to those who were supposed to utilise scientific outputs for greater impacts in socio-economic development.

Equally important is the language of computer-mediated communication (see 5.4), which tends to be English or the language of those who have developed the tools. The low literacy rate in Kenya meant few could make use of the computer-mediated ICT, which suggested there was no demand for computer-mediated communication and, so, researchers might not feel compelled to produce electronic content. Thus, illiteracy and language are key factors affecting ICT-mediated research communication in Kenya.

The findings about low levels of literacy and poverty affecting adoption of ICT confirm the literature on ICT adoption across Africa (Adams, 1997; Mbarika, Jensen \& Meso, 2002; UNESCO 2002; Mwesige, 2003; Kaye \& Little, 2004; Kebede, 2004; Oyelaran-Oyeyinka \& Lal, 2005; Gebremichael \& Jackson, 2006; Bagchi \& Udo, 2007; Casal, 2007). Casal (2007) argues that "literacy is the key element" in diffusion of ICT and added that "newly acquired "informational capabilities" can act as an agent for change for individuals and communities enhancing their abilities to engage 
with the formal institutions in the economic, political, social and cultural spheres of the life” (p. 5). This spells the need for education and training for increased ICT adoption.

\subsubsection{Lack of/need for connections/networks for ICT adoption}

In addition to demographic factors discussed in the preceding sub-section, the data revealed that lack of communication networks was a barrier to ICT-mediated research communication in Kenya (see 5.3.1). A senior scientist felt social networks would enable researchers to work together to brainstorm and iron out research information before dissemination:

Then I also need a network with people with whom I can share the information and engage in dialogue, intellectual dialogue because some of information may not be synthesised. There is information which even in my own field I may not be fully versed; so, I need others to have an equal, if not nearer equal access as well. So that network is something that you need. You need access and you need a network of people with whom you can communicate (RS4_E).

A lecturer reported that current cooperation between local universities was based on student training, rather than sharing research information, due to undue competition among universities:

Incidentally I've not seen that much inter-university collaboration. But something that I'm seeing people thinking about is for example because of my laboratory you find like Moi University probably want to utilise the facilities to come and train their students here; same with B and KU. It is based on students' training. It is not that well developed. Actually don't forget that initially many of us within the universities are competitors (LR1_A).

There was need for networks to facilitate information sharing and intellectual dialogue to help synthesise research information into forms that could make meaning when communicated online.

A senior scientist from an international institute regarded networks or consortia models as powerful platforms for ICT-mediated research communication, providing a bridge between the researchers and the target audience that would facilitate ICT diffusion to aid the communication process:

... We need to form these types of groups [consortia] with sufficient expertise to communicate so that farmers are organised in particular groups or there are cooperatives, unions, call them anything. The point is to have a provider, so to speak or call them an interpreter that communicates with scientists or with institutions of science ... so that they are able to pick up messages, interpret those messages and use those messages across... (RS1_G). 
Library managers from both national and international institutions - A, C, D, F and G - gave examples of how professional and other related networks and consortia were helping ICT-mediated research communication by providing mechanisms for developing research outputs and communicating the same. There were efforts by international agricultural organisations like the CGIAR networks to facilitate ICTmediated research communication, for example, the Consultative Group Library Services Consortium (CGLISC), and CGIAR Virtual library. The consortium enabled member organisations to jointly subscribe to journals, while those who could not subscribe could be directed to libraries for the articles (see 5.3.1).

Other local, regional and international networks reported by participants to be promoting ICT-mediated research communication by acting as a mechanism for communicating research outputs include the KLA (Kenya Library Association), KENET (Kenya Education Network), the ASARECA (Association for Strengthening Agricultural Research in Eastern and Central Africa), IAALD (International Association of Agricultural Information Specialists), SCECSAL, IFLA and Friday coffee. KENET concerns itself with addressing ICT infrastructure issues in the universities whereas KLISC addresses content. The ASARECA is a regional network for Eastern and Central Africa for promoting agricultural research and extension.

Both librarians and researchers reported that regional and international networks have been instrumental in ICT adoption for communication of research. Library managers 1 and 2 gave the example of KLISC, which resulted from INASP, which in turn arose from IFLA. Such networks were said to give exposure to state of art technological development and thereby helped facilitate changes of attitude for those who participated in them, as library manager 1 explained:

So really the regional and international forums give us a lot of exposure on the state of the art as far as technological development is concerned and those who are able to attend those conferences come back with a different attitude altogether to ICT development. So I would say it has been of great advantage in adopting ICT. You know people go out there and say, "Hey, wait a minute! This is the way people are doing things".

It was reported that use of e-resources and e-communication in Kenya had come about as a result of participating in regional and international networks. Though scientists might not be connected in their institutions, they often had personal yahoo/hotmail email accounts that enabled them to access email outside research centres. Two 
research scientists, a lecturer and a communications official, indicated that such networks provided researchers with online linkages with counterparts and discipline experts abroad, in the US, Canada, and UK, and also provided opportunities for further training, seminars and conferences, thereby facilitating the diffusion of ICT.

Thus, networks/consortia were playing a vital role in ICT adoption, because most participants' discussions with colleagues were electronic, through email, D-groups, wiki, and/or blogs. Thus, there was web-based communication and transfer of knowledge within and across networks. The participants indicated that these networks were forcing researchers to adopt ICT, which in turn hastened and facilitated cumulative exchange of information as ICT became the means of communication, owing to its speed, cost effectiveness and ability to elicit immediate response. Hence, researchers have had to adopt ICT to be able to communicate because, as a librarian summed up, "when they tell you to go to the wiki if you don't go to wiki then you will not be able to see what the others are seeing or you will not be able to communicate with the others in the network" (L2_E).

Therefore, the findings affirm the diffusion literature indicating that networks and consortia groupings are important in the adoption of any new innovation (Vandooren 2002; Rogers 2003; Casal 2007). On the other hand, the findings also add another dimension: the idea of consortia as a means to facilitate a computer-mediated research communication mechanism that takes care of the end users at the grassroots. While the literature focuses on consortia related to professional groupings such as librarians and publishers, the findings of this study also called for consortia for specific target groups, such as flower farmers. Researchers could then work with these specialised groups in terms of communicating relevant research information. This would be unique in that it would refine the information dissemination needs of particular user groups to their unique socio-cultural context. Thus, the notion of social networks and consortia is context specific and no one size fits all.

\subsubsection{Traditional cultural values}

Further to the need for social networks, the findings established that traditional cultural values affected computer-mediated research communication. These included the communal/oral culture of Kenyans and education culture which will be discussed in the following sub-sections. 


\subsubsection{Kenya's communal/ oral culture}

Culturally, as argued by a senior government official, "Kenya, like many other African countries, is not a very technorate country in terms of if you are to put technology and IT on a scale against other things... or IT savvy country in terms of our national value systems. We see ourselves more on the side of a traditional hard paper society. We are more of an oral society...” (GO1_Ma). A researcher further maintained that the social/communal nature of Kenyan people, where most time was spent in social interactions, has hindered fast adoption of the computer-mediated communication:

Don't forget that actually many of us Kenyans we are very, very cultural. We are also very social and that social also extends to the wider community in terms of probably your time may be more needed, I'm looking at it in part of time, you may need to interact with so many people ... So you may find actually that wears you out first of all from the concentration and from actually the rigours of sitting ... being most of the time on computer (LR1_A).

As a senior scientist and a librarian explained, the computer-mediated ICT is new and unfamiliar and was viewed as private and anti-social, thus, cutting off the adopter from those around him/her. The scientist said this is unlike other ICTs such as radio or television, which had been around for a long time, were familiar and simple to use, and suited the social/communal culture in that people could interact and socialise with one another freely as they listened/watched. A library manager concurred with the view:

Then when you particularly get out of the urban area and you get into the rural areas again you get that fear of the technology trying to interfere with personal interactions. So again people are reluctant to go to technology because of that (L1_A).

The senior scientist above reported that user groups like farmers were more responsive to traditional communication channels, such as radio and public gatherings, than computer-mediated communication and expressed concern that such tools were not exploited in communicating agricultural information:

... when you look at tools that reach farmers, the tools that are most effective for communication such as radio, such as public gatherings where people show enthusiasm and appear are hardly exploited for agricultural development and yet we expect more sophisticated tools to be applied ... If such forums or tools are increasingly used to communicate agricultural messages, I think they will reach very far (RS1_G). 
This argument was endorsed by another scientist who felt that high dependence on the "Internet has a disadvantage of making you discount the aspect of face to face meetings and may be we have not reached a point where we can teleconference" (RS3_F). She felt that physical meetings were still crucial in communicating, in that agreements were reached faster and it was possible to accommodate new ideas that sprung up as discussion went on, unlike in online discussions. Face-to-face communication also allowed for non-verbal communication, like facial expressions and gestures, which are important in any interaction. Further, two researchers noted the fear of miscommunication associated with online as opposed to face-to-face communication:

... you can lose your information or end up sending it to somebody whom you were not intending if not very careful...(LR1_A),

and the fact that,

...you can easily be misunderstood over Internet. So you have to always choose your words, reread your mail... it is so easy to write and make mistakes... when I use Internet and assume I have done the right thing only to get a mail the next day asking me, “Did you really mean this?”... (RS4_F).

Thus, using the Internet required caution because unlike in oral communication where you could correct or clarify immediately, it took time to do so in online communication. The participants felt this could discourage Internet adoption in an oral society like Kenya.

A university ICT director commented that the social nature of Kenyans, where everything centres on the oral, explained the popularity and widespread adoption of the mobile phone. In contrast, computer communication requires users to work alone quietly, as well as demanding writing, reading and foreign language skills to be able to communicate:

... one of the reasons why mobile phones are really so popular is because our culture is more or less oral... we talked to this or this, you know sometimes back we never used to have anything written...So the mobile phone has really helped that because ... you can talk orally on it to somebody else as opposed to may be when you are using computers to just record that and probably read, may be store it somewhere. It was not so much of our culture. ... And when you tell about it in our culture, it is much more pleasing... the use of the mobile phone, I think that one has gone very well with our culture ... But if you just insist on only text, our country is not very strong simply on text (LR3_B).

The popularity of the mobile phone among African communities is also affirmed by a study undertaken in Burkina Faso (Hahn and Kibora 2008). 
Another scientist reported that the oral cultural tradition over the generations has also resulted in the lack of a reading culture in Kenya. This is counterproductive when it comes to extracting information in any computer-mediated channel:

The other thing is reading part of your culture because you can communicate what you want to communicate but to be communicated to you have to access this through reading? So basic principle of reading as a culture will impact on the amount of information and the benefit you are likely to get from ICT or Internet. So if you are not the reading type, if you are the type that the newspaper is the most important source of information, then I'm sorry as much as access may be improved you'll still be bound to only get the newspaper type of information (RS4_E).

There is need, therefore, for computer-mediated communication to capture the oral and the visual in order to be widely embraced in Kenya. If multimedia could be adapted to the oral culture of Kenyans, this would be a powerful way of communicating user groups farmers and others, as a senior lecturer and a scientist suggested:

But right now we have technology that is growing to capture even the audio and visual. You know if you are looking at agriculture you can capture images of plants and input that. I think it has more impact than just writing about it.... and the digital technology just needs to be improved to take care about the multimedia aspects, the issue of image, text, audio and video. So if we can enhance all the multimedia aspects then I think our culture may be taken care of and we are going to see more use of ICT (LR3_B)

I think communication today has to apply different set of tools. So ICT in this context is not just electronic systems (RS1_G).

A case in question is the example in Kenya discussed in the previous chapter (see 5.3.1) where flower farmers were successfully being served by one entomologist using a cell phone and computer-mediated technology to communicate. That way the farmers' problems could be addressed without the entomologist ever visiting the field:

But I know here in Kenya that we have farmers who are growing flowers and they are communicating electronically with experts. I know one entomologist who is able to solve entomological problems on the spot communicating through cell phone to a computer ... to serve a myriad of farmers because they can take a picture of what they are seeing on the field and can send it (RS1_G).

The idea of such a multimedia approach to ICT adoption is endorsed by Casal (2007).

Despite the negative influence of the oral and communal culture on adoption and use of ICT, a government official reported another aspect of Kenyans that was, nevertheless, ultimately helping foster ICT adoption. He explained that "Kenya is a very copycat country” (GO1_Ma) and Kenyans had a culture of wanting to look 
modern and move with the times, which led them to copy what was new "even when the concept has not really been fully understood” for fear of being left behind. The official further said it was this culture that had been the push behind adopting ICT, even in universities and government institutions which "bought computers that were just for typing. So we are very fast in copycatting” (GO1_Ma). Some participants added the mushrooming of Internet cafes in towns attested to this, although they were quick to note that the majority frequenting the cafes were the young, the so-called dotcom generation [which is true from the researcher's own experience as a resident and frequent user of the Internet cafes]. The impact of communal/oral culture was still prevalent among the old, as the findings established.

Borgman (2007) attests to the supremacy of face-to-face discussions and argues that ICT play only a complementary role to this. The same is echoed by Bowker (2005); Latour (1988); Livingstone (2003); Olson (2005); Olson \& Olson (2000); Sonnenwald (2007). Other studies have shown communal culture to have impact on the adoption of ICT (Goodman \& Green, 1992; Bagchi, 2001). Limo (2008) confirms the adverse effects of ICT on Kenyan communal family life when he argues that, "While the TV brings the family together, the computer is busy killing family life. Most communication technologies tend to reduce interaction among people in a given physical location while increasing interaction with those in the virtual space.”

\subsubsection{Education culture}

As well as communal/oral culture discussed in the previous section, some participants observed that traditional values and perspectives on education in Kenya have lessened ICT adoption and use for research and learning, because the education and ICT cultures conflict. One ICT lecturer indicated that the education curriculum in Kenya is teacher-centred; hence, promoting dependence on the part of the student. Another lecturer reported that this culture of dependence on the teacher tied with the traditional story-telling sessions where the young sat and listened as the elderly inculcated values. ICT-mediated communication, on the other hand, demands the learner takes an active part and collaborates in his/her learning, which has become a problem for Kenyan students and those who have gone through the education system. Given this pedagogical orientation, the ICT lecturer explained "even while given all these materials, the student may not have the necessary background to be able to milk the 
knowledge from the resources or use the resources effectively in getting the knowledge other than may be wishing there is actually an instructor there to guide them” (LR2_A).

The instructor-centred education culture in Kenya has resulted in a deeply engrained respect for the teacher, who is seen as author of knowledge and, therefore, a symbol of authority whose word should be respected and not challenged. As a result, the respect for authority created a dependence syndrome that in turn affected adoption of ICT for research/learning purposes because “...that thing of the respect- the respect for power... man, the lecturer is right. And I will always depend on the lecturer more than I will depend on my abilities to actually derive that information and knowledge from the material that is available,” (LR2_A)

The negative influence of culture on the adoption of ICT for teaching, learning and research supports earlier findings by Ondari-Okemwa (2002). He found differences in culture between collaborators from the West and Africa to be a barrier to virtual learning, as all were not on the same footing in issues of virtual learning. He argues that, "it is not very easy for a professor based in a university in North America or Europe to establish appropriate rapport with students in a Kenyan university setting. For one, a Kenyan student is surrounded by a different cultural environment as well as a different information environment," and concludes that "a professor is able to more easily establish rapport with students with whom he or she shares many cultural values” (p. 327). Moreover, he reinforces the view that pedagogical issues like teacher-centred learning affect ICT-mediated communication. He argues the fact that "a lecturer or a teacher is looked upon as an authority in his area of specialization" means "a teacher physically present in class can alter classroom behaviour selectively to the extent that certain technologies cannot help him/her solve certain important problems without seriously eroding his or her classroom authority” (Ondari-Okemwa, 2002, p. 326).

A senior scientist explained that educational culture is critical in ICT adoption and use, because our values are based on how we are educated. In Kenya the emphasis in education has been towards white collar jobs whose key attraction is an office with ICT accessories. The result has been an office-orientation culture where a computer is 
seen as a social status symbol, not as a tool for research or work processes; so, the young are not oriented towards making use of the computer as an enabling tool for work and research. This cultural orientation has also affected how the young use Internet. They use it to look for opportunities that will land them white collar jobs which in turn will give them the social status befitting such an office. They have not been socialised into ICT-mediated research, either for business ideas or opportunities, as one research scientist summed it thus, “... in terms of our cultural values ... I'm not sure of how many young people will look into the internet for business ideas, for business opportunities or for a market...” (RS3_F).

Nevertheless, the researcher above observed that the cultural orientation of an office culture with a computer as a status symbol might be changing, due to the demands of the external environment - the recognition that there were no jobs assured after school. She felt this might make the young use the Internet more for research but maintained, "there could be a change but it will be slow because our culture is basically driven by an office, the end product should be an office, to be sitting in an office space” (RS3_F). So, forces from the external environment may make Kenyans adopt the ICT for research. One participant reported slow cultural change was beginning to happen with the business community engaging in online car purchases. It could only be hoped this will spread to other professional communities.

On the other hand, there had been some enabling factors in the socio-cultural environment which were facilitating the rapid changes being witnessed in computermediated communication in Kenya. For instance, Kenyans'/Africans' general liking for what is imported and fashionable (see 5.2.1) had been an enabler to ICT adoption:

The environment is ahead of the legal process of reform and it is because African countries seem always to like these new fashionable imported concepts, including ICT, without subjecting and interrogating them to their situation (GO1_Ma)

It is something strange that Kenyans love anything foreign... so much that adopting to anything foreign has never been a problem in Kenya. ... We seem to be a sponge for foreign influence. ... The adoption of the comfort with technology for Kenyans is not a big issue. Kenyans love new things. They are crazy for new things. They are crazy for new technologies, they are crazy and they work with them as if they are fads, like you know it is the latest fashion in which there are even elements of competition who can take it up faster than the other. ... We will take up even if it is not good for us (LR2_A). 
Thus, as indicated by another senior government official, "Kenyans have very positive attitude towards ICT" and they "have no issues taking up technology" (GO2_Mb) as evidenced in the widespread adoption and use of the cell phone:

... it is known that even when the mobile was costing Kshs. 250,000 Kenyans had it. When the mobile has now become easily available, even the mama mboga [women selling vegetables] in the street, even the manamba [minibus conductors] and the water vendor have a phone. As for the uptake of new things/technologies, we are a sponge! (LR2_A).

Thus, the problem was reported to be lack of infrastructure, not the Kenyans themselves, as will be discussed in the next chapter.

\subsection{Chapter Summary}

The chapter has analysed and discussed findings related to the socio-cultural environment in ICT-mediated scientific research communication in Kenya. Table 6.1 gives a summary of the findings on the socio-cultural factors highlighted in this chapter.

Table 6.1: Summary of Findings on Socio-Cultural Factors

\begin{tabular}{|c|}
\hline Socio-cultural factors \\
\hline $\begin{array}{l}\text { - ICT appreciation } \\
\text { - } \quad \text { Lack of understanding } \\
\text { - } \quad \text { Fear } \\
\text { - Attitude of the research community } \\
\text { - Demographic factors } \\
\text { - Age/Level of qualification/Exposure } \\
\text { - Gender } \\
\text { - Comerty and literacy levels } \\
\text { - Traditional cultural values } \\
\text { - Communal/Oral culture of Kenyans } \\
\text { - Education culture. }\end{array}$ \\
\hline
\end{tabular}

The findings have revealed the importance of understanding the socio-cultural context because it influenced how ICT was perceived, adopted and used. In oral and social/communal societies like Kenya/Africa, ICT required a multimedia approach, in 
order to integrate well within the social-cultural norms of the people. It emerged that demographic factors such as gender, age, and poverty and literacy level affected ICTmediated research communication and would need to be addressed in order to increase ICT adoption and use. To increase ICT adoption and use, intensive and extensive IS/ICT campaigns would be important to create awareness about the role that ICT could play in the development process. An understanding of the potential and benefits of ICT could lead to its prioritisation in research budgetary planning to facilitate ICT-enabled research communication. Figure 6.1 reflects a revision of the social/cultural dimensions presented in the preliminary model.

Figure 6.1: Revision of the Socio-Cultural Dimensions
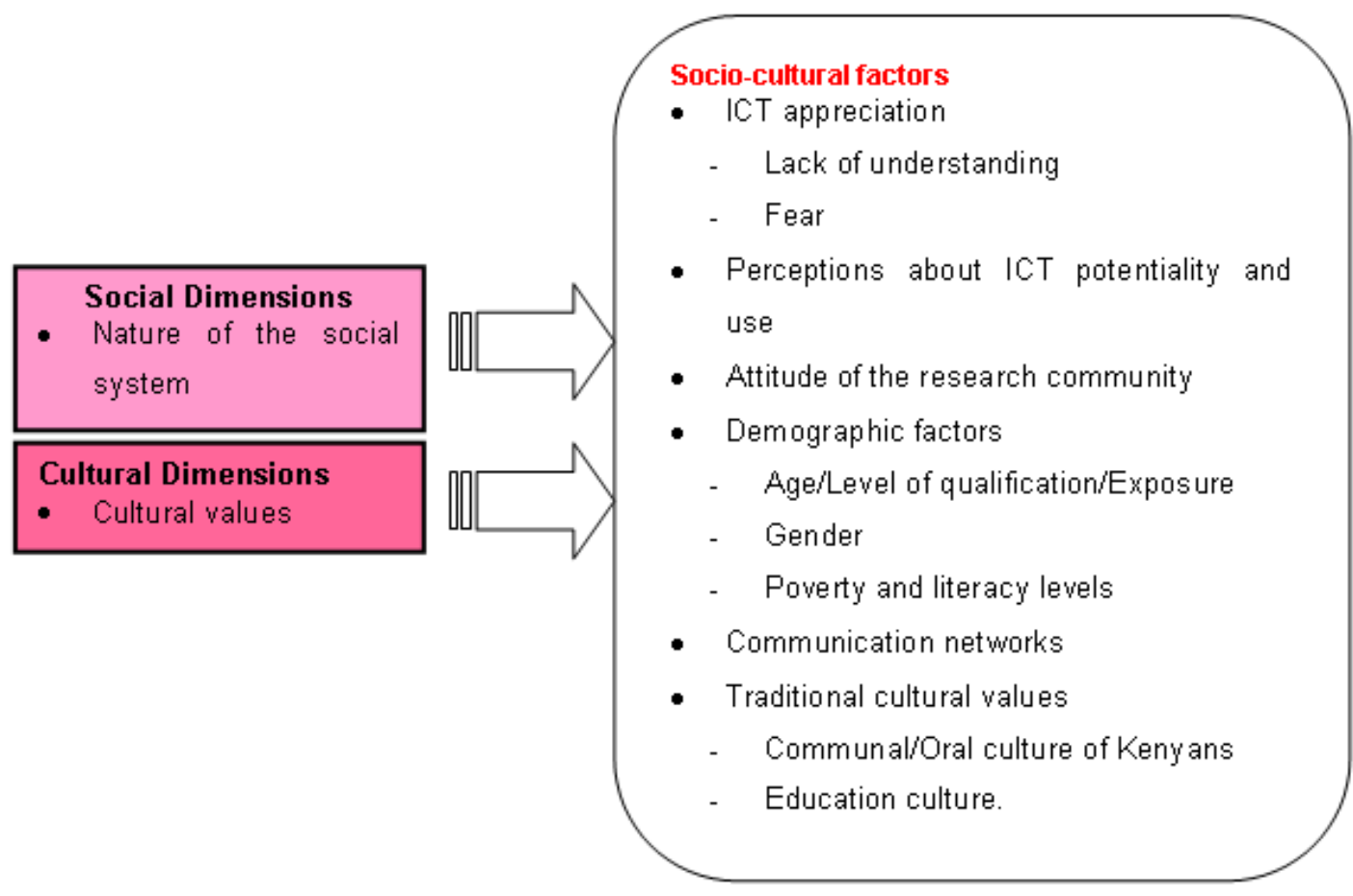

Figure 6.1: Revision of the socio-cultural dimensions

The preliminary model, informed by a thorough review of the diffusion theories, broadly showed that the social and cultural environment affected the diffusion of any new innovation (Hofstede, 2001; Rogers, 2003). The theories comprised a general and broad statement about what affects the adoption of any new innovation. Also, they suggest a separation of the social and the cultural because, apart from being developed by different individuals at different times, they were also based on the experiences 
from the North where there tends to a marked difference between the social and cultural.

On the other hand, the findings in this chapter, though supporting the theories, presented a specific contextual analysis and illustration of the effects of the Kenya socio-cultural environment on the adoption of ICT for communication of research outputs by researchers in the applied sciences institutions. The findings identified distinctive features characteristic of researchers in the Kenyan context such as ICT appreciation and perception; attitude of the research community; unique demographic information on age and level of qualification, gender and poverty and literacy levels; communication networks and traditional cultural values like orature, communalism and education culture. Here, the social and the cultural are combined because, as already explained, the social merges into the cultural and vice versa.

The next chapter presents and discusses findings on factors of the institutional environment that affect ICT-enabled research communication. 


\section{CHAPTER SEVEN}

\section{THE INSTITUTIONAL ENVIRONMENT}

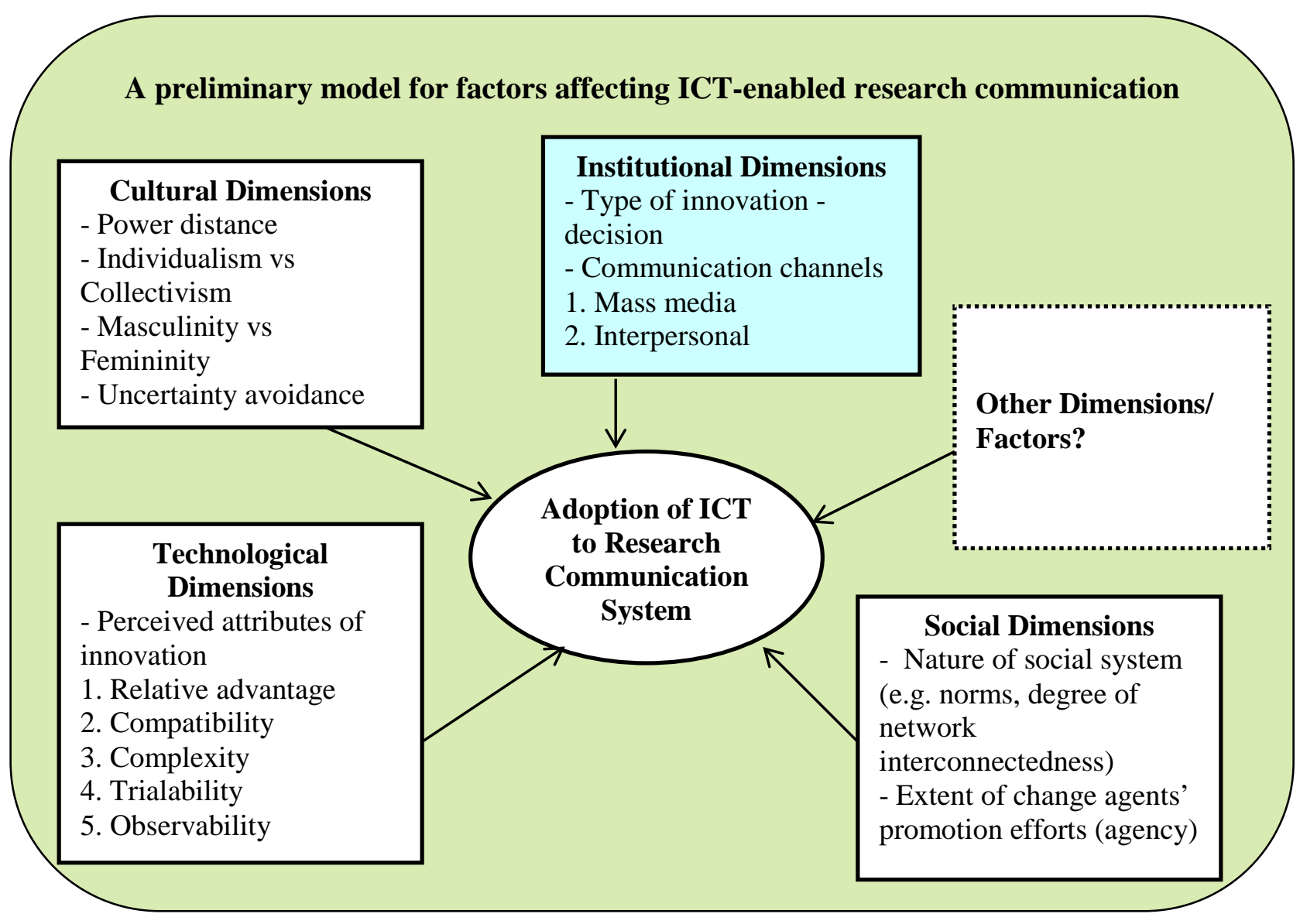

\subsection{Chapter Overview}

The study sought to establish the barriers and enablers to ICT-enabled research communication by researchers in Kenya. The previous chapter has looked into the socio-cultural factors affecting ICT-mediated research communication. This chapter presents findings related to both the external and internal organisational environments in ICT-mediated research communication.

Studies and theories informing the theoretical framework of this study stressed the need for an analysis of the organisational environment, because it affects the adoption and use of ICT (Hofstede, 2001; Oyomno, 2006; Bagchi\& Udo, 2007; Bakkabulindi, et al., 2008). The findings in this study identified organisational barriers to the adoption of ICT such as ICT governance; capacity for ICT-mediated research 
communication; demand for research/teaching management information system and research resources. The subsequent subsections discuss these factors.

\subsection{Institutional factors}

\subsubsection{ICT governance}

The important role of top leadership/management in innovations diffusion is emphasised in empirical studies (Damanpour \& Schneider, 2006, 2009; Elenkov \& Manev, 2005; Moon, 2000; Moon \& Bretschneider, 2002; Thong \& Yap, 1995). Rogers (1995, 2003) regards the type of innovation-decisions and communication channels that create awareness about innovation as being factors influencing the adoption of any new innovation. These issues relate to governance, which, as Oyomno (2006) says, “defines the framework within which stakeholders play complementary roles in the development of a sustainable national ICT capability” (p. 102). He says governance aspects such as ICT leadership, and institutional, policy and legal and regulatory frameworks are important in the development of such capability (Oyomno 2006). The IT Governance Institute ${ }^{5}$ (2003) of UK stresses the same and sees IT governance as being integral to enterprise governance. It views IT governance as consisting of the "leadership and organizational structures and processes that ensure that the enterprise's IT sustains and extends the enterprise's strategies and objectives” (p. 11). It adds that "effective communication among all parties based on constructive relationships, a common language and a shared commitment to addressing the issues” (p. 11) are critical to the success of these structures and processes. IT governance directs IT endeavours to ensure IT performance aligns with the enterprise for realisation of the promised benefits; IT use enables the enterprise by exploiting opportunities and maximising benefits, while ensuring responsible use of IT resources and appropriate management of IT-related risks (IT Governance Institute 2003). Thus, IT governance provides the initial direction upon which "a continuous loop is established for measuring performance, comparing to objectives, and resulting in the redirection of activities where necessary and a change of objectives where appropriate” (IT Governance Institute 2003, p. 11).

The findings in chapter 5 revealed the need to reinforce the requirement to do and communicate research and also to provide a mechanism for research communication

\footnotetext{
${ }^{5}$ The UK’s leading provider of expert services for IT professionals (http://www.itgi.org)
} 
(5.2 and 5.3 respectively). These needs called for institutional and strategic policy and legal and regulatory frameworks, in addition to visionary leadership which were reported to be lacking (see 5.2.2, 5.2.3 and 5.3). It also emerged that the priority needs affecting researchers' communication of research outputs also affected their adoption of ICT for research communication.

The findings in this chapter reveal the sub-factors relating to ICT governance such as political and institutional leadership; institutional framework; policy and strategy and legislation and regulatory framework, and control over mass media channels.

\subsubsection{Political and institutional leadership and culture}

Chapters 5 and 6 identified a gap in the Kenyan political and institutional leadership that negatively affected the country's scientific research communication to the extent that there was no research taking place (see 5.2.1). Owing to non-visionary leadership, it appeared that a research culture had not been engrafted in research institutions in Kenya (see 5.2.1 and 5.2.3.3). The same leadership and cultural factors affecting research communication also affected the adoption of ICT. The findings indicated that the leadership lacked an appreciation of ICT in government functions and clear perceptions of its functionality. This lack of vision was also found to be a hindrance to ICT's implementation for research communication (see 6.1.1 and 6.1.2).

The literature affirms the need for effective leadership in the implementation of ICT (Moon \& Bretschneider, 2002; Nystrom, Ramamurthy \& Alla, 2002; Oyomno, 2006; Thong \& Yap, 1995). Oyomno (2006) underscores the centrality of leadership in the building of an information society enabled by ICT. He views it as "an overarching function that provides the championship, direction, and coordination required to create a national vision, policy, strategy, architecture and standard for ICT development and deployment in government and in the economy” (p. 103). The IT Governance Institute (2003) confirms the same, "IT governance, like other governance subjects, is the responsibility of the board and executives” (p. 11).

\subsubsection{Institutional framework}

Oyomno (2006) sees institutional framework as key to "the seamless flow of information and knowledge and the engagement and involvement of a broad spectrum of stakeholders” (p. 104). He explains that some of the national digital indicators in 
Kenya include current institutional arrangements in government and the extent to which they promote integrated development and the delivery of government services; new advisory bodies that bring stakeholders in each sector; continuous review and appraisal of existing organisational arrangements; and formulation of new institutional models and realignments that minimise waste and related costs.

The data revealed the lack of a clear institutional framework to guide ICT-mediated research communication implementation within and among science research institutions in Kenya. It seemed that there were no mechanisms for integrating ICT into work routines in most public research institutions in Kenya. One respondent said that "... we have not reached the level of integrating ICTs into our day to day work" (L2_E). This idea was reinforced by a lecturer who commented that researchers and students were not required to use ICT in their communication and they "do not have (up to now) to actually use ICTs to meet or solve their problems and challenges or to use them effectively” (LR2_A). He explained that they [researchers and students] use ICT mainly for personal and office automation functions like word processing, calculations, sending emails, reading newspapers, but not for research or e-learning.

A library manager also reported the non-integration of existing ICT units that were intended to provide support to the researchers. She explained the units were seen as independent of the departments whose functions they were meant to support:

Most of the organisations are still looking at ICTs as that department and then we [other departments of the organisation] are here (L1_A).

[The researcher observed this gap as she moved around institutions and ministries, explaining the focus of her research. They would send her directly to the ICT department, even though she tried to explain that the ICT staff might not be able to give her the information she required. It was the researcher, administrator, editor and librarian who were closer to what the researcher was doing, not the technical person].

Participants also made the observation that ICT had not permeated into the rural areas, where majority of the target groups for research outputs resided, neither was there any effort to link research institutions with the user communities: 
I've not seen an effort to ... linking the School of Medicine with the community as a way of health information ... using computers... we have not handled that aspect as a university (LR1_A).

How can we add value to that access to make your brother get that information, to make your other community member use the same facility to do it? (RS4_E).

Researchers stressed the need for ICT to be integrated at the community level, if their research outputs were going to have an impact. They felt it was "futile" for researchers to have access to ICT if they could not reach their clients to disseminate their research outputs, as noted by a research scientist:

... even if $\mathrm{C}$ connects all their centres when our farmers, clients and partners are not capacitated and connected, it will be futile. The scientists will get the technologies but for the technologies to be absorbed the way I would have wished, even our clients and partners need to be connected or need to be capacitated in ICT. For us to achieve these goals that I'm referring to there is need for all the people in the agricultural sector to... be able to access and exchange the information... (RS6_C).

A lecturer cum ICT director suggested that researchers and scholars "should be compelled to [use] ICT to disseminate this information” (LR3_B). Nevertheless, he underscored the need to "provide the framework" on which to base ICT adoption and integration both nationally and institutionally across the board, to ensure the research institutions could be accessed by all their clientele and others who needed to use their services.

The non-integrated application of ICT in research institutions did not reflect the common goal of a scholarly cyberinfrastructure, which Borgman (2007) says should be "for the components to work together despite the variety of providers, users, and purposes” (p. 254). The same views are shared by others in the literature (Mutula, 2001; Ondari-Okemwa, 2002; Van de Sompel, et al., 2004; Oyelaran-Oyeyinka and Lal, 2005; Gebremichael and Jackson, 2006; Casal, 2007).

To facilitate integration of ICT across the board, a library manager suggested "the concept of the information kiosk can work once the infrastructure is provided and even our farmers can go and find details about prices of their commodities” (L2_E). Another librarian talked of “telecentres” (L1_A) as possible channels for user groups to communicate with researchers, while a senior research scientist called for 
"information nodes at the grassroots level" ${ }^{6}$ (RS4_E), which could help the user clients and the researchers communicate with one another; thereby, helping address problems in a timely manner. These initiatives would facilitate access to new agricultural and health technologies and methodologies as well, thus, acting as possible information inlets and outlets for information exchange.

The concept of information kiosks, which has worked in such developing countries as India (Kumar, 2001), was seen as a viable solution to the problem of integrating ICT across the board, if government intervention provided the necessary infrastructure at grassroots level. This would contribute towards informed agricultural and health practices, which could ultimately improve people's livelihoods and health issues as per Kenya Vision 2030 (Government of Kenya ,2007) goals and objectives and also the 8 UN Millennium Development Goals of alleviating poverty and hunger, health related issues, through to developing global partnership for development (see http://www.un.org/millenniumgoals/goals.html).

As well as non-integration of ICT, respondents indicated that the lack of an institutional framework for ICT implementation had resulted in wrong focus. They said those in authority tended to focus on infrastructure, tools and ICT personnel, ignoring content, communication systems and/or mechanisms for communicating that content - what one government research communications advisor called the " $T$ " [technology] at the expense of the "I\&C" [information and communication] (GO1_Ma).

Consequently, participants felt recognition was given to the computer experts (those who could manipulate the tools) rather than those who produced and managed the content, like the researchers and information professionals working with them. The tendency, therefore, had been to follow the computer experts' advice, which resulted in the buying of computers and software without considering their application. This resulted in what one library manager called "empire building” where the focus was on:

\footnotetext{
${ }^{6}$ The contextual interpretation of the three different terminologies - "information kiosk", "telecentres" and "information nodes"- which respondents used interchangeably, is public places connected to the Internet, which users and scientists in disadvantaged places could visit to send and access information.
} 
- employing ICT technicians, without regard to content professionals like administrators, researchers, librarians and technologists

- ICT infrastructure, like student computer laboratories and stand-alone computers and accessories, without the necessary content that could facilitate communication.

One scientist described this as "putting the cart before the horse" (RS1_G); thereby, resulting in no outputs to communicate, as reflected by the thin content that characterised many public institutions' websites. As a result, students were reported as downloading music while researchers did their own thing. Thus, as a librarian and a lecturer indicated, "a link is missing” between the tool and content the tool should relay, which needs bridging to facilitate ICT-enabled research communication.

A library manager commented that this contrasted with practice in the developed Western world where ICT were only viewed as tools to manage content and processes, and their acquisition was predicated on this:

I was recently in Addis Ababa coordinating and the forum was giving so much publicity to ICT people and they forgot; they didn't even want to know the librarians had gone there, we had various people and geographic information systems - these people were totally ignored. The focus was given to ICT infrastructure. And we were there trying to shout what matters is the content, not the tools. So you can see Africa is still going that way... I have attended several forums in Europe, in the US and you find that what they are focusing on is the content ... They talk software and not what and how the software can help them do in work places (L1_A).

There was also a difference in emphasis between the international institutions visited during the research and the national public institutions. These Western-oriented institutions viewed ICT as a facilitating tool and had put in place institutional frameworks to take advantage of the new technology. A participant from one international institution reported that their institutional environment "is a technological kind of setting where the technology is actually valued and has to be used. The systems have been put in place” (L4_G). Another participant added failing to use ICT "would be so negative to you, yourself, that it is not worth trying to do that, and in any case as part of the recruitment that [ICT] is given a lot of emphasis and there is also room for capacity enhancement” (RS4_E). Thus, ICT in these institutions was valued and used and the cultural diversity resulting from the multicultural environment within these institutions has influenced those people from non-ICT backgrounds to adopt and use ICT; hence, enforcing ICT adoption. These 
institutions emphasised computer-mediated research communication by all their staff for enhanced efficiency. As such, all departments worked in consultation and everybody was required to be ICT compliant to ensure content was produced and delivered as required.

Nevertheless, participants in the three international institutes in the study lamented that they had little impact on society, despite having good ICT-enabled communication systems with clear content, because they had to work within the unaligned national systems to disseminate their outputs. There was need for an institutional framework to refocus ICT implementation within the national system in order to ensure its integration across the various departments and units to facilitate production and dissemination of research content.

The issue of focus in relation to the implementation of ICT has been discussed by Casal (2007), who stresses that human development, in other words, people, not technology itself, should guide the design and evaluation of ICT programmes. Simborg (2008) and Berner, Detmer, \& Simborg (2005) call for rethinking concerning adoption of electronic health records (EHRs) system in America. Simborg (2008) argues that "adoption, per se, is not the goal. We must focus, in addition, on correcting the problems in EHRs...even at the risk of delaying adoption” (p. 129). Thus, the focus should be on the problem the computer-mediated information systems are to solve.

The findings of this study also mirror Hofstede's (2001) idea of high and low power dimensions owing to cultural differences within organisations. The national research institutions in Kenya had a high power distance and so tended to emphasise acquisition of computers and accessories as status symbols. This was also manifested in the high importance given to computer experts over content producers and managers. As discussed in chapter 5.2.1, some participants reported that it was a cultural norm for Kenyans to value and emphasise certain professions as more prestigious than being a researcher. Participants felt this could have slowed the adoption of ICT for scientific research communication in Kenya. This contrasts sharply with low power distance cultures in the developed world and in Kenyan international institutions which are aligned to the West, whose focus is on using ICT 
to enhance achievement of their goals; so, adoption of ICT in these institutions has been higher and faster.

\subsubsection{Policy, strategy, legislation and regulatory frameworks}

As well as institutional framework, there was need to understand the impact of government policy regulation and legislation on scholarly communication infrastructure, because research communication relies on government for funding and direction. Borgman (2007) confirms the importance of this when she says, "Scholarly infrastructure must be understood in the context of legal, policy and economic arrangements,” (p. xviii). Oyomno (2006) argues that policy is an important dimension of a national ICT environment for it provides a roadmap for the development of ICT capabilities and denotes an understanding by government about the role of ICT in the society. He explains that an ICT policy "is designed to strengthen the information and communication infrastructure, and address information technology, telecommunication, and postal services, among others,” (p. 105).

The findings of this study revealed that lack of or poor government policy and strategy and legislation framework has been a barrier to adoption of ICT in Kenya (see chapter 6.1.1). A senior government official reported that long -standing government monopoly of the communication system in Kenya had resulted in there being only one ISP, Jambonet, which he described as "a king of information such that no Internet would come to Kenya without passing through Jambonet” (GO2_Mb). The lack of competition ensuing from this monopoly meant Internet access was quite expensive and, therefore, out of reach for many. Telephone and fax have tended to the most common communication tools in most public institutions. The government official said that, from the 1990s to early 2003, there had been considerable government control on information communicated via the mass media, which had limited both communication and the development of communication networks. It also hindered the adoption and use of any ICT for dissemination of government information, which included research information.

The absence of any ICT policy regulatory framework to govern ICT implementation both at the national and institutional level has also been affirmed in the literature (Mutula, 2001; Bowman-Ngaruiya and Waema 2006; Ndede-Amadi, 2006; Sihanya and Odek, 2006; Odero-Musakali and Mutula, 2007). 
Participants also reported stiff bureaucracy as a barrier to ICT-mediated communication of government information. A senior government officer indicated that juniors had to be approved by their seniors, who also had the only available computers, to be able to communicate:

And the boss has the machine but he doesn't use it. What about the juniors? The juniors could be a dotcom generation; they could be using the machine alright, but then come to communicate that information out - now before you communicate out official information for that matter in the government, you have to have the sanction of a sultan like me. ...So it comes to me and I tell you, "Whom do you think is the boss here? Are you the boss?" (GO2_Mb).

Mutula (2001) and Ondari-Okemwa (2002) affirm the suffocating effect of bureaucracy on ICT adoption in Kenya research institutions.

In addition to bureaucracy, owing to the Official Secrets Act (Cap 187) (Government of Kenya, 1968), most government information was treated as confidential. Consequently, it was "classified; it is touchy," as two senior government officials reported. This limited free circulation, whether online or physical, which could explain the slow adoption of ICT for communication in government research institutions. The restrictive government regulation requiring clearance before one could communicate government information led to a situation of uncertainty avoidance (Hofstede, 2001), for people did not want to be caught on the wrong side of the law. This meant that one could not communicate government information freely, even with ICT.

The findings pointed to the need for repealing such legislation and policy, in order to guide communication of ICT-enabled government information (see also chapter 6.1). Hand in hand was a need for government to rethink, and prioritise ICT for research communication in its budget allocations, as a senior scientist suggested:

The government has to think twice about how to allocate funds to facilitate acquisition of what is considered to be the best tools to facilitate research [-] ICT. Money has to be there unlike what has been the case in the past (RS2_D).

Another researcher underscored the need for the government to move beyond planning, to ensure ICT-mediated communication projects are implemented appropriately. This researcher expressed an opinion shared by others that it was not lack of resources or planning that was really the problem in ICT-mediated 
communication in Kenya, but lack of professionalism in implementation of government plans:

R. What explains these [ICT] challenges?

P. I think professionalism. It is not poverty. I think Kenya has adequate resources; we only need to plan better but also spend less time planning and more time executing the plans appropriately, and I stress the word appropriate because Kenya is full of good excellent plans but poorly executed (RS4_E).

A senior government official observed that the laws and procedures governing communication in the public sector were outdated and did not recognise digital communication, including email. This could be a barrier to ICT adoption in government institutions’ communication processes.

The same is echoed by Wafula and Etta (2006), who call for "the need for inclusion of policies statements on the management of electronic records in national ICT policies” (p. 12).

A senior lecturer and ICT director reported that the government lacked enabling structures like an ICT Authority, such as the Rwandan Information Technology Authority (RITA), which could oversee and spearhead ICT implementation. He observed that Rwanda, which was still recovering from war, seemed to have moved faster in ICT implementation than Kenya because of the presence of such structures. He attributed this to the fact that those who were supposed to enact laws, the Parliamentarians, did not see ICT as a priority and also feared ICT might empower their constituents. He argued that, although an ICT Bill had been recently passed in Kenya (Government of Kenya 2007), an ICT authority was likely to be more forceful in ICT implementation. He expressed the need for key government ministries to take an interest in the ICT infrastructure and also to have champions in parliament to lobby for ICT (as, for example, one MP fronted for gender rights). This should be in addition to building ICT awareness and the capacity of the parliamentarians to champion such legislation.

Kalambuka (2008) affirms the lack of proactivity and involvement by the Kenyan parliament in addressing science and technology matters: "OUR PARLIAMENT is not evolving to deal with such a complex array of problems, processes and new developments. We have no specific structure for addressing science and technology - 
i.e. a complete lack of any structural parliamentary recognition of the area” (n.p). He suggests “a committee on science and technology would enhance the country's capacity to handle policy issues related to science and technology innovation” (n.p). This was also emphasised in the East/North Africa Regional Science Technology and Innovation Policy Reviews Workshop in Mombasa 30 March - 3 April 2009 (UNESCO 2009)

Sector-wise, there was need for ICT policies to guide ICT-mediated communication. For example, a senior scientist suggested, "there is need for all the people in the agricultural sector to have an ICT policy” (RS6_C). An ICT lecturer cum manager pointed to the lack of institutional policies and frameworks regarding adoption and use of ICT for teaching and research communication:

There isn't even a policy framework by the university to go towards e-learning... if the university had the necessary framework in the form of policy guidelines and also taking up as very strong strategic objective, then it would be possible to accelerate the upload or content development and populate the system and also start applying it officially in teaching and such (LR2_A).

The findings uphold Mutula's (2001) call for a policy framework and clear strategies by research institutions, pegged on the institutions' vision, mission and mandates, to guide and enforce the implementation of ICT-mediated communication. OndariOkemwa (2002), drawing on his findings of the AVU, stresses the need for "a shared purpose or vision" arguing that it "serves as a 'glue' of the virtual organization” (p. 325). Similar views calling for an ICT adoption framework in Kenya are shared by Wafula and Etta (2006) and Oyomno (2006). Kaniki (2004) also stresses the need for an STI policy to address issues pertaining to scientific communication in African countries.

Despite the government's seemingly dismal past performance in creating a favourable policy environment for ICT implementation, a senior government official reported that there had been, nevertheless, an attitudinal change that was triggering more ICT adoption within and across sectors. The government recognised the need for computer-mediated communication as a means to achieving its developmental goals as outlined in the Kenya Vision 2030 (Government of Kenya, 2007) and other earlier policy documents. It had begun introducing policies to facilitate faster diffusion of 
ICT within and across sectors, for example, the waiving of tax on computers and their accessories.

Moreover, the Kenyan government has also spearheaded development of the egovernment Strategy (2004); establishment of the Ministry of Information and Communication (MIC) in 2004 and the Ministry of Higher Education and Science and Technology (2006) to address science, technology and innovation (STI) issues. The latter ministry has so far developed an STI policy to guide ICT-mediated scientific communication (Government of Kenya, 2007).

\subsubsection{Control over mass communication channels}

In addition to institutional and policy and strategy and legislation and regulatory frameworks, as discussed under 7.1.1, effective communication among all parties within an enterprise is crucial if IT is to sustain and extend the enterprise's strategies and objectives (IT Governance Institute 2003). Effective communication denotes free flow of information to those who need it over the existing mass communication channels (Rogers 2003). For example, because Kenya's economy is largely agricultural, participants felt ICT-mediated communication could help inform agricultural practice. However, as reported by a senior scientist who was an agricultural expert in the African region, ICT-mediated communication is controlled by the educated whose culture was quite different from people in the farming sector:

So you see our communication tools are dominated by a very different culture than those who want to develop from agriculture...the majority out there in the rural areas (RS1_G).

He explained the elite are the people who already "have power in having knowledge and it is going to reinforce power to those who already have it," not the farmer or the ordinary person at the grassroots who practises agriculture. This view was supported by a library manager and a senior lecturer who argued that even the predominant language of the Internet, English, tended to favour the already knowledgeable urbanite in the cities, rather than the disadvantaged rural farming community where the majority are not literate. They explained that the urbanites are also the people with resources to afford computer-mediated communication; hence, the big digital divide between the cities and the poverty-ridden Kenyan villages. 
This could be an explanation for the slow penetration of ICT in Kenya, for the majority of the population resides in the rural areas of Kenya where ICT technology is yet to be felt. Those in the rural villages might not even know what a computer is nor did they have access to telephone system. In addition to their low literacy levels, they have not been sensitised about computer-mediated communication, and lack awareness of what the computer can do. Participants were of the opinion that with the widespread unawareness about ICT-mediated communication channels, it would take a long time for ICT to impact on the lives of the majority in Kenya:

The only limitation of these ICT ... is that it will work for the educated but what happens to the grassroots? We still have our social problems at the grassroots level and I think we still have to do some work to reach the grassroots in terms of taking the ICT to the grassroots (RS6_C).

This calls for mechanisms to be put in place to ensure ICT reaches those who need to have research outputs communicated.

Moreover, the interests of the two groups are totally different and this has led to the development of two parallel cultures - city and village/rural. This has affected the content that is delivered through the existing ICT-mediated communication channels like the radio and the TV. A senior agricultural scientist pointed out the city controls the communication channels and so "they drive the media; they drive the content of the media!” (RS1_G). He explained much of the content broadcast over the channels has been what met the needs of the urbanites, for example, foreign entertainment programmes such as football, music and politics. There was hardly any content or programmes for the ordinary citizen and farmer in the village.

This brings about the notion of “centres vs periphery” (Mazrui ,1978; Zhong ,1993; Altbach, 1996; Canagarajah, 1996; Muthayan, 2004) discussed earlier in the literature review (see 2.4.2.3) in ICT-enabled research communication. The cities are the centre and control what is communicated over mass media channels, while the rural areas remain in the periphery and are, therefore, powerless/voiceless over what is aired. It is a form of power distance (Hofstede 2001), where the elite call the shots in ICTmediated information dissemination. This raises the necessity for the various stakeholders to come together and devise strategies that can help ICT-enabled 
dissemination of research information to inform the developmental priorities of the country in line with the Kenya Vision 2030 and the MDGs.

\subsubsection{Capacity for ICT-mediated research communication}

"It is not enough for organisations to have access to technology or to be able to acquire technology. It is not enough for organisations to have information and data assets, whether internally or externally generated. They should have the capacity to use all of the technology and information resources to their own advantage," (Avgerou 2004, p. 41).

This sub-section will address factors related to researchers' capacity to engage in ICTmediated research communication. These include issues to do with human capital such as ICT awareness and skills and communication skills; lack of researchers' needs and institutional e-readiness assessment; ICT tools and infrastructure; content and applications and funding for sustainable ICT implementation.

\subsubsection{Human capital}

Human capital is a requisite national capability and an essential determinant of the capacity of any country to effectively and sustainably develop and use its ICT assets (Oyomno 2006). The findings revealed that Kenyans' lack of ICT awareness and skills and lack of communication skills were a barrier to ICT-enabled research communication.

\section{Lack of ICT awareness and skills}

The literature reviewed points to the need for adequate exposure to any technology to reach the threshold level for maximum use (Rogers, 1995, 2003). ICT-mediated communication requires skill, awareness and exposure in order for users to be able to communicate proficiently (Bagchi \& Udo, 2007; Casal, 2007 Durrant, 2004; Mbarika, et al., 2002; Muinde, 2004). Every participant in this study reported lack of or inadequate hands-on ICT skills and exposure as a major barrier to ICT-mediated research communication in Kenya. The majority of the researchers, unless they had studied in the developed world or were working in international research institutions or donor-funded projects, lacked training in and exposure to ICT and were just being introduced to the technology. Therefore, their ICT competence was inadequate to allow them to take advantage of the technology, as some participants indicated:

Our universities have not effectively invested in the human capacity to run those things [ICT] (GO1_Ma) 
A challenge that I have experienced in university A and I guess it is also elsewhere is that our people have not been well trained in the use of ICTs. The skills are lacking... we have not been able to sensitise and go beyond sensitising to kind of impart the skills to our staff and students to be able to effectively exploit ICT... (LR2_A)

... People are not moving rapidly to take advantage of ICT to do their research and this has been an impediment for ICT-mediated research because they are not competent enough to use ICT... (RS5_D).

This was aggravated by the fact that ICT support staff was, as a scientist reported, qualified in paper but lacked hands-on computer skills and motivation; so, they could not deliver the support they were supposed to provide to researchers.

Two respondents added that researchers lacked typing skills, which limited the proficiency with which they could use the computer to communicate:

I think the problem is how to handle the keyboard (LR2_A)

If, for example, the culture is such that we were never taught how to type, a simple thing, it will limit the speed with which you can communicate information to others and it will bog you down and a thing that should take you 5 minutes may take you 20 minutes (RS4_E).

A government official explained that lack of computer skills had been so bad when computers were being introduced in Kenya that many national institutions locked up donated computers for lack of skilled personnel to operate them:

...nobody was able to connect them [computers] because again, as I told you earlier, you purchase this hardware without [the] corresponding investment in training (GO1_Ma).

Two researchers reported that most researchers were not familiar with ecommunication like e-publishing and e-learning, and preferred the traditional communication system:

Actually things like sending information or even a publication to submit any information to journals these days I do actually send it online direct but for many of us it is not the norm. There are just very few people. You find that they are actually packing it and sending it through the DHL system or courier services (LR1_A)

Although e-learning is not an aspect of research as such, a student here at A university has developed an e-learning software called Wed-Soft ...to capture lecture notes. Before we capture the notes we have to train the lecturers so that they format the lecture notes into a format that is acceptable in the system (LR2_A).

They said there was need to build researchers' capacity on how to manipulate publishing software and functions like Photoshop. This would make it possible to anticipate the likely end product and enable them to collect photos and materials that would aid repackaging of their research outputs into formats that could be easily put 
online. Some participants said this called for strengthening of the IT departments or specialised professionals to assist researchers in synthesising materials for online communication.

A scientist indicated many researchers were also unaware of the free software available that they could use in their work and tended to rely on the Microsoft packages bought along with the computer. There was need for awareness creation on the ICT that were freely available, which affirms Durrat's (2004) recommendation that there was need for specific open access training.

The lack of computer literacy and proficiency in use among the majority of staff in public institutions contrasted sharply with the ICT competence of staff in the international research institutions where, as a library manager reported, “... [the] majority, especially the scientists, have come from the developed world and already have had the exposure to technology and they are able to transfer that knowledge to the staff around them; so, most of them are confident in the use of the technology" (L2_E). [The researcher also observed that those in the national institutions who had trained overseas did not have any problem with ICT use]. This spells the need for ICT training in public institutions.

Nevertheless, one exception was noted in that researchers from both the international and national research institutions lacked knowledge of cost effective Internet information search skills. A lecturer observed that,

... even those who have laptops and PCs there are issues that probably they need to learn... when it comes to content on the Internet, where you can get it and what you should do with it... (LR3_B).

A librarian commented the majority of the researchers just knew how to "google and google and that's it” (L2_E) and so when "you sent them a link you may still find some of them coming back to tell you they cannot get these things.” She called for library user education on specialised search skills, such as Boolean logic, so that researchers may be able to exploit all avenues.

These findings point to the need to equip researchers, scholars and ICT support staff in public research institutions with ICT skills to facilitate ICT adoption across the board, as a research communications official, as observed some participants: 
It is not enough in my view to recommend that you'll give every professor at the university a computer. In fact it is more important to invest in retraining them ... you also have to re-orientate the people to become users and consumers of this ICT (GO1_Ma)

... even by the time you are getting on to putting your work on an Internet platform you have to have been good to start off with or you have to get help from people who are good or have a department that can help you put that work on (RS3_F).

The findings confirmed Rogers' (2003) theory that there is need for threshold exposure in adoption of new innovations and Rogers, Takegami and Yin's (2001) argument that the process of technology transfer is a difficult type of communication that demands trained and skilled personnel. The findings also aligned with other studies on diffusion of ICT in Kenya and other Sub-Saharan African countries, which call for ICT awareness creation and training (Mutula 2001; Mbarika, et al; OndariOkemwa 2002; Oyelaran-Oyeyinka and Adeya 2002; Muinde, 2004; OyelaranOyeyinka \& Lal 2005; Wafula \& Etta 2006; Bagchi \& Udo 2007; Odero-Musakali \& Mutula 2007).

\section{Lack of communication skills}

As discussed in chapter 5 , the ability to originate and communicate a message is the key thing in research communication. ICT is a tool whose application for communication of research outputs is predicated on other skills and abilities. Researchers need to be able to create research outputs that can be communicated via ICT. However, the findings revealed a gap in the capacity of the researchers and scholars to communicate research outputs, because they lacked communication skills (see 5.4), and, accordingly, were said to be poor communicators who were not trained to communicate. They also lacked the capacity to repackage technical outputs into formats and language that could be easily understood by and communicated to end users, who are supposed to make use of research outputs to improve the quality of their lives (see 5.4). The lack of communication skills was also affecting ICT-enabled research communication, as a scientist observed:

... if you are not good in writing and communicating your ideas in writing then the effectiveness and impact to ICT will still suffer from that inadequacy (RS4_E)

Some participants reported that communication in many government departments, including research institutions, had been left to IT personnel who themselves were not schooled to communicate content (see also 7.1.1.2) and, therefore, could not assist scientists in making required content available: 
... the IT personnel are technicians who were not trained for example to process information. They can't do it [communicate] ... ICT personnel are invariably not trained in communication for example to fully utilise ICT in government you not only need technicians- people only schooled in soft/hardware $(\mathrm{T})$ but also the I\&C personnel, which is very scarce, people who can translate information into usable quantities, people who can edit and make some sense of judgement (GO1_Ma).

Thus, one needed to be a good communicator first to be aided by ICT. A librarian and a scientist reported the gap had been already identified by a needs assessment undertaken in 2006 in the ASARECA $^{7}$ region under the CGIAR, which identified the shortage of communication skills and pointed to the need to build capacity in agricultural information communication and management (AICM). Consequently, a postgraduate programme to address the communication gap in research communication has been developed. ASARECA will collaborate with universities in the region to offer the programme starting 2008/09 academic year under University of Nairobi.

These findings match conclusions drawn in the literature. Adewuyi (2008) says that, technological problems by African authors notwithstanding, "research on communicative competence and academic discourse has for long exposed the linguistic problems that African scholars face in writing scholarly articles in the English language”; he adds, "Writing academic papers in a language other than the mother tongue may pose problems” (p. 4). Citing Alo (2003, p. 117), he explains that communication competencies underpin academic research communication success. Both scholars argue that "writers/scholars must be competent in all the components of communicative competence - linguistic, socio-linguistic, strategic, and discourse - in order to produce well-formed utterances and sentences” (Adewuyi 2008, p. 4). Adewuyi goes on to comment that "idiosyncratic use of words and phrases by African authors might impede comprehension of what the author is trying to put across...” (p. 5). A CODATA 2007 workshop by National Research Council, South Africa (CODATA 2007) highlighted scientists’ communication skill gap and recommended for workshops on data handling for they felt "researchers may not be expert in setting up databases to store the data they collect, complete with metadata that is necessary for long term access, and to make it available in a useable format (p. 5).”

\footnotetext{
${ }^{7}$ Association for Strengthening Agricultural Research in Eastern and Central Africa (www.asareca.org)
} 
The communication problem by scientists is global. Gewin (2009) affirms the difficulties in scientists' ability to communicate scientific outputs by quoting Washington, D.C.-based science adviser to president George Bush who argues that, "The quality that is hardest to find in the science policy world is the ability to write clearly and quickly,” and adds that, "Communicating technical material in technical journals does not give you the skills to communicate to nontechnical audiences," (n.p). Gewin (2009) argues that though journal publications may denote one's expertise, they shed " no light on their public-speaking ability - which is necessary to effectively advise policy makers how to adopt sound science”(, n. p). She further cites Cheryl Schaffer, director of finance and administration at the Washington, D.C.-based Union of Concerned Scientists (UCS), who holds that, “A person's science background is a huge asset, but to succeed in advocacy - the ability to communicate a passionate belief in the value of sound science - requires a creative communicator”(Gewin 2009, n. p).

From the discussion above, it is evident that communication skills are critical if there is going to be leverage in ICT-enabled scientific communication to inform Kenyan policy and decision-making and contribute to the development process as per ERS 2003-2007 and Vision 2030.

\subsubsection{Lack of assessment of researchers' needs and institutional e-readiness}

In addition to human capital deficiencies, participants reported the need for ICT adoption for research communication to be predicated on researchers' needs and the research institutions' preparedness, to avoid haphazard implementation of ICT programmes. A research communications' advisor reported that, owing to world pressure to adopt ICT, "the easier thing has been to buy computers; sometimes not even based on real needs" (GO1_Ma), what some participants described as "putting the cart before the horse," or "the egg and chicken issue puzzle” as an ICT lecturer put it. A senior scientist explained implementing ICT without e-readiness assessment has tended to promote ICT technology too fast for the majority to adapt their cultural ways in order to cope. This seems to confirm Rogers' (1993; Rogers 2003) theory that

any new innovation like ICT requires time to be able to reach the threshold exposure for adoption. There was need to prepare the ground for ICT adoption by analysing the 
needs of the target group to lessen social inertia and enhance adoption as some respondents indicated:

These days the world is under pressure to have ICT and the observation I'm making ... it is good to have them but it is clearly not preceded by detailed diagnostics and, therefore, you quickly run into problems (GO1_Ma)

Now it is my duty to conduct almost a needs assessment that would effectively identify ICT needs for the teaching and research community. We have not done it ... (LR2_A).

The call for an e-readiness assessment to establish "the degree to which a society or community is prepared to participate in the global information society" (NdedeAmadi 2006, p. 117) is global and featured widely in the World Conference on Agricultural Information and IT held in Tokyo from 24-27 August 2008. One key point emerging from the sessions was that ICT is about people and processes, not technology and so, “considering that ICTs are [an] enabling tool, we need make sure it is needs-driven” (Onu 2008). The same call was made by Ondari-Okemwa (2002); Duque et al. (2005); Musa, et al. (2005); Ndede-Amadi (2006) and Keengwe, et al. (2009). Ndede-Amadi (2006) argues that "the first step in any approach to introducing ICTs into a society is to consider its ability to integrate ICTs. E-readiness assessment provides baseline information for planning and for making a comparison across countries and regions” (p. 118). Hence, it is important to base any ICT-mediated research information communication system on the researchers' needs and institutional e-readiness.

\subsubsection{ICT tools and infrastructure}

As well as researchers' needs and institutional e-readiness assessment, the findings revealed structural problems that, according to a communications official, made the environment in public research institutions "not technologically mature" for ICT adoption. These included infrastructural problems like low penetration of electricity and telephony, upon which ICT adoption is predicated, especially in rural areas. Where there was electricity, the supply was unreliable, making it difficult to use the computer-mediated communication, as some participants explained:

...the conditions really, particularly ... infrastructure - electricity; the low penetration of electricity in a number of places has made it impossible to adopt things that are electrically controlled. So you can imagine if the problems we have in Nairobi are compounded in the more rural universities ... quite a bit (RS1_G) 
Or if you actually happen to go to the village you may find that you don't need actually to carry your laptop because there is no power. So those are some of the things that structurally, socially, culturally and economically may not actually be having a lot of advantages on us purely because of the way the country is (LR1_A)

... We don't have adequate electricity to enable people to use computers well. We don't have adequate telecommunications facilities to enable us to use the Internet well (LR2_A).

This affirms findings of earlier studies in Kenya and Africa (Bowman-Ngaruiya and Waema 2006; Government of Kenya 2006; Wafula and Etta 2006; Bagchi and Udo 2007; Casal 2007; Government of Kenya 2007).

A senior lecturer cum ICT director in university B attributed the lack of ICT tools and infrastructure in research institutions to the failure by relevant ministries to address the issue:

There is a Ministry of ICT which is very busy actually trying to work on infrastructure outside. So we need their help also to help us address our infrastructure inside. So far they are not doing that at all... the Ministry of Energy needs to make sure that we have constant supply of energy within these institutions. It is very frustrating really. We get [a] power blackout every single day and it really causes frustrations... (LR3_B).

A library manager from international institute E reaffirmed the lack of ICT tools and infrastructure in public institutions that would facilitate access to e-resources. She explained there were efforts by international e-resources networks like PERI, HINARI and AGORA to make available research published in high impact journals but noted that access was still very limited due to small bandwidth, lack of facilities, infrastructure, computers and reliable Internet. She mentioned E was assisting researchers in public institutions to access e-resources through their information centre.

A senior scientist cum editor reported that lack of Internet wiring/cabling in buildings, new and old alike, including the donor-funded structures, was a problem affecting ICT adoption in national institutions. He explained there was need for government building planners and donor-funded project managers to factor in Internet cabling from the beginning, just like water and electricity, as installing post-construction cabling and wiring was expensive. This was a major barrier to ICT adoption, because many government institutions first had to provide funds for cabling before they could think of Internet connectivity. He said this contrasted with the culture of the Western/developed world where Internet and telephone wiring is part of the construction contracts. 
Notwithstanding the fact that Japan is a high power distance developed country, the findings above could be reflective of the difference in societies like Kenya where relationships are vertical, resulting in controlled communication and less emphasis on communication infrastructure. This is unlike low power distance societies where relationships are flat and thereby communication is seen as key and valued (Hofstede 2001); hence, care is taken to include communication infrastructure within the building process. The situation could also be an indication of the intricate relationships in donor-funded projects, which in itself is another form of power distance.

Participants reported lack of infrastructure has led to disparities in access to research information, creating a scenario where, as a senior scientist explained, "it is the people who already have the information who have access but the people the information is intended for do not have access” (RS1_G). Internet access was lacking at grassroots where research findings are implemented. This translated into the Internet being a tool for the educated/the elite in the advantaged urban areas. Participants expressed the need for functional information communication systems to facilitate dual communication between those farmers/others at the grassroots needing information and the researchers. The two sides needed to be provided with modern tools for communication, in order to facilitate information exchange. This way, dissemination of technologies and/or methodologies would be more likely to succeed and have the desired impacts, as a senior scientist indicated:

... whoever you are communicating to should be able to access the tools of the trade... how many agricultural communities or the end-users of agricultural technologies have access to Internet? ... So what are the information nodes at the grassroots level do not exist. A farmer has no access ... So if it is one way, it is unlikely to have the kind of impact we need because a researcher needs to be communicated to: Is your research finding/ are your methodologies working? ... (RS5_E).

A senior scientist suggested providing farmers with modern communication tools like digital cameras, or establishing information points at grassroots where such tools could be accessed. This would enable farmers to immediately share their problems online and thus open up the issue for advice from all over. It would also be more effective than the conventional scientific communication channels of sending samples to the laboratory for analysis before assistance can be given: 
Let me give you a scenario. Supposing a farmer, a horticulturist has 200 acres and he observes that there is a problem with his crop, it would be easier if he had just a digital camera or a local information centre could take a photograph of what the problem is plus narratives from this farmer, relay it to the Internet and let it reach as many professionals as possible who could interpret this quickly and relay back what they think the problem is and what are the likely solutions. That farmer may not lose as much as when somebody tells him you take a piece of this, go to a lab and the lab may not have the materials to analyse this and in a week the crop is gone if it was a bacterial or a fungal problem, for example. The crop is gone... (RS5_E).

A library manager also suggested establishing information kiosks as possible inlets and outlets for input and feedback, as a means to minimise the disparities in infrastructure (see section 7.1.1.2 above).

The findings revealed that disparities in access to ICT tools and infrastructure also existed within and between research institutions. There was good Internet presence in international research institutions. However, public institutions, apart from those that enjoyed donor funding, often lacked Internet connectivity or adequate PCs, which in some institutions were initially confined to one place. As scholars and researchers explained, the lack of tools tended to discourage many from adopting ICT for research communication, especially those in rural areas, which could be a possible explanation for the initial slow penetration of the ICT-mediated communication in Kenya:

... we have no computers in our research centres connected to the Internet - connectivitywhere researchers can be able to access information in terms of scientific knowledge, what others are doing ... we don't have that connectivity. It is a limiting factor to our scientists especially the ones outside Nairobi. Here at $\mathrm{C}$ headquarters we are connected (RS6_C)

There are still cases where you find that a pool of lecturers is using may be a few PCs to work and in such cases it is not easy. Actually they opt not to use it because they don't want to keep on queuing for one facility. So they tend to go back to the traditional methods (LR3_B).

Where there was Internet connectivity, researchers reported that the speed was very slow and, therefore, it was difficult to access information. Unless they were lucky enough to also have part-time jobs in the private institutions, researchers had to go to cyber cafes, which was too expensive to be affordable for research communication purposes.

Some participants reported the lack of Internet connectivity in most national institutions meant that those who did have Internet access were unable to communicate their research outputs to their peers: 
... in as far as ICT is concerned, $\mathrm{C}$ is among the most advanced institutions in Kenya. If I gauge myself with people who are working in other ministries apart from research institutions, they don't have ICT. ... You see my computer here; I read my emails every day but somebody in University A has to go to a cyber café. ... Other institutions are behind us and because we are not at par we cannot be able to communicate (RS6_C)

... internally here I can send a document of whichever magnitude but if I send the same document to somebody outside they will have problems downloading that information. The issue is the recipients: is their ICT to such a level that it can be able to receive those bulk documents? ... if you send that to somebody through yahoo it might never open. It might not even go. So it depends on whether the recipients have the capacity ... Although we are in digital wireless from ICP I think the other systems most of it is still analog (GO3_F).

It emerged from the data that inadequate bandwidth in many public institutions was also a barrier to ICT-mediated research communication, as two lecturers and a scientist explained:

In terms of bandwidth ... we have $1 \mathrm{MB}$ going out and $4 \mathrm{MB}$ coming in. A university this big would require bandwidth in terms of gigabits, not $4 \mathrm{MB}$, or it should be like those universities in the West that are operating on the Internet ... (LR2_A)

Like in this university you find that about half of the staff use emails which are foreign like yahoo, like hotmail... because if you go to yahoo they would give you space of about sometimes $1 \mathrm{G}, \ldots$ if you come to ours we will give you maximum of 20MB because of course we can't afford to give you all that space (LR3_B)

... I'm told you cannot go beyond ... $16 \mathrm{MB}$... So we hit a snag when it comes to that kind of restriction (RS2_D).

This made downloading and uploading of information quite difficult. It was hoped the new East African marine systems cable, a fibre-optic undersea cable that was being laid, would change things and extend ICT use even in schools, as one scientist suggested:

... this new fibre cable they are trying to initiate in the country. When that is done there is a feeling that we are going to have a lot of things changing... (RS2_D).

[This was launched on 12 June 2009 in Mombasa ( Daily Nation 12 June, 2009).

On the whole, there was general recognition of the importance of ICT in scientific communication and a move towards electronic formats. However, although print would still be important until the environment fully supported electronic communication, as a library manager and a scientist observed:

The way I see it [research communication] changing is of course it will go electronic. But I still think even the print is very important until the kind of environment we operate in is also fully electronic (L1_A) 
Let me say that ICT today is the technology that can be able to make our scientific outputs to reach the farmers. By using ICT we would be having effective and efficient research due to the fact that we have limited resources in terms of personnel, infrastructure- vehicles, laboratories, computers... (RS6_C).

Thus, despite the challenges of the current work culture characterised by busy schedules, a senior scientist noted a changing research culture, influenced by the ICT forced one to be requirements to be up-to-date in your field. He felt this was promoting ICT adoption, despite the limited ISPs:

Today you'd be accused by the international peers if you didn't know what has been reported may be last year or may be a few months ago... (RS4_E).

The findings above show that less-than-adequate tools and infrastructure are a great barrier to ICT-enabled research communication, which points to the need to address the problem.

\subsubsection{Appropriate content}

In addition to tools and infrastructure, lack of appropriate content emerged as a key barrier to adoption of ICT for research communication in Kenya. All public research institutions visited during the study had websites, but these tended to be characterised by scanty or irrelevant research content, rather than effectively communicating research outputs. Quite a number of public institutions' websites displayed information on departments, institutional vision, mission and mandates, and profiles of the administrators - what one participant referred to as the "African disease - the big man disease in Africa” (GO1_Ma). Some respondents commented that most websites were still under construction, were not interactive and provided no links, as the following excerpt shows:

... at best sometimes you just had the name of the VC and a few others. In other words, they were not communicating any information ... I suspect it is the main reason why African universities have really featured badly in Internet-based surveys of the ratings of the universities because there was no information relating to their work (LR3_B).

The lack of Internet local content supports findings from earlier research on public sector ICT research initiatives in Kenya. Ndede-Amadi (2006) found that many people were unclear about the content that should be placed on web sites while Wafula and Etta (2006) called for development of e-content to serve Kenya's diverse cultures. Oyomno (2006) reinforced the fact that content and applications are important aspects of a national ICT environment. 
That the "big man syndrome" was affecting the content that goes into public institutions websites aligns with studies on the influence of culture on ICT adoption, especially in developing countries like Kenya. High power distance tends to make those in power suppress development of a new innovation like ICT (Goodman 1991; Bagchi 2001; Hofstede 2001; Bagchi and Udo 2007), resulting in slow adoption and use.

Where web content was available, participants reported that was not well organised and its nature and format were unsuitable for web hosting. As a library manager and two researchers pointed out, information needed to be reorganised, digitised and repackaged into formats that could be availed online for greater access:

... and the person who has the content, the content is not organised well enough to go on to the website. So you find there is a gap between the infrastructure and the content, Florence, and to me it is a very sad situation, very sad situation (L1_A)

... we do not have actually structured information of actually putting the information in the media as a university so that actually the community can go and gauge for themselves what is happening (LR1_A)

... there is a need to be more active in repackaging our reports and also repackaging them in a way that we can host on our website... for greater access... (RS4_E).

As discussed in the previous chapter, participants explained that lack of content could be due to the inappropriate focus on acquisition of the ICT tool and employing technical ICT staff, without thought to the content they were meant to communicate, as well as unclear understanding of ICT potentiality and use in research communication (see 6.1.1 and 6.1.2). In addition, researchers reported they lacked time to generate research outputs that could be disseminated, let alone to publish on the Internet (see 7.1.2.6). Moreover, a scientist pointed out that few of the journals they published in were Internet oriented.

A renowned scientist from an international institute attributed the lack of web content in institutional websites to the digital divide between the poorly endowed researchers in Kenya and rest of Africa and the well endowed developed world. He maintained that researchers in Africa have been separated from the rest of the world owing to inadequate research funding. They could not afford to subscribe to the up-to-date research literature usually published in journals in developed world in the West and, therefore did not have access to research findings. Internet was, therefore, seen as a 
means to address scientific isolation, providing an opportunity to catch up with the rest of the world in that African researchers could access research materials online: "[researchers from Africa] are really at the moment much less feeding into it [Internet] as getting out of it” (RS1_G). The scientist explained researchers were still catching up and trying to familiarise themselves with ICT-mediated communication.

That content is vital in any research communication infrastructure cannot be overemphasised. The content must also respond to the needs of the research community, in order to entice researchers to adopt a computer-mediated research communication system, not only to download knowledge, but also to upload their research outputs for others to share. The findings affirm Borgman's (2007) proposed layered cyberinfrastructure model, in which the supremacy of content is on the top while the ICT infrastructure comes at the bottom. She stresses that "To be useful, the content layer must include the right resources, tools, services, and policies for the communities it serves,” (p. 186).

The challenge of populating the websites with the right digital content is significant in Kenya. Limo (2008) explains it thus, “Anyone keenly listening to technology will appreciate that Kenya's spirited quest for fibre-optics communications is essentially a shift of the focus from computing to communication... But the real driving force for business, regardless of the mediating technology, is the provision of compelling content” (n.p).

Digital content must address institutional needs and goals, right structure, functionality and pedagogical/research principles. As Limo (2008) argues about digital learning content, "the idea is not to have some mesmerising multi-media presentations that have little to achieve in education. It must address a learning need" and "its structure and functionality must fit the curriculum set by the authorities concerned. It must also adhere to pedagogical principles like being student-centred in approach” (n.p).

From the discussion above, it could, therefore, be concluded that any contextual framework for ICT-mediated research communication should include appropriate content. 


\subsubsection{Demand for research/teaching management information systems (MIS)}

In addition to lack of appropriate content, the data revealed that there seemed to be no demand for ICT-mediated research communication systems. An ICT manager cum lecturer in university A reported that, while the administrative and financial departments within the research institutions, especially universities, pushed to have relevant MIS, the research fraternity did not express a need for either teaching or research software:

... the other functions-teaching, learning and research might have not already adopted or created a very strong need for the systems ... to be geared towards them... we have been busy trying to get the VC all sorts of management information systems that can assist him to run the university ... Funny enough there hasn't been a demand by lecturers (LR2_A).

Hence, many research institutions "have all these management information systems and none of them is geared towards specifically research, teaching or consultancy (core functions),” added the ICT manager cum lecturer.

The lack of demand was also evident in the non-use of the existing bandwidth for research communication, as reported by scientists cum science editors:

... how we can be innovative in translating that access into accessing the right information because I can see a lot of misuse of the same technology where people are accessing information that may not be as useful to them (RS4_E)

... the percentage of Internet related access that we have established where people actually use our Internet for research activities is very small. In fact it is probably in single digits, if you say $1-100$. We are not even using more than $10 \%$. So people go to Internet to open The Daily Nation [newspaper] the whole day to read. ... People just go to check and write emails to their friends... (RS5_D).

The ICT manager cum lecturer above said that the lack of demand for research information management systems was reflected even in ICT training, which focused on basic office automation, email and Internet. As a result, “there hasn't been any prepared plan towards some research software except where there are some research elements covered by the presence of Internet.” This highlights the need for research institutions to come up with innovative ways of exploiting the benefits of the Internet, for improved communication of research outputs.

The findings are also supported by the literature. Existence of IS, or MIS, is one level of an ICT application's functional capability, which provides information to meet internal operational and management needs of an organisation and defines a 
conventional computing environment (Oyomno, 2006). The lack of research and teaching MIS signified a lack of functional capability to engage in ICT-enabled research communication for most research institutions. It could be one of the reasons for the reported lack of online presence for most research institutions. There were no web-based applications to provide information that would meet internal and external stakeholders' needs. Participants reported that hardly any downloadable information was posted on the research institutions' websites.

On the other hand, there was a dilemma for the ICT department, as reported by the ICT manager cum lecturer above. He explained that, although there seemed to be no demand for Internet, whenever they increased the bandwidth it was used up immediately. He commented this indicated implicit demand for Internet within the research community and said informal talk confirmed the same.

The dilemma concerning implicit demand for Internet could stem from the lack of needs assessment to establish researchers' needs and priorities (see chapter 5 and 7.1.2.4). On the other hand, the lack of demand for research information system resonates with the lack of a research culture discussed in chapter 5 (see 5.2 .1 and 5.2.3.3), where seeking information was not a priority for many. As such, many did not resort to Internet for research information (see 5.2.3.3).

\subsubsection{Resources}

Further to the barriers related to demand for research MIS, the findings revealed funding and time to be inhibiting ICT-mediated research communication.

Regarding funding, participants expressed that there was no or inadequate funding specifically allocated for implementation and sustainability of ICT infrastructure and programmes. As discussed earlier (see chapter 6.1.1), lack of appreciation of ICT had led to lack of ICT prioritisation nationally and institutionally. Consequently, ICT was not a budget line for most institutions, as some participants reported:

Our budgets are usually low and ICT infrastructure, particularly the initial cost, is very high so you find you cannot really get what you want on the institutional budget ... the funding problem is a reality but a reality which could be handled (L1_A)

So money is a threat to acquiring the kind of technology we want for this country in our research institutions. The government has to think twice about how to allocate funds to 
facilitate acquisition of what is considered to be the best tools to facilitate research ... (RS2_D)

... the Ministry of Education needs to help us in addressing ICT so that it can have allocations to help the ICT needs, right now they are not doing that (LR3_B).

Participants explained that most ICT programmes in public research institutions were donor-funded and there was no associated sustainability component, despite high initial and subsequent maintenance costs:

... then of course if you did get one [ICT] from a donor, sustainability is zero- after 2, 3 or 5 years it is down the drain (LR3_B)

... there a few projects in some centres where people have taken a little money from their projects where when you writing your proposals you talk of emails. I hear you can say you spent around a hundred thousand Kenya shillings (Ksh.100,000). They have pooled those resources - one scientist has given Ksh.100,000, another one Ksh. 100,000 and they have bought a line. But when the project is over, the line is [disconnected] (RS6_C).

This affirms earlier ICT studies in research institutions in Kenya and Africa (Rosenberg 1998; Mutula 2001; Ondari-Okemwa 2002; Oyelaran-Oyeyinka and Adeya 2002; Rosenberg 2005; Odero-Musakali and Mutula 2007).

As a result of lack of ICT budgets, a research communications advisor reported research institutions and their outputs "are not visible in the Internet.... In fact you see that continually our universities are rated low because the Internet has become so important in making research visible” (GO1_Ma). There was a need, therefore, for government to rethink and prioritise ICT in budget allocations, as a first step towards ICT-mediated for research communication.

It also emerged from the data that many public institutions lacked funding for subscriptions to online sources of research information, such as journals. The library manager in university A reported that libraries might not be able to guarantee access to current information that the research community needed to identify research gaps. In addition, they did not have the ICT facilities to enable them to easily access research information, even where donor organisations had made provisions for such materials.

Regarding the possibility of publishing in open access journals as a means of overcoming the funding limitation, a library manager indicated this might be 
challenging because some required author fees (a model of publishing where an author pays for the cost of publication). These are not cheap for most scientists:

But you see now, from my little knowledge of open access journals, they may sound free but there is an aspect of paying. In some cases they are charging what they call the author's fees and the author's fees may be quite high, almost the same as the subscription fee. ...I would think that may affect the authors because if the authors would have to pay those authors' fees and anything that increases the cost of what they were doing before I'm sure it would not be encouraging them to publish (L1_E).

Again, there is the issue of impact factor. A library manager indicated that many scientists would like to publish in an open access journal if it is rated highly:

You are talking about the open access journals. For example, there is what is called Nucleic Acid Journal, which I can remember because we were buying it and then it went on open access. Our researchers used to/continue publishing in it as an open access journal because I think it is still rated the way it was rated... (L2_E).

Participants emphasised the necessity to have open access journals which employ the no fee model for both access and dissemination, first because research is publicly funded and, therefore, a public good, and secondly because the majority of the scientists from Kenya and Africa in general could not afford subscriptions. Thus, unlimited open access was viewed as having considerable potential to overcome the research communication problem in Kenya. Researchers also called for sound Internet connections, if they were to resort to the Internet as an information source and means of disseminating research outputs.

The poor funding and facilitation in public research institutions contrasted sharply with the well financed international institutions and donor funded public ones, as a library manager from an international institute and a senior scientist from a national one reported:

... in E we have extended our services to the universities that are dealing with agriculture and we allow them access into our information centre free of charge including the Internet café where they should be able to access the online journals that we subscribe to or any other free journals that are available on the Internet... (L2_E)

First let's appreciate that actually there are a lot of facilities outside there which we cannot easily access. One of them is funds. The institutions out there are well funded... it is either you have money to buy your computer or you wait until the university gets enough money to buy you a computer. By the time actually the university buys all these computers it is going to be sometime within a number of years (LR3_B).

However, some participants disagreed with the claim of lack of funding and research resources. They attributed the slow adoption of ICT nationally and institutionally to a lack of professionalism in government when it came to planning and investing time to 
ensure the plans were appropriately executed (section 7.1.1.3). Mutula (2001; 2001) and Odero-Musakali and Mutula (2007), in their studies of ICT adoption and use in university libraries in East Africa, Kenya in particular, seem to concur with this claim, accusing libraries of passivity in their efforts to adopt ICT. Citing examples from the southern African region who have become ISPs, Mutula alleges "universities in Kenya continue using "limitation of funds" as an excuse to their non-active participation in these technological developments” (Odero-Musakali and Mutula 2007, p. 472).

In addition to funding, researchers reported a lack of time, not only to publish on the Internet research but also to undertake research that could lead to outputs to be disseminated (see also 7.1.5). One requires a lot of time on the computer to come up with good content. However, researchers, especially those who teach, do not have much spare time, owing to large numbers of students. Moreover, their ICT skills might be inadequate to allow fast Internet surfing, as a lecturer indicated:

... time because of the persons in numbers and of the skills in using actually the ICT materials you find you have to spend a lot of time and this may not actually go well especially if you are strategist teaching. You may find now research is going to suffer so that you can be able to go and teach (LR3_A).

I don't think they have the time to write into the Internet ... (RS2_D).

The time factor contributing to a lack of online content align with findings from studies on adoption of ICT by faculty members (Hebert 2007; Keengwe, et al. 2009). Keengwe et al. (2009) reported,

"Nearly all respondents indicated that technology adoption was a time consuming process. With pressure to teach well, conduct research, service commitments, accreditation assessments, professional development, and meetings, the faculty reported that they lacked time for additional responsibilities” (p. 26).

The findings and the discussion above show lack of or inadequate resources were a major hindrance to ICT-mediated research communication. The situation, therefore, calls for concerted effort to address the resources gap.

\subsubsection{Motivation}

"A scholarly information infrastructure will be effective in facilitating access to the artefacts of research if it takes into account the motivations of those who produce and control those artefacts” (Borgman 2007p. 177) 
Further to resourcing issues, lack of motivation was reported as a factor affecting ICT-mediated research communication. Earlier on in chapter 5, motivation was identified as one of the needs to reinforce strategic research communication in Kenya (see 5.5). It also emerged that it was a barrier to ICT-enabled research communication.

The reported hard economic realities and poor remuneration and terms of service for public servants, including scholars and researchers in the public institutions, had forced many Kenyans to look for alternative ways to earn their livelihoods. Participants reported that the economic hardships had resulted in a new culture popularly known as "What's in it for me?” where Kenyans pegged their adoption and use of any innovation on its potential for financial gain. A research communications official explained it thus,

...in terms of national values we [Kenyans] are very aggressive financially. People seem to be very money-minded. If the computer is a source of money people will invest in it (GO1_Ma).

Hence, financial incentives were determinant in ICT adoption in Kenya. One participant reported that many cyber cafes sprung up in Nairobi and other cities and towns when people discovered they could make money that way. This view was supported by a senior scientist who argued that a Kenyan will not "spend two hours on the Internet, unless he knows it has a benefit to him and unless that Internet is free" and even then "we [Kenyans] limit our scope to what information we want to get" (RS3_F). Such a culture is not healthy for research communication, which requires extensive exploration of the literature to identify gaps. A researcher expounded on this when he said they had to make passing a computer course in health management mandatory in order for students to adopt ICT.

Thus, commercial and demand driven incentives were required to force adoption of ICT, owing to the poor motivation of the research community. According to some participants, there had not been wide application of ICT to research communication, because it was not perceived as having immediate financial benefits. Conversely, participants reported high adoption of ICT by the business community.This culture of pegging participation in ICT-mediated research communication on monetary benefits is an indication that, in developing countries like Kenya, the issue of meeting basic needs overrides the struggle for higher professional status. This contrasts with research practice in the developed world where researchers have gone past the basic 
needs baseline to the level of working towards higher professional status predicated on one's research outputs; thus, confirming the general parallel trend in research between the two worlds as revealed in the literature (DiMaggio, Hargittai et al. 2001; Kaniki 2004; Gebremichael and Jackson 2006; Casal 2007).

Further, the Internet is expensive and researchers' salaries too low to afford to pay for online searches for clients; hence, they visit Internet cafes only to send emails and other personal use. One scientist summarised it thus:

... I am a scientist but when I am looking for information for other people I cannot go looking for it in the cyber cafes. I only go there to send emails and what have you. You know it is also a cost and our salaries are very little ... Rarely will you see a person going to search for information for the farmers ... (RS6_C).

This confirms Borgman’s (2007) claim that "a scholarly information infrastructure will be effective in facilitating access to the artefacts of research if it takes into account the motivations of those who produce and control those artefacts" (p. 177). Rogers, Takegami and Yin (2001) call for adequate resources and organisational and other reward/incentive structures to fast-track the difficult process of technology transfer. Thus, researchers' motivation is vital for ICT-mediated research communication.

\subsection{Chapter Summary}

This chapter has addressed factors related to both the external and internal organisational environments in Kenyan research communities to determine how they affected ICT-mediated research communication. The findings have revealed a number of issues that need to be addressed to allow the diffusion and use of ICT for research communication purposes. First, the lack of enabling government and institutional ICT policies, implementation strategies, legislatory and regulatory environment has resulted in ICT not being integrated into daily work routines. It also emerged that organisations often lacked capacity for ICT-mediated research communication and so there is need for them to build capacity in the following areas:

- ICT awareness and skills to equip researchers with the requisite skills and functional capability to engage in ICT-mediated research communication;

- Communication skills to enable researchers to originate outputs and in repackage it formats that can be disseminated online; 
- researchers' needs and institutional e-readiness assessment so that ICT adoption and use is pegged on what researchers and organisations need to realise their vision and strategic plans;

- ICT tools and infrastructure upon which ICT-mediated research communication is predicated; and

- Development of appropriate local content that answers the people's socio-cultural needs.

Moreover, there was a lack of demand for research and teaching management information system(s) owing to lack of functional ICT capability, resulting in no push to adopt and use ICT for research communication from within the research community. Lack of or inadequate resources in terms of funding and time were also a great barrier, in addition to researchers' poor motivation. Table 7.1 gives a summary of the field findings on the organisational factors.

Table 7.1: Summary of Institutional Factors

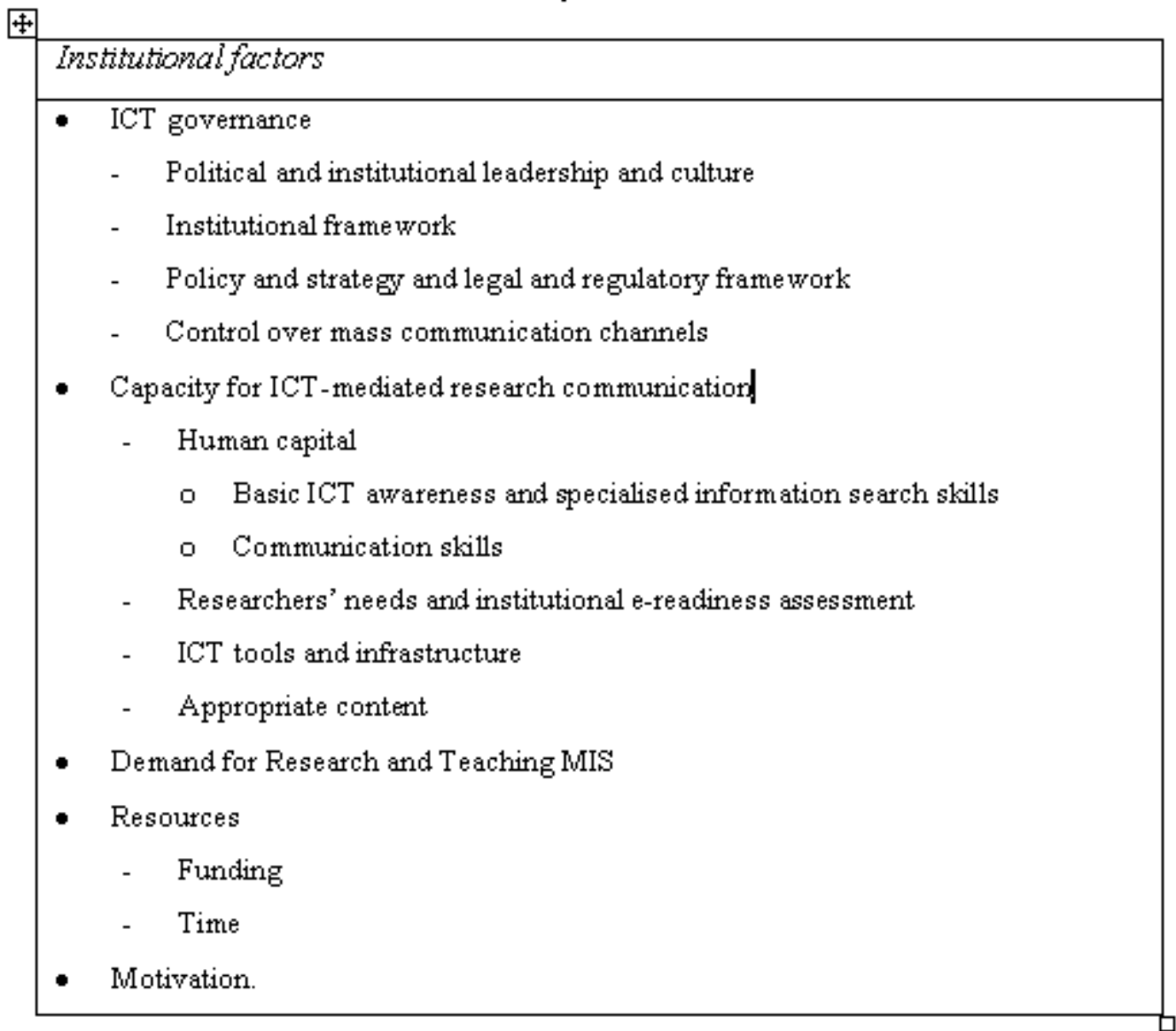


Generally, this chapter's findings on the influence of the institutional environment affirm the diffusion theories by Rogers (2003) and Hofstede (2001). The findings specifically share Rogers' (2003) view that the types of innovation decision (governance issues) and available communication channels to create awareness about the innovation affect its adoption.

Nevertheless, the study revealed institutional features unique to Kenyan situation, such as political and institutional leadership and culture; institutional framework; policy and strategy and regulatory framework and issues to do with control over mass communication channels. The study also unveiled factors that are critical to the adoption and use of ICT, which the theories did not emphasise. These included capacity building issues such as skilled human capital, needs and e-readiness assessment, ICT tools and infrastructure and appropriate content. Other issues that arose included demand for research and teaching MIS and for resources in terms of funding and time. These aspects seemed determinant in the adoption and use of ICT for research communication in the Kenyan context; thus, necessitating the revision of the model to reflect the Kenyan situation (see figure 7.1).

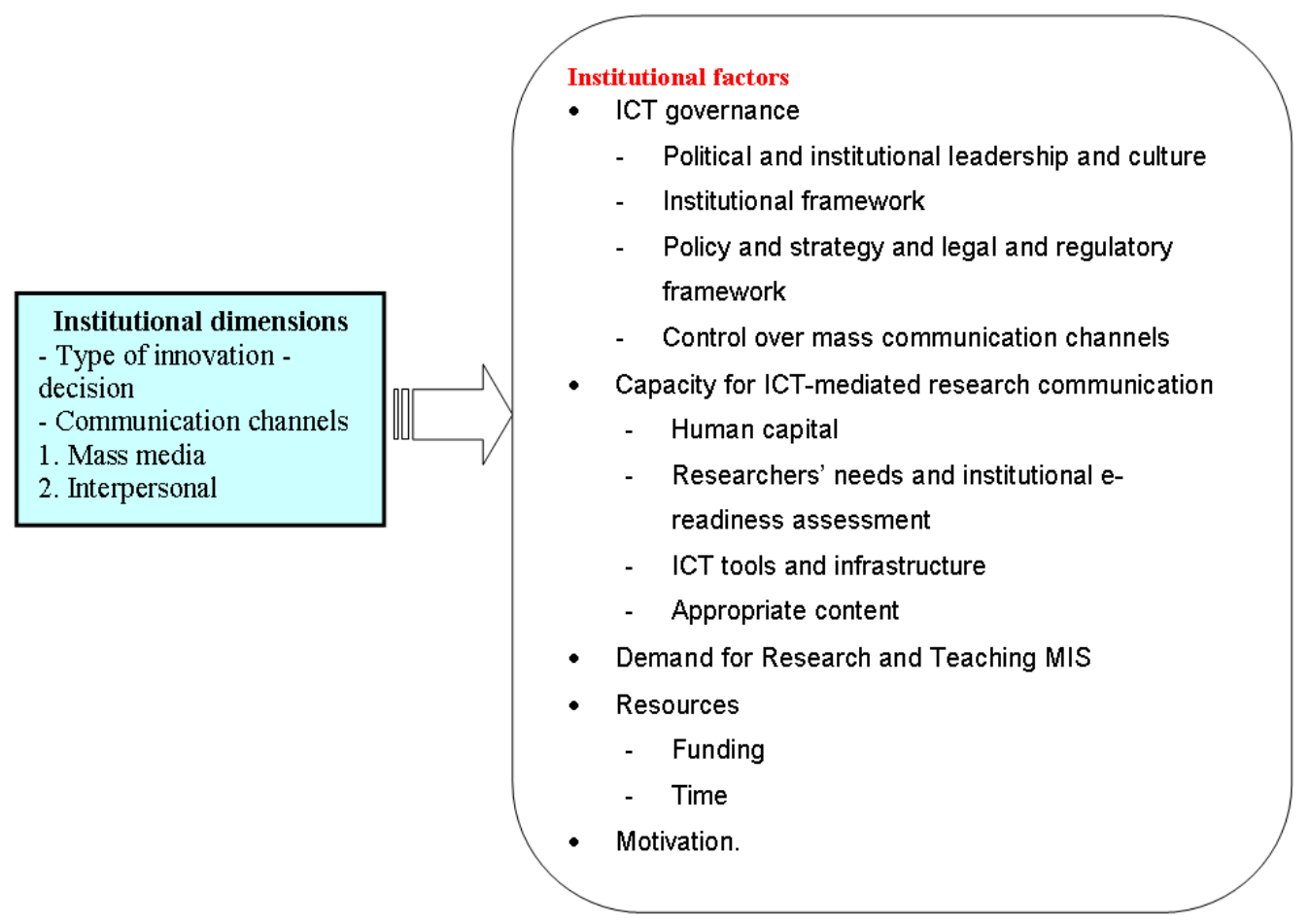

Figure 7.1: Revision of the institutional dimensions 
Addressing the above institutional factors is of importance if an ICT-enabled research communication culture within the research community is going to develop, which could ultimately help institutions disseminate their research outputs and contribute to achieving their goals and the desired impacts. It is only then that ICT could contribute towards informed agricultural, environmental and health practices which could ultimately improve people's livelihoods as per the Kenya Vision 2030 (Government of Kenya 2007) goals and objectives and also the 8 UN Millennium Development Goals of alleviating poverty and hunger, health related issues, through to developing global partnership for development (www.un.org/millenniumgoals/goals.html).

This chapter leads to the last chapter on presentation and discussion of findings - the technological environment and other dimensions in ICT-mediated research communication - a continuation of the answer to the third and fourth research questions as outlined in the introduction of chapter 6. 


\section{CHAPTER EIGHT}

\section{TECHNOLOGICAL AND OTHER FACTORS}

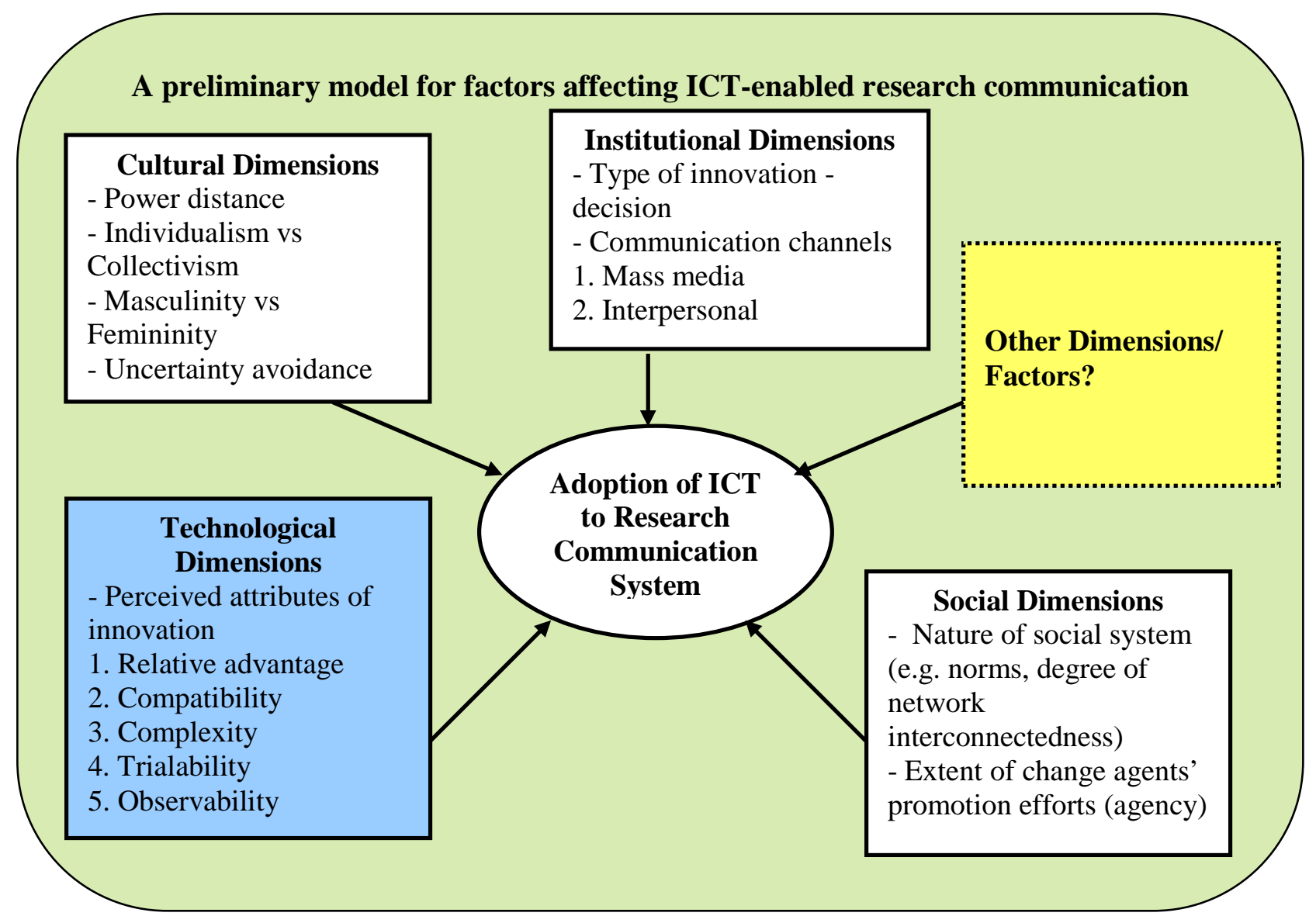

\subsection{Chapter Overview}

The previous chapter analysed the factors in the institutional environment that affect the adoption of ICT for research communication in Kenya. This chapter addresses issues in ICT adoption that are related to the technology itself from the point of view of those who had access to ICT and also presents other factors.

\subsection{The Technological Dimension}

Though some participants regarded ICT as "a technology that kind of confuses many people including even the educated” (RS5_D), there was recognition that ICT awareness and use were increasing. A government official indicated that computers were "not any longer miracles" (GO2_Mb) and that "acquisition of computers these days is an ordinary thing." The interviewees reported a variety of technology-related factors centering on ICT's perceived attributes, which affected ICT-mediated research 
communication in Kenya as presented in the subsequent sections. The diffusion literature reviewed earlier showed that perceived attributes of any new innovation, such as relative advantage, compatibility, trialability, reliability and observability, affected the rate of adoption (Bagchi \& Udo, 2007; Keengwe, et al., 2009; Nystrom, Ramamurthy \& Wilson, 2002; Rogers, 1995,2003; Schneider, 2007; Schrum, Skeele \& Grant, 2002; Tetiwat, 2003; Tornatzky \& Klein, 1982; Tran, 2006). The findings of this study indicated how these attributes have enabled and challenged adoption of ICT for research communication by research institutions in Kenya.

\subsubsection{Relative advantage}

All the interviewees who had access to ICT considered that ICT had a relative advantage over other technologies and felt that its adoption was mandatory for proper functioning in scientific research, be it publishing or searching for information, as this quote from a lecturer indicates:

We can't complain about the relative advantage. It has all the relative advantage (LR1_A).

A scientist commented that computer-mediated communication was the way to go "not a thing to gamble about. It is something of very high priority in any institution or country that wants to go doing research” (RS2_D). Other participants explained ICT had brought efficiency and effectiveness to the research process by providing fast and convenient access to information to both researchers and clients. They said researchers could now easily access varied current information - papers, abstracts and reports - from anywhere in the world "simply by googling” (RS3_F) unlike before where researchers had to travel long distances to look for information from libraries at the headquarters:

ICT facilitates our research, creates an enabling environment where we can be able to access whatever information we want from all over the world. This makes our research to be effective and efficient. Effective in the sense that we are not using so many resources - are not expected to travel to look for information let's say in town or another centre or scientific institution. I just come here and punch the machine and it is there ... Even for some of our stakeholders and partners it is changing them because they can be able to access information within a very short time. Like before the Internet came, if I wanted to do some literature search sometimes I would fuel a car all the way to Nairobi to visit the library either at University A or C headquarters. But now if a far off centre is connected to the Internet, the scientists and our learned partners would get the information they required from the net (RS6_C).

If they take advantage of it, it makes the work much, much easier, more effective and higher quality because with ICTs the world has become small. So as a researcher through ICTs you have everything within that laptop... (L1_A). 
A senior scientist added that the fast accessibility to research information through ICT "improves your thinking” (RS3_F) and so served to strengthen scientific communication and research depth through timely identification of gaps and methodologies “that can sharpen your own ways of doing research” (RS4_E).

Participants reported that the availability of ICT has increased scientific productivity, which would keep increasing as more young people became exposed to and use ICT for scientific communication. They said having ICT had made researchers' work much easier, more effective, and of higher quality. Some gave examples of how it alleviated the difficulties of preparing and communicating research outputs through the cumbersome traditional methods of manual report writing and snail mail. They indicated their reliance on traditional methods of research communication had led to the apparent lack of scientific output. Now, such researchers could process reports and organise events through email and Internet in record time:

I can guess here and say if somebody compared whatever is being produced now with the scientific work that was produced 20 years ago when computers were not easily affordable there would be a big difference. Scientists are producing more, even with the difficulties of old ones using new technologies... that is something observable at the moment and I can foresee this changing greatly in let's say 10 years to come when a lot of our ICT users, very many of them young, will have grown with the new technology and giving a lot more ... (RS2_D)

You are seeing me organising meetings. Look at this letter which I read from the Internet... The letter is from ASARECA in Entebbe. The person just wrote email yesterday. He is coming to do work in Kenya...It [ICT] facilitates my work because if they were to write a letter, it would have taken two days or a week (RS6_C).

Participants reported that ICT facilitated networking of "those people with similar goals about some areas of research” (RS2_D). It had helped forge researchers' relations, irrespective of distance:

ICT makes our relations closer in the sense that someone I could not see physically it is very easy to put something in the computer and the person is reading in the next one minute. So communication is very fast and it even brings people closer... if I want to talk to somebody in the US or Europe or Australia it is just a matter of seconds. I punch something and send (RS6_C).

ICT had also made it possible to access researchers' profiles online, thus, facilitating communication, as reported by one researcher:

Another thing that I find useful with the Internet is the fact that I can type any scientist mail. If I want to know what the publication track is, I just go to Google and 
see where his name appears. So you get to know a scientist even before you have met them face to face (RS3_F).

Another reported advantage of ICT-mediated research communication is that it made it easier and faster to acquire grants:

I used his computer to apply for grants, just to show you how easy it would be when you have a computer ... I have been here for quite a long time but before I used that computer I hadn't acquired any grants... When you have this new technology you can reach the world within a very short time and get what you want (RS2_D).

Also ICT provided partners/colleagues with immediate backup, as reported a scientist:

But an advantage with Internet is the fact that when you send your documents out... you know it is secure somewhere. So even if your computer crashes you know you have a document somewhere that you can work with if you are working with a partner... (RS3_F).

A unique feature of ICT mentioned by a scientist was its ability to be customised to the needs of institutions, thus maximizing its benefits, as was the case in the developed world:

... computer and internet technology is an instrumentation facility... I have been emphasising to all researchers and directors of research centres ... build your own instrumentation information facility in your department. Design it to fit you rather than saying somebody should do it for you. It is not like water and electricity you find in the labs... (RS5_D).

Participants explained that the Internet impacted positively on the publishing of research. They said it facilitated rapid and cost-effective publishing, as the submission and reviewing processes were done electronically and it allowed scientists to clarify issues during the course of publishing. Thus, as a scientist added, "Internet has taken publishing to another level... has really revolutionalised the way we publish.” Internet, therefore, "is an amazing tool for an African scientist to have access to in terms of publishing science and pushing science to new frontiers” (RS3_F). Through websites and mailing lists or specialist discussion forums, researchers were able to get advice and answers to problems from all over the world. Hence, a research scientist concluded: "whatever limitations there are ... it is the best means of communicating among the scientists because we can do a lot using this within the limits. I can qualify it as a very good technology for scientists” (RS2_D).

Nevertheless, despite the many advantages of ICT, participants reported an inherent fear of computer-mediated technology which needed to be overcome before it could 
fully be embraced, as also discussed earlier (see 6.1.1.2). One of the participants observed:

So it is not even our scientists; this is a technology that people are still inherently afraid of. ... this technology must be integrated into our operational strategies at every level (RS5_D).

As also discussed in chapter 6, interviewees stressed the need to combine computermediated ICT with other tools like radio and the traditional baraza, for these are the tools that are easily accessible and still cherished by farmers and other users at the grassroots. They felt that embracing multi-media ICT would facilitate participation in the research communication process by all and would also address cultural aspects, like orality of a community (see 6.1.6.1).

Another issue, as a scientist observed, was that, unlike other communication tools such as radio, TV and mobile phones, computer ICT required constant hard- and software upgrades. These were said not to be cheap and, therefore, posed a cost implication, which might not be affordable by most:

So you find then that is a tool that is constantly upgraded and so there would be a need for that such that we are not using really old computers the market has gone way beyond; some kind of software upgrades would be important (RS3_F).

In addition, another scientist commented that computers were prone to viruses which could lead to computer crashes, data loses and frustration. Thus, having a computer went hand in hand with a need for backups and anti-viruses that also required constant updating. Participants felt this might well discourage adoption of the technology. The virus threat was a major concern in all the public institutions visited under the study, as one scientist noted:

...sometimes scientists get very frustrated if the computer breaks down or something crashes. You know there are things like viruses and things like these... that is why they have been initiating these backups ... (RS2_D)

Moreover, a communications official commented that the computer was susceptible to environmental factors like high humidity and salinity, requiring special handling and storage in air-conditioned premises. He said this increased the maintenance cost in institutions with already limited or no maintenance budgets.

Some participants talked of health-related issues like eye problems and infertility claims that hinder ICT adoption (see 6.1.1.2). Other reported disadvantages inhibiting the adoption of the computer-mediated technology included its dependence on 
unavailable or unreliable infrastructures such as electricity. Participants contrasted this with the mobile phone, which was easy to charge using batteries and solar power at the local grocery shops or neighbouring houses. They added many Kenyans did not have computers and there were few available outlets providing Internet access; hence, the widespread adoption and use of the mobile phone in Kenya, as opposed to computer-mediated communication:

...I want to take up the mobile phone! Why? It is easy to charge. I can charge it with the sun, at the neighbours, local kiosk. And if I want a computer? What will I do with power?...What will I do for Internet if not even available in our local post office and local telecom offices? ... (LR2_A).

Also, participants said that the nature of the computer technology had made it less popular than the mobile phone, whose portability and convenience endeared it to many. The mobile phone could be accessed anywhere, any time, unlike the computer. One interviewee expressed this as: “... the mobile hanging round the neck of my mother ... like an old snuff box” (GO2_Mb). Another participant observed:

... what is actually changing in this country, mode of communication, is actually the mobile telephony. Now you find that almost everybody [has a mobile phone]. You know I have this phone so I can use it here; I can use it even at night even if you call me anywhere I can always use it (LR3_B).

Some suggested that more wireless communication would help increase the adoption of ICT.

Further, as discussed in chapter 6, ICT requires a good level of literacy to be able to make use of it; hence, it is inaccessible to the illiterate (see 6.1.4.3).

As well as inaccessibility to the illiterates, a scientist reported that ICT brought information overload which made it difficult to access useful information:

... information overload and saturation overload is an issue and people must learn to know what they don't have to deal with. For example, email... unnecessary advertisements ... are slowing down access to more useful information... (RS4_E).

He further noted that ICT "also accelerates the access of information that may not yet be published.” This might result in plagiarism or piracy leading to loss of ideas, especially if "ethical issues are not strictly adhered to and followed and IP issues are not respected," he explained. He also said the information might not be fool-proof if proper ways of checking authenticity through peer review had not been followed; thus, allowing unverified or incorrect information to be made available online. 
Another scientist added the plagiarism threat also affected those who had no access to Internet, and still relied on slower, traditional methods of communicating research:

People who have no access to Internet may use the long route, the old route and may not even be able to track down where their publications or drafts are in the fast ICT highway that exists today. So by the time they go back to the old route some ideas could be hijacked by unscrupulous people (RS4_E).

Despite the challenges, participants felt ICT was the way to go and recommended that institutions should facilitate its fast adoption and use through funding, as a scientist commented:

... this [ICT] is something for the new world. We have to use it to make sure we do our things in the fasted way possible ... we have no option but adopt it because it is the way to go. Those who have used it have to testify for this and I find it very useful ...So we need to adopt this and facilitate it through providing money to acquire these things [ICT] (RS2_D).

Thus, the relative advantages were more than the disadvantages.

\subsubsection{Compatibility}

Participants believed ICT was highly compatible with scientific research and that user-friendly interfaces made ICT easy to manipulate for their work, irrespective of discipline:

The Internet technology we have provided for them is compatible with what they do. They don't even have to learn it because the user interface is quite friendly ... (L2_E)

The ICT technology is compatible with almost anything and discipline you would want to use it with if you take the trouble. It has a big advantage over the print (L2_A)

In regard to compatibility, if you look at the sciences, science is actually very compatible with ICT... (LR1_A).

A researcher described ICT as the "best partner in research circles because you will be able to do anything that you want" (RS2_D). He added it is "the best method of communicating or searching for details about their work or some information they need for their research” except for those with limited ICT skills. A library manager summed it thus, “those who go electronic never look back. They don't even know how to look back” (L1_A).

Nevertheless, despite ICT being an enabler for scientific work, participants noted that ICT remained inaccessible to many of the Kenyan institutions: 
We have not reached that level... So the challenge of compatibility is not there (LR1_A)

ICT is an enabler to most of the scientists and very compatible with what we do. The problem is it is not accessible due to problems of connectivity (RS6_C).

The only incompatibility reported by some participants related to the computer brands/systems, whether Macintosh or Intel, and the ignorance of the procurement officers who sometimes acquired equipment that was incompatible.

Thus, compatibility is not a major issue; infrastructure availability is the barrier.

\subsubsection{Complexity/ trialability /ease of use}

Despite the already discussed problems, especially among older researchers (see 6.1.4.1), owing to the newness of ICT and researchers having not reached the threshold level for adoption and use, interviewees reported that ICT was easy to trial out. They believed adoption was likely to increase with greater exposure to the technology, as the excerpts below indicate:

One thing I have observed from this new technology is that by nature it is new and you may not be surprised when you find even these old scientists are finding it difficult to handle that because it is something that has come up recently and like I have seen the people who are better off with this new technology are young scientists... (RS2_D)

For most of the scientists up to the age of 50, it is easy for them to use the technology... (RS6_C).

Participants attributed the ease of use of modern ICT to the user-friendly software that is easy to master if one devoted time to explore the technology:

I think it is very user-friendly if you have the capacity to sit down and actually investigate what it can do for you... with the current softwares ... (LR1_A)

... Most of them find it easy to use. Of course it depends on the technical know-how but I think all those who have taken their time to explore the technology not only find it easy to use but they find it a lot of fun... (L1_A).

Scientists in particular, majority of whom had some training abroad and, consequently, good exposure to ICT, found computer technology easy to use. Many were communicating over the Internet and using ICT for research work:

... they are up-to-date because even most of the programmes [ICT] people are using are relevant to their disciplines and again most of these people have been trained abroad either in Europe, States, Japan; so, they have had good exposure. So they are not using the computers to write letters or word processing. The scientists are really using those relevant programmes to analyse data, come up with drafts, and to present their findings (GO3_F) 
... they [scientists] find it easy to use. We communicate through Internet. We send reports to each other through Internet. So I believe there is a general assumption that it is easy to use and reliable (RS3_F).

A communications official commented that scientists were "the best when it comes to adoption of ICT, some of them even better than IT professionals. We call them almost wizards” (GO3_F). However, a library manager pointed out the exception, with “those who have developed a mental block and they don't want to change” (L1_A). Some other ICT compliant participants made the observation that ICT may be complex to those unfamiliar with the "rigours of this new technology" (GO2_Mb). One researcher commented:

I will not be cheating you and I will tell you actually ease of use of the computer in this institution is still a challenge, not only to the lecturers, even to the graduate students... It takes time; it takes time (LR1_A).

Thus, lack of proficiency could make one give up computer use, failing to achieve the intended efficiency and effectiveness:

... remember if you are not very good in using these computers it can be very frustrating and you may end up giving up. You may also end up not making it actually work the best level or even against you... I have seen people who have actually typed whole documents using one finger like this [demonstrates] all over a sudden find the document is not there because they forgot to save or they deleted it accidentally (LR1_A).

Participants indicated that infrastructural problems, such as non-availability of computers or laptops, made it harder for researchers to familiarise themselves with ICT. Most public institutions were yet to achieve a 1:1 ratio. Moreover, connectivity to the Internet was often lacking, and institutions experienced constant electricity power fluctuations that made it difficult to connect to the server:

The technology itself is easy to use for researchers; what is ... challenging is the availability of the technology ... not many of these people have laptops. If you talk about say somebody should have a PC, the university supplies but I'm not sure where it has reached the ratio of 1-1 for all the lecturers, for all the researchers... I think that is really what kind of pulls them back or ... you can have a computer but probably is not connected to the Internet. ... we have been having problems of power [electricity] ... (LR3_B).

In addition, many public institutional websites were reportedly difficult to navigate. Most lacked links or were still under construction. A scientist reported it was frustrating trying to access them: 
... and may be ease of use could also be the website, ease of the website to navigate. Are the links there? You see by going to let's say a local website that has to be developed further on every link or under construction on every link, that might be the first and last time I may access it (RS3_F).

Thus, difficulties in navigating the websites made them difficult to trial out.

In contrast, participants from the international research institutes did not experience the infrastructural, literacy and connectivity difficulties experienced in public institutions. They said their staff was well prepared for ICT use and the Internet was freely accessible, as one of them reported:

Computer illiteracy is out of question here. There is a very high level of computer literacy. So, on that score we can say we are well prepared for the technology (L1_E).

The only common problem highlighted in both international and national institutions was staff's lack of/need for specialised information search skills (see 5.4 and 7.1.2).

These few noted challenges notwithstanding, it can be concluded that trialability did not pose a major threat to ICT-mediated research communication in Kenya.

\subsubsection{Observability}

It emerged from the findings that the rumour that the initial mainframe computers were locked up in big rooms in the Treasury and at the Chiromo campus of the University of Nairobi (see chapter 6.1.1.2) evoked a sense of mystery and fear; thus, deterring many people from adopting or using computers. However, the desktop computers that followed had made the computer more visible, and, therefore, more acceptable to potential users. This supports Rogers' (2003) claim that the observability of an innovation affects its adoption.

\subsubsection{Reliability}

Participants reported that ICT per se was reliable but the ICT infrastructure in Kenya and Africa in general was not. For example, the electricity system was down most of the time, as a library manager reported:

Generally ICTs are supposed to be very reliable. But then in Africa, as you know, electricity is not reliable. Even as I talk to you now our so-called circulation module is fully computerised [but] we have gone back to manual process because the power is not there, the backup. So again when you come to Africa there are those challenges that we meet and then we describe it as not reliable but in principle it is quite reliable... (L1_A). 
Participants also commented that small bandwidth made it difficult to access the Internet owing to its slow speed; thereby, causing people to avoid the Internet entirely, as the excerpts below indicate:

... bandwidth ... is not reliable. You don't know whether the document is going to open or not. You never know whether you will have access to that website or not; and if you do, you don't know whether you are going to download that document... (L1_A)

It is not reliable because it has a very low speed and you cannot access information any time you want to access... I can say I can access what I want might be for ... between $0-5 \%$ of my time (RS6_C).

This made people lose trust in ICT:

... if your Internet is down most of the time then you also lose faith in the service provider...for many people who might not have a functional Internet then you know when it is down, it is down and is down more often than not (RS3_F).

The Internet's unreliability was attributed to the monopoly of the telecommunications sector by the Telkom Kenya, which limited the ISPs and therefore Internet speed:

The Internet technology was not very reliable... based on the monopoly of our service provider, the Telkom, because many people could not actually provide outside communication... (LR1_A).

Therefore, the reliability problem was largely infrastructural in nature, rather than being ICT-related.

However, a government official suggested the problem might also be researchers' unwillingness to communicate due to their uncertainty about ICT, a problem discussed in detail in chapter 6 (see 6.1.1.2). Similarly, a lecturer mentioned userrelated unreliability, due to lack of ICT skills leading to loss of information and confidence in ICT:

The technology is reliable but remember any equipment is reliable as long as actually the operator is reliable [laughs]. So we could have operator-related unreliability ...you could type and then end up deleting and you are frustrated... It might actually make you lose your confidence in the computer if it happens within the initial stage... (LR1_A).

Another type of unreliability could result from lack of knowledge about which technology works best, which might lead to researchers receiving substandard equipment, as shown by the following comments:

... the computers available here... are branded products, either HP or Dell. So they are actually as reliable and good like any other. The problem is actually if the person 
you decide you are going to buy from decides to cheat you and give you a clone ... (LR1_A)

... the quality in terms [of] once you adopt the technology before you know what suits you to do what work. The donor or salesmen who are bringing you equipment might bring you substandard equipment (GO3_F).

Such issues might discourage many from adopting ICT.

The participants from international and donor-funded national research institutions indicated that they did not suffer the problem of Internet unreliability. One national donor-funded institution had wireless communication, while the international institutions tended to have their own dedicated lines for Internet and standby generators to cushion against the electric power fluctuations; hence, they experienced little down time. As such, the international institutions and the donor-funded national institutions had constant Internet access, except when affected by national issues or the theft of telephone cables, which was reported as frequent.

Participants believed that completion of the ongoing marine optic cable fibre along the East African coast might reduce ICT unreliability problems in Kenya.

From the discussion above, participants had no doubt that ICT had relative advantages over other traditional communication technologies, was easy to trial out, reliable and compatible with researchers' work. It also emerged that, because of the push from the environment, Kenyan research institutions were moving towards universal adoption of ICT. However, lack of adequate and reliable ICT infrastructure remained the biggest challenge.

The next section looks at factors affecting computer-mediated research communication that fall outside the socio-cultural, institutional and technological categories.

\subsection{Other Factors}

Further to the technological factors discussed above, the findings revealed other factors affecting ICT-mediated research communication such as the nature of research discipline/data type; personal or individual institution's initiative; telephone wire thefts and lack of ICT research. These will be discussed in the following sections. 


\subsubsection{Nature of discipline/ type of data}

The findings revealed that the particular nature of the discipline and type of data generated affected ICT-mediated research communication. Some science fields like immunology and software engineering were said to lack people with the necessary competencies/capacities to undertake and communicate research; so, the few scientists in those disciplines had few local peers to engage in research communication with:

... How many people are doing software engineering here so that I can be able to communicate? There is no research to communicate (GO2_Mb)

... lack of equal peers to compete with in research on specific areas because the highly skilled personnel to do that highly skilled research, especially in science, are not many in this country... and they are not many also in the African countries (LR1_A).

Also, some participants noted that scientific research takes time to produce outputs that are valid enough to be communicated. They explained that scientific research goes through a long process of verification, owing to the factual nature of the data, before the data/information could be released for public scrutiny:

... agriculture is slow. If you talk about business and business education they are moving very fast and people move quickly because they learn new tricks and can apply them immediately. But in agriculture you still have a long time before you know something, then you develop a skill for it and you apply it on the ground and get the results... So even if you enhance that in agriculture it will take some time before the results are really communicated (RS1_G)

We will tell them to communicate what is, you know, some way forward even if it is not conclusive. Don't wait for 10 years to communicate but communicate something ... (SE1_C).

This concurs with Borgman (2007), in that the factual status of scientific data makes it hard to disseminate, because some elements are uncertain and unverified (or unverifiable) while others are reliable and valid, calling for continual data interpretation in the research process (Borgman 2007). Moreover, some scientific outputs are in numerical data or samples, and their factual nature takes time to verify. Communicating research output in such form to non-scientific audiences is challenging and demands scientists be trained how to make data transferable/accessible; thus, confirming Borgman (2007): “Access mechanisms vary considerably by the type of data. In some cases data are transferable as artefacts (for example samples or numerical data), and in other cases access requires training someone in a novel technique” (p. 174). 
Due to difficulties in coming up with tangible research outputs that could pass as discoveries, a communications official indicated that researchers tend to hoard research results until they were sure their claims were certified and had claimed ownership through publishing or patenting:

... after a researcher goes to the field and gathers the data, it is like people don't want to release their findings before may be they have done one or two papers out of it which is published may be in recognised journals. So there is that element. (GO3_F).

These findings confirm Borgman's (2007) claim on subjects like Biotechnology, which insist on patenting before publishing whereas others insist on publishing first, thereby, delaying relaying of scientific research results.

The nature of discipline had also been limiting in relation to locally available channels of publishing research outputs like journals, in that participants indicated there were few avenues for publishing local scientific outputs, as explained a library manager:

... the subject orientation or coverage of the journal is also a factor (L2_E).

Another research discipline-related barrier to ICT-mediated research communication and transactions was the practical nature of science subjects like agriculture, which meant scientists tended to spend more time on the ground than online, as a renowned scientist observed:

... agriculture being a very practical subject, the amount of the work they have to do on the ground in order to develop the skills is much more important than tuition they can get online. You can read and read a lot of things but when you do them is very different (RS1_G).

Furthermore, the lengthy verification process of scientific outputs meant that scientists often did not expose them to online channels. This tends to support Borgman's (2007) assertion that not all scientific knowledge can be made digitally mobile, because some essential knowledge will remain "excludable" from representations, as in the case of specialised laboratory mice and cell lines that may not be transferable between laboratories, unless accompanied by students or postdoctoral fellows with the appropriate expertise.

\subsubsection{Personal/individual institutions' initiative}

Participants reported that personal initiative, be it at the individual or institutional level, played a significant role in the adoption and use of ICT, described by one scientist as "self- learning to deal with alternatives [referring to available free ICT 
software] is an issue” (RS4_E). The majority of interviewees mentioned that their computer literacy was the result of personal effort and commitment to learn, as the quotes below from a lecturer, research communication and government official indicate:

I have become computer conversant really through self-driven initiative (GO1_Ma)

... everything I know about computers I taught myself (LR1_A)

Internet is assisting [publishing] but remember at the end of the day it goes to the individual initiative ... (GO3_F).

At the institutional level, a lecturer highlighted the lack of push by the research community for research management or teaching information systems (see chapter 7.1.2.5). This illustrated the lack of self-driven initiatives to adopt ICT for research communication on the part of professionals or individual institutions. The findings affirm earlier studies in Kenya on ICT adoption in public university libraries (Mutula , 2001; Odero-Musakali and Mutula 2007).

Consequently, there was need for self-drive if a culture of ICT-enabled research communication was going to flourish and have impact, since the exponential growth of ICT-mediated communication required strong personal commitment to find out what was new and applicable to one’s situation.

\subsubsection{Telephone wire thefts}

As well as lack of self-driven initiatives, interviewees from both the national and international research institutions reported thefts of copper telephone cables as interfering with their Internet access:

... we have had our copper wires stolen ... making us sometimes go for a whole day before it is fixed again (L2_E)

... [Internet] is quite reliable unless the cables are physically chopped, and occasionally that is done... (RS4_E).

Participants felt there was need for an alternative solution to overcome Internet inaccessibility resulting from such thefts.

\subsubsection{Lack of ICT research}

The findings revealed that the lack of ICT research in Kenya, which would point to gaps on where and how ICT could be applied, could be affecting ICT-mediated 
research communication. A research communications official and a government official, for example, reported there were currently no studies about whether and how Internet was being used to access information:

What we are not sure about at least from IECT (Information, Education and Communication Technology) is whether Internet is facilitating a lot of access to information, whether it is being used for that (GO1_Ma)

What research now can I communicate in the area of technology if there is none? What about IT? What kind of research in IT? (GO2_Mb).

A library manager expressed the need for research on ICT to highlight its role in industrialisation and development, which would, in turn, sensitise national leaders to appreciate and adopt ICT for developmental processes:

Probably they [researchers] could help us by focusing on the research in this area [ICT] to show that actually with ICT and industrialisation as a whole you can increase productivity. I think a lot of researchers of course focus on more urgent things like health - disease eradication and all that. But I think if they could also focus on the role of ICTs in development or role of industrialisation in development and try to prove that you actually increase your productivity and hence improve everything else in the process ... (L1_A).

The gap in research in ICT-mediated research communication was reinforced by another library manager, who felt this study was timely:

It was good brainstorming and trying to bring some thinking in this area of ICT, which I think is ... a very important area because now it is the scientists...[who] have realised what they are doing is not really reaching out where they want it to be. So I think it is a very important area that you are researching (L2_E).

A senior scientist cum science editor confirmed the gap and importance of this study, adding that it should have been undertaken by a team of government experts. He commented the findings could serve as a working document for the government:

Realising how important your project is going to be in this country... that means that your design is very original ... you know the government should actually support you to do this research as their project if they are really smart. ... The government should have actually got up a team of experts to address the issue you are trying to address in your thesis. So your thesis can be even a working document for the government ... for them now to say how do we now incorporate ICT in our government system so that we can move in the 21st. century? (RS5_D).

More research into ICT and research communication might identify the knowledge gaps that ICT application could resolve and, in the process, increase ICT adoption and use for research communication. 


\subsection{Chapter Summary}

The chapter has addressed factors related to the nature of the technology and others outside the socio-cultural, institutional and technological environment, which affected the adoption of ICT for communication of scientific research outputs in Kenya. The findings affirmed Rogers' diffusion theory (Rogers, 1995, 2003) that perceived attributes of any innovation, in terms of relative advantage, trialability, compatibility and observability, affected its rate of adoption. They also revealed the unique situation of these dimensions insofar as researchers' use of ICT in Kenya is concerned and also identified relative disadvantages unique to computer-mediated technology, which have affected its adoption and use by researchers in the Kenyan situation (see table 8.1).

Table 8.1: Summary of Technological Factors

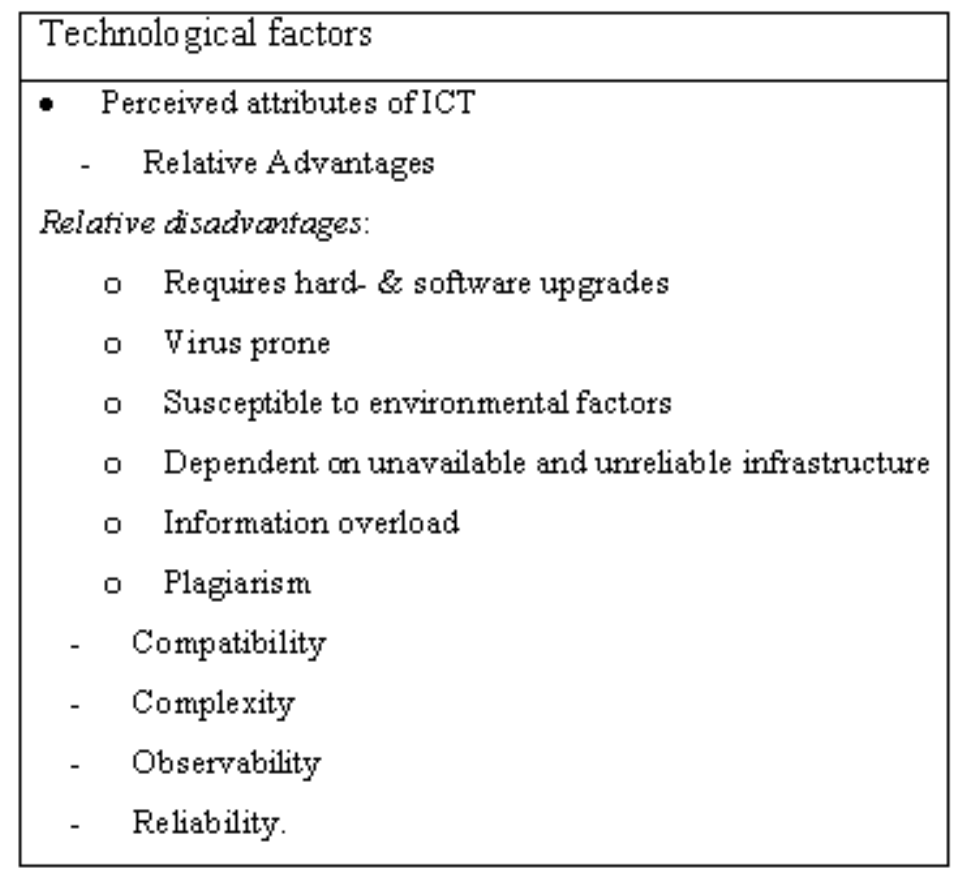

It would be important to take note of all these factors when developing a contextual framework for ICT adoption and use (see figure 8.1). 
Technological factors

- Perceived attributes of ICT

- Relative Advantages
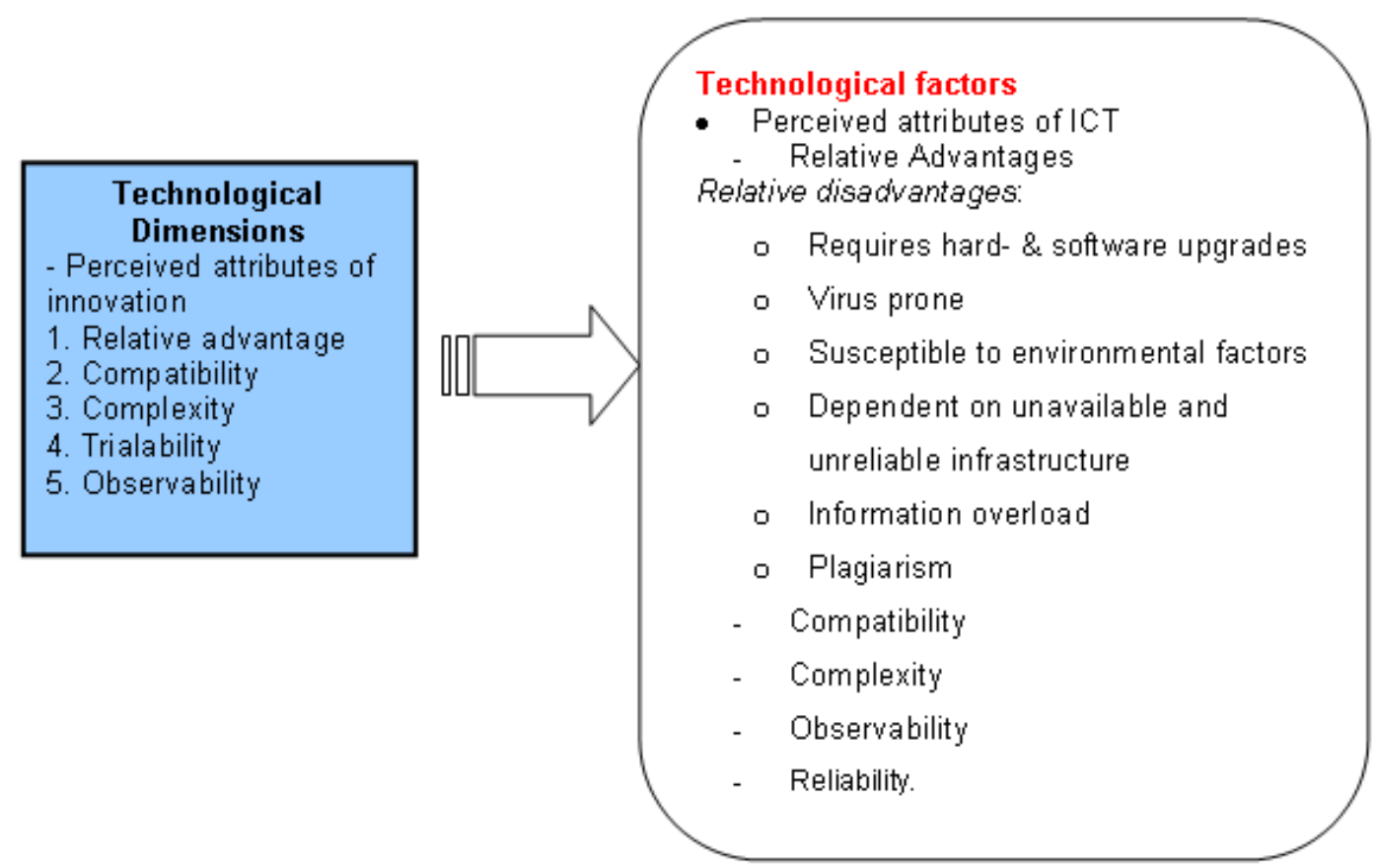

Figure 8.1: Revision of technological dimensions

Moreover, the chapter revealed other factors such as the nature of research discipline/data type, personal initiative, telephone thefts and lack of research on ICT, as also affecting the adoption of ICT for research communication in Kenya (see table $8.2)$.

Table 8.2: Other Factors

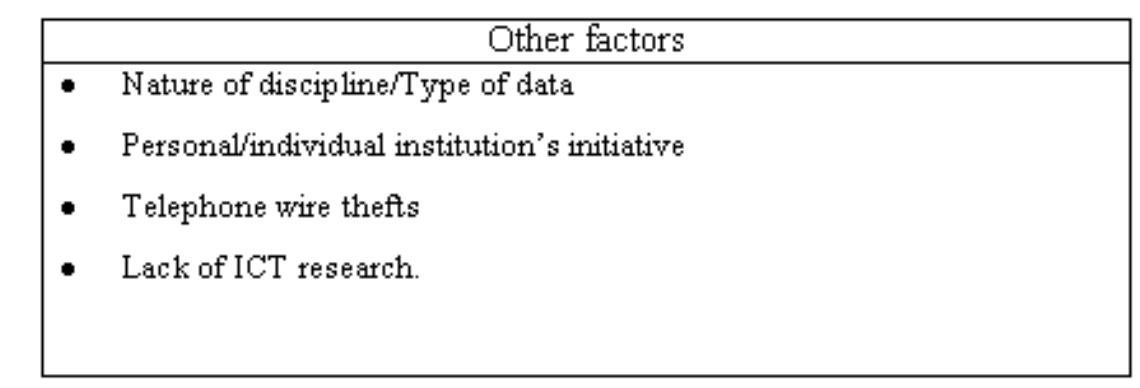

These are factors that the preliminary model did not point to but which emerged from the data; thus, necessitating the revision of the model to accommodate them, for they affect the adoption of ICT in the specific context (see figure 8.2). 
Figure 8.2: Revision of Other DimensionsiFactors

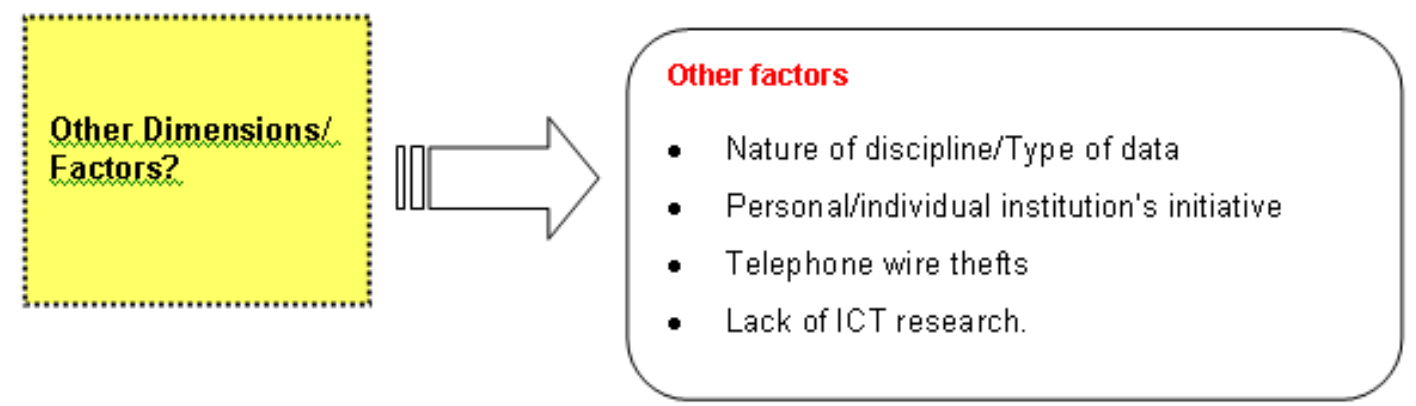

Figure 8.2: Revision of other dimensions/factors

The next chapter briefly reviews the findings and presents a contextual model for ICT-enabled research communication by researchers in scientific research institutions in Kenya, as emerged from the data. 


\section{CHAPTER NINE}

\section{A CONTEXTUAL FRAMEWORK, RESEARCH CONCLUSIONS AND IMPLICATIONS}

\subsection{Chapter Overview}

This chapter concludes the research activities outlined in the research design and methodology in chapter four. First, it highlights the major findings in relation to the research objective and questions and briefly comments on the points of convergence and divulgence from the preliminary theoretical model presented in chapter three. Secondly, it summarises dimensions and factors as revealed by the findings, to address the gaps between theory and practice, and gives a diagrammatic presentation of the revised framework. Thirdly, it presents the research conclusions, its contribution to theory and practice and the study's implications, followed by recommendations and concluding remarks.

\subsection{Data Analysis and Discussion Overview}

This study sought to establish the barriers and enablers to ICT-mediated research communication in Kenya with a view to developing a contextual framework for ICT adoption by and for researchers in applied science research institutions. A preliminary model to meet the objective of the study was developed as a lens to guide the collection of data in relation to issues in the social, cultural, institutional and technological environment affecting researchers' adoption of ICT for research communication.

The previous four chapters analysed and discussed the research findings to answer the four research questions the study sought to answer.

Chapter 5 - reinforcing capacity for strategic research communication - answered RQ1 and 2:

- What are the research communication needs for researchers in Kenya in agriculture, biology/biotechnology, environmental science and health sciences from the perspective of key participants (that is, the researchers, ministries/departments, research institutions)? 
- What are the main priorities for research communication of researchers in Kenya in agriculture, biology/biotechnology, environmental science and health sciences?

The chapter addressed the research communication needs of researchers in the applied sciences in Kenya emanating from their broad socio-cultural, political and institutional environments. This was necessary in order to determine where the research community was coming from before embarking on any talk about ICT adoption framework. The emerging priority research communication need was reinforcement of capacity for strategic research. Participants said this could be achieved by first of all recognising and prioritising research communication through planning and investing in scientific and technological research and its communication. This would include improving tools and infrastructure, especially ICT-enabled ones, such as Internet connectivity and other e-resources.

Chapters 6-8 presented the findings on factors related to the socio-cultural, institutional and technological environments and others that affected ICT-mediated research communication. This was in response to RQs 2 and 3:

- What are the contextual factors that affect ICT-enabled research communication by researchers in Kenya in agriculture, biology/biotechnology, environmental science and health sciences?

- How do these factors affect ICT-enabled research communication by researchers in Kenya in agriculture, biology/biotechnology, environmental science and health sciences?

Salient issues affecting the adoption of ICT that were unique to the Kenyan situation emerged. Chapter 6 highlighted findings on socio-cultural factors such as appreciation and perception of ICT; attitude of the scientific research community; demographic issues such as age/level of qualification, gender and poverty and literacy levels; communication networks and traditional cultural values such as orature, communalism and education culture. These were intricate issues that the preliminary model based on Rogers (1995, 2003), Hofstede’s (2001) perspectives and extant literature did not highlight, but which are determinant when considering developing an ICT framework for scientific research communication in Kenya. They point to 
specific gaps worth paying attention to in the implementation of ICT-mediated research communication.

Chapter 7 highlighted institutional factors. It revealed the need to address issues to do with ICT governance such as political and institutional leadership and culture; institutional framework; policy and strategy and legal and regulatory framework; and control over mass media communication channels. Inadequate capacity for ICTmediated research communication, for example, lack of human capital, failure to assess researchers' need and institutional e-readiness, inadequate ICT tools and infrastructure and inappropriate content, all impacted on ICT-mediated research communication. Lack of demand for MIS for research and teaching led to few initiatives towards implementing ICT-mediated research communication for nobody was pushing for it. Nor was this helped by inadequate resources, in terms of research funding and time to undertake research and surf the web for information. Lack of recognition and motivation left them researchers struggling for survival, not ICTmediated research communication.

Although within the broad and general category of the preliminary framework, which recognised that an institution's type of innovation decision affected the adoption of new innovation, like ICT, these findings served as a specific illustration of the institutional environment of the scientific research institutions in Kenya. They, therefore, qualify to be added to the revised framework (see chapter 7 figure 7.1).

Chapter 8 addressed factors of the technological environment and other factors that could be affecting ICT-mediated research communication. Except for problems emanating from the socio-cultural and institutional environment, the findings revealed ICT had the perceived attributes of relative advantages, compatibility, complexity and observability, thus, affirming Rogers (2003). ICT was also said to be reliable. The only relative disadvantages reported related to the fact that ICT requires hard- \& software upgrades; is virus prone and susceptible to environmental factors; requires other infrastructure that may be unavailable and unreliable; and could lead to information overload and plagiarism. Nevertheless, overall, the advantages, convenience and efficiency associated with ICT endeared it to the research community and they felt ICT-mediated research communication would be the way to 
go. Hence, the new framework retains the technological features of the preliminary model as reflected in chapter 8 (see figure 8.1).

Other emergent factors outside the preliminary model identified in chapter 8 included the nature of discipline/type of data; personal/individual institution's initiative; telephone wire thefts and lack of ICT research. They add a new dimension to the preliminary model and to the study as well. The new framework is adjusted to reflect that (see chapter 8, figure 8.2).

Thus, the Rogers' (2003) and Hofstede’s (2001) diffusion theoretical frameworks just provided a roadmap towards discovering critical contextual issues affecting the adoption and use of ICT for research communication purposes in Kenya.

Table 9.1 gives a summary of all factors as highlighted in the findings in the three chapters that should serve as the building blocks for the revised model framework for adoption of ICT for research communication in Kenya. 
Table 9.1: Summary of Factors to Consider for a Contextual ICT-mediated Research Communication Framework in Kenya

\begin{tabular}{|c|c|}
\hline $\begin{array}{l}\text { Socio-cultural } \\
\text { factors }\end{array}$ & $\begin{array}{ll}\text { - } & \text { ICT appreciation } \\
\text { - } & \text { Perceptions about ICT potentiality and use } \\
\text { - } & \text { Attitude of the research community } \\
\text { - } & \text { Demographic factors } \\
\text { - } & \text { Age/Level of qualification/Exposure } \\
\text { - } & \text { Gender } \\
\text { - } & \text { Communication networks } \\
\text { - } & \text { Traditional cultural values. }\end{array}$ \\
\hline $\begin{array}{l}\text { Institutional } \\
\text { factors }\end{array}$ & 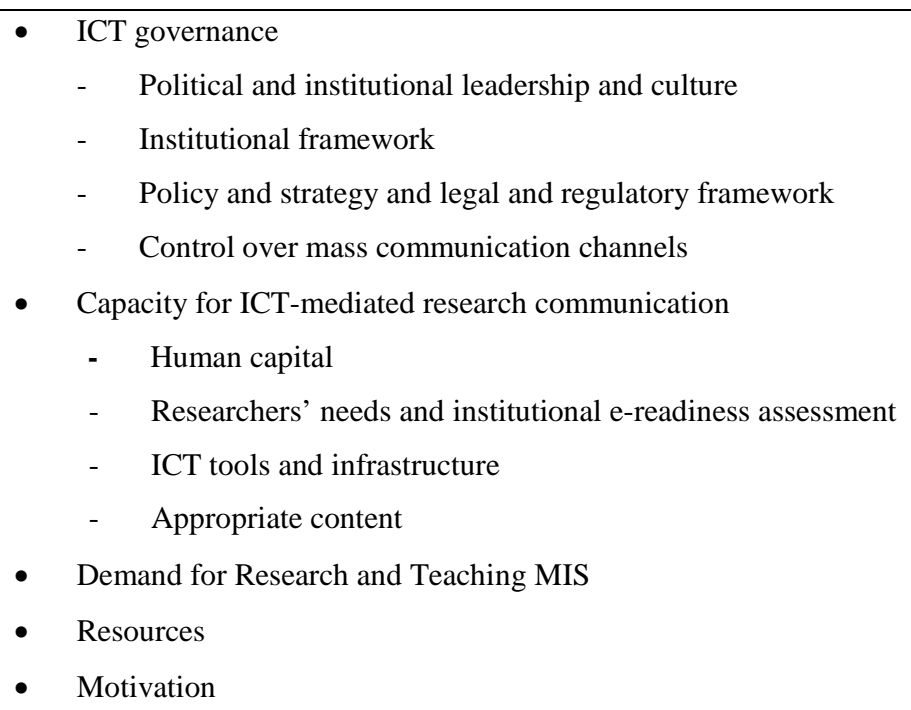 \\
\hline $\begin{array}{l}\text { Technological } \\
\text { factors }\end{array}$ & $\begin{array}{l}\text { Perceived attributes of ICT } \\
\text { - } \quad \text { Relative Advantages } \\
\text { Relative disadvantages: } \\
\text { o } \quad \text { Requires hard- \& software upgrades } \\
0 \quad \text { Virus prone } \\
\text { o Susceptible to environmental factors } \\
\text { o Dependent on unavailable and unreliable infrastructure } \\
\text { o Information overload } \\
\text { o Plagiarism } \\
\text { - Compatibility } \\
\text { - Complexity } \\
\text { - Observability } \\
\text { - Reliability. }\end{array}$ \\
\hline Other factors & $\begin{array}{ll}\text { - } & \text { Nature of discipline/Type of data } \\
\text { - } & \text { Personal/individual institution's initiative } \\
\text { - } & \text { Telephone wire thefts } \\
\text { - } & \text { Lack of ICT research. }\end{array}$ \\
\hline
\end{tabular}




\subsection{A Contextual Framework for ICT-mediated Research}

\section{Communication}

Theoretically informed and richly substantiated by evidence from the findings, the contextual framework of this study is intended to guide implementation and practice of ICT-mediated research communication in Kenya. The purpose of this ICT adoption framework is to assist research institutions to implement and use ICT in an integrated manner, so as to reap the benefits associated with the computer-mediated information communication and in turn boost their research outputs and visibility.

Based on the perceptions of the participants reflected in table 9.1 above, the preliminary model in chapter 3 was revised to reflect a contextual framework for adoption of ICT for research communication in scientific research in Kenya (see figure 9.1 below). 


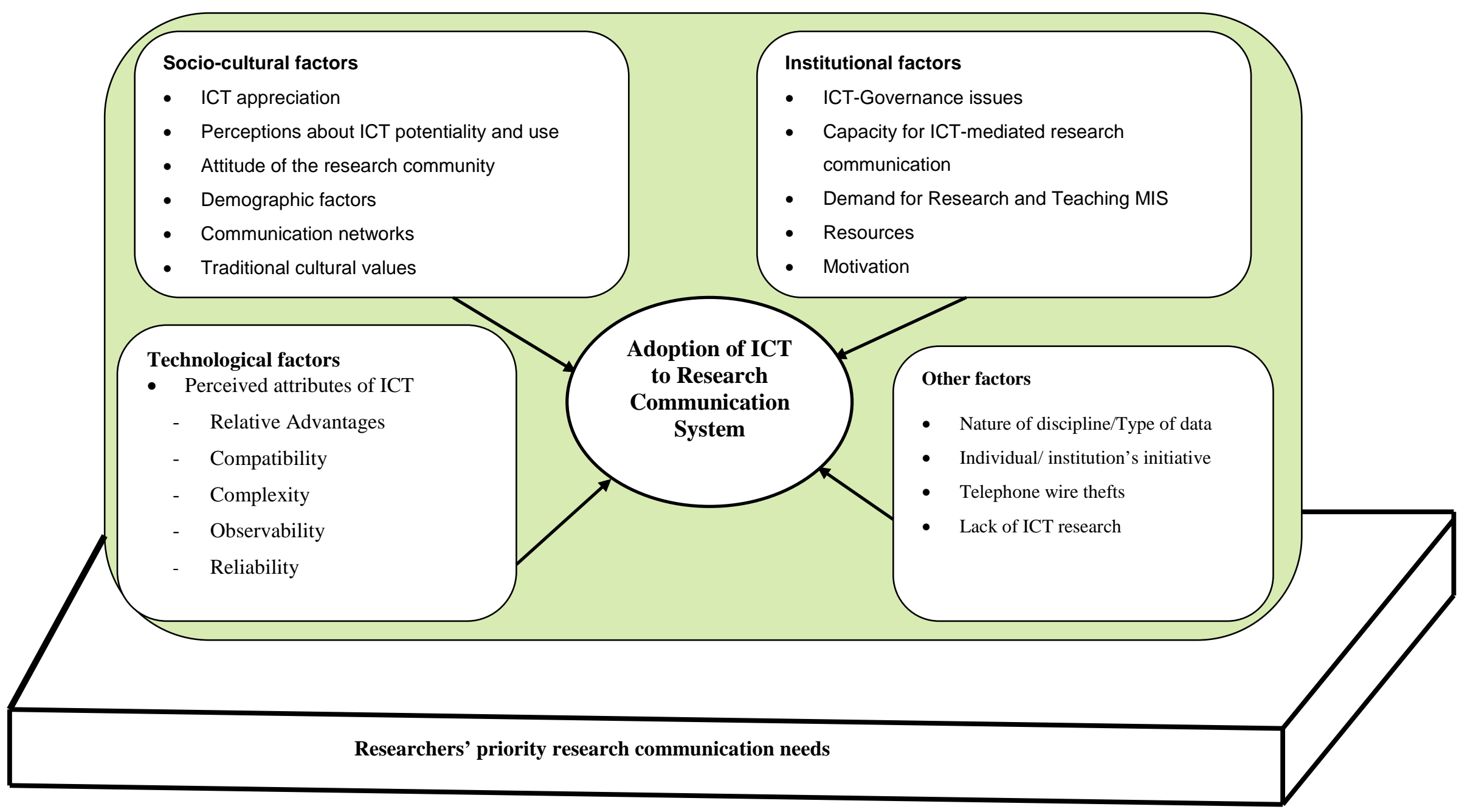

Figure 9.1: A contextual model for ICT-mediated scientific research communication in Kenya 


\subsubsection{Researchers' priority research communication needs}

The literature reviewed stressed the need for ICT adoption and implementation to be in resonance with researchers' priority research communication needs (Beveridge, 2004; Borgman, 2007; Kaniki, 2004). The findings confirmed this and advocated that the researchers' priority research communication needs form the foundation for the contextual model for ICT-mediated scientific research communication in Kenya as integrated in the contextual model above. This will ensure the framework supports strategic ICT-enabled research communication initiatives and guides the integration of research communication requirements and opportunities with ICT adoption. It is only then the communication system can endear itself to researchers and provide the support they need to produce research outputs that can be communicated to inform theory and practice and ultimately contribute to the society's wellbeing.

\subsubsection{Socio - Cultural factors}

The preliminary model based on theories (Hofstede, 2001; Rogers, 2003) and literature (Borgman, 2007; Chakava, 1996; Duque et al., 2005; Gebremichael \& Jackson, 2006; Kling, 1999; Kling \& Callahan, 2002; Zhang, 1998) indicated the need for an understanding of the socio-cultural environment. The findings confirmed this and contextualised these decontextualised psychological constructs by presenting a specific contextual analysis and illustration of the effects of the Kenya socio-cultural environment on the adoption of ICT for communication of research outputs by researchers in the applied sciences institutions. The findings identified distinctive features characteristic of researchers in the Kenyan context such as ICT appreciation and perception; attitude of the research community; unique demographic information on age and level of qualification, gender and poverty and literacy levels; communication networks and traditional cultural values like orature, communalism and education culture.

The specific political and historical situations are illustrations of how Hofstede's (2001) Power Distance, Masculinity/Femininity, Uncertainty/Avoidance and Individualism/Collectivism affect the adoption and diffusion of ICT for scientific research communication. For example, leadership - the power and influence of politics in resource allocation for research where research is marginalized is a form of Power Distance (PD) between the political elite and the research community. It could 
also be illustrative of Uncertainty/Avoidance where the politicians feel safer without intellectuals interfering (Chakava, 1996; Makhubela, 2004) and also without the exposure brought by ICT. This aligns with Hofstede (2001) view on PD in large PD societies like Kenya. High PD was also manifested in the issues of demography like the respect accorded the aged and the highly qualified to a point where they could not be challenged; thus, affecting ICT-mediated research communication.

Poor ICT appreciation due to lack of understanding and fear affirms the literature (Mutula, 2001b; Odero-Musakali \& Mutula, 2007; Ondari-Okemwa, 2002) and align with Rogers (2003) proposition that there was need for champions and early exposure to any innovation for it to be understood well enough to be adopted. Fear resulted from the uncertainty around ICT and how it works. As the findings indicated, ICT threatened the power of those traditionally perceived as knowledgeable; hence, they avoided it (Uncertainty Avoidance).

Unclear perceptions about the functionality of ICT and ICT-mediated research communication led to lack of faith and, therefore, distrust of electronic publications by institutions. This confirmed Hofstede's (2001) UA perspective and Rogers' (2003) claim that complexity of an innovation affected its adoption. However, there was a new dimension to this where participants expressed danger of obsession with ICT tool leading to stifling of creativity, an idea which was supported by the literature (Arunyanak, 2004; Bitman, 2008; Buckingham, et al., 2007; Young, 1998).

Attitude is a factor in the contextual model. The issue of society propagating what it is used to led to non-use of ICT and non-proactivity about its implementation by both individual researchers and institutions. The literature confirmed this (Mutula, 2001b; Odero-Musakali \& Mutula, 2007; Ondari-Okemwa, 2002) and it aligns with Hofstede's (2001) UA perspective and Rogers (2003) that attitude creates a mental block to ICT adoption. Also, there was the issue of attitude of senior ICT illiterate researchers not wanting to learn from their ICT literate juniors and also not communicating research information owing to high PD (Hofstede, 2001). This called for cultural - attitudinal change for faster ICT adoption. 
Demographic factors also affected ICT-mediated research communication in Kenya. Age was a barrier to ICT adoption. Institutions headed by young and exposed leaders were making great advances in ICT-mediated research communication. Older leaders were non-adopters and social inertia pulled them back in that their social status could not allow them to display ignorance or inability to handle ICT (Hofstede's (2001) high PD). However, the findings revealed there was a slow change that was reversing Hofstede (2001) idea of social consequences of high PD due to age/level of qualification and authority in countries of high PD traditions like Kenya - ICT was enabling peer mentoring in areas where seniors could not deliver. This leverages the criticisms of Hofstede's treatment of national culture as uniform and unchanging (Ali \& Brooks, 2009; McCoy, 2003; McSweeney, 2002; Myers \& Tan, 2002; Redding, 1994; Smith, 1998; Sondergaard, 1994; Tayeb, 1994; Winch, et al. 1997; Yeh \& Lawrence, 1995).

Gender emerged as a demographic factor affecting ICT adoption for research communication. This is confirmed in the literature (A Limo, 2008b; UESCO, 2005; Huyer \& Hafkin, 2007; Derbyshire, 2003; Haddad, 2002; Ertl \& Dry burgh, 2005). This resonates with Hofstede (2001) Masculinity/Femininity (M/F) attribute. The initial strong masculinity in Kenya socialised women into believing tough careers like science were a man's domain and the computer "a man's toy.” This socio-cultural inclination left women to pursue soft options of child-minding that contributed to the quality of life for the family but left the tough and time-consuming science education and technology issues to men; hence, the low adoption of ICT by women in general. Nevertheless, the findings reported a gradual reversal of the initial trend and thought (A Limo, 2008b) which disaffirms Hofstede's (2001) views on M/F differences in ICT adoption; thus, giving credence to the views by critics of Hofstede who challenge his view on culture as a uniform and unchanging phenomenon (Ali \& Brooks, 2009; McCoy, 2003; McSweeney, 2002; Myers \& Tan, 2002; Redding, 1994; Smith, 1998; Sondergaard, 1994; Tayeb, 1994; Winch, et al. 1997; Yeh \& Lawrence, 1995). This points to the need for dynamic models to guide IS studies on diffusion of innovation; thereby, justifying the robustness of the contextual model arising from this study for it seems to be addressing a real gap in IS studies. 
Poverty and low literacy were barriers to ICT-enabled research communication, a factor though not explicit in the preliminary model, has been confirmed in the literature (Adams, 1997; Bagchi \& Udo, 2007; Casal, 2007; Gebremichael \& Jackson, 2006; Kaye \& Little, 2004; Kebede, 2004; Mbarika, et al, 2002; Mwesige, 2003; Oyelaran-Oyeyinka \& Lal, 2005; UNESCO, 2002). This could also be argued to be an aspect of relative disadvantage (Rogers, 2003) in that ICT required literacy and affordability for one to be able to use it.

Another aspect emphasised by the contextual model that is viewed as an enabler to ICT adoption is networks and consortia. This confirms Rogers (2003) and goes further to give detailed and unique contextual accounts of consortia in Kenya and the enabling role they were playing in enhancing ICT - enabled research communication. Others in the literature have supported the same (Casal, 2007; Vandoreen, 2002).

Traditional cultural values like communal/oral culture were reported to influence ICT adoption. The silent and individualistic nature of ICT tended to work against people's communal and oral orientation. Though Hofstede (2001) talks of culture affecting a people's use of technology, it could be argued communal/oral aspect was new to theory for it was unique to the Kenyan/African context. The literature confirmed it (Hahn \& Kibora, 2008; Latour, 1988; Ondari-Okemwa, 2002; Borgman, 2007; Bowker, 2005). This called for the need to contextualise culture perspectives as each culture was unique; therefore, strengthening the justification for the contextual model presented by this thesis and the call by Hofstede's critics to view culture as dynamic and context specific (Ali \& Brooks, 2009; McCoy, 2003; McSweeney, 2002; Myers \& Tan, 2002; Redding, 1994; Smith, 1998; Sondergaard, 1994; Tayeb, 1994; Winch, et al. 1997; Yeh \& Lawrence, 1995).

\subsubsection{Institutional factors}

The theories and literature indicated issues of the institutional environment like type of innovation decision and communication channels affected the adoption of new innovations (Damanpour \& Schneider, 2006, 2009; Hofstede, 2001; Rogers, 1995, 2003; Tornatzky \& Klein, 1982). The findings confirmed this. First was ICT governance issues like political leadership. This is a critical factor for it gives direction on integration of new innovations like ICT into work processes and allocates 
resources to facilitate implementation. Consequently, an enlightened and proactive leadership is determinant in ICT adoption, and the literature attests to this (Moon \& Bretschneider, 2002; IT Governance Institute, 2003; Nystrom, Ramamurthy \& Alla, 2002; Oyomno, 2006; Thong \& Yap, 1995). Alongside leadership is the need for a strong policy, strategy, legislation and regulatory framework. The literature affirms this (Bowman-Ngaruiya \& Waema, 2006; Kaniki, 2004; Mutula, 2001b; NdedeAmadi, 2006a; Odero-Musakali \& Mutula, 2007; Ondari-Okemwa, 2002; Sihanya \& Odek, 2006; Wafula \& Etta, 2006). The policy and regulatory environment in Kenya was said to be restrictive. This could be explained from Hofstede's (2001) UA where decision-makers would rather avoid ICT implementation for they were not clear about its functionality and potentiality.

Rogers (1995, 2003) indicates institutional factors like awareness about any new innovation affect its adoption. The findings confirmed this in that low individual institutions' and researchers' capacity for ICT-enabled research communication was a barrier to ICT adoption for research communication. Most researchers' ICT awareness and skills were low and the literature attests to this (Bagchi \& Udo, 2007; Casal, 2007; Durrant, 2004; Mbarika, et al, 2002; Muinde, 2004; Mutula, 2001b; Odero - Musakali \& Mutula, 2007; Ondari-Okemwa, 2002; Oyelaran \& Adeya, 2002; Oyelaran - Oyeyinka \& Lal, 2005; Wafula \& Etta, 2006).

Poor communication skills by researchers also affected ICT-mediated research communication. This was confirmed by the literature (Adewuyi , 2008; Codata, 2007; Gewin, 2009). Coupled with this was researchers' lack of e-readiness. Rogers (1995, 2003) talks of threshold levels before a new innovation can be adopted. The need for e-readiness was highlighted by others (Duque, et al., 2005; Keengwe, et al., 2009; Musa, et al., 2005; Ndede-Amadi, 2006a; Ondari-Okemwa, 2002; Onu, 2008). Further, lack of ICT tools and infrastructure, including cabling in old and new buildings, discouraged ICT-enabled research communication. This was confirmed in the literature (Bagchi \& Udo, 2007; Bowman-Ngaruiya \& Waema, 2006; Casal, 2007; Government of Kenya 2006, 2007a; Rosenberg, 1998, 2005; Wafula \& Etta, 2006). The lack of ICT tools and infrastructure could be explained from Hofstede's (2001) view of high PD in African countries. Unlike in low power distance countries in the West where relationships are horizontal and free communication is valued as a result 
of which they put communication structures in place, African countries have vertical relationships resulting in controlled communication and, therefore, less emphasis on communication structures.

Another issue to do with institutional capacity was lack of adequate and appropriate content that could be communicated. Borgman (2007) and A Limo (2008a) attest to this lack. Although content was not highlighted by the theories, it emerged as a critical contextual barrier to ICT - enabled research communication. Moreover, respondents reported a lack of demand for research and teaching Management Information Systems. This aligns with what Oyomno (2006) calls inadequate functional capability which needs to be built to enable researchers to efficiently apply ICT-mediated research communication. Inadequate resources, and especially funding to facilitate research development, were said to be a major barrier to ICT-enabled research communication. These are contextual issues that need to be reflected in the contextual model.

Motivation emerged as a barrier to ICT-enabled research communication. Unlike in the developed world where researchers are well motivated and facilitated to pursue higher professional status, this was not the case in Kenya and Africa. Researchers were said to be struggling for basics, survival and so did not have time to invest in research communication. Studies have confirmed this (Casal, 2007; Dimaggio, Hargittai, Neuman \& Robinson, 2001; Gebremichael \& Jackson, 2006; Kaniki, 2004). The poor motivation that hinders higher professional pursuit could be said to be a form of PD (Hofstede, 2001) for it this rests with national and institutional leadership.

\subsubsection{Technological and other factors}

Rogers (2003) indicated perceived attributes of a new innovation influence its adoption. The findings revealed that perceived attributes of ICT affected its adoption for research communication. It emerged that lack of ICT infrastructure, computers, small bandwidth that affected Internet reliability reduced the relative advantages of ICT to relative disadvantages (Beveridge, 2004; Durrant, 2004; Kaduda et al., 2005; Ondari - Okemwa, 2002; Osanjo, 2009; Ouya, 2006; Rosenberg, 2005; Shibanda, 2006; P Smart, 2003). 
Other factors that affected the adoption of ICT for research communication included nature of discipline/Type of data in that not all scientific data samples can be represented electronically with some calling for verification while others call for continual data interpretation in the research process(Borgman 2007). This concurs with Borgman (2007). In addition, respondents reported lack of personal/institutions' initiative in ICT use. The exponential growth of ICT-mediated research communication calls for self-learning and drive if a culture of ICT-enabled research communication was to flourish and have impact. Mutula (2001b) and Odero-Musakali \& Mutula, 2007 attest to this. Further, telephone wire thefts and lack of ICT research were reported to affect adoption of ICT for research communication in research institutions in Kenya.

\subsubsection{Section conclusion}

From the aforegoing, it is evident that the contextual model for ICT-enabled scientific research communication in Kenya is robust in that it draws from theory, literature and is formed in the Kenyan context using context specific data where care has been taken to safeguard its integrity by employing a mixture of methodologies and reflexive approach in data gathering, analysis and interpretation to bridge any weaknesses due to choice of theory and design. The interpretivist stance adopted provided rich data to confirm and/or disconfirm the validity of the decontextualised theoretical constructs adopted for this study and, therefore, helps contextualise them. Thus, as much as the research was not testing the constructs as Hofstede (2001) and Rogers (1995, 2003) were, it nevertheless reaffirms the validity of the dimensions; thereby, realigning the findings of the study with theory. Therefore, it could be argued that even though the study cannot be generalised to populations owing to the limited sample and sampling procedure employed, it could be generalised to theory, other similar cultural contexts, and to other developing countries facing similar issues/challenges. This view is in agreement with views expressed by Bryman (2004) and Williams (2000).

The next section concludes the study by discussing its contributions, limitations and implications. 


\subsection{Study Conclusions}

The study set out to explore factors that affect the adoption of ICT for research communication by researchers in scientific research institutions in Kenya with a view to developing a contextual framework to guide ICT-mediated research communication. The goal has been met and the model framework presented in the previous section 9.2. The subsequent sub-sections reassess the study.

\subsubsection{Theoretical and Practical Contributions}

The study has both theoretical and practical contributions. In terms of theory, this study was informed by two robust theories - Rogers' (2003) DOI and Hofstede's (2001) Cultural Dimensions theoretical perspectives. This gives the study and findings breadth and depth, for, as Silverman (2001) said, "Theory, then, should be neither a status symbol nor an optional extra in a research study. Without theory, research is impossibly narrow. Without research, theory is mere armchair contemplation” (p. 110). Using theory to examine the practice broadens the world view and helps in understanding the gaps in the phenomena being investigated. Thus, the study has contributed to an extension of knowledge about existing diffusion theories and cultural perspectives in IS studies.

There is a dearth of literature on interpretive studies in IS using the DOI and Hofstede's cultural perspectives. Therefore, by using the two theories which are largely associated with quantitative studies in this interpretive study, it has helped bring out useful perspectives on ICT adoption relating to the socio-cultural, institutional and technological environment that are aligned to theory. This adds to cases on ICT-adoption studies in IS using theories with a quantitative orientation interpretively. Thus, the study could be said to have a theoretical contribution in that it sheds new light to existing theories.

Further, as already discussed in sections 9.2.1 - 9.2.4, the findings in this study have served to contextualise the decontextualised theoretical constructs of Hofstede (2001) and Rogers (1995, 2003), bringing in new perceptions about the constructs when studied under different and specific contexts. The Kenyan example presented has the potential to provide important new perspectives on IS issues, as well as contributing 
to policy makers, researchers and other researchers in similar situations internationally and in Kenya itself.

The goal of the study was to explore barriers and enablers to adoption of ICT for research communication in Kenya with a view to developing an ICT-enabled research communication framework. The findings from the analysis have added new contextual knowledge regarding diffusion of ICT for research communication within the unique socio-cultural, institutional and technological context of researchers in Kenya. There has been no previous research to this effect. Significant too is the resultant contextual framework for ICT adoption for scientific research communication in Kenya (see figure 9.1). This is important because the diffusion models derive from the developed world context and so do not reflect the complexities of ICT adoption and diffusion for complex and intricate processes like research communication in a developing African country like Kenya. It is such gaps that were precursors to the new contextual framework.

The framework contributes greatly to theoretical understanding of how adoption and diffusion of ICT to scientific research communication can be enhanced in Kenya. The framework's principal dimensions and factors denote unique and key aspects that need to be taken into consideration to facilitate successful implementation of ICTmediated research communication which the preliminary model did not address. These include the need for:

- Strategic ICT-enabled research communication, which should be based on researchers' research communication needs and priorities within the institutional mandates and philosophical orientations

- An understanding of factors of the socio-cultural environment, such as ICT appreciation and perceptions, attitude, demography, communication networks and traditional cultural values; institutional factors such as ICT-Governance, capacity for ICT-mediated research communication, demand for Research and Teaching MIS, resources and motivation. In addition to these are factors such as nature of discipline/type of data, individual/ institution's initiative, telephone wire thefts and lack of ICT research, and technology characteristics.

It is evident from the above that ICT adoption is not generalisable, justifying the need to develop context specific framework for ICT adoption that integrates all the 
components to be able to facilitate ICT-enabled research communication and realise the envisioned outcomes.

On the practical side, the study was timely because it was a response to continuing interest in and dearth of literature about ICT diffusion research in developing countries, especially Africa. It contributes a new wealth of knowledge and data to the ICT diffusion literature in Kenya and Africa in general. The study filled a gap in knowledge and led to identification of further gaps (see 9.3.3).

For the Kenyan government, donor agencies and NGOs, the framework can serve to enrich the ICT implementation planning and decision-making process. It has been informed by not only empirical research but also an extensive review of literature, including white papers on ICT implementation and governance. This should ensure future initiatives in ICT-mediated research communication are focussed to maximise on meagre resources to get the most out of ICT investments.

\subsubsection{Implications of the Research}

The study points to the need for the Kenyan government to reinforce capacity for strategic research that would lead to production of research outputs that could be communicated. This means funding research, providing communication tools and infrastructure.

An e-readiness assessment in all research institutions is necessary to provide baseline information for evaluating ICT adoption for research communication. This would establish a point for comparison and a justification as to why such adoption is necessary.

The findings call upon institutional leadership to become engaged in ICT governance issues and drive ICT-enabled research communication. Such commitment and involvement by the leadership would encourage them to make and be accountable for decisions regarding implementation of ICT-enabled research communication. This would facilitate integration of ICT as an enabling tool that could help institutions realise their goals and mandates and improve the efficiency with which they provide information, in order to inform policy and decision-making procedures and practice. Decisions on implementation of ICT-enabled communication should be the 
responsibility of the leadership in consultation with the professional staff of and the ICT personnel or IT departments. All need to work in consultation to ensure ICTmediated research communication initiatives are in line with the institutional goals and mandates and contribute towards realisation of the greater national and global goals.

A clear institutional framework is mandatory for the success of ICT-mediated research communication in Kenya. The findings point to the need for strategies and institutional frameworks and mechanisms to direct ICT-mediated research communication which should include:

- enforcing a requirement for researchers to communicate research outputs

- linking researchers with the community - the beneficiaries of their research

- establishing centres of excellence to act as act as think tanks and work closely with government, industry, the private sector and the community to spearhead ICT-mediated research communication to ensure it addresses strategic national development issues and global concerns like the MDGs.

The findings pointed to the need to review existing prohibitive government policy regulations and legislation. First was the need to revise institutional mandates to eliminate ambiguities regarding research communication in order to empower researchers to participate in dissemination and implementation of their research findings. The OSA 1968 needs to be revised, and the Freedom of Information Bill fast-tracked, to facilitate free flow of research information. Also necessary is the enactment of a new legislation that would recognise and validate the ecommunication/publishing of research outputs, as well as strengthening current IPR laws and policies to safeguard Kenya's limited scientific discoveries in the fast changing global environment made possible by ICT. It is also crucial to finalise the STI policy to guide implementation of ICT-mediated scientific, technological and innovative research.

The revised framework developed by this study illustrates the necessity for all components to be taken into account when planning for and implementing any ICTmediated research communication system. The findings revealed that the various 
dimensions are interdependent and affect each other and the smooth implementation of ICT-mediated research communication.

The findings pointed to the need for wide-ranging promotional activities to create awareness about the computer-mediated communication and educate the research community and research beneficiaries about its potentialities. Hand in hand with this should be building communicative and ICT skills, to help overcome social inertia and technophobia and attract more researchers, especially the more traditional, older ones, to use ICT.

\subsubsection{Recommendations for Future Research}

Though most participants talked of computer-mediated ICT as having a relative advantage over other traditional ICTs like the radio, barazas and paper-based technologies, some felt traditional methods of disseminating research outputs like the baraza and the radio might still be better suited to Kenya and other African countries, owing to the oral/communal nature of the people and the infrastructural difficulties currently experienced. On the basis of this, this study invites further research on the assessment and evaluation of the relative advantage of ICT over other traditional communication technologies in research communication in developing countries. Such an assessment would help give an understanding of which relative advantages of ICT are most significant to researchers and end-users. Based on such an assessment, research institutions would be able to advise researchers and their clientele to capitalise on the most significant advantages of ICT.

The findings identified the importance of social systems, such as professional or research target groups' consortia or cooperatives, as enablers for ICT-enabled research communication. This study recommends further investigation on how ICT are supporting/might support communication and collaboration between individual target group consortia, for example, specialised farmers or types of patients, and researchers. This would lead to a more comprehensive and fuller exploitation of ICT in order to inform practice and improve livelihood.

Gender socialisation was also identified as a barrier to ICT-enabled research communication to some extent. Further research could be undertaken to explore how 
gender perceptions affect ICT-mediated research communication, both at university and specialised scientific research institutions.

Finally, this study should be expanded to include other specialised tertiary education institutions, both in Kenya and other Sub-Saharan countries, for a more comprehensive picture of the factors affecting the adoption of ICT in the Sub-Saharan African region. This would help address real issues in implementing ICT-mediated research communication, not symptoms.

\subsubsection{Concluding Remarks}

The problem investigated in this study was the lack of understanding of factors of the socio-cultural, institutional and technological environment that affected ICT-mediated research communication in research institutions in Kenya. A framework for ICTmediated research communication was also missing.

Previous studies pointed to a dearth of literature, especially in ICT-mediated research communication, and called for empirical and theoretically informed research to fill the gap. Given that most ICT diffusion studies have been largely quantitative, the studies also pointed to the need for interpretive study to give an in-depth analysis of the situation.

Informed by diffusion theories and empirical findings, the study has developed a contextual framework to guide implementation of ICT-enabled research communication in Kenya. Although the framework's dimensions are conceptually high level and the factors can apply in many institutions with similar backgrounds, it has limited generalisability due to the interpretive nature of the study. However, owing to the fact that the framework is theoretically informed and not merely based on the field findings, it could be nevertheless generalised in that sense.

Pending further research to test and confirm the robustness of the framework, it could offer a distinct contribution to empirical knowledge about the adoption of ICT for research communication in developing African countries. It builds upon prior theoretical understanding of how new innovations like ICT diffuse and are adopted and gives a roadmap to guide implementation of ICT-mediated research communication. 
Finally, the researcher can only hope that the study, in keeping with Newton's acclaim (Meadows, 1998), provides the shoulders for others to step on in the quest to find answers to the many issues affecting the adoption and diffusion of ICT for scientific research communication, especially in Africa. May the journey start where this one has stopped... 


\section{REFERENCES}

Abrahamson, E. (1991). Managerial fads and fashions. The diffusion and rejection of innovations. Academy of Management Review, 16(March), 586-612.

Adams, L. (1997). Internet connectivity and social choices in Africa. Africa Communications, 8(1), 16-19.

Adewuyi, D. (2008). African scholars publishing in American online journals: An empirical analysis by an editor. Paper presented at the Conference on Electronic Publishing and Dissemination "Putting African Journals On Line: Opportunities, Implications and Limits", Dakar, Senegal, 6-7 October 2008.

Adeya, C. N., \& Oyelaran-Oyeyinka, B. (2002). The Internet in African universities: Case studies from Kenya and Nigeria. Maastricht, The Netherlands: United Nations University.

Agarwal, R., \& Prasad, J. (1997). The role of innovation characteristics and perceived voluntariness in the acceptance of information technologies. Decision Sciences, 28(3), 557-582.

Agarwal, R., \& Prasad, J. (1998). A conceptual and operational definition of personal innovativeness in the domain of information technology. Information Systems Research, 9(2), 204-215.

Ajzen, I. (1991). The theory of planned behavior. Organizational Behavior and Human Decision Processes, 50(2), 179-211.

Ali, M. \& Brooks, L. (2009). A situated cultural approach for cross-cultural studies in IS. Journal of Enterprise Information Management, 22(5), 548-568.

Ali, M., Brooks, L. \& Alshawi, S. (2008). Culture and IS: A Criticism of Predefined Cultural Archetypes Studies. Proceedings of the Fourteenth Americas Conference on Information Systems, Toronto, ON, Canada 14-17 August 2008.

Ali, M., \& Brooks (2008). Culture and IS: National Cultural Dimensions within IS discipline. Proceedings of the $13^{\text {th }}$ Annual Conference of the UK Academy for Information Systems (UKAIS), Bournemouth University, 10-11 April 2008, pp. $1-14$.

Alo, M. A. (2003). Communicative competence and academic discourse in an ESL university setting. In L. Oyeleye \& M. Olateju (Eds.), Readings in language and literature (pp. 115-126). Ibadan, Nigeria: Intec Printers.

Altbach, P. G. (1977). Servitude of the mind? Education, dependency, and neocolonialism. Teachers College Record, 79, 187-204.

Altbach, P. G. (1978a). Scholarly publishing in the Third World. Library Trends, 26, 489-503.

Altbach, P. G. (1981). The university as centre and periphery. Teachers College Record, 82, 601-621.

Altbach, P. G. (1982). Higher education in the Third World: Themes and variations. Singapore: Maruzen, Asia.

Altbach, P. G. (1987a). The knowledge context: Comparative perspectives on the distribution of knowledge. Albany, NY: State University of New York Press.

Altbach, P. G. (1987b). The knowledge networks in the modern world. Canadian and International Education, 16(2), 73-88.

Altbach, P. G. (1998). The role and nurturing of journals in the Third World. In P. G. Altbach \& D. Teferra (Eds.), Knowledge dissemination in Africa: The role of 
scholarly journals (pp. pp.1-12). Chestnut Hill, MA: Bellagio Publishing Network.

Altbach, P. G. (Ed.). (1978b). The distribution of knowledge in the Third World: A case study in Neocolonialism. New York, NY: Longmans.

Altbach, P. G. (Ed.). (1992). Publishing and development in the Third World. London: Hans Zell Publishers.

Altbach, P. G. (Ed.). (1993). Third World publishers and the international knowledge system. Buffalo, NY: Bellagio Publishing Network.

Altbach, P. G. (Ed.). (1996). The challenge of the market: Privatization and publishing in Africa. Chestnut Hill, MA: Bellagio Studies in Publishing 7, Bellagio Publishing Network.

Archibugi, D., \& Coco, A. (2004). A new indicator of technological capabilities for developed and developing countries (ArCo). SPRU Electronic Working Paper Series, Paper No. 111, (Paper No. 111). Retrieved 12 March 2007from www.humandevelopment.bu.edu/dev_indicators/show_info.cfm?index_id=36 4\&data type $=1$

Archibugi, D., \& Coco, A. (2005). Measuring technological capabilities at the country level: A survey and a menu for choice. Research Policy, 34, 175 - 194.

Arunyanak, J. (2004). Internet Addiction. 55-60. Retrieved 14 February 2009from www.espuc.east.spu.ac.th/eoffice/journal/June-nov48/55-60.pdf

Ashling, J. (2005). The web and after: The future of scholarly e-publishing Information Today, 6, 33 - 34.

Association of African Universities (2004). AAU Strategic Plan 2003 - 2010. Retrieved 1 April 2006 from www.aau.org

Association of African Universities (2005). The importance of bandwidth to national education, science and technology development challenges. Paper presented at the Conference on African Research and Education Networking Infrastructure, Tunis.

Association of Research Libraries (2000). Principles for emerging systems of scholarly publishing. Retrieved 5 April 2006 from www.arl.org/scomm/tempe.html

Atkins, D., E, Droegemeier, K. K., Garcia-Molina, H., Lein, M. L., Masserschmitt, D. G., Messina, P., et al. (2003). National science foundation blue-ribbon advisory panel on cyberinfrastructure, revolutionalizing science and engineering through cyber-infrastructure: National Science Foundation, USA.

Avgerou, C. (2004). Information technology and data in the context of developing countries. Paper presented at the Open Access and the Public Domain in Digital Data and Information for Science, Paris.

Bagchi, K. (2001). The impact of national culture and other national-level indicators on information technology (IT) diffusion. Unpublished PhD dissertation, Florida Atlantic University, Boca Raton, FL.

Bagchi, K., \& Udo, G. (2007). Empirically testing factors that drive ICT adoption in Africa and OECD set of nations. Issues in Information Systems, 8(2), 45-52.

Bakkabulindi, F. E. K., Nkata, J. L., \& Amin, M. E. (2008). Organizational characteristics as correlates of ICT adoption in Makerere University. Kampala International University Research Digest, 1(2), 124-137. Retrieved 2 February 2009 from

www.ahero.uwc.ac.za/index.php?module $=$ cshe\&action=downloadfile\&fileid $=$ 368071 
Bandura, A. (1986). Social foundations of thought and action: A social cognitive theory. Englewood Cliffs, NJ: Prentice Hall.

Baskerville, R. (1995). Structural entropy model of technology transfer. Paper presented at the First IFIP TC8 Working Conference on Diffusion and Adoption of Information Technology, October 14-17, Leangkollen, Oslo, Norway.

Berner, E. S., Detmer, D. E., \& Simborg, D. W. (2005). Will the wave finally break? A brief view of the adoption of electronic medical records in the US. Journal of the American Medical Informatics Association, 12(1), 3-7.

Beveridge, M. (2004). The ptolemy project: Delivering electronic health information in East Africa. Paper presented at the Open Access and the Public Domain in Digital Data and Information for Science, Paris.

Bittman, M. (2008 March). I need a virtual break. No, really. NY Times. Retrieved 8 March 2008 from http://www.nytimes.com/2008/03/02/fashion/02sabbath.html?pagewanted=2\&_r=1

Boettcher, J. (2006). Framing the scholarly communication cycle. Online, 30(3), 2426.

Borgman, C. L. (2007). Scholarship in the digital Age: Information, infrastructure, and the Internet. Cambridge, MA: The MIT Press.

Bowker, G. C. (2005). Memory practices in the sciences. Cambridge, MA: MIT Press.

Bowman-Ngaruiya, W., \& Waema, T. (2006). The institutional structures and models for implementing the Kenyan national ICT plan. In G. Outa, F. Etta \& E. Aligula (Eds.), Mainstreaming ICT: Research perspectives from Kenya (pp. 25-72). Nairobi, Kenya: MvuleAfrica Publishers.

Bradley, J. R. (1993). Methodological issues and practices in qualitative research. Library Quarterly, 63(4), 431-449.

Brancheau, J. C., \& Wetherbe, J. C. (1990). The adoption of spreadsheet S/W: testing innovation diffusion theory in the context of end-user computing. Information Systems Research, 1, 115-143.

Bryman, A. (2004). Social science research methods (2nd. ed.). Oxford, UK: Oxford University Press.

Buckingham, D., Whiteman, N., Willet, R., \& Burn, A. (2007). The impact of the media on children and young people with a particular focus on computer games and the Internet. Retrieved 20 January 2009 from www.dcsf.gov.uk/byronreview/pdfs/Buckingham Bunker, D., \& Dean, R. (1998). Information technology and systems (IT\&S) as tools: The cultural context of tool creation and use. Foundations of information systems. Retrieved 20 July 2006 from www.cba.uh.edu/ parks/fis/bunker.htm

Canagarajah, A. S. (1996). 'Nondiscursive' requirements in academic publishing. Written Communication, 13(4), 435.

Casal, C. R. (2007). ICT for education and development. Info, 9(4), 3-9.

Castells, M. (2000). End of Millennium - The information age: Economy, society and culture (2 ed. Vol. 3). Malden, MA: Blackwell Publishers.

Chakava, H. (1992). Kenyan publishing: Independence and dependence. In P. G. Altbach (Ed.), Publishing and development in the Third World (pp. 119-150). London: Hans Zell Publishers.

Chakava, H. (1996). Publishing and state censorship in Kenya. Bellagio Publishing Network Newsletter, 16 (Spring ), 14.

Chakrabarty, A., Feinman, S., \& Fuentevilla, W. (1983). Characteristics of sources, channels and contents for scientific and technical information systems in 
industrial R\&D. IEEE Transactions on Engineering Management, EM30(May), 83-88.

Clarke, R. (1999). A primer in diffusion of innovations theory. Retrieved 3 January 2007 from www.rogerclarke.com/SOS/InnDiff.html

CODATA (2007). CODATA task group on data sources for sustainable development in SADC. CODATA WORKSHO. Retrieved 2 February 2009, from www.icsu-africa.org/wsrep_codata_may2007.pdf

Commonwealth of Learning (2000). Identifying barriers encountered by women in the use of information and communication technologies (ICTs) for open and distance learning in Africa. Retrieved 26 May 2006 from www.col.org

Compeau, D. R., \& Higgins, C. A. (1995). Computer self-efficacy: Development of a measure and initial test. MIS Quarterly 19(2), 189-211.

Compeau, D. R., Higgins, C. A., \& Huff, S. (1999). Social cognitive theory and individual reactions to computing technology: A longitudinal study. MIS Quarterly 23(2), 145-158.

Cooper, R. (1994). The inertial impact of culture on IT implementation. Information \& Management, 27, 17-31.

Cooper, R., \& Zmud, R. (1990). IT implementation research. Management Science, 123-139.

Cowan, R. (2004). Economic overview of open access and the public domain in digital scientific and technical information. Paper presented at the Open access and the public domain in digital data and information for science, Paris.

Creswell, J. W. (2003). Research design: Qualitative, quantitative, and mixed methods approaches (2 ed.). Thousand Oaks, CA: Sage Publications.

Dahms, M. (2000). Africa joining the information age - Investigating the need for information and communication systems in rural Tanzania.Unpublished manuscript.

Damanpour, F. \& Schneider, M. (2009). Characteristics of innovation and innovation adoption in public organizations: Assessing the role of managers. Journal of Public Administration Research and Theory 19, 495-522.

Damanpour, F. \& Schneider, M. (2006). Phases of adoption of innovation in organizations: Effects of environment, organization, and top managers. British Journal of Management 17, 215-36.

Darko-Ampem, K. O. (2003). Scholarly publishing in Africa: A case study of the policies and practices of African university presses. Stirling, UK: University of Stirling.

Davis, F. D. (1989). Perceived usefulness, perceived ease of use and acceptance of information technology. MIS Quarterly, 13, 319-340.

Davis, F. D., Bagozzi, R. P., \& Warshaw, P. R. (1989). User acceptance of computer technology: A comparison of two theoretical models. Management Science, 35(8), 982-1002.

Davis, F. D., Bagozzi, R. P., \& Warshaw, P. R. (1992). Extrinsic and intrinsic motivation to use computers in the workplace. Journal of Applied Social Psychology, 22(14), 1111-1132.

Dekimpe, G., Parker, P., \& Sarvary, M. (2000). Global diffusion of technological innovations: A couple-hazard approach. Journal of Marketing Research(February), 47-59.

Denzin, N. K., \& Lincoln, Y. S. (2005). Introduction: The discipline and practice of qualitative research. In N. K. Denzin \& Y. S. Lincoln (Eds.), The Sage 
handbook of qualitative research (3rd ed., pp. 1 - 32). Thousand Oaks, CA: Sage.

Derbyshire, H. (2003). Gender issues in the use of computers in education in Africa. Retrieved 29 February 2009 from www.enawa.org/icons/Tekstbestanden/Gender\%20issues\%20in\%20use\%20of \%20comp\%20in\%20education\%20in\%20Africa\%20Report.doc

Desai, M., Fukuda-Parr, S., Johansson, C., \& Sagasti, F. (2002). Measuring the technology achievement of nations and the capacity to participate in the network age. Journal of Human Development, 3(1), 95-122.

DiMaggio, P., Hargittai, E., Neuman, W. R., \& Robinson, J. P. (2001). Social implications of the internet diffusion. Annual Review of Sociology, 27, 307336.

Drucker, P. (1985). The discipline of innovation. Harvard Business Review 63 (3), 67-72.

Dufborg, A. (2005). Preface. In S. Danofsky (Ed.), Open access for Africa: Challenges, recommendations and examples (pp. iii-iv). New York, NY: United Nations ICT Task Force.

Duque, R. B., Ynalvez, M., Sooryamoorthy, R., Mbatia, P., Dzorgbo, D. S., \& Shrum, W. (2005). Collaboration paradox: Scientific productivity, the Internet, and problems of research in developing areas. Social Studies of Science, 35(5), 755-785.

Durrant, S. (2004). Overview of initiatives in the developing world. Paper presented at the Open Access and the Public Domain in Digital Data and Information for Science Conference, Paris.

Dwyer, S., Mesak, H., \& Hsu, M. (2005). An exploratory examination of the influence of national culture on cross-national product diffusion. Journal of International Marketing 13 (2), 1-27.

Elenkov, D. \& Manev, I. (2005). Top management leadership and influence on innovation: The role of socio-cultural context. Journal of Management 31(3), 381-402.

El-Shinnawy, M., \& Vinze, A. (1997). Technology, culture and persuasiveness: A study of choice-shifts in group settings. International Journal of HumanComputer Studies, 47(3), 473-496.

Esanu, J. M., \& Uhlir, P. F. (Eds.). (2004). Open Access and the Public Domain in Digital Data and Information for Science: Proceedings of an International Symposium. Paris., Washington D.C:. The National Academies Pres.s

Evers, V., \& Day, D. (1997). The role of culture in interface acceptance. Human Computer Interaction, INTERACT'97 Retrieved 20 July 2006 from wwwet.open.ac.uk/pp/v.evers/htmlfiles/home/interact97.htm

Fichman, R. G., \& Kemerer, C. F. (1999). The illusory diffusion of innovation: An examination of assimilation gaps. Information Systems Research, 10(3), 255275.

Fishbein, M., \& Ajzen, I. (1975). Belief, attitude, intention and behaviour: An introduction to theory and research. Reading, MA: Addison-Wesley.

Franks, J. (1993). What is an electronic journal? Public-access computer systems forum. Retrieved 2 May 2006 from www.listserv.uh.edu/cgibin/wa?A2=ind9301c\&L=pacs-I\&P=5953,6067,6181,6295

Gadio, C. M. (2001). Exploring the gender impacts of World Links in some selected African countries: A qualitative approach. Washington, DC: World Links. 
Gebremichael, M. D., \& Jackson, J. W. (2006). Bridging the gap in Sub-Saharan Africa: A holistic look at information poverty and the region's digital divide. Government Information Quarterly, 23(2), 267-280.

Gewin, V. (2009). Transferring skills into career options. Science Careers Retrieved 2 March 2009, from www.sciencecareers.org/businessfeatures

Goodman, S. E. (1991). The globalization of computing. Communications of the ACM, 34, 21-25.

Goodman, S. E., \& Green, J. D. (1992). Computing in the Middle East. Communications of the ACM, 35, 21-25.

Gopalakrishnan, S. \& Damanpour, F. (1994). Patterns of generation and adoption of innovations in organizations: Contingency models of innovation attributes. Journal of Engineering and Technology Management 11, 95-116.

Gorman, G. E., \& Clayton, P. (1997). Qualitative research for the information professional: A practical handbook. London: Library Association Publishing.

Gorman, G. E., \& Clayton, P. (2005). Qualitative research for the information professional: A practical handbook (2nd ed.). London: Facet Publishing.

Goverment of Kenya (2004). E-government strategy. Nairobi, Kenya: Government Printer.

Government of Kenya (1968). The Official Secrets Act Retrieved 10 October 2008. from www.kenyalaw.org/kenyalaw/klr_app/frames.php.

Government of Kenya (2001). Poverty reduction strategy paper (Vol. 1). Nairobi, Kenya: Government Printer.

Government of Kenya (2003a). Report of the national conference on education and training. Paper presented at the Meeting the Challenges of Education and Training in Kenya in the 21st Century, Kenyatta International Conference Centre, Nairobi, November 27 - 29.

Government of Kenya (2003b). Report of the sector review and development. Nairobi: Ministry of Education, Science \& Technology.

Government of Kenya (2004a). E-government strategy. Nairobi, Kenya: Government Printer.

Government of Kenya (2004b). Investment programme for the economic recovery strategy for wealth and employment creation 2003 - 2007 (Revised edition).

Government of Kenya (2005a). Kenya education sector support programme 2005 2010: Delivering quality education and training to all Kenyans. Nairobi, Kenya: Ministry of Education, Science \& Technology.

Government of Kenya (2005b). Sessional paper no. 1 of 2005 on a policy framework for education, training and research: Meeting the challenges of education, training and research in Kenya in the 21st. century Nairobi, Kenya: Ministry of Education, Science \& Technology.

Government of Kenya (2006). The Kenya Gazette. Nairobi, Kenya: Government Printer.

Government of Kenya (2007a). Kenya's Science, Technology and Innovation Policy 2007. Unpublished Draft.

Government of Kenya (2007b). The Kenya Freedom of Information Bill 2007. Nairobi, Kenya: Government Printer.

Government of Kenya (2007c). Kenya ICT Bill 2007. Nairobi, Kenya: Government Printer.

Government of Kenya (2007d). Kenya Vision 2030. Nairobi, Kenya: Government Printer. 
Grbich, C. (2007). Qualitative data analysis: An analysis. Thousand Oaks, CA: Sage Publications.

Grover, V., \& Goslar, M. (1993). Initiation, adoption etc of telecommunications technologies in US organizations. JMIS, 10, 141-163.

Guba, E., \& Lincoln, Y. S. (1994). Competing paradigms in qualitative research. In N. K. Denzin \& Y. S. Lincoln (Eds.), Handbook of qualitative research (pp. 105 117). Thousand Oaks, CA: Sage.

Guedon, J. C. (1994). Why are electronic publications difficult to classify? The orthogonality of print and digital media. Directory of Electronic Journals, Newsletters and Academic Discussion Lists 4th. Retrieved 26 June 2006, from www.people.virginia.edu/ pm9k/libsci/guedon.html

Gurbaxani, V. (1990). Diffusion in computer networks: The diffusion of BITNET. Communications of the ACM, 12, 65-75.

Gurbaxani, V., \& Mendelson, H. (1990). An integrative model of IS spending growth. Information Systems Research(March), 23-46.

Haddad, W., \& Draxler, A. (2002). Technologies for education: potential, parameters and prospects: UNESCO.

Hafkin, N., \& Taggart, N. (2001). Gender, information technology and developing countries: an analytic study: WID Office, USAID.

Hahn, H. P., \& Kibora, L. (2008). The domestication of the mobile phone: oral society and new ICT in Burkina Faso. J. of Modern African Studies 46(1), 87109. Retrieved 10 January 2009 from www.journals.cambridge.org

Hargittai, E. (1999). Weaving the western web: explaining differences in internet connectivity among OECD countries. Telecommunications Policy, 23, 701718.

Hargittai, E. (2001). Holes in the net: The Internet and international stratification. Retrieved 10 November 2006, from www.isoc.org/inet98/proceedings/5d/5d_1.htm

Hart, P., \& Saunders, C. (1997). Power and trust: Critical factors in the adoption and use of EDI. Organizational Science, 8, 23-42.

Harvey, F. (1997). National cultural differences in theory and practice: Evaluating Hofstede's national cultural framework. Information Technology \& People, 10(2), 132-146.

Hasan, H., \& Ditsa, G. (1999). The impact of culture on the adoption of it: An interpretive study. Journal of Global Information Management, 7(1), 32-146.

Hayhoe, R. (1986). China, comparative education and the world order models project. Compare, 16(1), 65-80.

Hayhoe, R. (1989). China's universities and the open door. Toronto, Canada: OISE Press.

Hebert, D. M. (2007). Innovation in teacher education: Faculty members' and assessment coordinators' perceptions of electronic assessment systems. Unpublished $\mathrm{PhD}$ dissertation, Louisiana State University and Agricultural and Mechanical College, Louisiana.

Henry, G. (2003). On-line publishing in the 21st century. D-Lib Magazine, 9(10). Retrieved 29 May 2006 from www.dlib.org/dlib/october03/henry/10henry.html

Herbig, P., \& Muller, J. (1992). Culture and technology: Does the traffic move both directions? Journal of Global Marketing, 6(3), 75-104. 
Hill, C.E., Loch, K.D., Straub, D.W \& El-Sheshai, K., (1998). A qualitative assessment of Arab culture and information technology transfer. Journal of Global Information Management 6(3), 29-38.

Hofstede, G. (1980a). Culture's consequences: International differences in workrelated values Beverly Hills, CA: Sage Publishers.

Hofstede, G. (1980b). Motivation, leadership and organization: Do American theories apply abroad? Organizational Dynamics (Summer), 42-63

Hofstede, G, (1983). National culture in four dimensions. International Studies of Management and Organization, 13(2), 46-74.

Hofstede, G, (1984). Culture's consequences. Newbury Park, CA: Sage.

Hofstede, G, (1991).Cultures and organizations: Software of the mind. New York, NY: McGraw-Hill.

Hofstede, G. (1998). Identifying organizational subcultures: An empirical approach. Journal of Management Studies, 35(1), 1-12.

Hofstede, G. (2001). Cultures consequences: Comparing values, behaviours, institutions, and organizations across nations. Thousand Oaks, CA: Sage.

Hofstede, G., \& Bond, M. (1988). The Confucius connection: From cultural roots to economic growth. Organizational Dynamics, 16(4), 5-22.

Hoppe, M. (1990). A comparative study of country elites: International differences in work-related values and learning and their implications for management training and development Unpublished PhD thesis, UNC at Chapel Hill, NC.

House of Commons Science and Technology Committee (2004). Scientific publications: Free for all? Retrieved 20 March 2006 from www.publications.parliament.uk/pa/cm200304/cmselect/cmsctech/399/399.pd f.

Huberman, A., \& Miles, M. (1998). Data management and analysis methods. In N. K. Denzin \& Y. S. Lincoln (Eds.), Collecting and interpreting qualitative materials (pp. 179-210). Thousand Oaks, CA: Sage Publications.

Huff, S., \& Munro, M. (1985). IT assessment and adoption: Field study. MIS Quarterly, 12, 327-338.

Huo, Y.P. \& Randall, D.M. (1991). Exploring subcultural differences in Hofstede's value survey: The case of the Chinese. Asia Pacific Journal of Management, 8(2), 159-173.

Huyer, S., \& Carr, M. (2002). Information and communication technologies: A priority for women. Gender, Technology and Development, 6(1), 85-100.

Huyer, S., \& Hafkin, N. (2007). Engendering the knowledge society: Measuring women's participation. Montreal, Canada: Orbicom, the International Network of UNESCO Chairs in Communication.

Huyer, S., Hafkin, N., Ertl, H., \& Dryburgh, H. (2005). Women in the information society. In G. Sciadas (Ed.), From the digital divide to digital opportunities: Measuring infostates for development. Montreal, Canada: Orbicom.

Huyer, S., \& Mitter, S. (2003). ICTs, globalization and poverty reduction: Gender dimensions of the information society. Retrieved 5 February 2009 from www.gab.wigsat.org

Huyer, S., \& Westholm, G. (2007). Gender indicators in science, engineering and technology: An information toolkit. Paris, France: UNESCO.

Iacovou, C., Benbasat, I., \& Dexter, A. (1995). EDI and small organizations: Adoption and impact of technology. MIS Quarterly, 12, 465-483.

Iivari, J. (1995). Factors affecting perceptions of CASE effectiveness. European Journal of European Systems, 4, 143-158. 
Inglehart, R. (1995). Changing values, economic development and political change. Internal Social Science Journal, 47, 379-404.

Inglehart, R., \& Baker, W. (2000). Modernization, cultural change and the persistence of traditional values. American Sociological Review, 65, 19-51.

Inglehart, R., Basanez, M., \& Moreno, A. (1998). Human values and beliefs: A crosssectional sourcebook; Findings from 1990-1993 world values survey: Ann Arbor, MI:The University of Michigan Press.

IT Governance Institute (2003). Board briefing on IT governance. Available from www.itgi.org and www.isaca.org

Ives, B. \& Jarvenpaa, S.L. (1991). Applications of global information technology: Key issues for management. MIS Quarterly, 15(1), 33-49.

Ives, B., Olson, M. H., \& Baroudi, J. J. (1993). The measurement of user information satisfaction. Communications of the ACM, 26(10), 785-793.

Juma, C. (2008a). G8 should support high-speed web access in Africa. Daily Yomiuri Online. Retrieved 13 June 2008, from www.scidev.net/en/science-andinnovation-policy/university-systems/opinions

Juma, C. (2008b). Net access for African universities would boost continent. Daily Yomiuri (May).

Juma, C. (Ed.). (2002). Going for growth: Science, technology and innovation in Africa. London, UK: The Smith Institute.

Kaduda, J., Olola, C. H. O., Oluoch, T. O., \& Mwazi, D. M. (2005). A digital library in a rural malaria research centre in Africa: the Kenyan experience. The International Information \& Library Review, 37, 3-10.

Kalambuka, A. H. (2008, May 13). Our parliament must change with times. Daily Nation, Retrieved 13 May 2008 from www.nationmedia.com/dailynation/printpage.asp

Kamau, N. (2001). Breaking information barriers: The Virtual Library Initiative $(A V L-I)$. Paper presented at The Web and Beyond: Harnessing the Potential of IT for Improving Health, Washington, DC, January 10.

Kaniki, A. (2004). Information Needs for Basic Research: An African Perspective. Paper presented at the Open Access and the Public Domain in Digital Data and Information for Science. Proceedings of an International Symposium, Paris.

Karahanna, E., Straub, D. W., \& Chervany, N. L. (1999). Information technology adoption across time: A cross-sectional comparison of pre-adoption and postadoption beliefs. MIS Quarterly 23(2), 183-213.

Karahanna, E., Evaristo, J. \& Srite, M. (2005). Levels of culture and individual behavior: An integrative perspective. Journal of Global Information Management, 13 (2), 1-20.

Kaye, G., \& Little, S. (2004). Dysfunctional development pathways of information and communication technology: Cultural conflicts. Journal of Global Information Management 8(2), 5-13.

Kebede, G. (2004). The information needs of end-users of Sub-Saharan Africa in the digital information environment. The International Information \& Library Review, 36, 273-279.

Kedia, B., \& Bhagat, R. (1988). Cultural constraints on technology transfer, etc. Academy of Management Review, 13, 559-571.

Keengwe, J., Kidd, T., \& Kyei-Blankson, L. (2009). Faculty and technology: Implications for faculty training and technology leadership. J Sci Educ Technol, 18, 23-28. 
Keil, M., Tan, B.C.Y., Wei, K.K., Saarinen, T., Tuuainen, V., \& Wassenaar, A. (2000). A cross-cultural study on escalation of commitment behavior in software projects. MIS Quarterly, 24(2), 299-325.

Kigotho, W. (2008, 30 September). Death of research in Africa. The Standard Retrieved 30 September 2008, from http://www.eastandard.net/

Kiiski, S., \& Pohjola, M. (2002). Cross-country diffusion of the internet. Information Economics and Policy, 14(2), 297-310.

Kiprotich, A., \& Otieno, S. (2008, 13 November). Universities are 'fixated with money'. The Standard. Retrieved 13 November 2008, from www.eastandard.net/

Kircz, J. G., \& Roosendaal, H. E. (1996). Understanding and shaping scientific information transfer Paper presented at the Electronic Publishing in Science Proceedings of the joint ICSU Press - UNESCO Expert Conference, Paris.

Kirkman, B.L., Lowe, K.B. \& Gibson, C.B. (2006). A quarter century of culture’s consequences: A review of empirical research incorporating Hofstede's cultural values framework. Journal of International Business Studies, 37, 285320.

Kirsop, B. (2002). Electronic Publishing Trust for Development. Bangalore electronic publishing workshop, 27 March 2002. Retrieved 20 April 2006, from www.ias.ac.in/epubworkshop/presentations/epub_DCs/sld007.html

Klein, H., \& Myers, M. (1999). A set of principles for conducting and evaluating interpretive field studies in Information Systems. MIS Quarterly, 23(1), 67-94.

Kling, R. (1999). What is social informatics and why does it matter? D-Lib Magazine, 5(1). Retrieved 25 June 2006 from www.dlib.org.80/dlib/january99/kling/01kling.html

Kling, R. (2004). The Internet and unrefereed scholarly publishing. In B. Cronin (Ed.), Annual review of information science and technology (Vol. 38, pp. 591631). Medford, NJ: Information Today.

Kling, R., \& Callahan, E. (2002). Electronic journals, the Internet, and scholarly communication. CSI Working papers, No. 01-04 (Draft 6.03) Retrieved 28 June 2006 from www.slis.indiana.edu./csi/WP/wp01-04B.html

Kling, R., \& McKim, G. (1999). Scholarly communication and continuum of electronic publishing. Journal of the American Society for Information Science and Technology 50(9), 890-906. Retrieved 28 June 2006 from www.xxx.lanl.gov/ftp/cs/papers/9903/9903015.pdf

Kling, R., McKim, G., \& King, A. (2003). A bit more to it: Scholarly communication forums as socio-technical interaction networks. Journal of the American Society for Information Science and Technology, 54(1), 47-67.

Kogut, B., \& Singh, H. (1988). The effect of national culture on the choice of entry mode. JIBS(Fall), 411-432.

Korpela, M. (1996). The traditional culture or political economy? On the root causes of organizational obstacles of IT in developing countries. Information Technology for Development, 7, 29-42.

Kumar, A. (2001). Bridging the digital divide--some efforts from Keral. Retrieved 8 October 2008: www.unpan1.un.org/intradoc/groups/public/documents/apcity/unpan005827.p df

Kumar, V., Ganesh, J., \& Echambadi (1998). Cross-national diffusion research: What do we know and how certain are we? Journal of Product Innovation Management, 15, 255-268. 
Kwon, T., \& Zmud, R. (1987). Unifying the fragmented models of IS implementation. In R. Boland \& H. R (Eds.), Critical issues in IS research (pp. 227-251): Stafford, QLD: John Wiley.

Lal, K. (2001). Institutional environment and the growth of Indian IT industry. The Information Society, 17, 105-117.

Latour, B. (1988). Drawing Things Together. In M. Lynch \& S. Woolgar (Eds.), Representation in scientific practice (pp. 19-68). Cambridge, MA: MIT Press.

Lee, M., Davidson, R., \& Wei, K. K. (1999). The adoption and diffusion of collaborative systems and technology. Paper presented at The 32nd Hawaii International Conference on System Sciences (HICSS), Hawaii.

Lee, M. K. O., \& Cheung, C. M. K. (2004). Internet retailing adoption by small-tomedium sized enterprises (SMEs): A multiple-case study Information Systems Frontiers, 6(4), 385-397.

Lefevre, E., \& Lefevre, L. (1996). Information and telecommunications technologies: Ottawa, Canada: IDRC Press.

Leidner, D. \& Kayworth, T. (2006). A review of culture in information systems research: Toward a theory of information technology culture conflict. MIS Quarterly, 30(2), 357-399.

Limo, A. (2008a, 6 July). Digital World - Technology now shifts focus from computing to communication Daily Nation, Retrieved 6 July 2008 from www.nationmedia.com/dailynation/

Limo, A. (2008b, 26 January). How computer can be made an effective classroom tool Daily Nation. Retrieved 26 January 2008, from www.nationmedia.com/dailynation/nmgcontententry.asp

Limo, A. (2008c, 19 July). Parents must act now to control cyber culture among children. Daily Nation, Retrieved 19 July 2008 from www.nationmedia.com/dailynation/nmgcontententry.asp

Limo, L. (2008, 14 May). Produce drugs locally, Nyong'o tells Kemri. The Standard. Retrieved May 214, 2008, from www.eastandard.net/print.php

Lincoln, Y. S., \& Guba, E. G. (1985). Naturalistic inquiry. Beverly Hills, CA: Sage Publications.

Livingstone, D. N. (2003). Putting science in its Place: Geographies of scientific knowledge. Chicago, IL: University of Chicago Press.

Longino, H. (2002). The fate of knowledge. Princeton, NJ: Princeton University Press.

Makhubela, L. (2004). Introductory remarks by session chair. Paper presented at the Open Access and the Public Domain in Digital Data and Information for Science. Proceedings of an International Symposium, Paris.

Mansell, R., \& Wehn, U. (Eds.). (1998). Knowledge societies. Oxford, UK: Oxford University Press.

Marcus, A., \& Gould, E. W. (2000). Cultural dimensions and global web userinterface design: What? So what? Now what? Paper presented at The 6th Conference on Human Factors and the Web, 19 June 2000, Austin, Texas.

Mason, J. (1996). Qualitative researching. London, UK: Sage.

Matsuura, K. (2004). UNESCO's approach to open-access and public-domain information. Paper presented at the Open Access and the Public Domain in Digital Data and Information for Science. Proceedings of an International Symposium, Paris.

Mazrui, A. (1978). The African university as a multi-national corporation. In A. Mazrui (Ed.), Political values and the educated class in Africa (pp. 285-319). London, UK: Heinemann. 
Mbarika, V., Jensen, M., \& Meso, P. (2002). International perspectives: Cyberspace across Sub-Saharan Africa. Communications of the ACM, 45(12), 17-21.

McCoy, S. (2003). Integrating national culture into individual IS adoption research: The need for individual level measures. Proceedings of the Ninth American Conference on information systems (AMCIS) 2003, Tampa, Florida, USA [CD].

McSweeney, B. (2002). Hofstede's model of national cultural differences and their consequences: A triumph of faith - a failure of analysis. Human Relations, 55(1), 89-118.

Meadows, A. J. (1998). Communicating research. London: Academic Press.

Mejias, R. (1995). A cross-cultural comparison of GSS outcomes: A US and Mexico field equipment. Unpublished PhD thesis, The University of Arizona.

Menon, M. (2004). Introduction by Symposium Chair. Paper presented at the Open Access and the Public Domain in Digital Data and Information for Science. Proceedings of an International Symposium, Paris.

Milberg, S., Burke, S., Smith, H. J., \& Kallman, E. (1995). Values, personal information, privacy and regulatory approaches. Communications of the ACM, 12, 65-74.

Miles, M. B., \& Huberman, A. M. (1994). Qualitative data analysis: An expanded sourcebook. London, UK: Sage.

Moon, M. (2000). Organizational commitment revisited in new public management: Motivation, organizational culture, sector, and managerial level. Public Performance \& Management Review 24 (2), 177-94.

Moon, M. \& Bretschneider, S. (2002). Does the perception of red tape constrain IT innovativeness in organizations? Unexpected results from a simultaneous equation model and implications. Journal of Public Administration Research and Theory 12 (2), 273-91.

Moore, G., \& Benbasat, I. (1991). Adoption of IT innovation. Information Systems Research, 9, 192-222.

Moore, G. C., \& Benbasat, I. (1996). Integrating diffusion of innovations and theory of reasoned action models to predict utilization of information technology by end-users. In K. Kautz \& J. Pries-Hege (Eds.), Diffusion and Adoption of Information Technology (pp. 132-146). London, UK: Chapman and Hall.

Morales-Gomez, D., \& Melesse, M. (1998). Utilizing information and communication technologies for development: The social dimensions. Information Technology for Development, 8(1), 3-13.

Morton, B. (1997). Is the journal as we know it an article of faith? An open letter to the faculty. The Public-Access Computer Systems Review 8(2). Retrieved 25 April 2006 from www.info.lib.uh.edu/pr/v8/n2/mort8n2.html

Muinde, F. (2004). The Public knowledge Project's open journal systems. Paper presented at the Open Access and the Public Domain in Digital Data and Information for Science. Proceedings of an International Symposium, Paris.

Munoz, G. (2006). Local scientific journals. Are local journals important? An indebate in INASP discussion group. Retrieved 2 August 2006, from www.dgroups.org/groups/IN-debate

Musa, P. F., Mbarika, V. W., \& Meso, P. (2005). Calling for programmed technology transfer and adoption strategies for sustainable LDC growth. Communications of the ACM, 48(12), 111-116.

Muthayan, S. (2004). Open-access research and the public domain in South African universities: The public knowledge project's open journal systems. Paper 
presented at the Open access and the public domain in digital data and information for science: Proceedings of an international symposium, Paris.

Mutula, S. M. (2001a). Internet access in East Africa: A future outlook. Library Review, 50(1), 28-33.

Mutula, S. M. (2001b). The IT environment in Kenya: Implications in public universities. Library Hi Tech, 19(2), 155-166.

Mwaura, P. (2008, April 19 ). Kenya is still sleeping on its intellectual property rights. Daily Nation Retrieved April 19 2008, from www.nationamedia.com/dailynation/printpage.asp

Mwesige, P. G. (2003). Cyber elites: A survey of internet cafe users in Uganda. Telematics \& Informatics, 21, 83-101.

Myers, M. (1997). Qualitative research in Information Systems. MIS Quarterly, 21(2), 241-242. Retrieved 20 October 2006 from www.misq.org/discovery/MISQD_isworld/index.html .

Myers, M. \& Tan, F. (2002). Beyond models of national culture in information systems research. Journal of Global Information Management 10(1), 24-32.

Nakata, C., \& Shivakumar, K. (1996). National culture and new product development: An integrative review. Journal of Marketing, 1, 61-72.

Nath, R. \& Murphy, N. (2004). A study of the relationship between Internet diffusion and culture. Journal of International Technology and Information Management 13 (2), 123-132.

Ndede-Amadi, A. (2006). National digital indicators and e-government models: A status assessment for Kenya. In G. Outa, F. Etta \& E. Aligula (Eds.), Mainstreaming ICT: Research perspectives from Kenya (pp. 117-168). Nairobi: MvuleAfrica Publishers.

Nduati, C., \& Bowman, W. (2005). Working from the sidelines: The Kenyan Private Sector Foundation. In F. Etta \& L. Elder (Eds.), At the crossroads: ICT policy making in East Africa (pp. 56-67). Nairobi, Kenya: East African Educational Publishers Ltd/International Development Research Centre, Canada.

Nentwich, M. (2003). Cyberscience: Research in the age of the Internet. Vienna, Austria: Austrian Academy of Science Press.

Nentwich, M. (2005). Cyberscience: Modelling ICT- induced changes of the scholarly communication. Information, Communication \& Society, 8(4), 542-560.

Newell, S., Swan, J. A., \& Galliers, R. D. (2000). A knowledge-focused perspective on the diffusion and adoption of complex information technologies: the BPR example. Info Systems Journal, 10, 239-259.

NIH (2003). Policy on enhancing public access to archived publications resulting from NIH-funded research. Notice Number: NOT-OD-05-022 February 3 2003. Retrieved 28 February 2006, from www.grants2.nih.gov/grants/guide/notice-files/NOT-OD-05-022.htm

NIH (2008). Revised policy on enhancing public access to archived publications resulting from $\mathrm{NIH}$-funded research. Retrieved 30 June, 2008, from www.grants.nih.gov/grants/guide/notice-files/NOT-OD-08-033.html

NIH (2009). The Omnibus Appropriations Act of 2009 makes the NIH public access policy permanent. Retrieved 30 April 2009, from www.grants.nih.gov/grants/guide/notice-files/NOT-OD-09-071.html

Nilakanta, S., \& Scamell, R. (1990). The effects of information sources and communication channels on the diffusion of innovation in a data base development environment. Management Science, 36, 24-40. 
Nystrom, P, K Ramamurthy \& Wilson, A. (2002). Organizational context, climate and innovativeness: adoption of imaging technology. Journal of Engineering Technology Management 19, 221-47.

O'Callaghan, R., Kaufmann, P., \& Konsynski, B. (1992). Adoption correlates and share effects of EDI systems in marketing channels. Journal of Marketing, 56, 45-56.

Ochuodho, S., \& Matunga, M. (2005). A national orchestra? Civil society involvement in ICT policy making. In F. E. Etta \& L. Elder (Eds.), At the crossroads: ICT policy making in East Africa (pp. 68-83). Nairobi, Kenya: East African Educational Publishers Ltd/International Development Research Centre.

Odero-Musakali, D., \& Mutula, S. (2007). Internet adoption and assimilation in Kenyan university libraries. Library Review, 56(6), 464-474.

OECD (1998). Science, technology and industry outlook 1998 - Chapter 7: The global research village: How information and communication technologies affect the science system. Retrieved 2 June 2006 from www.oecd.org/pdf/M000014000/M00014833.pdf

OECD (2004). Science, technology and Innovation for the 21st Century. Meeting of the OECD Committee for Scientific and Technological Policy at Ministerial Level, 29-30 January 2004 - Final Communiqué. Retrieved 2 June 2006, from www.oecd.org/documentprint/0,2744,en_2649_34487_25998799_1_1_1_1,00 .html

Olson, G. M. (2005). Long-distance collaborations in science: Challenges and opportunities. Keynote talk presented at the First International Conference on e-Social Science, Manchester, UK.

Olson, G. M., \& Olson, J. S. (2000). Distance matters. Human-Computer Interaction, 15(2-3), 139-178.

Ondari-Okemwa, E. (2002). Challenges of harnessing virtual information resources in Kenya: The case of the African virtual university. Journal of Information Science, 28(4), 321-329.

Onu, T. (2008, 24-27 August). Only connect? 3 key issues on ICTs and farmers. Paper presented at the World Conference on Agricultural information and IT, Tokyo, Japan.

Orlikowski, W., \& Baroudi, J. (1991). Studying information technology in organizations: Research approaches and assumptions. Information Systems research, 2(1), 1-28.

Osanjo, T. (2009). Free access to journals gives Kenya science a boost. Retrieved 5 February 2009 from www.who.int/bulletin/volumes/84/9/06030906/en/print.html

Ouya, D. (2006). Open Access survey of Africa-published journals. INASP Infobrief 7(June). Retrieved 20 September 2006 from www.inasp.info

Oyelaran-Oyeyinka, B., \& Adeya, C. N. (2002). Determinants of academic use of the Internet in Nigerian universities. Unpublished UNU/INTECH Discussion Paper DP-2002-5. United Nations University.

Oyelaran-Oyeyinka, B., \& Lal, K. (2005). Internet diffusion in sub-Saharan Africa: A cross-country analysis. Telecommunications Policy, 29, 507-527.

Oyomno, G. Z. (2006). National digital indicators and e-government models for Africa: A conceptual and theoretical foundation. In G. Outa, F. Etta \& E. Aligula (Eds.), Mainstreaming ICT: Research perspectives from Kenya (pp. 73-116). Nairobi, Kenya: MvuleAfrica publishers. 
Pehrson, B. (2005). Open communication - Open access. In S. Danofsky (Ed.), Open access for Africa: Challenges, recommendations and examples (pp. 5-15). New York, NY: United Nations ICT Task Force.

Pettigrew, A.M. (1979). On studying organizational culture. Administrative Science Quarterly, 24(4), 570-581.

Plouffe, C. R., Hulland, J. S., \& Vandenbosch, M. (2001). Research report: Richness versus parsimony in modeling technology adoption decisionsUnderstanding merchant adoption of a smart card-based payment system. Information Systems Research, 12(2), 208-222.

Plouffe, C. R., Vandenbosch, M., \& Hulland, J. (2001). Intermediating technologies and multi-group adoption: A comparison of consumer and merchant adoption intentions toward a new electronic payment system. Journal of Product Innovation Management, 18(2), 65-81.

Png, I. P. L., Tan, B. C. Y., \& Wee, K. L. (2001). Dimensions of national culture and corporate adoption of it infrastructure. IEEE transactions on Engineering Management, 48(1), 36-45.

Powell, R. R., \& Connaway, L. S. (2004). Basic research methods for librarians (4 ed.). Westport, CN: Libraries Unlimited.

Premkumar, G., \& Potter, M. (1995). Adoption of CASE technology: An innovation adoption perspective. Data Base Advances, 26, 105-123.

Premkumar, G., Ramamurthy, K., \& Nilakanta, S. (1994). Implementation of EDI: An innovation diffusion perspective. Journal of MIS, 11, 157-186.

Prescott, M., \& Conger, S. (1995). IT innovations: A classification by IT locus of impact and research approach. Data Base Advances (May/August), 20-40.

Press, L., Burkhart, G., Foster, W., \& Goodman, S. E. (1998). An Internet diffusion Framework. Communications of the ACM, 41, 21-26.

QSR (2003). Overview: Qualitative research software solutions to meet every need. Retrieved 3 June 2006, from www.qsrinternational.com

Rai, A., Ravichandran, T., \& Samaddar, S. (1998). How to anticipate the Internet's global diffusion. Communications of the ACM, 41, 97-106.

Ratsatsi, D. M. (2002). Botswana: Equity and access in ICTs. TechKnowLogia. Retrieved 18 February 2007 from www.TechKnowlogia.org

Reardon \& Rogers, E.M. (1988). Interpersonal versus mass media communication: A false dichotomy. Human Communication Research 15(2), 284-303.

Republic of Kenya (1988). The Presidential working party to review the quality and relevance of education for the next decade and beyond. Nairobi, Kenya: Government Printer.

Rodriguez, F., \& Wilson, I. E. J. (2000). Are poor countries losing the information revolution? InfoDEV Working Paper Series. Retrieved 2 May, 2006, from www.cidcm.umd.edu/library/papers/ewilson/apxc.pdf 2

Rogers, E. M. \& Shoemaker, F. (1971).Communication of innovations: A crosscultural approach: NY: Free Press.

Rogers, E. M. (1976). New product adoption and diffusion. Journal of Consumer Research 2.

Rogers, E. M. (1986). Communication technology: The new media in society: NY: Free Press.

Rogers, E. M. (1995). The Diffusion of innovation (4th. ed.). New York, NY: Free Press.

Rogers, E. M., Takegami, S. \& Yin, J. (2001). Lessons learnt about technology transfer. Technovation 21(4), 253-261. 
Rogers, E. M. (2003). Diffusion of innovations. (5th ed.). New York, NY: Free Press. Rogers, E. M. (2004). A prospective and retrospective look at the diffusion model. Journal of Health Communication: International Perspective, 9(6) Supplement 1, 13-19.

Rogers, E. M., \& Shoemaker, F. (1971). Communications of innovations. New York, NY: Free Press.

Roosendaal, H., \& Geurts, P. (1997). Forces and functions in scientific communication: An analysis of their interplay Paper presented at the Cooperative Research Information Systems in Physics, August 31September 4 1997, Oldenburg, Germany.

Rosenberg, D. (1998). IT and universities libraries in Africa. Internet Research: Electronic Networking Applications and Policy, 8(1), 5-13.

Rosenberg, D. (2005). The current digital status of university libraries in Africa. INASP Newsletter, 30(Autumn ). Retrieved 10 March 2006 from www.inasp.info/newslet/nov05.shtml

Sahu, D. K. (2006). Open access in the developing world: Regaining the lost impact. Workshop on electronic publishing and open access, 2-3 November 2006 Retrieved 1 April 2007, from www.epublishingtrust.org Sahu, D. K., \& Chan, L. (2004). Bioline International and the Journal of postgraduate medicine: A collaborative model of open-access publishing. Paper presented at the Open Access and the Public Domain in Digital Data and Information for Science. Proceedings of an International Symposium, Paris.

Schrum, L., Skeele, R., \& Grant, M. (2002). One college of education's effort to infuse technology: A systemic approach to revisioning teaching and learning. J Res Technol Educ, 35(2), 256-271.

Schwarz, S., \& Bilsky, W. (1990). Toward a theory of the universal content and structure of values: Extensions and cross-cultural replications. Journal of Personality and Social Psychology, 58, 878-891.

Schwarz, S., \& Sagiv, L. (1995). Identifying culture-specifics in the content and structure of values. Journal of Cross-Cultural Psychology, 26, 92-116.

Segars, A., Grover, V., \& Durand, D. (1994). Cultural diversity in organizational practice, information resource deployment and system success: An analysis of French, Korean and US firms. Journal of Strategic Information Systems, 13(2), 85-106.

Sein, M. K., \& Harindranath, G. (2004). Conceptualizing the ICT artifact: Towards understanding the role of ICT in national development. The Information Society, 20, 15-24.

Shibanda, G. (2006). Enhancing e-journal use in Kenyan universities. Paper presented at the CODESRIA ASC Conference on Electronic Publishing and Dissemination 6-8 September 2006.

Sihanya, B., \& Odek, J. O. (2006). Regulating and mainstreaming ICT for Kenya's socio-economic development. In G. Outa, F. Etta \& E. Aligula (Eds.), Mainstreaming ICT: Research perspectives from Kenya (pp. 169-224). Nairobi, Kenya: MvuleAfrica publishers.

Silverman, D. (2001). Research and social theory. In C. Seale (Ed.), Researching society and culture (2 ed., pp. 97-110). London, UK: Sage.

Simborg, D. W. (2008). Promoting electronic health record adoption. Is it the correct focus? Journal of the American Medical Informatics Association, 15(2), 127129 
Smart, P. (2003). Supporting indigenous published research: The African Journals Online (AJOL) case study. Paper presented at the ELPUB2003: From information to knowledge, June 25-28, Guimaraes, Portugal.

Smart, P. (2005). African Journals OnLine (AJOL). Serials Review, 31, 261-265.

Smith, P., Dugan, S., \& Trompenaars, F. (1996). National culture and values of organizational employees. Journal of Cross-Cultural Psychology, 27, 231264.

Sondergaard, M. (1994). Hofstede's consequences: A study of reviews, citations and replications. Organization Studies, 15, 447-456.

Sonnenwald, D. H. (2007). Scientific Collaboration. Annual Review of Information Science and Technology, 41, 643-681.

Steenkamp, J.-B., Hofstede, F., \& Wedel, M. (1999). A cross-national investigation into the individual and national cultural antecedents of consumer innovativeness. Journal of Marketing, 63(April), 55-69.

Stepulevage, L. (2001). Gender/technology relations: complicating the gender binary. Gender and Education, 13(3), 325-338.

Strang, D., \& Soule, S. A. (1998). Diffusion in organisations and social movements: From hybrid corn to poison pills. Annual Review of Sociology, 24, 265-290.

Straub, D. W., Keil, M., \& Brenner, W. (1997). Testing the technology acceptance model across cultures: A three country study. Information \& Management Journal, 33(1), 1-11.

Straub, D. W. (1994). The effect of culture on IT diffusion: E-mail and fax in Japan and U.S. Information Systems Research, 5(1), 23-47.

Straub, D. W., Loch, K., Evaristo, R., Karahanna, E. \& Srite, M. (2002). Toward a theory-based measurement of culture. Journal of Global Information Management, 10(1), 13-23.

Straub, D. W., Hill, C., Loch, K., \& El-Sheshai, K. (1998). A qualitative assessment of Arab culture and information technology transfer. Journal of Global Information Management, 6(3), 29-38.

Strauss, A., \& Corbin, J. (1990). Basics of qualitative research. Newbury Park, CA: Sage publications.

Swanson, E. (1994). Information systems innovation among organizations. Management Science, 1069-1094.

Tan, B.C.Y. Wei, K.K., Watson, R.T. \& Walczuch, R.M. (1998). Reducing status effects with computer-mediated communication: Evidence from two distinct national cultures. Journal of Management Information Systems 15(1), 119141.

Tayeb, M. (1994). Organizations and national culture: Methodology considered. Organization Studies, 15(3), 429-446.

Taylor, S., \& Todd, P. A. (1995a). Assessing IT usage: The role of prior experience. MIS Quarterly, 19(2), 561-570.

Taylor, S., \& Todd, P. A. (1995b). Understanding information technology usage: A test of competing models. Information Systems Research, 6(4), 144-176.

Teferra, D. (1998). The significance of information technology for African scholarly journals. In P. G. Altbach \& D. Teferra (Eds.), Knowledge dissemination in Africa: The role of scholarly journals (pp. 39-62). Chestnut Hill, MA: Bellagio Publishing Network,.

Teferra, D. (2003). Scientific communication in African universities: External assistance and national needs. New York: RoutledgeFalmer. 
Tetiwat, O. (2003). A cross cultural study of factors influencing the adoption and diffusion of web-based educational technology: Comparative study of New Zealand and Thai tertiary educators Unpublished PhD dissertation, Victoria University of Wellington, Wellington.

Thompson, J. B. (2005). Books in the digital age: The transformation of academic and higher education publishing in Britain and the United States. Cambridge, UK: Polity Press.

Thompson, R. L., Higgins, C. A., \& Howell, J. M. (1991). Personal computing: Toward a conceptual model of utilization. MIS Quarterly, 15(1), 124-143.

Tornatzky, L. G., \& Klein, K. J. (1982). Innovation characteristics and innovation adoption-implementation: A meta-analysis of findings. IEEE Transactions on Engineering Management, EM-29(1), 28-45.

Tran, L. A. (2006). Public libraries and community information networks in New Zealand: An assessment and evaluation of practices. Unpublished $\mathrm{PhD}$ dissertation, Victoria University of Wellington, Wellington.

Triandis, H.C. (1995). Individualism \& collectivism. Boulder, CO: Westview Press.

Triandis, H. C. (1977). Interpersonal behaviour. Monterey, CA: Brook/Cole.

Triandis, H.C. (1994). Theoretical and methodological approaches to the study of collectivism and individualism. In: U Kim, H.C. Triandis, C. Kagitcibasi, S-C. Choi \& G. Yoon (Eds), Individualism and collectivism: Theory, methods and applications (pp. 41-51). London, UK: Sage.

Trompenaars, A., \& Hamden-Turner, C. (1993). Riding the waves of culture: Understanding cultural diversity in global business. London, UK: Brealey.

UNECA (1996). Prospects for I.T in Africa. Paper presented at the 9th Session of the Conference of African Planners, Statisticians, Population and Information Specialists, Addis Ababa, Ethiopia.

UNESCO (2002). International literacy day 2002. Regional illiteracy statistics. Retrieved 10 October 2006 from www.uis.unesco.org/en/stats/statistics/literacy2000.htm

UNESCO (2005). Towards knowledge societies. Paris, France: UNESCO.

UNESCO (2009). East/North Africa regional science technology and innovation policy reviews workshop 30 March-3 April 2009 Retrieved 5 April 2009, from UNESCO: www.unesco.org/science/psd/thm_innov/workshop_eastnorth_africa.pdf

Vallerand, R. J. (1997). Towards a hierarchical model of intrinsic and extrinsic motivation. In M. Zanna (Ed.), Advances in experimental social psychology (Vol. 29, pp. 271-360). New York: Academic Press.

Van de Sompel, H., Payette, S., Erickson, J., Lagoze, C., \& Warner, S. (2004). Rethinking scholarly communication: Building the system that scholars deserve. D-Lib Magazine, 10(9). Retrieved 20 March 2006 from www.dlib.org/dlib/september04/vandesompel/09vandesompel.html\#wate $\underline{\text { rS }}$

Van de Ven, A. \& Rogers, E. (1988). Innovations and organizations. Communication Research 15, 632-51.

Vandooren, F. (2002). The future of scholarly communication: Conclusions and recommendations. Paper presented at The Future of Scholarly communication, UNICA Seminar 24-25 October 2002, Madrid.

Venkatesh, V., \& Davis, F. D. (2000). A theoretical extension of the technology acceptance model: Four longitudinal field studies. Management Science, 45(2), 186-204. 
Venkatesh, V., \& Morris, M. G. (2000). Why don’t men ever stop to ask for directions? Gender, social influence, and their role in technology acceptance and usage behavior. MIS Quarterly 24(1), 115-139.

Venkatesh, V., Morris, M. G., Davis, G. B., \& Davis, F. D. (2003). User acceptance of information technology: Toward a unified view. MIS Quarterly, 27(3), 425478.

Venkatesh, V., \& Speier, C. (1999). Computer technology training in the workplace: A longitudinal investigation of the effect of the mood. Organizational Behavior and Human Decision Processes 79(1), 1-28.

Ventola, E. (2007). Writing scientific English: Overcoming intercultural problems. International Journal of Applied Linguistics, 2(2), 191-220.

Volman, M., \& van ECK, E. (2002). Gender equity and information technology in education: The second decade. Journal of Educational Research, 71(4).

Wafula, J. M., \& Etta, F. (2006). An overview of ICT initiatives in Kenya. In G. Outa, F. Etta \& E. Aligula (Eds.), Mainstreaming ICT: Research perspectives from Kenya (pp. 7-24). Nairobi, Kenya: MvuleAfrica publishers.

Walsham, G. (2002). Cross-cultural software production and use: A structural analysis. MIS Quarterly, 26(4), 359-380.

Walsham, G. (1993). Interpreting information systems in organizations. Chichester, UK: Wiley.

Walsham, G. (1995). Interpretive case studies in IS research: Nature and method. European Journal of Information Systems, 4(2), 74-81.

Warner, S. (2005). The transformation of the scholarly communication. Learned Publishing, 18(3), 177- 185.

Weisinger, J.Y. \& Trauth, E.M. (2003). The importance of situating culture in crosscultural IT management. IEEE Transactions on Engineering Management, 50(1), 26-30.

Willinsky, J. (2006). The access principle: The case for open access to research and scholarship. Boston, MA: Massachusetts Institute of Technology Press.

Winch, G., Millar, C. \& Clifton, N. (1997). Culture and organization: The case of Transmanche-Link. British Journal of Management 8(3), 237-249.

World Bank (1998/1999). World Bank world development report: Knowledge for development. Washington, D.C: World Bank Institute.

World Summit on the Information Society (2003). Declaration principles building the information society: A global challenge in the new millennium. World summit on the information society. Retrieved 1 March 2006, from www.itu.int/dms_pub/itu-s/md/03/wsis/doc/S03-WSIS-DOC-0004!!PDFE.pdf

Young, K. S. (1998). Caught in the net: How to recognize the signs of Internet addiction and $a$ winning strategy for recovery. Available from 20 August 2008 www.books.google.co.nz

Zeleza, P. T. (1998). The challenges of editing scholarly journals in Africa. In P. G. Altbach \& D. Teferra (Eds.), Knowledge dissemination in Africa: The role of scholarly journals (pp. 13-38). Chestnut Hill, MA: Bellagio Publishing Network,.

Zell, H. M. (1992). African publishing: Constraints and the experience of African Books Collective (ABC). In P. G. Altbach (Ed.), Publishing and development in the Third World (pp. $101-118$ ). London,UK: Hans Zell Publishers. 
Zhang, Y. (1998). The impact of Internet-based electronic resources on formal scholarly communication in the area of library and information science: A citation analysis. Journal of Information Science 24(4), 241-254.

Zhong (1993). China's participation in the world economy: A study of Chinese scholarly communication. University of Toronto, Toronto.

Zmud, R. W. (1982). Diffusion of modern S/W practices. Management Science, 28, 1421-1431.

Zmud, R. W. (1983). The effectiveness of external information channels in facilitating innovation within S/W development group. MIS Quarterly (June), 43-58. 


\section{APPENDICES}

\section{A-1: Timeline for the PhD. study}

\begin{tabular}{|c|c|}
\hline $\begin{array}{l}\text { March 1, 2006- } \\
\text { February 28, } 2007\end{array}$ & - $\quad$ Develop and present proposal \\
\hline $\begin{array}{l}\text { March -April 15, } \\
2007\end{array}$ & $\begin{array}{ll}\text { - } & \text { Arrive in Kenya } \\
\text { - } & \text { Apply for authority to undertake the research from the Ministry } \\
& \text { of Science \& Technology } \\
\text { - } & \text { Pilot study in } 1 \text { research institute and analyse the data } \\
\text { - } & \text { Review interview guidelines following the pilot } \\
\text { - } & \text { Send to supervisors for consensus building } \\
\text { - } & \text { Factor in adjustments by supervisors. }\end{array}$ \\
\hline $\begin{array}{l}\text { April 16- May 15, } \\
2007\end{array}$ & $\begin{array}{l}\text { - Interview university/research institutes administrators; faculty } \\
\text { deans; transcribe the data and send it back to them for validation } \\
\text { - } \quad \text { Interview researchers in the selected } 2 \text { universities; transcribe the } \\
\text { data and take it back to them for validation } \\
\text { - Interview head librarians in the } 2 \text { universities; transcribe the data } \\
\text { and send it back to them for validation } \\
\text { - Collect any relevant documentary evidence like policy } \\
\text { documents, annual and technical reports, strategic plans, etc. and } \\
\text { review them. }\end{array}$ \\
\hline $\begin{array}{l}\text { May 16- June 30, } \\
2007\end{array}$ & $\begin{array}{ll}\text { - } & \text { Interview researchers from the selected } 6 \text { research } \\
\text { - } & \text { Transcribe interviews and send back to researchers for validation } \\
\text { - } & \text { Interview librarians in the research institutes; transcribe data } \\
\text { from librarians and send it back to them for validation } \\
\text { - } \quad \text { Collect any relevant documentary evidence like policy } \\
\text { documents, annual and technical reports, strategic plans, etc. and } \\
\text { review them. }\end{array}$ \\
\hline $\begin{array}{l}\text { July } 1 \text {-July 20, } \\
2007\end{array}$ & $\begin{array}{l}\text { Interview journal editors in the relevant disciplines in applied sciences } \\
\text { and technology; transcribe the data and send it back to them for } \\
\text { validation. }\end{array}$ \\
\hline $\begin{array}{l}\text { July } 21 \text {-August 21, } \\
2007\end{array}$ & $\begin{array}{l}\text { - Interview representatives from relevant government } \\
\text { Ministries/departments; transcribe the data and send it back to } \\
\text { them for validation } \\
\text { - Collect any relevant documentary evidence like policy } \\
\text { documents, annual and technical reports, etc. and review them. }\end{array}$ \\
\hline August 22-30, 2007 & $\begin{array}{l}\text { - Travel back to New Zealand to finalise data analysis and thesis } \\
\text { writing. }\end{array}$ \\
\hline $\begin{array}{l}\text { September 2007- } \\
\text { April } 2008\end{array}$ & Data analysis and synthesis \\
\hline $\begin{array}{l}\text { May } 2008 \text { - May } \\
\text { 30, } 2009\end{array}$ & Thesis writing and editing \\
\hline June 2009 & Thesis finalisation and submission \\
\hline
\end{tabular}




\title{
A-2: A letter of introduction to the heads of the research institutions
}

\author{
Dear Sir/Madam,
}

\section{RE: AUTHORITY TO UNDERTAKE RESEARCH IN YOUR INSTITUTION}

I am a PhD student at Victoria University of Wellington (VUW), New Zealand, in the School of Information Management (SIM) and I have selected your institution to participate in my research. The project title is: "An investigation of factors affecting adoption of ICT for communication of research output by researchers in research institutions in Kenya."

My study seeks to contribute to the understanding of ICT-enabled research dissemination by and for scholars and researchers working in Kenya, the main purpose of the study being to identify factors affecting ICT-enabled research dissemination; the communication needs and priorities for researchers in Kenya. This will be done by developing, assessing and then revising a contextual model for ICT-enabled research dissemination for Kenya-based researchers in specific fields within the applied sciences. The model will be used in identifying, explaining and prioritizing key factors that will assist the Kenyan research community in more effectively adopting ICT-enabled research dissemination practices.

Data will be gathered by interviewing researchers employed in a range of institutions based in Kenya, including universities, government and international research institutes; research institutions' administrators; representatives from relevant government Ministries/departments; members of research councils and research sponsors; research librarians and journal editors. The interviews will deal with general questions about ICT adoption in relation to communication of research output in your institution. The insights gained will be used as part of my $\mathrm{PhD}$ thesis, which will be deposited in the VUW library on completion. In addition, aggregate data may be published in case studies, academic or professional journals and presented at conferences.

The project has been approved by the VUW SIM Human Ethics Committee. All raw data will be kept confidential to the researcher and the two supervisors: Prof. Gary Gorman and Dr. Chern Li Liew. Throughout the project, raw data will be kept under password and/or lock protection and destroyed two years after the conclusion of the project. Any information and opinions gathered from members of your institute will be attributed neither to the institute nor its members; data will be presented only in aggregate form. You are free to withdraw from the study without any explanation any time before 30 June 2007. In case you opt to withdraw, all the information provided will be withdrawn from the study. A summary of the key findings and a research report will be provided to you and all other research participants upon request.

The purpose of this letter is, therefore, to seek your authority to carry out this research in your institution.

The consent form is attached. It includes a request for permission to tape-record interviews. If you agree to participate, please complete the form, sign and return it to me.

Thank you for time and cooperation.

Florence N.N. Muinde

Phone: +254-733-881841/+254-20-227411

Email: Florence.Muinde@vuw.ac.nz 


\section{A-3: Current Situation of ICT Infrastructure in Kenya}

Kenya has witnessed significant growth in the ICT sector as demonstrated by the number of telephone lines, Internet Service Providers (ISPs), Internet users and broadcasting stations, and the market share of each. The Government has also liberalised the mobile cellular communication market and there are currently two mobile cellular operators.

A government report (Government of Kenya 2006) showed there were:

- 260,000 fixed telephone line subscribers and 3.0 million cellular mobile subscribers by June 2004, translating into fixed teledensity of 0.75 per hundred inhabitants for fixed and 9.75 per hundred inhabitants for mobile against the world average of 19 and 21 respectively

- 73 registered ISPs, 16 of which are active, approximately 1,030,000 users and over 1,000 cyber cafes and telephone bureaus by June 2005

- 16 operational television stations and 24 FM radio stations

- Approximately 11,500 public phones installed throughout the country by the year 2003

- An estimated $60 \%$ of the population with access to television and $90 \%$ with access to radio services ( p.694). In addition, the following has been achieved:

- Websites have been developed in government ministries/departments/ and institutions and are being supported

- Development of local area network on government buildings and other public institutions

- E-mail directory has been developed for all public servants

- Government Common Core Network (GCCN) - Government wide area network is underway.

However, Desai, et al. (2002), in their assessment of various countries in respect of their Technology Achievement Index (TAI) ${ }^{8}$, rank Kenya as a marginalised country with a TAI of 0.129 (see table 1 below). Its technology diffusion and skill building are insufficiently developed with large parts of the population not having benefited from technology (Government of Kenya 2007).

\footnotetext{
${ }^{8}$ The TAI is designed to give a snapshot of the diffusion of bio- and information technology within the nations of the world, which nations are investing most in these technologies and how countries promote technological advancement through education. It is rendered by a simple average of the four dimension indices highlighted below where each dimension is scored between 0-1 with a higher number indicating higher technology achievement.
} 
The TAI captures data on the performance of countries in creating and diffusing technology and building a human skills base. The index measures eight indicators in the following dimensions:

- Creation of technology, as measured by the number of patents granted to residents per capita and by receipts of royalties and license fees from abroad per capita

- Diffusion of new innovations, as measured by the number of Internet hosts per capita and the share of high- and medium-technology exports in total goods exports

- Diffusion of old innovations, as measured by telephones (mainline and cellular) per capita and electricity consumption per capita

- Human skill, as measured by mean years of school in the population age 15 and above and the gross tertiary science enrolment ratio (Desai, Fukuda-Parr et al. 2002, p.98).

Table A-3.1: Kenya’s Technology Achievement Index Status, 1995-2000

\begin{tabular}{|c|c|c|c|}
\hline Country & TAI rank & TAI & HDI rank \\
\hline South Korea & 5 & 0.666 & 28 \\
\hline Malaysia & 30 & 0.396 & 59 \\
\hline South Africa & 39 & 0.340 & 119 \\
\hline Thailand & 40 & 0.337 & 76 \\
\hline China & 45 & 0.299 & 94 \\
\hline India & 63 & 0.201 & 127 \\
\hline Kenya & 68 & 0.129 & 148 \\
\hline
\end{tabular}

Source: Desai, et al. (2002)

The TAI reflects a country's level of technological progress and its capacity to participate in the networked economy. It is a measure of how well a nation is participating in creating and using technology (Desai, Fukuda-Parr et al. 2002; Government of Kenya 2007).

The ArCo Technology Index, which is built on three main dimensions of technological capabilities seen as playing a key role in the making of the technological capabilities of a nation - the creation of technology; the diffusion of technology and the development of human skills — ranks Kenya under the list of marginalised nations (Archibugi and Coco 2004; Archibugi and Coco 2005; Government of Kenya 2007). Under the ArCo Technology Index, the various countries are ranked as leaders (from 1-25 rankings), potential leaders (from 2650), latecomers (from 51-111) or marginalised (from 112 -162) (see table 2 below). 
Table A-3.2: Kenya’s ArCo Technology Index, 2004

\begin{tabular}{|l|l|l|l|l|l|}
\hline & $\begin{array}{l}\text { Actual } \\
\text { ranking }\end{array}$ & $\begin{array}{l}\text { Actual } \\
\text { ArCo } \\
\text { Index }\end{array}$ & $\begin{array}{l}\text { Past ArCo } \\
\text { Index }\end{array}$ & $\begin{array}{l}\text { Past } \\
\text { ranking }\end{array}$ & $\begin{array}{l}\text { Growth rate from the } \\
\text { last decade (\%) }\end{array}$ \\
\hline Korea, South & 20 & 0.601 & 0.414 & 31 & 45.20 \\
\hline South Africa & 56 & 0.372 & 0.336 & 58 & 11.00 \\
\hline Malaysia & 58 & 0.369 & 0.295 & 69 & 25.20 \\
\hline Thailand & 68 & 0.342 & 0.278 & 80 & 23.20 \\
\hline China & 86 & 0.306 & 0.227 & 97 & 34.60 \\
\hline Mauritius & 93 & 0.284 & 0.231 & 95 & 23.30 \\
\hline Botswana & 103 & 0.254 & 0.189 & 109 & 34.60 \\
\hline India & 111 & 0.225 & 0.169 & 116 & 32.80 \\
\hline Kenya & 116 & 0.204 & 0.177 & 114 & 15.20 \\
\hline Nigeria & 128 & 0.142 & 0.115 & 127 & 23.50 \\
\hline
\end{tabular}

To harness the potential of ICT for economic growth and poverty reduction, the Kenyan government has developed the following legal and regulatory frameworks:

- $\quad$ The National ICT Strategy 2004

- Information and Communications Technology Sector Policy Guidelines 2006 under the Kenya Gazette No. 2431 of 31st March, 2006.

Currently, the government is in the process of developing a national Science, Technology and Innovation (STI) policy based on four guiding principles: infrastructure development, human resource development, stakeholder participation and appropriate policy and regulatory framework (Government of Kenya 2007). The STI system is seen as central for the empowerment of all Kenyans as they seek to achieve social, political, economic and environmental goals through development of innovative ideas, products, institutional arrangements and processes in the context of global economic competitiveness, sustainable development and equity considerations. The STI is viewed as a facilitator to economic growth and elimination of absolute poverty; hence, a major key to development (Government of Kenya 2007). ICT is a core sector of the national STI policy, and the development of a supporting ICT infrastructure, services, local content and research and development in ICT are a national key priority. 


\section{A - 4: Summary of selected empirical studies on diffusion of IT- related innovations}

\begin{tabular}{|c|c|c|c|}
\hline Reference & $\begin{array}{l}\text { Research } \\
\text { Method }\end{array}$ & $\begin{array}{l}\text { IT Innovation } \\
\text { studied }\end{array}$ & Factors explored \\
\hline $\begin{array}{l}\text { Rogers (Rogers 1986; } \\
\text { Rogers 1993; Rogers } \\
\text { 2003) } \\
\text { Rogers \& Shoemaker } \\
(1971)\end{array}$ & All & General & $\begin{array}{c}5 \text { innovation characteristics: } \\
\text { - } \text { Compatibility } \\
\text { - } \\
\text { - } \\
\text { - } \\
\text { - } \\
\text { Trialablability } \\
\text { Observability }\end{array}$ \\
\hline $\begin{array}{l}\text { Tornatzky \& } \quad \text { Klein } \\
\text { (1982) }\end{array}$ & $\begin{array}{l}\text { Review of } \\
\text { research } \\
\text { works }\end{array}$ & General & $\begin{array}{l}5 \text { more major innovation characteristics: } \\
\text { - Cost } \\
\text { - Communicability } \\
\text { - Divisibility } \\
\text { - } \text { Profitability } \\
\text { - } \\
\text { Social approval }\end{array}$ \\
\hline Zmud (1982) & Survey & IT & Identifying centralisation and formalisation as factors \\
\hline Zmud (1984) & $\ldots$ & $\begin{array}{l}\text { Modern S/W } \\
\text { practices }\end{array}$ & Size and professionalism influence initiation \\
\hline Huff \& Munro (1985) & $\ldots$ & IT & Importance of initiation and adoption strategies \\
\hline $\begin{array}{l}\text { Chakrabarty, Feinman, } \\
\text { \& Fuentevilla (1983) }\end{array}$ & Survey & General & Influence of sources and channels \\
\hline Zmud,(1983) & Survey & Software groups & Influence of external channels \\
\hline Kwon \& Zmud (1987) & $\ldots$ & IT in general & $\begin{array}{ll}\text { Identified five types of factor models: } \\
\text { - } \\
\text { - } \\
\text { - } \\
\text { - } & \text { Tasnovation or technical } \\
\text { - } & \text { Environment } \\
\text { Individual }\end{array}$ \\
\hline $\begin{array}{ll}\text { Brancheau } & \& \\
\text { Wetherbe (1990) } & \end{array}$ & Survey & Spread sheet & $\begin{array}{l}\text { Early adopters: } \\
\text { Young, highly educated, more tuned to mass media } \\
\text { and involved in interpersonal communication }\end{array}$ \\
\hline $\begin{array}{lll}\text { Cooper } & \& & \text { Zmud } \\
(1990)\end{array}$ & $\begin{array}{l}\text { Telephone } \\
\text { survey }\end{array}$ & MRP systems & $\begin{array}{l}\text { The interaction of Managerial task with IT influence } \\
\text { adoption at various stages }\end{array}$ \\
\hline $\begin{array}{l}\text { Nilakanta \& Scamell } \\
(1990)\end{array}$ & Survey & Dbase systems & $\begin{array}{l}\text { Stage model. Examines effects of information } \\
\text { sources and communication channels }\end{array}$ \\
\hline $\begin{array}{l}\text { Moore \& Benbasat, } \\
(1991)\end{array}$ & Survey & $\ldots$ & $\begin{array}{l}\text { Instrument development based on Rogers' innovation } \\
\text { criteria }\end{array}$ \\
\hline $\begin{array}{l}\text { Grover \& } \quad \text { Goslar, } \\
\text { (1993) }\end{array}$ & $\begin{array}{l}\text { IS executive } \\
\text { survey }\end{array}$ & 15 Telecom products & $\begin{array}{l}\text { Environmental uncertainty, decentralisation of } \\
\text { decision-making as factors }\end{array}$ \\
\hline Davis (1989) & $\begin{array}{l}\text { Survey }+ \\
\text { Case study }\end{array}$ & IT & $\begin{array}{l}\text { Usefulness, Ease of use, User acceptance as factors } \\
\text { of IT adoption }\end{array}$ \\
\hline Swanson (1994) & Theory & IT in general & $\begin{array}{l}\text { Professional organisational sub units, institutional } \\
\text { arrangements }\end{array}$ \\
\hline $\begin{array}{l}\text { Premkumar, } \\
\text { Ramamurthy, } \quad \& \\
\text { Nilakanta (1994) }\end{array}$ & $\begin{array}{l}\text { Empirical } \\
\text { and survey }\end{array}$ & EDI & $\begin{array}{l}\text { Identifies relationship between innovation } \\
\text { characteristics and stages of diffusion }\end{array}$ \\
\hline $\begin{array}{l}\text { Prescott \& Conger, } \\
(1995)\end{array}$ & Review & IT in general & $\begin{array}{l}\text { A survey of DOI research: } \\
\text { Locus of impact }\end{array}$ \\
\hline $\begin{array}{l}\text { Premkumar \& Potter } \\
(1995)\end{array}$ & $\begin{array}{l}\text { Empirical } \\
\text { and survey }\end{array}$ & CASE & $\begin{array}{l}\text { Identifies various organisational and technological } \\
\text { characteristics in CASE adoption }\end{array}$ \\
\hline $\begin{array}{l}\text { O'Callaghan, } \\
\text { Kaufmann, } \quad \& \\
\text { Konsynski (1992) }\end{array}$ & $\ldots$ & EDI & $\begin{array}{l}\text { Innovation factors: relative advantage and } \\
\text { compatibility }\end{array}$ \\
\hline Iivari (1995) & Survey & CASE & $\begin{array}{l}\text { Factors for non use; Relationship among innovation } \\
\text { parameters }\end{array}$ \\
\hline Iacovou, Benbasat, \& & Case study & EDI & Innovation factors: benefits, readiness, external \\
\hline
\end{tabular}




\begin{tabular}{|c|c|c|c|}
\hline Reference & $\begin{array}{l}\text { Research } \\
\text { Method }\end{array}$ & $\begin{array}{l}\text { IT Innovation } \\
\text { studied }\end{array}$ & Factors explored \\
\hline Dexter (1995) & & & factors \\
\hline $\begin{array}{l}\text { Hart and Saunders, } \\
\text { (1997) }\end{array}$ & Theory & EDI & The role of power and interpersonal trust \\
\hline $\begin{array}{l}\text { Dekimpe, Parker, \& } \\
\text { Sarvary (2000) }\end{array}$ & $\begin{array}{l}\text { Empirical } \\
\text { and Theory }\end{array}$ & $\begin{array}{l}\text { Digital } \\
\text { telecommunication } \\
\text { switches }\end{array}$ & $\begin{array}{l}\text { The role of GNP, ethnic heterogeneity, installed base } \\
\text { of old technology on adoption stages }\end{array}$ \\
\hline $\begin{array}{l}\text { Fichman \& Kemerer, } \\
\text { (1999) }\end{array}$ & Theory & IT & $\begin{array}{l}\text { Assimilation gap between acquisition and } \\
\text { development and its measure }\end{array}$ \\
\hline Gurbaxani (1990) & $\begin{array}{l}\text { Historical } \\
\text { time series } \\
\text { data }\end{array}$ & BITNET & Supports contagion hypothesis \\
\hline $\begin{array}{ll}\text { Gurbaxani } & \& \\
\text { Mendelson (1990) } & \end{array}$ & $\ldots$ & IT budget spending & Introduces new and extended diffusion models \\
\hline Abrahamson (1991) & $\ldots$ & General & $\begin{array}{l}\text { Anti-diffusion approach: Why certain technology } \\
\text { diffusion succeeds/fails. The role of social networks: } \\
\text { links and structure }\end{array}$ \\
\hline Rai et al. (1998) & $\begin{array}{l}\text { Historical } \\
\text { time-series } \\
\text { data }\end{array}$ & Internet & Challenges contagion hypothesis \\
\hline Mutula (2001) & $\begin{array}{l}\text { Literature } \\
\text { review }\end{array}$ & IT & $\begin{array}{l}\text { The IT environment in Kenya and implications for } \\
\text { public libraries }\end{array}$ \\
\hline (Mutula 2001) & $\begin{array}{l}\text { Literature } \\
\text { review }\end{array}$ & Internet & Internet access in East Africa \\
\hline $\begin{array}{l}\text { Ondari-Okemwa } \\
(2002)\end{array}$ & $\begin{array}{l}\text { Empirical } \\
\text { and case } \\
\text { study }\end{array}$ & AVU & $\begin{array}{l}\text { Challenges of harnessing virtual information } \\
\text { resources in Kenya: The case of the African virtual } \\
\text { university. }\end{array}$ \\
\hline Tetiwat (2003) & $\begin{array}{l}\text { Empirical } \\
\text { and survey }\end{array}$ & $\begin{array}{l}\text { Web-based } \\
\text { educational } \\
\text { technology }\end{array}$ & $\begin{array}{l}\text { A cross-cultural comparative study of factors } \\
\text { influencing the adoption and diffusion of web-based } \\
\text { educational technology in Thailand and New Zealand }\end{array}$ \\
\hline Tran (2006) & $\begin{array}{l}\text { Empirical } \\
\text { and survey }\end{array}$ & $\begin{array}{l}\text { Community } \\
\text { information networks }\end{array}$ & $\begin{array}{l}\text { Diffusion of community information networks in } \\
\text { public libraries in New Zealand }\end{array}$ \\
\hline Bagchi \& Udo (2007) & $\begin{array}{l}\text { Empirical } \\
\text { and Theory }\end{array}$ & ICT & $\begin{array}{l}\text { Empirically testing factors that drive ICT adoption in } \\
\text { Africa and OECD }\end{array}$ \\
\hline $\begin{array}{l}\text { Odero-Musakali } \quad \& \\
\text { Mutula (2007) }\end{array}$ & $\begin{array}{l}\text { Literature } \\
\text { survey and } \\
\text { authors, } \\
\text { personal }\end{array}$ & Internet & $\begin{array}{l}\text { Internet adoption and assimilation in Kenyan } \\
\text { university libraries }\end{array}$ \\
\hline $\begin{array}{l}\text { Bakkabulindi, et al. } \\
(2008)\end{array}$ & $\begin{array}{l}\text { Empirical } \\
\text { and survey }\end{array}$ & ICT & $\begin{array}{l}\text { Organisational characteristics as correlates of ICT } \\
\text { adoption in Makerere university. }\end{array}$ \\
\hline Keengwe, et al. (2009) & $\begin{array}{l}\text { Empirical } \\
\text { and } \\
\text { qualitative }\end{array}$ & ICT & $\begin{array}{l}\text { Factors affecting ICT adoption and implications for } \\
\text { faculty training and technology leadership }\end{array}$ \\
\hline $\begin{array}{l}\text { Rogers, E. M., } \\
\text { Takegami, S. \& Yin, J. } \\
\text { (2001) }\end{array}$ & & Technology & Lessons learnt about technology transfer \\
\hline $\begin{array}{l}\text { Van de Ven, A. \& } \\
\text { Rogers, E. (1988) }\end{array}$ & & Innovations & Innovations and organizations \\
\hline $\begin{array}{l}\text { Damanpour, F. \& } \\
\text { Schneider, M. } \\
\text { (2009) }\end{array}$ & & Innovations & $\begin{array}{l}\text { Characteristics of innovation and innovation adoption } \\
\text { in public organisations: Assessing the role of } \\
\text { managers }\end{array}$ \\
\hline $\begin{array}{l}\text { Damanpour, F. \& \& } \\
\text { Schneider, M. (2006) }\end{array}$ & & Innovation & $\begin{array}{l}\text { Phases of adoption of innovation in organisations: } \\
\text { Effects of environment, organization, and top } \\
\text { managers }\end{array}$ \\
\hline
\end{tabular}




\section{A - 5: Summary of empirical studies on the cultural factor in IT/ICT diffusion}

\begin{tabular}{|c|c|}
\hline Reference & Focus of the study \\
\hline Hofstede $(1980 ; 1980)$ & $\begin{array}{cl}\text { Identifies four Cultural dimensions: } \\
\text { - } & \text { Power distance } \\
\text { - } & \text { Uncertainty avoidance } \\
\text { - } & \text { Individualism/collectivism } \\
\text { - } & \text { Masculinity/femininity }\end{array}$ \\
\hline $\begin{array}{l}\text { Hofstede \& Bond } \\
(1992)\end{array}$ & $\begin{array}{l}\text { Identifies one additional dimension: } \\
\text { • Confucian dynamism (Long/short term orientation) }\end{array}$ \\
\hline $\begin{array}{lr}\text { Inglehart } & (1995) ; \\
\text { Inglehart } & \& \\
\text { Baker(2000); } & \text { Inglehart, } \\
\text { Basanez, \& } & \text { Moreno } \\
(1998) & \end{array}$ & $\begin{array}{ll}\text { Identifies new dimensions: } \\
\text { • } & \text { Traditional/secular-rational } \\
\text { - } & \text { Survival/self-expression }\end{array}$ \\
\hline $\begin{array}{l}\text { Schwarz \& Bilsky } \\
\text { (1990); Schwarz \& } \\
\text { Sagiv (1995) }\end{array}$ & $\begin{array}{ll}\text { - } & \text { Identifies new dimensions: } \\
\text { - } & \text { Self-direction } \\
\text { - } & \text { Hedonism } \\
\text { - } & \text { Tradition } \\
\text { - } & \text { Power } \\
\text { - } & \text { Stimulation } \\
\text { - } & \text { Universalism } \\
\text { - } & \text { Benevolence } \\
\text { - } & \text { Conformity } \\
\text { - } & \text { Security } \\
\text { - } & \text { Achievement }\end{array}$ \\
\hline $\begin{array}{l}\text { Trompenaars \& \& } \\
\text { Hamden-Turner (1993) }\end{array}$ & $\begin{array}{ll}\text { Identifies new dimensions: } \\
\text { - Universalism/particularism } \\
\text { - } \quad \text { Individualism/communitarianism } \\
\text { - } \quad \text { Neutral/emotional } \\
\text { - } \\
\text { - } \\
\text { - Achecific/diffuse } \\
\text { Attitude to time and environment }\end{array}$ \\
\hline $\begin{array}{l}\text { Smith, Dugan, \& } \\
\text { Trompenaars (1996) }\end{array}$ & Earlier versions of Trompenaars dimensions \\
\hline Hoppe (1990) & Validates Hofstede’s work \\
\hline Sondergaard (1994) & Survey of works based on Hofstede’s dimensions \\
\hline Kedia \& Bhagat (1988) & Theorises impact of culture on technology transfer \\
\hline Kogut \& Singh (1988) & Develops measures of cultural distance, based o Hofstede’s dimensions \\
\hline Mejias (1995) & $\begin{array}{l}\text { Applies Hofstede's dimensions in DSS systems diffusion in Mexico and the } \\
\text { US }\end{array}$ \\
\hline $\begin{array}{l}\text { Milberg, Burke, Smith, } \\
\text { \& Kallman (1995) }\end{array}$ & Applies Hofstede’s dimensions in DSS systems \\
\hline $\begin{array}{l}\text { Herbig \& Muller } \\
\text { (1992) }\end{array}$ & Explores relationship between technology and culture \\
\hline Baskerville (1995) & Structural entropy in technology transfer \\
\hline Straub (1994) & $\begin{array}{l}\text { Explores role of culture on e-mail and FAX diffusion between Japan and the } \\
\text { US }\end{array}$ \\
\hline $\begin{array}{l}\text { Straub, Hill, Loch, \& } \\
\text { El-Sheshai (1998) }\end{array}$ & Study Arab culture and information technology transfer \\
\hline Harvey (1997) & $\begin{array}{l}\text { An evaluation of Hofstede's national cultural framework to establish } \\
\text { national cultural difference in theory and practice }\end{array}$ \\
\hline Bunker \& Dean (1998) & $\begin{array}{l}\text { Study the cultural context of creation and use of information technology and } \\
\text { systems as tools }\end{array}$ \\
\hline El-Shinnawy \& Vinze & Explore choice-shifts in group settings in relation to technology, culture and \\
\hline
\end{tabular}




\begin{tabular}{|c|c|}
\hline Reference & Focus of the study \\
\hline$(1997)$ & persuasiveness \\
\hline Evers \& Day (1997) & Explore the role of culture in interface acceptance \\
\hline Hill, et al., (1998) & $\begin{array}{l}\text { A qualitative assessment of Arab culture and information technology } \\
\text { transfer }\end{array}$ \\
\hline Hasan \& Ditsa (1999) & An interpretive study to explore the impact of culture on the adoption of IT \\
\hline Cooper (1994) & Explores the inertial impact of culture on IT implementation \\
\hline $\begin{array}{l}\text { Segars, Grover, \& } \\
\text { Durand (1994) }\end{array}$ & $\begin{array}{l}\text { Explore cultural diversity in organisational practice, information resource } \\
\text { deployment and system success among French, Korean and US firms }\end{array}$ \\
\hline $\begin{array}{l}\text { Goodman } \\
\begin{array}{l}\text { Goodman } \\
(1992)\end{array}\end{array} \quad \& \quad$ Green & Conjectures role of culture on IT diffusion across nations \\
\hline $\begin{array}{llr}\begin{array}{l}\text { Marcus } \\
(2000)\end{array} & \& & \text { Gould } \\
\end{array}$ & Explore cultural dimensions in global web user-interface design \\
\hline $\begin{array}{l}\text { Png, Tan, \& Wee } \\
(2001)\end{array}$ & $\begin{array}{l}\text { Address dimensions of national culture in corporate adoption of IT } \\
\text { infrastructure }\end{array}$ \\
\hline Bagchi (2001) & $\begin{array}{l}\text { Studies the impact of national culture and other national-level indicators on } \\
\text { information technology (IT) diffusion }\end{array}$ \\
\hline Tetiwat (2003) & $\begin{array}{l}\text { Applies Hofstede's dimensions in a comparative cross-cultural study of } \\
\text { factors influencing the adoption and diffusion of web-based educational } \\
\text { technology in New Zealand and Thailand }\end{array}$ \\
\hline Kaye \& Little (2004) & $\begin{array}{l}\text { Explore cultural conflicts that lead to dysfunctional development pathways } \\
\text { of information and communication technology }\end{array}$ \\
\hline Hill, et al., (1998) & $\begin{array}{l}\text { A qualitative assessment of Arab culture and information technology } \\
\text { transfer }\end{array}$ \\
\hline $\begin{array}{l}\text { Huo, Y.P. \& Randall, } \\
\text { D.M. (1991) }\end{array}$ & $\begin{array}{l}\text { Explore subcultural differences in Hofstede's value survey among the case } \\
\text { of the Chinese }\end{array}$ \\
\hline McCoy, S. (2003) & $\begin{array}{l}\text { A criticism of Hofstede national culture perspective - the need to factor } \\
\text { individual level measures in integrating national culture }\end{array}$ \\
\hline McSweeney, B. (2002) & $\begin{array}{l}\text { A criticism of Hofstede's model of national cultural differences and their } \\
\text { consequences: A triumph of faith - a failure of analysis }\end{array}$ \\
\hline $\begin{array}{l}\text { Myers, M. \& Tan, F. } \\
(2002)\end{array}$ & $\begin{array}{l}\text { A criticism of Hofstede's model - Beyond models of national culture in } \\
\text { information systems research }\end{array}$ \\
\hline $\begin{array}{l}\text { Nath, R. \& Murphy, N. } \\
(2004)\end{array}$ & A study of the relationship between Internet diffusion and culture \\
\hline Tayeb, M. (1994) & Methodology in studies on organisations and national culture \\
\hline $\begin{array}{l}\text { Weisinger, J.Y. } \quad \& \\
\text { Trauth, E.M. (2003) }\end{array}$ & The Importance of Situating Culture in Cross-Cultural IT Management \\
\hline
\end{tabular}

